\title{
Topologias de grupo enumeravelmente compactas: \\ MA, forcing e ultrafiltros seletivos
}

\author{
Jury Fabiana Castiblanco Quiroga
}

\author{
DISSERTAÇÃO APRESENTADA \\ AO \\ Instituto DE MATEMÁticA E EstATÍSTICA \\ DA \\ UNiversidade de SÃo PAUlo \\ PARA \\ OBTENÇÃO DO TÍTULO \\ $\mathrm{DE}$ \\ Mestre em CiênCIAS
}

Programa: Matemática

Orientador: Prof. Dr. Artur Hideyuki Tomita

Durante o desenvolvimento deste trabalho a autora recebeu apoio financeiro da FAPESP

Processo No. 2009/03768-1

São Paulo, novembro de 2011 



\title{
Topologias de grupo enumeravelmente compactas: MA, forcing e ultrafiltros seletivos
}

\author{
Esta dissertação contém as correções e alterações \\ sugeridas pela Comissão Julgadora durante a defesa \\ realizada por Jury Fabiana Castiblanco Quiroga em 07/11/2011. \\ O original encontra-se disponível no Instituto de \\ Matemática e Estatística da Universidade de São Paulo.
}

Comisão Julgadora:

- Prof. Dr. Artur Hideyuki Tomita (orientador) - IME-USP

- Profa. Dra. Christina Brech - IME-USP

- Profa. Dra. Irene Castro Pereira - UFPA 

Aos meus pais, Gladys e Hugo.

Não há um só dia em que vocês não estejam no meu $\varnothing$ 



\section{Agradecimentos}

Em primeiro lugar agradeço aos meus pais, Gladys e Hugo, pelo enorme apoio, pelo imenso amor e dedicação que mostraram fazendo inúmeras renúncias para que os meus sonhos se tornassem realidades, por me ensinar a encarar o mundo de coração aberto, com alegria e coragem. Meu sentimento de gratidão a eles é tão grande que resulta impossível de descrever em umas poucas linhas.

Aos meus afetuosos avós, Gladys e Luis, aos meus tios, Mercedes e Armando e às minhas irmãs, Julieth e Fer, que com amor, estão sempre ao meu lado.

Obrigada Pedro, por teu inestimável carinho, paciência, dedicação, por me acompanhar sempre -apesar da distância- e me infundir ânimo com uma palavra meiga nas circunstâncias mais difíceis.

Aos meus amores Negra, Pacho, Estrellita, Flaco, Bambi, Fríjol e Polly por não me esquecer e por sua infinita ternura; essas beldades sabem o que na alma pode representar um sorriso :)

À Nubia, pela sua generosidade, incrível amizade e apropriados conselhos antes, durante e, com certeza, depois deste passo na minha vida.

Ao Rafael, por me oferecer um lar quando mais eu precisei, e a Dona Célia, pelo carinho de mãe que sempre me brindou.

Também sou muito grata ao Cleber, um amigo afável, sincero e incondicional. Aos outros colegas da sala 155B, Jana, Berna, Gustavo, Silvana, Henry e os Brunos, pelas risadas e bons momentos compartilhados durante estes anos. Agradeço também aos colegas de sala mais recentes, Gabriel e André, por ouvirem minhas aflições e alegrias em diversas ocasiões. 
A Ale Aldarete, Deissy e Diana pela companhia, abraços e colaboração.

Aos meus grandes amigos em Bogotá, Jairo, Sebas, Lauri, Jennifer, Jeffer e Javier pelo apoio moral e os carinhos recebidos apesar da distância.

Ao César, quem me acolheu calorosamente em Bonn durante alguns dias prévios ao Young Set Theory Workshop 2011, onde finalmente compreendi o maravilhoso da técnica do forcing. Agradeço aos Profs. Ernesto Acosta, Lucimar Nova e Leonardo Rendón pelos inapreciáveis ensinamentos que me dispensaram durante a graduação na Universidad Nacional de Colombia.

Minha gratidão para o Prof. Dr. Artur Tomita, meu orientador, pelo apoio, os conselhos, a paciência com minhas faltas e a compreensão com a fragilidade de minha saúde.

Agradeço também às professoras integrantes da banca examinadora, Irene Castro e Christina Brech, pelo tempo que dedicaram a ler este texto e pelas valiosas sugestões e apontamentos que, sem dúvida, o melhoraram.

Por fim, expresso de maneira muito especial minha gratidão à Fundação de Amparo à Pesquisa do Estado de São Paulo (FAPESP) pelo apoio financeiro concedido gentilmente, sem o qual, este trabalho não teria sido possível. 
“... das Wesen der Mathematik liegtgerade in ihrer Freiheit”.

G. CANTOR 



\section{Resumo}

\section{Topologias de grupo enumeravelmente compactas: MA, forcing e ultrafiltros seletivos}

É bem conhecido o fato de que todo grupo compacto tem sequências não triviais convergentes. A existência de grupos enumeravelmente compactos sem sequências não triviais convergentes, foi provada usando axiomas adicionais à axiomática usual ZFC: A. Hajnal e I. Juhász [39] sob CH, E. K. van Douwen [26] sob MA, A. H. Tomita sob MA o-centrada e R.E. Madariaga-Garcia e A. H. Tomita [50] usando ultrafiltros seletivos.

Neste trabalho, estudaremos algumas construções recentes relacionadas com as citadas acima, usando o Axioma de Martin, ultrafiltros seletivos e forcing. Essas construções estão relacionadas com algumas questões indicadas por A.D. Wallace, E. van Douwen, M. Tkačenko, D. Dikranjan e D. Shakhmatov.

Palavras-chave: Forcing, compacidade enumerável, grupo topológico, Axioma de Martin, ultrafiltro seletivo. 



\section{Abstract}

Countably compact group topologies:

\section{MA, forcing and selective ultrafilters}

It is well known that every compact group has non-trivial convergent sequences. The existence of countably compact groups without non-trivial convergent sequences was proved using extra set-theoretical assumptions: A. Hájnal and I. Juhász [39] under CH, E. K. van Douwen [26] under MA, A. H. Tomita under $\mathrm{MA}_{\text {o-centered }}$ and R.E. Madariaga-Garcia and A. H. Tomita [50] using a selective ultrafilter.

In this work, we study some recent constructions related to the ones given above using Martin Axiom, selective ultrafilters and forcing, related to questions raised by A.D. Wallace, E. van Douwen, M. Tkačenko, D. Dikranjan and D. Shakhmatov.

Keywords: Forcing, countable compactness, topological group, Martin Axiom, selective ultrafilter. 



\section{Sumário}

Lista de símbolos $\quad$ xiii

Introdução 1

1 Conceitos e resultados preliminares $\quad 5$

1.1 Teoria dos Conjuntos $\ldots \ldots \ldots \ldots \ldots \ldots$

1.2 Topologia . . . . . . . . . . . . . . . . . . . . . . 18

1.3 Grupos topológicos e estruturas relacionadas . . . . . . . . . . . . . . 22

2 Grupos de van Douwen e MA 27

2.1 Primeira construção de E. van Douwen a partir de MA f . . . . . . . . . . 29

2.2 Grupos abelianos livres e compacidade enumerável . . . . . . . . . . . . 35

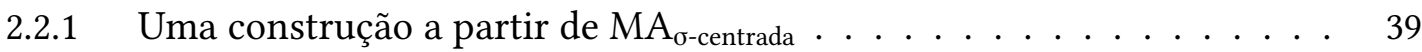

2.2.2 Uma construção a partir de $\mathrm{MA}_{\text {enumerável }} \ldots \ldots$. . . . . . . . . . . 47

2.2.3 Uma topologia de grupo e.c. sobre $\mathrm{A}\left(2^{c}\right)$ via extensões genéricas _ . . 53

3 Topologias de grupo e ultrafiltros seletivos $\quad 61$

3.1 Espaços $p$-compactos . . . . . . . . . . . . . . . . . 61

$3.2 p$-compacidade e ultrafiltros seletivos $\ldots \ldots \ldots \ldots \ldots$

3.2.1 Ultraprodutos e $p$-compacidade . . . . . . . . . . . . . . . 67

3.2.2 $\omega^{*}$ não é um conjunto dirigido na ordem $\leq_{\mathrm{CG}} \ldots \ldots \ldots \ldots$. . . . . . 74

3.3 Grupos de van Douwen livres e ultrafiltros seletivos . . . . . . . . . . . . . . . 80

3.3.1 Codificando as seqüências em um grupo abeliano livre . . . . . . . . . 81

3.3.2 A construção dos homomorfismos . . . . . . . . . . . . . . 82 
3.3.3 Existência de ultrafiltros seletivos e MA: Alguns resultados de consis-

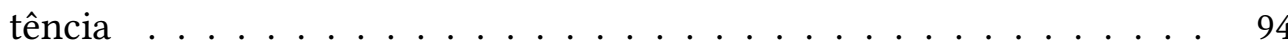

$4 \quad$ Invariantes cardinais em grupos enumeravelmente compactos 97

4.1 Peso e cardinalidade de grupos pseudocompactos f . . . . . . . . . . 98

4.2 A questão de van Douwen . . . . . . . . . . . . . . . . . . 106

4.2.1 Um grupo enumeravelmente compacto de cardinalidade $\boldsymbol{\aleph}_{\omega} \ldots \ldots \ldots$

4.2.2 Um grupo enumeravelmente compacto de peso $\aleph_{\omega} \ldots \ldots$. . . . . . . 113

5 Problemas relativos a topologias de grupo enumeravelmente compactas $\quad 119$

5.1 O problema de Wallace . . . . . . . . . . . . . . . . . . . . . . . . . 119

5.2 Uma questão de Dikranjan e Shakhmatov . . . . . . . . . . . . . . . . . . . . . 124

5.3 Sobre topologias de grupo independentes . . . . . . . . . . . . . . . 125

$\begin{array}{ll}\text { Referências Bibliográficas } & 145\end{array}$

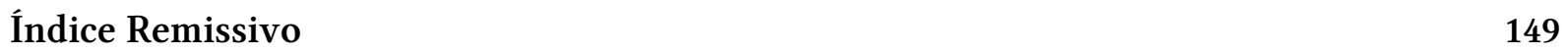




\section{Lista de símbolos}

$\begin{array}{ll}\mathbb{N} & \text { o conjunto dos números naturais } \\ \mathbb{R} & \text { o conjunto dos números reais } \\ \mathbb{T} & \text { o conjunto dos números complexos de norma } 1 \\ \boldsymbol{\aleph}_{0} & \text { o cardinal enumerável infinito } \\ \boldsymbol{\aleph}_{1} & \text { o primeiro cardinal não enumerável } \\ \boldsymbol{\aleph}_{n} & \text { o } n \text {-ésimo cardinal não enumerável, } n \geq 1 \\ \omega & \text { o primeiro ordinal enumerável infinito } \\ \omega_{1} & \text { o primeiro ordinal não enumerável } \\ \omega_{n} & \text { o primeiro ordinal de cardinalidade } \aleph_{n}, n \geq 1 \\ \mathcal{c} & \text { o cardinal do contínuo } \\ \omega^{*} & \text { o conjunto dos ultrafiltros livres sobre } \omega \\ \text { cf( } \alpha) & \text { a cofinalidade de } \alpha \\ A^{B} & \text { o conjunto das funções de } A \text { em } B \\ \text { Fn }(A, B) & \text { o conjunto das funções finitas de } A \text { em } B \\ f \Gamma_{S} & \text { a função } f \text { restrita ao conjunto } S \\ \chi_{S} & \text { a função caraterística do conjunto } S \\ |A| & \text { a cardinalidade do conjunto } A \\ {[A]^{\kappa}} & \text { o conjunto dos subconjuntos de } A \text { de cardinalidade } \kappa \\ \mathcal{A} \vDash \Gamma & \mathcal{A} \text { é um modelo de } \Gamma \\ p \Vdash \phi & \text { a condição } p \text { força a afirmação } \phi \\ \mathbb{S} & \text { o forcing de Sacks } \\ \mathbb{S}_{\omega}^{\kappa} & \text { o forcing side-by-side de Sacks de comprimento } \kappa \\ w(X) & \text { o peso do espaço } X \\ d(X) & \text { a densidade do espaço } X \\ c(X) & \text { a celularidade do espaço } X \\ \chi(X) & \text { o caráter do espaço } X \\ & \end{array}$





\section{Introdução}

"In re mathematica ars proponendi quaestionem pluris facienda est quam solvendi”.

G. CANTOR

Se $X$ é um espaço topológico de Hausdorff e $\kappa$ é um cardinal tal que $\kappa<|X|$, dizemos que $X$ omite $\kappa$ se não possui subconjuntos fechados de cardinalidade $\kappa$. De acordo com a cardinalidade do espaço, existem espaços compactos que omitem $\omega$, mas se $|X|=\mathfrak{c}$, a questão não poder ser decidida na axiomática ZFC; de fato, Hajnal e Juhász [38] mostraram que sob a hipótese do contínuo $(\mathrm{CH})$ nenhum espaço compacto de cardinalidade c pode omitir $\omega$, enquanto que Fedorčuk [31] mostrou que a existência de um espaço compacto de cardinalidade c sem sequências não triviais convergentes é consistente com ZFC.

Contudo, se considerarmos uma estrutura topológica junto com uma estrutura algébrica, a situação se torna diferente. De maneira independente, Ivanovskii (1958) e Kuz'minov (1959) mostraram que todo grupo compacto é diádico, isto é imagem por uma função contínua de um cubo de Cantor generalizado, motivo pelo qual nenhum deles pode omitir $\omega$. Assim, resulta natural perguntar pela omissão de $\omega$ na classe dos grupos topológicos que satisfazem propriedades mais fracas porém próximas à compacidade, tais como a compacidade enumerável ou a pseudocompacidade.

Considerando grupos topológicos pseudocompactos, temos dois resultados principais. Em 1969 Sirota [58], respondendo a uma questão de Arkhangel'skǐ̌, construiu em ZFC um grupo pseudocompacto sem sequências não triviais convergentes de peso $\kappa$ para cada cardinal infinito $\kappa=\kappa^{\omega}$. Depois, em 1985, Malykhin e Shapiro [51] mostraram que a existência de um grupo pseudocompacto sem sequências não triviais convergentes de peso $\kappa<\kappa^{\omega}$ é independente de ZFC.

Apesar da compacidade enumerável constituir uma propriedade intermediária entre a compacidade e a pseudocompacidade, a existência de sequências não triviais convergentes 
nessas condições compreende uma questão bem mais sutil. De fato, até o presente momento essa questão não possui solução definitiva. O primeiro exemplo a este respeito, realizado sob CH, data de 1976 e deve-se a Hajnal e Juhász [39]; contudo, a construção desses autores não estava dirigida a construir um grupo enumeravelmente compacto sem sequências não triviais convergentes senão a proporcionar um exemplo de um grupo normal e separável que não fosse de Lindelöf. Mais tarde, diversos exemplos de grupos enumeravelmente compactos sem sequências não triviais convergentes foram construídos por van Douwen [26], Tkačenko, Tomita [66,67], Koszmider et al. [46] e Madariaga-Garcia e Tomita [50], sendo que todas essas construções foram obtidas assumindo asserções adicionais a ZFC ou por meio de extensões genéricas.

O propósito desta dissertação é estudar sistematicamente essas construções, focando nossa atenção nas que foram obtidas via forcing e ultrafiltros seletivos. Tentaremos mostrar a maneira em que a existência de um grupo enumeravelmente compacto sem sequências não triviais convergentes permite dar resposta a diversas questões que ao parecer só tem conexão com a compacidade enumerável.

São cinco capítulos os que compõem esta dissertação. O primeiro deles destina-se a estabelecer a notação utilizada no decorrer do texto além de expor alguns resultados e definições preliminares que serão necessários adiante. A primeira seção, é dedicada à teoria dos conjuntos introduzindo alguns resultados de combinatória, os conceitos de forcing e do Axioma de Martin (MA) e ainda, fazemos comentários relativos à consistência e independência em axiomáticas, relevantes ao longo do texto. A segunda seção, é dedicada a tópicos de topologia geral e a terceira trata de grupos topológicos.

No segundo capítulo, são apresentadas as construções de topologias de grupo enumeravelmente compactas sem sequências não triviais convergentes obtidas mediante o uso de MA, o axioma de Martin para ordens parciais $\sigma$-centradas $\left(\mathrm{MA}_{\sigma \text {-centrada }}\right)$ e o Axioma de Martin para ordens parciais enumeráveis $\left(\mathrm{MA}_{\text {enumerável }}\right)$. Na primeira seção, incluímos a construção de van Douwen [26] que, de acordo com a história do problema, é a pioneira usando MA. Na segunda seção, se estudam as construções relativas a topologias de grupo enumeravelmente compactas sobre grupos abelianos livres. Depois de apresentar definições e resultados básicos a respeito desse tipo de grupos, estudamos a construção devida a A.H. Tomita [66] de uma topologia de grupo enumeravelmente compacta definida sobre o grupo abeliano livre de cardinalidade

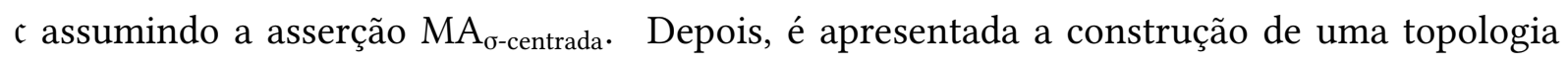
como a anteriormente referida mas, desta vez, assumindo a asserção $\mathrm{MA}_{\text {enumerável. }}$ A última 
parte do capítulo constitui o exemplo obtido via forcing por Koszmider et. al. [46] de uma topologia de grupo enumeravelmente compacta sobre o grupo abeliano livre de cardinalidade $2^{c}$.

O terceiro capítulo trata dos exemplos de topologias de grupo enumeravelmente compactas obtidas a partir de ultrafiltros seletivos. A primeira seção, é destinada a apresentar os conceitos e resultados básicos sobre ultrafiltros seletivos, ultraprodutos usando tal tipo de ultrafiltros e à relação destes últimos com a $p$-compacidade. A segunda seção, apresenta os resultados obtidos por Watson e Tomita [69] referentes à $p$-compacidade em grupos topológicos via ultrafiltros seletivos; nesta seção aparecem várias idéias das que vai fazer uso a construção da terceira e última seção do capítulo dedicada a descrever a maneira em que a existência de ultrafiltros seletivos permite construir topologias de grupo enumeravelmente compactas sobre grupos abelianos livres.

No quarto capítulo estudamos certas características cardinais dos grupos topológicos enumeravelmente compactos. Na primeira seção, são detalhados os resultados obtidos por van Douwen em [27] a respeito do peso e a cardinalidade de grupos topológicos pseudocompactos. Depois de estudar o comportamento desses invariantes em distintas aritméticas cardinais, no mesmo trabalho, van Douwen conjeturou que em todo grupo infinito enumeravelmente compacto $G$ se satisfaz $|G|^{\omega}=|G|$ ou, ao menos, $\operatorname{cf}(|G|)>\omega$, sendo esta afirmação consistente com ZFC. Contudo, acabou se revelando que a conjetura de van Douwen é independente de ZFC em virtude dos resultados obtidos por Tomita em [67] os quais abordamos na segunda seção deste capítulo.

Por fim, o último capítulo é dedicado a responder diversas questões fazendo uso das construções detalhadas nos capítulos precedentes; na primeira seção, descreve-se o problema de Wallace [71] e mostra-se que consistentemente existe um contraexemplo a dita questão. Na segunda seção, comentamos a resposta parcial à pergunta de Dikranjan e Shakhmatov pelas cardinalidades possíveis para os grupos abelianos livres que admitem uma topologia de grupo enumeravelmente compacta. A terceira seção trata-se sobre o trabalho de Tkačenko e Yasčenko [64] acerca de topologias de grupo independentes e a relação destas com a existência de topologias de grupo enumeravelmente compactas. 



\section{CAPÍTULO 1}

\section{Conceitos e resultados preliminares}

Neste capítulo, iremos fixar a notação utilizada no decorrer do texto além de fazer uma exposição sucinta de alguns tópicos necessários à compreensão do mesmo.

\subsection{Teoria dos Conjuntos}

Ao longo da dissertação trabalharemos baseando-nos na axiomática Zermelo-Fraenkel acrescida do Axioma da Escolha, abreviado por ZFC. Para os tópicos relacionados com teoria dos conjuntos, seguimos as referências [44, 47].

Começamos por reservar os símbolos $\mathbb{N}, \mathbb{Z}, \mathbb{Q}, \mathbb{R}$ e $\mathbb{C}$ para denotar, respectivamente, o conjunto dos números naturais, números inteiros, números racionais, números reais e números complexos.

A notação $B^{A}$ representa o conjunto de todas as funções $f: A \rightarrow B$. O domínio e a imagem de uma função $f \in B^{A}$, serão denotados por $\operatorname{dom}(f)$ e $\operatorname{im}(f)$ respectivamente. Se $S \subset A, f \uparrow_{S} \in B^{S}$ é a restrição de $f$ ao conjunto $S$. Se $S \subset A$ e $T \subset B, f[S]$ e $f^{-1}[T]$ denotam respectivamente a imagem de $S$ por $f$ e a pré-imagem de $T$ por $f$. Com freqüência, usaremos o símbolo $\operatorname{Fn}(A, B)$ para denotar o conjunto de todas as funções finitas de $A$ em $B$, isto é, $h \in \operatorname{Fn}(A, B)$ significa que $\operatorname{dom}(h)$ é um subconjunto finito de $A$ e $\operatorname{im}(h) \subset B$.

\section{Alguns resultados sobre ordinais e cardinais}

Definição 1.1.1. Um conjunto $M$ é transitivo se e somente se todo elemento de $M$ é também um subconjunto de $M$. 
Entendemos por ordinal um conjunto transitivo e bem ordenado pela relação $\in$. O símbolo $\mathbb{O N}$ representará a classe de todos os ordinais. Usaremos doravante letras gregas minúsculas (como $\alpha, \beta, \gamma, \ldots)$ para denotar elementos de $\mathbb{O N}$.

Se $\alpha \in \mathbb{O N}$ dizemos que $A \subset \alpha$ é ilimitado em $\alpha$ se $\sup A=\alpha$.

$\alpha \in \mathbb{O N}$ é dito um ordinal sucessor se existe $\beta \in \mathbb{O N}$ tal que $\alpha=\beta+1:=\beta \cup\{\beta\}$. Em caso contrário, dizemos que $\alpha$ é um ordinal limite. O menor ordinal limite infinito é o conjunto de todos os números naturais, denotado por $\omega$.

Se $X$ é um conjunto, uma função $f: \alpha \rightarrow X$ onde $\alpha \in \mathbb{O N}$ é dita seqüência de comprimento $\alpha$ em $X$. Freqüentemente, escreve-se $f=\left\langle f_{\xi}: \xi<\alpha\right\rangle$ ou $f=\left\{f_{\xi}: \xi<\alpha\right\}$ onde $f_{\xi}=f(\xi)$ para cada $\xi<\alpha$, identificando a $f$ com o conjunto im $(f)$. Se $s$ é uma seqüência de comprimento $\alpha$ num conjunto $X \ni x, s^{\sim} x$ denota a seqüência em $X$ de comprimento $\alpha+1$ dada por $s \cup\{(\alpha, x)\}$.

Definição 1.1.2. $\kappa \in \mathbb{O N}$ é dito um ordinal inicial se $\kappa=|\kappa|$, onde $|\kappa|$ é o menor $\alpha \in \mathbb{O N}$ tal que $\alpha$ é equipotente a $\kappa$.

Identificamos os ordinais iniciais exatamente com os números cardinais. Usaremos as letras gregas $\kappa, \lambda, \mu, \theta \ldots$ ou letras germânicas $\mathfrak{a}, \mathfrak{m}, \mathfrak{n}$.. para designar cardinais.

Se $X$ é um conjunto e $\kappa$ é um cardinal, fixamos a notação $[X]^{\kappa}=\{A \subseteq X:|A|=\kappa\} ;[X]^{<\kappa}$ $\mathrm{e}[X]^{\leq \kappa}$ são definidos analogamente.

Definição 1.1.3. Um cardinal $\kappa$ é dito sucessor se existe um cardinal $\lambda$ tal que $\kappa=\lambda^{+}$, onde $\lambda^{+}=\min \left\{\theta \leq 2^{\lambda}: \theta\right.$ é cardinal e $\left.\theta>\lambda\right\}$. Em caso contrário, dizemos que $\kappa$ é um cardinal limite.

Freqüentemente, os cardinais infinitos são chamados alephs; como cada cardinal possui um cardinal sucessor e para cada conjunto de cardinais $X$, sup $X$ é um cardinal, pode-se definir uma enumeração crescente de todos os alephs:

$$
\begin{aligned}
& \boldsymbol{\aleph}_{0}=\omega \\
& \boldsymbol{\aleph}_{\alpha+1}=\boldsymbol{\aleph}_{\alpha}^{+} \\
& \boldsymbol{\aleph}_{\alpha}=\sup \left\{\boldsymbol{\aleph}_{\xi}: \xi<\alpha\right\} \quad \text { se } \alpha \text { é um ordinal limite }
\end{aligned}
$$

Da enumeração anterior, temos que para cada $\alpha \in \mathbb{O N}, \boldsymbol{\aleph}_{\alpha}$ é cardinal sucessor ou limite conforme $\alpha$ seja ordinal sucessor ou limite. Denotaremos o tipo de ordem de $\boldsymbol{\aleph}_{\alpha}$ por $\omega_{\alpha}$.

Um dos mais célebres teoremas de Cantor diz que para cada conjunto $X$, vale que $|X|<$ $|\wp(X)|$. Em particular, se c representa a cardinalidade do conjunto dos números reais $\mathbb{R}$, 
temos que $\mathfrak{c}=2^{\aleph_{0}}>\aleph_{0}$. Em 1878, Cantor [14] conjeturou que todo subconjunto infinito de $\mathbb{R}$ é enumerável ou é equipotente a $\mathbb{R}$. Em ZFC, todo cardinal infinito é um aleph, logo, $\mathfrak{c} \geq \boldsymbol{\aleph}_{1}$; de onde a conjetura de Cantor resulta ser a asserção $2^{\aleph_{0}}=\aleph_{1}$, conhecida como a Hipótese do Contínuo $(\mathrm{CH})$.

Motivado pela afirmação de Cantor e pela relação $\aleph_{\alpha+1} \leq 2^{\aleph_{\alpha}}$ válida em ZFC para todo ordinal $\alpha$, F. Hausdorff [41] em 1908 enunciou a seguinte asserção $\forall \alpha \in \mathbb{O N}\left(2^{\aleph_{\alpha}}=\aleph_{\alpha+1}\right)$ conhecida como a Hipótese generalizada do Contínuo (GCH).

Convém apontar que as asserções $\mathrm{CH}$ e GCH não podem ser provadas nem refutadas no sistema axiomático ZFC, isto é, são independentes de ZFC.

Definição 1.1.4. Sejam $\alpha$ e $\beta \in \mathbb{O N}$. Se $f \in \beta^{\alpha}$, dizemos que $f$ é uma função cofinal se im $(f)$ é um conjunto ilimitado em $\beta$. A cofinalidade de um ordinal $\beta$ é o menor $\alpha \in \mathbb{O N}$ tal que existe uma função $f: \alpha \rightarrow \beta$ cofinal. Neste caso escreve-se $\operatorname{cf}(\beta)=\alpha$.

Um ordinal limite $\beta$ é dito regular se $\operatorname{cf}(\beta)=\beta$, e dito singular se $\operatorname{cf}(\beta)<\beta$. Deve apontarse que todo ordinal regular é um cardinal e que cada cardinal sucessor é um ordinal regular.

Definição 1.1.5. Um cardinal $\kappa$ é dito limite forte se para cada $\mu<\kappa, 2^{\mu}<\kappa$.

Definição 1.1.6. Um cardinal $\kappa>\omega$ é dito fracamente inacessível se é um cardinal regular e limite. Um cardinal fracamente inacessível $\kappa$ é dito inacessivel (ou fortemente inacessível) se também é limite forte.

Assumindo GCH as noções de cardinal fracamente inacessível e inacessível coincidem; por outro lado, não é possível provar em ZFC a existência de cardinais inacessíveis.

Por conveniência, incluímos a seguir um caso particular do denominado Teorema de König, importante fato que estabelece a desigualdade estrita mais geral conhecida (em ZFC) sobre cardinais infinitos.

Teorema 1.1.7. Se $\kappa$ é um cardinal infinito e $\operatorname{cf}(\kappa) \leq \lambda$, então $\kappa^{\lambda}>\kappa$.

Corolário 1.1.8 (Zermelo-König). Se $\lambda \geq \omega$, então $\operatorname{cf}\left(2^{\lambda}\right)>\lambda$.

\section{Uma nota sobre consistência e independência em axiomáticas}

Ao longo do texto irão ser construídos objetos matemáticos usando axiomas adicionais ao sistema ZFC; assim, resulta conveniente lembrar alguns fatos e definições provenientes da lógica matemática. A referência básica para tópicos mencionados neste parágrafo é [37]. 
Se $\mathcal{L}$ é uma linguagem de primeira ordem, uma $\mathcal{L}$-estrutura $\mathcal{A}$ consta de um conjunto não vazio $A$ (domínio de $\mathcal{A}$ ) e de relações, funções e constantes correspondentes aos símbolos da linguagem.

Definição 1.1.9. Se $\phi$ é uma fórmula em $\mathcal{L}$, dizemos que $\phi$ é uma sentença se não contem variáveis livres. Dada uma sentença $\phi$ e uma $\mathcal{L}$-estrutura $\mathcal{A}$, escrevemos $\mathcal{A} \vDash \phi$ se a sentença $\phi$ é verdadeira em $\mathcal{A}$.

Definição 1.1.10. Um modelo para um conjunto de sentenças $\Gamma$ numa linguagem $\mathcal{L}$, é uma $\mathcal{L}$-estrutura $\mathcal{A}$ tal que $\mathcal{A} \vDash \gamma$ para toda $\gamma \in \Gamma$. Neste caso, escrevemos $\mathcal{A} \vDash \Gamma$.

Se todo modelo de $\Gamma$ é também um modelo de $\phi$ (relação de conseqüência semântica) escrevemos $\Gamma \vDash \phi$. Baseando-nos num sistema dedutivo formal, dizemos que $\phi$ é um teorema de $\Gamma$ (relação de conseqüência sintática) se houver uma dedução formal nesse sistema de $\phi$ a partir de $\Gamma$. Escrevemos, nesse caso, $\Gamma \vdash \phi$.

Uma $\mathcal{L}$-teoria é um conjunto de sentenças $T$ em $\mathcal{L}$ fechado sob dedução. A $\mathcal{L}$-teoria $T$ é dita consistente se ela não pode deduzir contradições; de outra parte, $T$ é dita completa se é consistente e para toda sentença $\phi$ da linguagem, então $\phi \in T$ ou $\neg \phi \in T$. Se uma teoria consistente $T$ não é completa, então existe alguma sentença $\phi$ tal que $T$ não pode deduzir $\phi$ nem a sua negação, logo as teorias $T+\phi$ e $T+\neg \phi$ são consistentes; neste caso, dizemos que $\phi$ é independente de $T$.

Com freqüência, estaremos nos referindo às classes $\mathbb{V}$ e $\mathbb{L}$ em teoria dos conjuntos, pelo que achamos preciso lembrar fatos importantes dessas duas classes.

Definição 1.1.11. Por recursão em $\mathbb{O N}$, definimos:

$$
\begin{aligned}
& V_{0}=\varnothing \\
& V_{\alpha+1}=\wp\left(V_{\alpha}\right) \\
& V_{\alpha}=\bigcup_{\beta<\alpha} V_{\beta} \quad \text { se } \alpha \text { é um ordinal limite }
\end{aligned}
$$

Chamamos Hierarquia cumulativa de von Neumann à classe $\mathbb{V}=\bigcup_{\alpha \in \mathbb{O N}} V_{\alpha}$.

Da definição, um conjunto $A$ está em $\mathbb{V}$ se e somente se todos os seus elementos estão em $\mathbb{V}$; em particular, cada $V_{\alpha}$ é um conjunto transitivo. É claro também que para cada $\alpha \in \mathbb{O N}$, $\alpha \in V_{\alpha+1}$.

De acordo com o axioma de Regularidade, o universo da teoria dos conjuntos é $\mathbb{V}$. Além disso, nessa classe vale a axiomática ZFC; em outras palavras, $\mathbb{V}$ é um modelo de ZFC. 
Definição 1.1.12. Dizemos que um conjunto $X$ é definível num modelo $(M, \in)$ se existem uma fórmula $\phi$ na linguagem $\{\in\}$ e $a_{1}, \ldots, a_{n} \in M$ tais que $X=\left\{x \in M:(M, \in) \vDash \phi\left(x ; a_{1}, \ldots, a_{n}\right)\right\}$. Escrevemos $\operatorname{def}(M)=\{X \subseteq M: X$ é definível em $(M, \epsilon)\}$.

Por recursão em $\mathbb{O N}$ define-se

$$
\begin{aligned}
& L_{0}=\varnothing \\
& L_{\alpha+1}=\operatorname{def}\left(L_{\alpha}\right) \\
& L_{\alpha}=\bigcup_{\beta<\alpha} L_{\beta} \quad \text { se } \alpha \text { é um ordinal limite }
\end{aligned}
$$

A classe $\mathbb{L}=\bigcup_{\alpha \in \mathbb{N}} L_{\alpha}$ denomina-se hierarquia dos conjuntos construtíveis. Note que cada $L_{\alpha}$ é um conjunto transitivo e, também, $\mathbb{L}$ constitui uma classe transitiva. Em 1938, K. Gödel mostrou que $\mathbb{L}$ é um modelo (denominado tecnicamente interno) da teoria dos conjuntos, isto é, mostrou que as interpretações da axiomática ZFC em $\mathbb{L}$ são demonstráveis, inclusive, na axiomática Zermelo-Fraenkel (ZF). Adicionalmente, a interpretação de $\mathrm{CH}$ em $\mathbb{L}$ é demonstrável em ZF. Existe uma outra condição satisfeita por $\mathbb{L}$ que faz dele um modelo particularmente importante:

Fato 1.1.13. $\mathbb{L}$ é o menor modelo transitivo de ZFC que contém $\mathbb{O N}$.

\section{Forcing}

Desde os resultados de Gödel até 1963, o trabalho em teoria dos conjuntos esteve num impasse, se bem que apareceram resultados interessantes a respeito de cardinais inacessíveis e do universo construtível $\mathbb{L}$. Uma mostra desse impasse é a descoberta por Sheperdson, no início da década de cinqüenta, de que o método dos modelos internos nunca poderia providenciar uma demonstração da independência de $\mathrm{CH}$ em relação a ZF (ou ZFC).

Em 1963, um novo método que proporciona provas de consistência foi inventado por Paul Cohen. Ao contrário do método dos modelos internos que restringe o universo, o novo método do forcing "expande" o universo. Para contornar a eventual dificuldade de expandir o universo de todos os conjuntos, o método de forcing produz expansões de modelos de conjuntos finitos de axiomas de ZFC (ou ZF), pois de acordo com os teoremas de incompletude de Gödel, ZFC não pode mostrar a existência de modelos de todos os axiomas dessa teoria -a menos que fosse inconsistente-. Assim, para obter resultados de independência, basta trabalhar com subconjuntos finitos arbitrários da axiomática. Formalmente, temos o seguinte resultado. 
Teorema 1.1.14 (Princípio de forcing). Seja $\varphi$ uma fórmula. Se $\phi_{1}, \ldots, \phi_{n}$ são fórmulas de $\mathrm{ZFC}+\varphi$, existem fórmulas $\psi_{1}, \ldots, \psi_{m}$ de ZFC tais que para cada modelo transitivo enumerável $M$ satisfazendo $M \vDash\left\{\psi_{1}, \ldots, \psi_{m}\right\}$, existe um modelo transitivo enumerável $N$ tal que $N \vDash$ $\left\{\phi_{1}, \ldots, \phi_{n}\right\}$ e $M \subseteq N$. Daí, se ZFC é consistente, então a teoria $\mathrm{ZFC}+\varphi$ também é consistente.

O forcing permite construir a partir de um modelo transitivo $M$ de ZFC um modelo $N$ tal que $M \subsetneq N$ e $O \mathbb{N}^{M}=\mathbb{O N}^{N}$; desta maneira, se $\lambda=\mathbb{O N}^{M}$ temos que $L_{\lambda} \subset M \subsetneq N$ de onde $N$ é um modelo onde $\mathbb{V} \neq \mathbb{L}$. Mais ainda, todos os conjuntos que acrescentamos a $M$ para obter $N$ não são construtíveis em $N$. A grande potência desta teoria reside no fato de que as propriedades da extensão $N$ estão completamente determinadas por $M$, em outras palavras, um objeto em $M$ estará em condições de determinar o que acontece em $N$ embora não "veja" boa parte dos conjuntos de $N$.

\section{A relação de forcing}

Seja $M$ um modelo transitivo e consideremos um conjunto parcialmente ordenado (na realidade, uma pré-ordem $(\mathbb{P}, \leq)$ em $M$. Ao longo desta seção, assumiremos adicionalmente que $\mathbb{P}$ possui um maior elemento denotado por $1_{\mathbb{P}}$. No contexto do forcing, geralmente, os elementos de uma ordem parcial são chamados de condições. Se $p \leq q$ dizemos que $p$ estende $q$.

Definição 1.1.15. Dizemos que $p, q \in \mathbb{P}$ são incomparáveis se não vale $p \leq q$ nem $q \leq p$ e que são incompatíveis $(p \perp q)$ se não existe $r \in \mathbb{P}$ tal que $r \leq p$ e $r \leq q$. Uma anticadeia $A \subseteq \mathbb{P}$ é um subconjunto de elementos de $\mathbb{P}$ que são dois a dois incompatíveis.

Definição 1.1.16. Um subconjunto $D \subseteq \mathbb{P}$ é denso em $\mathbb{P}$ se e somente se para cada $p \in \mathbb{P}$ existe $q \in D$ tal que $q \leq p$, isto é, se para cada elemento da ordem existe um elemento em $D$ que forneça mais informação. De maneira similar, $D$ diz-se denso abaixo de $p \in \mathbb{P}$ se $D$ é denso na ordem parcial $\{q \in \mathbb{P}: q \leq p\}$.

Definição 1.1.17. $\mathbb{G} \subseteq \mathbb{P}$ é dito um filtro em $\mathbb{P}$ se e somente se:

1. Para todo $p \in \mathbb{G}$, se $p \leq q$ então $q \in \mathbb{G}$;

2. para todos $p$ e $q$ em $\mathbb{G}$, existe $r \in \mathbb{G}$ tal que $r \leq p$ e $r \leq q$.

Se $\mathcal{D}$ é uma família de subconjuntos densos de $\mathbb{P}$ e $\mathbb{G} \subseteq \mathbb{P}$ é um filtro tal que $D \cap \mathbb{G} \neq \varnothing$ para cada $D \in \mathcal{D}$, $\mathbb{G}$ é chamado filtro $\mathcal{D}$-genérico. Um filtro que intersecta todos os subconjuntos 
densos da ordem $\mathbb{P}$ é chamado de $\mathbb{P}$-genérico. Se a ordem parcial $\mathbb{P}$ pertence a um modelo transitivo $M$, dizemos que $\mathbb{G} \subseteq \mathbb{P}$ é $\mathbb{P}$-genérico sobre $M$ se $\mathbb{G}$ é um filtro sobre $\mathbb{P}$ e $\mathbb{G}$ intersecta todos os subconjuntos densos em $\mathbb{P}$ que são membros de $M$.

Dada uma ordem parcial $\mathbb{P}$ e uma coleção enumerável $\mathcal{D}=\left\{D_{n}\right\}_{n \in \omega}$ de subconjuntos densos em $\mathbb{P}$, sempre existe um filtro $\mathcal{D}$-genérico. Desta simples observação, obtém-se o seguinte resultado:

Proposição 1.1.18. Se $\mathbb{M}$ é um modelo transitivo enumerável, $\mathbb{P} \in \mathbb{M}$ é uma ordem parcial e $p \in \mathbb{P}$, então existe um filtro $\mathbb{G}, \mathbb{P}$-genérico sobre $\mathbb{M}$ tal que $p \in \mathbb{G}$.

Em geral, um filtro genérico sobre $M$ não está no modelo; no entanto, se $\mathbb{G}$ é $\mathbb{P}$-genérico sobre um modelo $M$, existe uma extensão minimal transitiva de $M$ que contém $\mathbb{G}$, que denotaremos por $M[\mathbb{G}]$. Nesse contexto, $M$ denomina-se o modelo base, entanto $M[\mathbb{G}]$ é chamada de extensão genérica. Uma caraterística interessante dessa extensão é a possibilidade de fazer uma descrição "interna" isto é, feita dentro do modelo $M$, dos elementos de $M[\mathbb{G}]$. Para isto, é introduzido um conjunto de nomes em $M$ que proporcionam informação sobre a maneira em que os objetos em $M[\mathbb{G}]$ foram construídos a partir de $\mathbb{P}$. Note-se que a seguinte definição é independente do modelo $M$ :

Definição 1.1.19. Seja $\mathbb{P}$ uma ordem parcial. Um conjunto $\tau$ é um $\mathbb{P}$-nome se e somente se $\tau$ é uma relação e para todo par $\langle\sigma, p\rangle \in \tau$, $\sigma$ é um $\mathbb{P}$-nome. Denotamos o conjunto de todos os $\mathbb{P}$-nomes por $\mathbb{V}^{\mathbb{P}}$.

Se $M$ é um modelo contendo $\mathbb{P}$ denotamos por $M^{\mathbb{P}}=M \cap \mathbb{V}^{\mathbb{P}}$ o conjunto de todos os $\mathbb{P}$-nomes em $M$. Se $\mathbb{G}$ é um $\mathbb{P}$-genérico sobre $M$, a interpretação (ou valor) de um $\mathbb{P}$-nome $\tau \in M^{\mathbb{P}}$, denotada como $\tau_{\mathbb{G}}$, define-se recursivamente:

$$
\tau_{\mathbb{G}}=\left\{\sigma_{G}:(\exists p \in \mathbb{G})\langle\sigma, p\rangle \in \tau\right\}
$$

Seja $M[\mathbb{G}]=\left\{\tau_{\mathbb{G}}: \tau \in M^{\mathbb{P}}\right\}$. Como $\dot{\mathbb{G}}=\{\langle p, p\rangle: p \in \mathbb{G}\}$ é um $\mathbb{P}$-nome para $\mathbb{G}$, temos $\mathbb{G} \in M[\mathbb{G}]$.

Definição 1.1.20. Sejam $M$ um modelo transitivo enumerável, $\mathbb{P}$ uma ordem parcial em $M$ e $p \in \mathbb{P}$. Se $\varphi\left(x_{1}, \ldots, x_{n}\right)$ é uma fórmula e $\tau_{1}, \ldots, \tau_{n} \in M^{\mathbb{P}}$, dizemos que $p$ força $\varphi\left(\tau_{1}, \ldots, \tau_{n}\right)$, em símbolos $p \Vdash \varphi\left(\tau_{1}, \ldots, \tau_{n}\right)$ se e somente se para todo filtro $\mathbb{P}$-genérico $\mathbb{G}$ sobre $M$ tal que $p \in \mathbb{G}$ vale:

$$
M[\mathbb{G}] \models \varphi\left(\left(\tau_{1}\right)_{\mathbb{G}}, \ldots,\left(\tau_{n}\right)_{\mathbb{G}}\right)
$$

Ao fazer menção do modelo, da extensão e do filtro genérico, salienta-se que a relação de forcing está definida fora do próprio modelo $M$. No entanto, a definição mais formal -e 
naturalmente mais forte- é feita internamente por meio da relação $\mathbb{}^{*}$ (vide [47]). A definição interna nos permite ver que para uma fórmula $\varphi$ e quaisquer $\mathbb{P}$-nomes em $M^{\mathbb{P}}$, o conjunto $\left\{p \in \mathbb{P}: p \Vdash \varphi\left(\tau_{1}, \ldots, \tau_{n}\right)\right\}$ está no modelo $M$, e assim pode ser decidido em $M$ se $p \Vdash \varphi$ ou não.

Lema 1.1.21 (Lema da verdade para extensões genéricas). Sejam $M$ um modelo enumerável transitivo e $\mathbb{P}$ uma ordem parcial em $M$. Se $\mathbb{G}$ é $\mathbb{P}$-genérico sobre $M, \varphi\left(x_{1}, \ldots, x_{n}\right)$ é uma fórmula $e \tau_{1}, \ldots, \tau_{n} \in M^{\mathbb{P}}$, então

$$
\left(M[G] \vDash \varphi\left(\left(\tau_{1}\right)_{\mathbb{G}}, \ldots,\left(\tau_{n}\right)_{\mathbb{G}}\right)\right) \leftrightarrow(\exists p \in \mathbb{G})\left(p \Vdash \varphi\left(\tau_{1}, \ldots, \tau_{n}\right)\right)
$$

Demonstração. Vide [47], VII. § 3.5.

Foi apontado que os ordinais em $M$ e na extensão genérica $M[\mathbb{G}]$ são os mesmos; no entanto, os cardinais em $M$ e $M[\mathbb{G}]$ não necessariamente coincidem pois a noção de cardinalidade não é absoluta.

Definição 1.1.22. Seja $M$ um modelo transitivo enumerável e seja $\mathbb{P}$ uma ordem parcial em $M$. Dizemos que $\mathbb{P}$ preserva cardinais se e somente se para qualquer $\mathbb{P}$-genérico $\mathbb{G}$ sobre $M$, temos que

$$
\forall \alpha \in \mathbb{O N}^{M}\left((\alpha \text { é cardinal })^{M} \leftrightarrow(\alpha \text { é cardinal })^{M[\Im]}\right)
$$

Dizemos também, que $\mathbb{P}$ preserva cofinalidades se e somente se para cada ordinal limite $\gamma \in \mathbb{O N}^{M}$, temos $\operatorname{cf}(\gamma)^{M}=\operatorname{cf}(\gamma)^{M[\mathbb{G}]}$.

As noções de preservar cardinais e cofinalidades abaixo e acima de um cardinal $\alpha \in M$ ou um ordinal $\gamma \in \mathbb{O N}^{M}$, são definidas de maneira análoga.

Fato 1.1.23. Sejam $M$ um modelo transitivo enumerável, $\mathbb{P} \in M$ uma ordem parcial. $\mathbb{P}$ preserva cardinais se e somente se preserva cofinalidades.

Demonstração. Vide [47].

Definição 1.1.24. Uma ordem parcial $\mathbb{P}$ satisfaz a condição de cadeia enumerável (c.c.c.) se toda anticadeia de elementos de $\mathbb{P}$ é enumerável. Em geral, se $\kappa$ é um cardinal, $\mathbb{P}$ é dita $\kappa$-c.c. se e somente se toda anticadeia em $\mathbb{P}$ tem cardinalidade menor do que $\kappa$ (daí que c.c.c. é $\boldsymbol{\aleph}_{1}$-c.c.).

O seguinte resultado, mostra a relação da propriedade anterior com a preservação de cardinais em extensões genéricas. 
Fato 1.1.25. Sejam $M$ um modelo transitivo enumerável, $\mathbb{P} \in M$ uma ordem parcial e $\kappa$ um cardinal de $M$. Se $(\mathbb{P} \text { é } \kappa \text {-c.c. })^{M}$, então $\mathbb{P}$ preserva cofinalidades acima de $\kappa$; se adicionalmente $(\kappa \text { é regular })^{M}$, então $\mathbb{P}$ preserva cardinais acima de $\kappa$. Em particular, se $(\mathbb{P} \text { é c.c.c. })^{M}$ então $\mathbb{P}$ preserva cofinalidades (e assim cardinalidades).

Definição 1.1.26. Se $\lambda$ é um cardinal, uma ordem parcial $\mathbb{P}$ é dita $\lambda$-fechada se para cada $\gamma<\lambda$, e cada seqüência decrescente $\left\{p_{\xi}: \xi<\gamma\right\} \subseteq \mathbb{P}$ existe $p \in \mathbb{P}$ tal que $p \leq p_{\xi}$ para cada $\xi<\gamma$.

Podemos agora mencionar outro fato no que se refere a preservação de cardinais.

Fato 1.1.27. Sejam $M$ um modelo transitivo enumerável, $\mathbb{P} \in M$ uma ordem parcial e $\kappa$ um cardinal em $M$. Se ( $\mathbb{P}$ é $\kappa-f e c h a d a)^{M}$, então $\mathbb{P}$ preserva cofinalidades (e assim cardinais) abaixo de $\kappa$.

A continuação, apresentam-se algumas noções de forcing cujos elementos genéricos produzem um número real.

Exemplo 1.1.28 (Forcing de Cohen). Sejam $I$ um conjunto infinito e $J$ um conjunto. Consideremos o conjunto $\mathrm{Fn}(I, J)$ com a ordem $\leq$ onde $s \leq t$ se e somente se $s \supseteq t$. A ordem parcial $\mathbb{C}_{I}=(\operatorname{Fn}(I, 2), \preceq)$ denomina-se forcing de Cohen e constitui uma ordem c.c.c. Uma extensão genérica obtida por meio da ordem $\mathbb{C}_{I}$ acrescenta uma nova função $c_{I} \in 2^{I}$ dada por $c_{I}=\bigcup\left\{s \in C_{I}: s \in \mathbb{G}\right\}$, onde $\mathbb{G}$ é um filtro genérico sobre um modelo base $M$. É bem conhecido que $\mathbb{G}$ pode ser reconstruído usando $c_{I}$, isto é, $M\left[c_{I}\right]=M[\mathbb{G}]$. O elemento $c_{I}$ chama-se de função de Cohen e, quando $I=\omega, c=c_{\omega}$ denomina-se real de Cohen. Esta noção de forcing é particularmente importante pois pode-se mostrar que qualquer noção de forcing enumerável é equivalente a $\mathbb{C}_{\omega}$.

Exemplo 1.1.29 (Forcing de Sacks). Consideremos o conjunto parcialmente ordenado $\left(2^{<\omega}, \subseteq\right)$. Um subconjunto $T \subseteq 2^{<\omega}$ é dito uma árvore se para cada $t \in T$, o segmento inicial $\{s \in T$ : $s \subseteq t$ \} é bem ordenado pela relação $\subseteq$. Uma árvore não vazia $T \subseteq 2^{<\omega}$ é dita perfeita se para cada $t \in T$ existe uma extensão $s \supseteq t$ tal que $s^{\frown} 0 \in T$ e $s^{\frown} 1 \in T$. O forcing de Sacks $\mathbb{S}$ consiste do conjunto de todas as árvores perfeitas em $2^{<\omega}$ ordenadas por inclusão, isto é, $S \leq T$ se e somente se $S \subseteq T$. Uma extensão genérica obtida mediante essa ordem, acrescenta um novo real $s$, onde $\{s\}=\bigcap\{[T]: T \in \mathbb{G}\},[T]=\left\{s \in 2^{<\omega}: s \uparrow_{n} \in T\right.$ para todo $\left.n \in \omega\right\}$ e $\mathbb{G}$ é um filtro $\mathbb{S}$-genérico sobre um modelo $M$. Nesta situação, $s$ é chamado de real de Sacks. Como antes, também vale que $M[s]=M[\mathbb{G}]$. 
Pode-se mostrar que cada árvore perfeita cinde numa coleção $\left\{T_{\alpha}: \alpha<\mathfrak{c}\right\}$ de $\mathfrak{c}$ árvores perfeitas, e para quaisquer $T_{\alpha}$ e $T_{\beta}$ distintas, $T_{\alpha} \cap T_{\beta}$ é um conjunto finito. Em particular, se $\alpha \neq \beta$ então $\left[T_{\alpha}\right] \cap\left[T_{\beta}\right]=\varnothing$ e $T_{\alpha} \perp T_{\beta}$, ou seja, $\mathbb{S}$ não é c-c.c; no entanto, como $|\mathbb{S}|=\mathfrak{c}$, obtemos que $\mathbb{S}$ é $\mathfrak{c}^{+}$-c.c. Assim, assumindo $\mathrm{CH}, \mathbb{S}$ é $\boldsymbol{\aleph}_{2}$-c.c. e preserva cardinais acima de $\boldsymbol{\aleph}_{2}$. Torna-se, então, uma questão importante a preservação de $\aleph_{1}$ em $\mathbb{S}$. Esta é obtida pelo denominado argumento de fusão, técnica bastante comum para noções de forcing cujas condições são árvores. Os detalhes a respeito, podem-se achar em [44] 115 .

Para mostrar a consistência de $\mathrm{ZFC}+\neg \mathrm{CH}$, é preciso acrescentar a um modelo transitivo enumerável $M$ de ZFC uma grande quantidade de reais; uma maneira efetiva de resolver esse problema é usando produtos de ordens.

Definição 1.1.30. Se $\left(\mathbb{P}, \leq_{\mathbb{P}}\right)$ e $\left(\mathbb{Q}, \leq_{\mathbb{Q}}\right)$ são ordens parciais, define-se sobre o produto cartesiano $\mathbb{P} \times \mathbb{Q}$ a ordem $\leq$, onde $\left(p^{\prime}, q^{\prime}\right) \leq(p, q)$ se e somente se $p^{\prime} \leq_{\mathbb{P}} p$ e $q^{\prime} \leq_{\mathbb{P}} q$.

Lema 1.1.31 (Lema do Produto). Se $\left(\mathbb{P}, \leq_{\mathbb{P}}\right) e\left(\mathbb{Q}, \leq_{Q}\right)$ são ordens parciais num modelo transitivo enumerável $M$, então $\mathbb{G} \subseteq \mathbb{P} \times \mathbb{Q}$ é genérico sobre $M$ se e somente se $G=G_{1} \times G_{2}$ onde $\mathbb{G}_{1} \subseteq \mathbb{P}$ é $\mathbb{P}$-genérico sobre $M e \mathbb{G}_{2} \subseteq \mathbb{Q}$ é $\mathbb{Q}$-genérico sobre $M\left[\mathbb{G}_{1}\right]$. Nesse caso, $M[\mathbb{G}]=M\left[\mathbb{G}_{1}\right]\left[\mathbb{G}_{2}\right]=$ $M\left[\mathbb{G}_{2}\right]\left[\mathbb{G}_{1}\right]$.

Exemplo 1.1.32 (O forcing de Easton). Uma função de Easton é uma função $E$ tal que dom $E$ é um conjunto de cardinais regulares satisfazendo

i. para cada $\kappa \in \operatorname{dom} E, E(\kappa)$ é um cardinal e $\operatorname{cf}(E(\kappa))>\kappa$;

ii. para cada $\kappa, \mu \in \operatorname{dom} E$, se $\kappa<\mu$ então $E(\kappa) \leq E(\mu)$.

De acordo com observações precedentes, note-se que uma função de Easton é simplesmente uma função que comparte as propriedades da função do contínuo sobre os cardinais regulares. Se $E$ é uma função de Easton, o produto de Easton associado a $E$ define-se como o conjunto

$$
\mathbb{P}(E)=\left\{p \in \prod_{\kappa \in \operatorname{dom} E} \operatorname{Fn}(E(\kappa), 2):|p|<\kappa\right\}
$$

ordenado pela relação $p \leq q \leftrightarrow \forall \kappa \in \operatorname{dom} E(q(\kappa) \subseteq p(\kappa))$.

Numa extensão genérica obtida por meio de um produto de Easton, a função do contínuo coincide com a correspondente função de Easton no domínio dela; formalmente, temos o seguinte resultado cuja prova encontra-se em [47] VIII, §4: 
Fato 1.1.33. Sejam M um modelo transitivo enumerável satisfazendo $\mathrm{GCH}, E$ é uma função de Easton em $M$ e $\mathbb{P}=\mathbb{P}(E)$. Então $\mathbb{P}$ preserva cofinalidades (e assim cardinais) e, se $\mathbb{G}$ é $\mathbb{P}$-genérico sobre $M$, então $(\forall \kappa \in \operatorname{dom} E)\left(2^{\kappa}=E(\kappa)\right)^{M[G]}$. Mais geralmente, se $\theta$ é um cardinal infinito em $M[\mathbb{G}], \operatorname{sejam} E^{\prime}(\theta)=\max \left\{\theta^{+}, \sup \{E(\kappa): \kappa \in \operatorname{dom} E, \kappa \leq \theta\}\right\}$ e $E^{*}(\theta)=E^{\prime}(\theta) \operatorname{se} \operatorname{cf}\left(E^{\prime}(\theta)\right)>\theta$ ou $E^{*}(\theta)=E^{\prime}(\theta)^{+}$em caso contrário. Então, $2^{\theta}=E^{*}(\theta)$.

Assim, é consistente com ZFC qualquer determinação da função do contínuo sobre os cardinais regulares sempre que satisfizer a monotonia e a desigualdade de Zermelo-König.

Para os nossos fins, convém precisar alguns fatos sobre forcing iterado.

Definição 1.1.34. Dadas $\mathbb{P}$ uma noção de forcing, e $\mathbb{Q}$ um $\mathbb{P}$-nome para uma noção de forcing, dizemos que

$$
\mathbb{P} * \dot{\mathbb{Q}}=\left\{(p, \dot{q}): p \in \mathbb{P} \text { e } \Vdash_{\mathbb{P}} \dot{q} \in \dot{Q}\right\}
$$

é a iteração de forcing de dois passos de $\mathbb{P}$ e $\mathbb{Q}$ onde $(p, \dot{q}) \leq\left(p^{\prime}, \dot{q}^{\prime}\right)$ se e somente se $p \leq_{\mathbb{P}} p^{\prime}$ e $p^{\prime} \mathbb{1}_{\mathbb{P}} \dot{q} \leq_{Q} \dot{q}^{\prime}$.

É bem conhecido que forçar com $\mathbb{P} * \mathbb{Q}$ produz o mesmo resultado do que forçar primeiro com $\mathbb{P}$ sobre um modelo $M$ e depois forçar com $\mathbb{Q}$ na extensão $M[\mathbb{G}]$.

Definição 1.1.35. Seja $\delta \in \mathbb{O N}$. Uma iteração de comprimento $\delta$ de forcing com limite $\mathbb{P}_{\delta}$, é uma sequência $\left\{\left(\mathbb{P}_{\alpha}, \dot{\mathbb{Q}}_{\alpha}\right): \alpha<\delta\right\}$ onde para cada $\alpha \leq \delta, \mathbb{P}_{\alpha}$ é uma noção de forcing que consiste de funções com domínio $\alpha$ satisfazendo

\section{i. $\mathbb{P}_{0}=\{\varnothing\}$}

ii. $\dot{\mathbb{Q}}_{\alpha}$ é um $\mathbb{P}_{\alpha}$-nome para uma ordem parcial, $\mathbb{P}_{\alpha+1} \cong \mathbb{P}_{\alpha} * \dot{\mathbb{Q}}_{\alpha}$ e para cada $p, q \in \mathbb{P}_{\alpha+1}, q \leq_{\alpha+1} p$ se $q \uparrow_{\alpha} \leq_{\alpha} p \uparrow_{\alpha}$ e $q \uparrow_{\alpha} \Vdash_{\alpha} q(\alpha) \leq_{\dot{Q}_{\alpha}} p(\alpha)$,

iii. se $\beta \leq \delta$ é um ordinal limite, seja $\mathbb{P}_{\beta}=\left\{p: \exists \alpha<\beta\left(\operatorname{supp}(p) \subseteq \alpha\right.\right.$ e $\left.\left.p \uparrow_{\alpha} \in \mathbb{P}_{\alpha}\right)\right\}$ ou $\mathbb{P}_{\beta}=\left\{p: \forall \alpha<\beta p \uparrow_{\alpha} \in \mathbb{P}_{\alpha}\right\} ;$ se $p, q \in \mathbb{P}_{\beta}, q \leq_{\beta} p$ se para cada $\alpha<\beta, q \uparrow_{\alpha} \leq_{\alpha} p \uparrow_{\alpha}$.

onde $\operatorname{supp}(p)=\left\{\alpha: p(\alpha) \neq 1_{\dot{Q}_{\alpha}}\right\}$ denota o suporte de $p$.

Uma iteração de forcing $\mathbb{P}_{\delta}$ denomina-se de suporte finito (enumerável) se para todo $p \in \mathbb{P}_{\delta}$, $|\operatorname{supp}(p)|<\omega(|\operatorname{supp}(p)| \leq \omega)$.

Exemplo 1.1.36 (Forcing side-by-side de Sacks). Seja $\mathbb{S}$ o forcing de Sacks definido em 1.1.29. Pode-se mostrar que um produto (ou iteração) de suporte finito da noção de forcing $\mathbb{S}$ colapsa 
cardinais. No entanto, é possível aumentar o tamanho do contínuo acrescentando um número arbitrário $\kappa$ de reais de Sacks simultaneamente mediante a noção de forcing $\mathbb{S}_{\omega}^{\kappa}$, cujas condições são os elementos com suporte enumerável infinito do conjunto $\mathbb{S}^{\kappa}$ considerando a ordem $\leq$ onde $f \leq g$ se e somente se $f(\xi) \leq g(\xi)$ para cada $\xi<\kappa$. A noção de forcing $\mathbb{S}_{\omega}^{\kappa}$ é conhecida como forcing side-by-side de Sacks. Note-se que os reais de Sacks acrescentados correspondem com as coordenadas de uma função obtida por meio de um filtro genérico. De maneira similar à noção de forcing $\mathbb{S}, \mathbb{S}_{\omega}^{\kappa}$ preserva $\boldsymbol{\aleph}_{1}$ e, assumindo $\mathrm{CH}$, preserva todos os cardinais. Se adicionalmente, no modelo base $M$ tem-se $\kappa^{\omega}=\kappa$, então $(\mathfrak{c}=\kappa)^{M[\mathbb{G}]}$ onde $\mathbb{G}$ é $\mathbb{S}_{\omega}^{\kappa}$-genérico para $M$.

Exemplo 1.1.37 (Forcing iterado de Sacks). Em contraste com o anterior exemplo, Baumgartner e Laver [7] optaram por aumentar o tamanho do contínuo acrescentando reais de Sacks iterativamente, onde cada real é um elemento genérico sobre o modelo obtido após ter acrescentando outros reais. O trabalho desses autores mostra que uma iteração de comprimento $\alpha$ de suporte enumerável infinito da ordem $\mathbb{S}, \mathbb{S}_{\alpha}$, não colapsa $\omega_{1}$ (pois resulta ser $\omega_{1}$-fechada) e partindo de um modelo de $\mathrm{CH}$, para cada $\alpha \leq \omega_{2}$ a ordem $\mathbb{S}_{\alpha}$ satisfaz a $\boldsymbol{\aleph}_{2}$-c.c. e assim, preserva cardinais. Nesse caso, no modelo extensão temos $\mathfrak{c}=\boldsymbol{\aleph}_{2}$.

\section{O Axioma de Martin}

Definição 1.1.38. Para cada cardinal $\kappa$, o Axioma de Martin para $\kappa$ é a afirmação

MA(א): Se $\mathbb{P}$ é uma ordem parcial c.c.c. e $\mathcal{D}$ é uma família de subconjuntos densos em $\mathbb{P}$ tal que $|\mathcal{D}| \leq \kappa$ então existe um filtro $\mathcal{D}$-genérico $\mathbb{G} \subseteq \mathbb{P}$.

O Axioma de Martin é a afirmação

$$
\text { MA: } \forall\left(\kappa<2^{\kappa_{0}}\right)(M A(\kappa))
$$

É bem conhecido que $\mathrm{MA}(\omega)$ é verdadeiro e que $\mathrm{MA}\left(2^{\mathrm{N}_{0}}\right)$ é falso. Naturalmente, $\mathrm{CH}$ implica MA e ainda, MA é relativamente consistente com $\mathrm{ZFC}+\neg \mathrm{CH}$.

Existem maneira alternativas de enunciar o Axioma de Martin, entre as quais, destaca uma versão topológica que será usada em algumas partes do texto. Antes de enuncia-la, convém precisar alguns termos.

Definição 1.1.39. Seja $(X, \tau)$ um espaço topológico e seja $\mathbb{P}_{\tau}=\{p \in \tau: p \neq \varnothing\}$. Em $\mathbb{P}_{\tau}$, considere-se a relação $p \leq q \leftrightarrow p \subseteq q$. Dizemos que $(X, \tau)$ satisfaz a condição de cadeia enumerável se a ordem associada a $\mathbb{P}_{\tau}$ é c.c.c. 
Note-se que em $\mathbb{P}_{\tau}, p \perp q$ se e somente se $p \cap q=\varnothing$; assim, $\mathbb{P}$ e portanto $(X, \tau)$ satisfaz c.c.c. se e somente se qualquer coleção de abertos não vazios em $X$ dois a dois disjuntos é enumerável.

Fato 1.1.40. Para cada $\kappa \geq \omega$, as seguintes afirmações são equivalentes:

a. $\operatorname{MA}(\kappa)$

b. $\mathrm{MA}(\kappa)$ restrito às ordens parciais $\mathbb{P}$ satisfazendo $|\mathbb{P}| \leq \kappa$.

c. Se X é um espaço topológico Hausdorff compacto c.c.c. $e\left\{U_{\alpha}: \alpha<\kappa\right\}$ é uma coleção de abertos densos em $X$, então $\bigcap_{\alpha<\kappa} U_{\alpha} \neq \varnothing$.

Do anterior, segue que MA é equivalente à asserção: "Em cada espaço Hausdorff compacto satisfazendo c.c.c. a interseção de uma coleção de uma quantidade menor do que $2^{\aleph_{0}}$ conjuntos densos é denso".

Fato 1.1.41. Para cada $\omega<\kappa<\mathfrak{c}$, a afirmação MA(א) implica $2^{\kappa}=2^{\omega}=\mathfrak{c}$. Assim, sob MA, $\mathfrak{c}$ é um cardinal regular.

\section{MA restrito a alguns tipos de ordens parciais}

Duas versões mais fracas de MA serão usadas no texto: o Axioma de Martin restrito às ordens $\sigma$-centradas e o Axioma de Martin restrito às ordens parciais enumeráveis.

Definição 1.1.42. Um subconjunto $Q$ de uma ordem parcial $\mathbb{P}$ diz-se centrado se e somente se cada subconjunto finito $\left\{p_{1}, \ldots, p_{n}\right\} \subseteq Q$ possui um minorante. Note que o elemento minorante não necessariamente pertence a $Q$.

Definição 1.1.43. Uma ordem parcial $\mathbb{P}$ diz-se $\lambda$-centrada se e somente se é reunião de $\lambda$ subconjuntos centrados. Uma ordem parcial $\aleph_{1}$-centrada denomina-se $\sigma$-centrada.

Representamos por $\mathrm{MA}_{\sigma \text {-centrada }}$ a asserção MA restrita a ordens $\sigma$-centradas. Salienta-se que $\mathrm{MA}_{\sigma \text {-centrada }}$ é uma afirmação estritamente mais fraca do que MA [48] e é equivalente ao princípio combinatório $P(\mathfrak{c})$ [9].

Por outro lado, $\mathrm{MA}_{\text {enumerável }}$ vai representar a asserção MA restrita a ordens parciais enumeráveis. $\mathrm{MA}_{\text {enumerável }}$ é uma afirmação estritamente mais fraca do que $\mathrm{MA}_{\sigma \text {-centrada }}$ e é equivalente à afirmação

$\mathrm{MA}_{\text {enumerável }}$ : "A interseção de menos do que c abertos densos na reta real $\mathbb{R}$ é um conjunto denso" 
Nota 1.1.44. Muitas vezes, resulta conveniente usar $M A_{\text {enumerável }}$ do que outras versões mais fortes de MA. A razão consiste em que esta asserção não impõe restrições sobre a cardinalidade do continuo $\mathfrak{c}$; de fato, $\mathrm{MA}_{\sigma \text {-centrada }}$ implica que para cada $\omega \leq \kappa<\mathfrak{c}, 2^{\kappa}=\mathfrak{c}$ de onde $\mathfrak{c}$ é regular, enquanto $\mathrm{MA}_{\text {enumerável }}$ é consistente com qualquer conduta cardinal permissível. Por exemplo, após acrescentar $\boldsymbol{\aleph}_{\omega_{1}}$ reais de Cohen a um modelo $M$ de $\mathrm{ZFC}+\mathrm{CH}$, na extensão genérica temos que $\mathfrak{c}=\boldsymbol{\aleph}_{\omega_{1}}$ e $M A_{\text {enumerável }}$ é satisfeito. Ainda mais, $M_{\text {enumerável vale em }}$ qualquer modelo de ZFC obtido acrescentando reais de Cohen a um modelo de ZFC+GCH [8].

Dado que muitas construções a serem apresentadas usam forcing ou MA, com freqüência será preciso mostrar que uma ordem parcial dada é c.c.c., para o qual, usualmente tomamos um subconjunto não enumerável da ordem que iremos refinando até obter um par de elementos compatíveis. Por tal motivo, inclui-se neste ponto o lema do $\Delta$-sistema, uma ferramenta combinatória eficaz para provar tais resultados.

Definição 1.1.45. Uma família $\mathcal{F}$ de conjuntos denomina-se um $\Delta$-sistema ou uma família quase-disjunta de raiz $r$ se e somente se para dois membros distintos $x, y \in \mathcal{F}$, tem-se $x \cap y=r$.

Teorema 1.1.46 (Lema do $\Delta$-sistema). Seja $\kappa$ um cardinal infinito e seja $\theta>\kappa$ um cardinal regular satisfazendo que $\forall \alpha<\theta,\left|\alpha^{<\kappa}\right|<\theta$. Suponhamos ainda que $\mathcal{A}$ é uma família de conjuntos tal que $|\mathcal{A}| \geq \theta$ e para cada $a \in \mathcal{A},|a|<\kappa$. Então existe $\mathcal{B} \subseteq \mathcal{A}$ tal que $|\mathcal{B}|=\theta$ e $\mathcal{B}$ forma um $\Delta$-sistema.

Contudo, a versão do lema do $\Delta$-sistema que usaremos mais freqüentemente é um caso particular do teorema anterior, tomando $\kappa=\omega$ e $\theta=\omega_{1}$ : "Se $\mathcal{A}$ é uma família não enumerável de conjuntos finitos, então existe um $\Delta$-sistema $\mathcal{B} \subset \mathcal{A}$ não enumerável".

\subsection{Topologia}

Denotaremos um espaço topológico por $(X, \tau)$ onde $X$ é o conjunto suporte e $\tau$ é a topologia definida nesse conjunto. Geralmente, escreveremos simplesmente $X$ ao invés de $(X, \tau)$ se não houver dúvida a respeito da topologia considerada. Para os temas relacionados com topologia geral, seguiremos a referência [29]. Assumimos certa familiaridade do leitor com os conceitos básicos de topologia geral.

Fixemos $(X, \tau)$ um espaço topológico.

Se $Y \subseteq X$, consideraremos em $Y$ a topologia de subespaço $\tau^{Y}=\{U \cap Y: U \in \tau\}$. Dado $x \in X$, adotaremos a notação $\tau^{x}=\{U \in \tau: x \in U\}$. Se $A \subseteq X$, representamos o 
fecho e o interior de $A$ em $X$ por $\bar{A}^{X}$ e $\operatorname{int}(A)_{X}$ respetivamente. Quando é claro o espaço considerado, escreveremos $\bar{A} \mathrm{e} \operatorname{int}(A)$ simplesmente. O conjunto de pontos de acumulação de $A$ é representado pelo símbolo $A^{d}$.

Como de praxe, representamos os axiomas de separação mediante o símbolo $T_{i}$ para cada $i=0,1,2,3,3 \frac{1}{2}$, 4 . Um espaço $X$ é denominado regular se é $T_{1}$ e $T_{3}$, de Tychonoff ou completamente regular se é $T_{1}$ e $T_{3 \frac{1}{2}}$, e normal se ele é $T_{1}$ e $T_{4}$.

Se $\left\{X_{s}: s \in S\right\}$ é uma família de espaços topológicos, consideraremos no produto cartesiano $\prod_{s \in S} X_{s}$ a topologia produto de Tychonoff; para cada $s \in S$ denotaremos por $\pi_{s}: \prod_{t \in S} X_{t} \rightarrow X_{s}$ a projeção sobre o $s$-ésimo fator. Se $A \subseteq S$, denotaremos ainda por $p_{A}: \prod_{s \in S} X_{s} \rightarrow \prod_{s \in A} X_{s}$ a projeção sobre o produto parcial $\prod_{s \in A} X_{s}$.

Definição 1.2.1. Um espaço topológico $X$ denomina-se diádico se é imagem contínua do cubo de Cantor generalizado $\{0,1\}^{\kappa}$ para algum cardinal $\kappa$.

Definição 1.2.2. Seja $Y$ um espaço topológico. Se para cada $s \in S, f_{s}: Y \rightarrow X_{s}$ é uma função, o produto diagonal da família $\mathcal{F}=\left\{f_{s}: s \in S\right\}$ é definido como a função $\Delta_{s \in S} f_{s}: Y \rightarrow \prod_{s \in S} X_{s}$ tal que para cada $y \in Y, \Delta_{s \in S} f_{s}(y)=\left(f_{s}(y)\right)_{s \in S}$.

Definição 1.2.3. Sob as mesmas condições na definição anterior, se cada um dos membros da família $\mathcal{F}$ é uma função contínua, dizemos que $\mathcal{F}$ separa pontos se para cada par de pontos distintos $x, y \in Y$, existe uma função $f_{s} \in \mathcal{S}$ tal que $f_{s}(x) \neq f_{s}(y)$. Dizemos que a família $\mathcal{F}$ separa pontos e conjuntos fechados se para cada $y \in Y$ e cada fechado $F \subseteq Y$ que não contém $y$, existe $s \in S$ tal que $f_{s}(y) \notin \overline{f_{s}[F]}$.

Teorema 1.2.4. Seja $\mathcal{F}=\left\{f_{s}: s \in S\right\}$ uma família de funções contínuas, onde $f_{s}: Y \rightarrow X_{s}$ para cada $s \in S$. Então o produto diagonal $f=\Delta_{s \in S} f_{s}$ é uma função contínua e, se F separa pontos, $f$ é injetora. Além disso, se $\mathcal{F}$ separa pontos e conjuntos fechados então $f$ é uma imersão topológica. Em particular, se existe $s \in S$ tal que $f_{s}$ é uma imersão topológica, $f$ também é imersão topológica.

Definição 1.2.5. Um conjunto $A \subseteq X$ é dito denso em $X$ se $\bar{A}=X$, co-denso em $X$ se $X \backslash A$ é denso, raro em $X$ se $\bar{A}$ é co-denso, e, denso em si mesmo se $A \subseteq A^{d}$.

Definição 1.2.6. Um subespaço $Y$ de um espaço topológico $X$ é $G_{\delta}$-denso em $X$ se todo subconjunto $G_{\delta}$ de X intersecta $Y$.

Definição 1.2.7. Se $X$ não tiver subespaços próprios densos em si mesmos dizemos que $X$ é disperso; se qualquer interseção enumerável de abertos densos em $X$ é um subconjunto denso em $X, X$ é dito um espaço de Baire. 


\section{Algumas propriedades relacionadas com a compacidade}

\section{Pseudocompacidade}

Em 1948, Hewitt introduziu a classe dos espaços pseudocompactos:

Definição 1.2.8. Um espaço de Tychonoff $X$ é pseudocompacto se e somente se toda função contínua $f: X \rightarrow \mathbb{R}$ é limitada.

Proposição 1.2.9. Para um espaço de Tychonoff $X$, as seguintes afirmações são equivalentes:

1. X é pseudocompacto;

2. se $\left\{W_{n}: n \in \omega\right\}$ é uma seqüência decrescente de abertos não vazios de X, então $\bigcap_{n \in \omega} \overline{W_{n}} \neq \varnothing$;

3. se $\left\{V_{n}: n \in \omega\right\}$ é uma família de subconjuntos abertos não vazios de $X$ que possui a propriedade de interseção finita, então $\bigcap_{n \in \omega} \overline{V_{n}} \neq \varnothing$;

4. se $\left\{U_{n}: n \in \omega\right\}$ é um recobrimento aberto de $X$, existe $J \in[\omega]^{<\omega}$ tal que $X=\bigcup_{j \in J} \overline{U_{j}}$.

Demonstração. Vide [29].

\section{Compacidade Enumerável}

Definição 1.2.10. Um espaço topológico $X$ é enumeravelmente compacto se toda cobertura aberta enumerável de $X$ possui um sub-recobrimento finito.

O seguinte resultado mostra algumas caraterizações úteis dos espaços enumeravelmente compactos.

Proposição 1.2.11. Se X é um espaço topológico $T_{2}$, as seguintes afirmações são equivalentes:

1. X é enumeravelmente compacto;

2. todo subconjunto enumerável infinito em $X$ tem um ponto de acumulação;

3. toda seqüência em $X$ possui um ponto de acumulação;

4. toda família localmente finita de subconjuntos não vazios de X é finita;

5. X não possui um subespaço infinito enumerável que seja fechado e discreto;

6. se $\mathcal{F}=\left\{F_{n}: n \in \omega\right\}$ é uma família de conjuntos fechados não vazios em $X$ que satisfaz a propriedade de interseção finita, então $\bigcap_{n \in \omega} F_{n} \neq \varnothing$. 
Proposição 1.2.12. A compacidade enumerável é uma propriedade hereditária para subespaços fechados, preservada sob imagens contínuas e, além disso, é uma propriedade aditiva, isto é, preservada sob soma topológica. No entanto, não constitui uma propriedade produtiva ${ }^{1}$

É bem conhecido que todo espaço de Hausdorff localmente compacto é um espaço de Baire. A respeito da propriedade de Baire, quando consideramos espaços enumeravelmente compactos, uma propriedade análoga aparece.

Proposição 1.2.13. Um espaço de Tychonoff X enumeravelmente compacto é um espaço de Baire.

\section{Algumas funções cardinais}

A seguinte definição, compreende as funções cardinais básicas:

Definição 1.2.14. Seja $(X, \tau)$ um espaço topológico.

a. O peso de $X$ é $w(X)=\min \{|\mathcal{B}|: \mathcal{B}$ é uma base de $X\}+\omega$.

b. Se $p \in X$, o caráter do ponto $p$ em $X$, é $\chi(p, X)=\min \{|\mathcal{V}|: \mathcal{V}$ é uma base local de $p\}$.

c. O caráter de $X$ é $\chi(X)=\sup \{\chi(p, X): p \in X\}+\omega$.

d. A densidade de $X$ é $d(X)=\min \{|S|: S \subseteq X$ é denso em $X\}+\omega$.

Definição 1.2.15. Uma família $\mathcal{C} \subseteq \tau \backslash\{\varnothing\}$ é dita celular se os elementos dela são dois a dois disjuntos. O cardinal $c(X)=\sup \{|\mathfrak{C}|: \mathcal{C}$ é celular em $X\}+\omega$ chama-se de celularidade de $X$.

A seguinte proposição, reúne certas desigualdades envolvendo os invariantes cardinais acima mencionados.

Proposição 1.2.16. Seja $(X, \tau)$ um espaço topológico $T_{2}$. Então,
a. $w(X) \leq 2^{|X|}$;
b. $|X| \leq 2^{w(X)}$;
c. $c(X) \leq d(X)$;
d. $|\tau| \leq 2^{w(X)}$
e. $w(X) \leq 2^{d(X)}$;

${ }^{1}$ Vide [29], p. 205-206. 
f. $|X| \leq d(X)^{\chi(X)}$

g. $|X| \leq 2^{c(X) \chi(X)}$.

Teorema 1.2.17 (Teorema de Hewitt-Marczewski-Pondiczery). Seja $\left\{X_{i}: i \in I\right\}$ uma família de espaços topológicos. Se $d\left(X_{i}\right) \leq \mathfrak{m} \geq \omega$ para cada $i \in S$ e $|I| \leq 2^{\mathfrak{m}}$, então $d\left(\prod_{i \in I} X_{i}\right) \leq \mathfrak{m}$.

Demonstração. Vide [29].

Proposição 1.2.18. Para cada ordinal $\alpha \in \mathbb{O N}$, tem-se que $c\left(\{0,1\}^{\alpha}\right)=\omega$.

Demonstração. Seja $\left\{U_{t}: t \in T\right\}$ uma família de conjuntos abertos canônicos dois a dois disjuntos de $\{0,1\}^{\alpha}$ tal que $U_{t}=\prod_{\beta \in S_{t}} W_{\beta}^{t} \times\{0,1\}^{\alpha \backslash S_{t}}$ onde $S_{t} \in[\alpha]^{<\omega}$ e $W_{\beta}^{t}$ é um aberto próprio no espaço discreto $\{0,1\}$, para cada $t \in T$. Suponhamos por absurdo que $|T|>\omega$; passando talvez a um subconjunto, podemos supor também que $|T| \leq \mathfrak{c}$. Se $S_{0}=\bigcup_{t \in T} S_{t}$, observemos que $\left|S_{0}\right| \leq|T| \leq \mathfrak{c}$; pelo teorema 1.2.17, tem-se que o produto $\{0,1\}^{S_{0}}$ é separável. Notemos que os membros da família

$$
\mathscr{W}=\left\{\prod_{s \in S_{t}} W_{s}^{t} \times\{0,1\}^{S_{0} \backslash S_{t}}: t \in T\right\}
$$

são subconjuntos abertos não vazios de $\{0,1\}^{S_{0}}$ dois a dois disjuntos; de fato, para cada $t \in T$, temos $U_{t}=\prod_{s \in S_{t}} W_{s}^{t} \times\{0,1\}^{S_{0} \backslash S_{t}} \times\{0,1\}^{\alpha \backslash S_{0}}$; assim, $\prod_{s \in S_{t}} W_{s}^{t} \times\{0,1\}^{S_{0} \backslash S_{t}} \cap \prod_{s \in S_{r}} W_{s}^{r} \times\{0,1\}^{S_{0} \backslash S_{r}}=\varnothing$ sempre que $U_{t} \cap U_{r}=\varnothing$. Se $D \subseteq\{0,1\}^{S_{0}}$ fosse um subconjunto enumerável e denso em $\{0,1\}^{S_{0}}$, visto que cada membro de $\mathscr{W}$ contém um elemento de $D, \omega=|D| \geq|T|$, uma contradição. Em conseqüência, $c\left(\{0,1\}^{\alpha}\right)=\omega$ para qualquer $\alpha \in \mathbb{O N}$.

Proposição 1.2.19. Seja $(X, \tau)$ um espaço topológico $T_{2}$ compacto. Então, $w(X) \leq|X|$.

Teorema 1.2.20 (Teorema de Čech-Pospíšil). Se X é um espaço compacto tal que $\chi(p, X) \geq \kappa$ para cada $p \in X$, então $|X| \geq 2^{\kappa}$.

Demonstração. Vide [43].

\subsection{Grupos topológicos e estruturas relacionadas}

\section{Alguns conceitos algébricos}

Seja $(G, \cdot)$ um grupo. O símbolo $e_{G}$ será utilizado para denotar o elemento identidade de $G$, e, para cada $x \in G, x^{-1}$ representará o elemento inverso de $x$. Se tratando de um grupo abeliano $(G,+), 0_{G}$ denota seu elemento identidade e $-x$ é o elemento inverso de $x \in G$. 
Quando não houver risco à interpretação, representamos a identidade do grupo $G$ por 0 ou $e$, conforme o caso.

Um semigrupo é um conjunto não vazio $S$ munido de uma operação binaria associativa. Um elemento $e$ de um semigrupo $S$ é uma identidade de $S$ se $e x=x=x e$ para todo $x \in S$.

Se $S$ é um semigrupo, para um elemento fixo $a \in S$, as aplicações $x \mapsto a x$ e $x \mapsto x a$ são chamadas de ações a direita e esquerda de $x \operatorname{em} S$, e são denotadas por $\lambda_{a}$ e $\varrho_{a}$, respetivamente.

Se $G$ é um grupo, a aplicação Inv : $x \mapsto x^{-1}$ de $G$ em si mesmo é chamada de inversão. As ações a direita e esquerda de cada elemento $a \in G$ sobre $G$ são, neste caso, bijeções e são ditas translações de $G$ por $a$.

Definição 1.3.1. Um elemento $a$ de um grupo $G$ diz-se de ordem finita, ou, equivalentemente, elemento de torção se $a^{n}=e$ para algum $n \in \mathbb{N}$. Neste caso, o menor $n \in \mathbb{N}$ tal que $a^{n}=e$ é chamado a ordem de $a$ e denotado por $o(a)$. Se todos os elementos de $G$ são de ordem finita, dizemos então que $G$ é um grupo de torção. Se o grupo $G$ não possui elementos de ordem finita, exceto a identidade $e_{G}$, então $G$ é chamado livre de torção.

O conjunto de todos os elementos $a \in G$ de ordem finita, é chamado de parte de torção de $G$ e denotada por tor $(G)$. Se o grupo $G$ é Abeliano, tor $(G)$ constitui um subgrupo de $G$.

Definição 1.3.2. Um grupo $G$ é dito divisível se dados $n \in \mathbb{N} \backslash\{0\}, x \in G$ existe um elemento $y \in G$ tal que $y^{n}=x$.

Uma propriedade fundamental dos grupos abelianos divisíveis constitui o resultado a seguir:

Teorema 1.3.3. Sejam $G$ um grupo abeliano, $H$ um subgrupo de $G$ e $F$ um grupo abeliano divisível. Então para cada homomorfismo $f: H \rightarrow F$ existe um homomorfismo $\bar{f}: G \rightarrow F$ tal que $\bar{f} \uparrow_{H}=f$.

Demonstração. Vide 1.1.3 de [4].

Exemplo 1.3.4. Seja $\mathbb{T}=\{x \in \mathbb{C}:\|x\|=1\}$ a circunferência unitária no plano complexo. Naturalmente, $(\mathbb{T}, \times)$ é um subgrupo de $(\mathbb{C}, \times)$ onde $\times$ representa o produto usual de números complexos. Se $z \in \mathbb{T}$, existe $\theta \in[0,2 \pi)$ tal que $z=e^{i \theta}=\cos (\theta)+i \sin (\theta)$; pela fórmula de D’Moivre, para cada $n \in \mathbb{N} \backslash\{0\}$ existem exatamente $n$ raízes n-ésimas de $z$ determinadas pela igualdade

$$
y_{k}=e^{\frac{\theta+2 \pi i k}{n}}=\cos \left(\frac{\theta+2 \pi i k}{n}\right)+i \sin \left(\frac{\theta+2 \pi i k}{n}\right)
$$

onde $k \in\{0,1, \ldots, n-1\}$. Assim, $\mathbb{T}$ constitui um grupo abeliano divisível. 


\section{Grupos com topologia}

Definição 1.3.5. Seja $(G, \cdot)$ um grupo. Uma topologia $\tau$ em $G$ é chamada de topologia de grupo se as aplicações

$$
\begin{array}{rlrl}
G \times G & \rightarrow G & G & \rightarrow G \\
(x, y) & \mapsto x y & x & \mapsto x^{-1}
\end{array}
$$

tornam-se contínuas considerando o espaço $(G, \tau)$ e a topologia produto no espaço $G \times G$. Um grupo topológico é uma tripla $(G, \cdot, \tau)$ onde $G$ é um grupo e $\tau$ é uma topologia de grupo sobre $G$.

Definição 1.3.6. Seja $(S, \cdot)$ um semigrupo. A tripla $(S, \cdot, \tau)$ é um semigrupo topológico se $\tau$ é uma topologia definida no conjunto $S$ que torna contínua a aplicação

$$
\begin{aligned}
S \times S & \rightarrow S \\
(s, t) & \mapsto s t
\end{aligned}
$$

onde $S \times S$ é munido da topologia produto.

De acordo com as definições anteriores, a aplicação $x \mapsto x^{-1}$ definida num grupo topológico $G$ é um homeomorfismo. Se $V \subseteq G$, dizemos que $V$ é um subconjunto simétrico se $V^{-1}=V$. Note-se que para cada $U \subseteq G, U \cap U-1$ é simétrico; assim, como $U^{-1}$ é aberto para cada subconjunto aberto $U$ de $G$, concluímos que os subconjuntos simétricos abertos formam um sistema de vizinhanças básicas no ponto $e_{G} \in G$.

Também é fácil ver que se $G$ é um grupo topológico, para cada $a \in G$, as translações $\lambda_{a}, \varrho_{a}$ e os automorfismos internos $x \mapsto a x a^{-1}$ são homeomorfismos. Daí, se $U$ é um subconjunto aberto de $G$ então os conjuntos $a U$ e $U a$ são também abertos para cada $a \in G$. Um corolário imediato das afirmações anteriores é a homogeneidade dos grupos topológicos.

Proposição 1.3.7. Todo grupo topológico G é um espaço topológico homogêneo, isto é, para cada $x, y \in G$, existe um homeomorfismo $f: G \rightarrow G$ tal que $f(x)=y$.

Demonstração. Considere a translação $\lambda_{a}: G \rightarrow G$ onde $a=y x^{-1}$. Naturalmente, $\lambda_{a}$ é um homeomorfismo e $\lambda_{a}(x)=y$.

Naturalmente, todo grupo (semigrupo) é um grupo topológico considerando a topologia discreta ou a topologia caótica. Exemplos não triviais de grupos topológicos são listados adiante. 
Exemplo 1.3.8. Seja $\tau_{\text {Sor }}$ a topologia sobre $\mathbb{R}$ cuja base $\mathcal{B}$ consta dos intervalos semi-abertos $[a, b)=\{x \in \mathbb{R}: a \leq x<b\}$, onde $a, b \in \mathbb{R}$ e $a<b$. Observe que a aplicação $f: \mathbb{R} \times \mathbb{R} \rightarrow \mathbb{R}$ definida por $f(x, y)=x+y$ é contínua considerando a topologia $\tau_{\text {Sor }}$, isto é, $\left(\mathbb{R},+, \tau_{\text {Sor }}\right)$ é um semigrupo topológico. Pode ser fácilmente verificado que a aplicação $x \mapsto-x$ não é contínua, $\operatorname{logo} \tau_{\text {Sor }}$ não é uma topologia de grupo para $(\mathbb{R},+)$.

Exemplo 1.3.9. O grupo $(\mathbb{T}, \times)$ munido da topologia de subespaço induzida por $\mathbb{C}$ com a topologia usual é um grupo topológico.

Exemplo 1.3.10. O grupo das matrizes inversíveis de ordem $n$ com entradas reais, denotado por $G L(n, \mathbb{R})$, é um grupo topológico munido da topologia de subespaço induzida por $\mathbb{R}^{n^{2}}$. $G L(n, \mathbb{R})$ é chamado de grupo geral linear de grau $n$ sobre $\mathbb{R}$.

Exemplo 1.3.11. O produto topológico $\mathbb{T}^{\kappa}$ de $\kappa$ cópias do grupo do círculo $\mathbb{T}$ considerando a operação de soma em $\mathbb{T}$ coordenada a coordenada é um grupo topológico Hausdorff compacto. No contexto dos grupos topológicos, $\mathbb{T}^{\mathrm{K}}$ não faz o papel de espaço universal que o cubo $[0,1]^{\mathrm{K}}$ tem nos espaços de Tychonoff. De fato, não é verdadeiro que todo grupo compacto é topologicamente isomorfo a um subgrupo topológico de $\mathbb{T}^{\kappa}$ sendo esta asserção válida só quando o grupo em questão, além de compacto, é abeliano.

Os axiomas de separação nos grupos topológicos tem uma simplificação particularmente importante:

Proposição 1.3.12. Seja $(G, \cdot, \tau)$ um grupo topológico. Se $\overline{\left\{e_{G}\right\}}=\left\{e_{G}\right\}$, então $G$ é um espaço topológico regular.

Demonstração. Vide 1.2 de [63].

De acordo com a proposição anterior, um grupo topológico $T_{1}$ é também de Hausdorff. Assim, nos grupos topológicos, as propriedades de ser regular, Hausdorff e $T_{1}$ coincidem. De fato, é fácil mostrar que um grupo topológico satisfazendo o axioma de separação $T_{0}$ é Hausdorff e assim, regular. Mais ainda,

Proposição 1.3.13. Todo grupo topológico de Hausdorff é um espaço de Tychonoff.

Demonstração. Vide 2.4 de [63].

Entenderemos por subgrupo de um grupo topológico $G$ um subgrupo algébrico $H$ de $G$ munido da topologia de subespaço induzida por $G$. Note que o fecho $\bar{H}$ de $H$ em $G$ é também um subgrupo (topológico) de $G$. 
Seja $H$ um subgrupo normal e fechado de um grupo topológico $G$. Se $G / H=\{x H: x \in G\}$ é o grupo quociente, denotamos por $q: G \rightarrow G / H$ o homomorfismo natural $x \mapsto x H$; consideremos em $G / H$ a topologia final gerada por $q$, isto é, um subconjunto $V \subseteq G / H$ é aberto se e somente se $q^{-1}[V]$ é aberto em $G$. Pela definição, a aplicação $q$ é contínua e também é aberta; de fato, se $U \subseteq G$ é aberto, então $q^{-1} q(U)=U \cdot H$ é aberto em $G$, isto é, $V=q(U)$ é aberto em $G / H$. É fácil ver que o $G / H$ munido da topologia final gerada por $q$ é um grupo topológico. Note que a identidade $e_{G / H}=\pi\left(e_{G}\right)$ é fechada em $G / H$ pois $H=q^{-1}\left(e_{G / H}\right)$ é fechado em $G$. Assim, pela proposição 1.3.12, o grupo $G / H$ é de Hausdorff.

Exemplo 1.3.14. Seja $\mathbb{R}$ o grupo aditivo dos números reais munido da topologia usual e seja $\mathbb{Z}$ o subgrupo dos números inteiros. Claramente, $\mathbb{R}$ é um grupo topológico e $\mathbb{Z}$ um subgrupo fechado de $\mathbb{R}$. Como $\mathbb{R}$ é abeliano, $\mathbb{Z}$ é um subgrupo normal de $\mathbb{R}$. O grupo quociente $\mathbb{R} / \mathbb{Z}$ é compacto pois coincide com a imagem do intervalo fechado $[0,1]$ sob o homomorfismo quociente $q: \mathbb{R} \rightarrow \mathbb{R} / \mathbb{Z}$ dado por $r \mapsto r+\mathbb{Z}$.

Sejam $G$ e $H$ grupos topológicos e $f: G \rightarrow H$ um homomorfismo contínuo. Se $f$ é um isomorfismo algébrico além de ser homeomorfismo, então $f$ é dito um isomorfismo de grupos topológicos. Como todo grupo topológico é um espaço homogêneo, segue que um homomorfismo $f: G \rightarrow H$ é contínuo se e somente se é contínuo na identidade $e_{G}$.

Exemplo 1.3.15. Os grupos topológicos $(\mathbb{R} / \mathbb{Z},+)$ e $(\mathbb{T}, \times)$ são isomorfos. Com efeito, consideremos a aplicação

$$
\begin{aligned}
\psi: \mathbb{R} & \rightarrow \mathbb{T} \\
r & \mapsto e^{2 \pi i r}
\end{aligned}
$$

De acordo com as propriedades elementares do produto nos números complexos, $\psi$ é um homomorfismo contínuo entre os grupos $(\mathbb{R},+)$ e $(\mathbb{T}, \times)$. Note que $\psi(r)=0_{\mathbb{T}}=1$ se e somente se $r \in \mathbb{Z}$. Como $\psi$ é uma aplicação sobrejetora, pelos teoremas de isomorfismo em grupos algébricos temos que $\mathbb{R} / \operatorname{ker}(\psi)=\mathbb{R} / \mathbb{Z} \cong \mathbb{T}$ onde $f: \mathbb{R} / \mathbb{Z} \rightarrow \mathbb{T}$ definida como $f(r+\mathbb{Z})=\psi(r)$ testemunha o isomorfismo; além disso, $\psi=f \circ q$ onde $q: \mathbb{R} \rightarrow \mathbb{R} / \mathbb{Z}$ é como no exemplo 1.3.14. Como $\mathbb{R} / \mathbb{Z}$ está munido da topologia quociente e $\psi$ é contínuo segue que $f$ é contínuo; notemos que $\mathbb{R} / \mathbb{Z}$ é compacto e $\mathbb{T}$ é Hausdorff, logo, $f$ é também um homeomorfismo. Assim, $f$ é um isomorfismo de grupos topológicos. 


\section{CAPÍTULO 2}

\section{Grupos de van Douwen e MA}

Os grupos topológicos enumeravelmente compactos sem sequências não triviais convergentes apareceram de maneira implícita com a finalidade de resolver um outro problema: a preservação sob produtos da compacidade enumerável em grupos topológicos.

Como é bem sabido, a compacidade na classe dos grupos topológicos compactos é fechada sob produtos, subgrupos fechados e preservada por homomorfismos contínuos; por outra parte, um célebre teorema de Comfort e Ross [21] afirma que a pseudocompacidade constitui uma propriedade produtiva na classe dos grupos topológicos , é preservada por homomorfismos contínuos mas, nem sempre um subgrupo fechado de um grupo pseudocompacto é pseudocompacto. De fato, qualquer grupo totalmente limitado é topologicamente isomorfo a um subgrupo fechado de um grupo pseudocompacto. Pelo contrário, a compacidade enumerável em grupos topológicos é uma propriedade hereditária para subgrupos fechados, preservada sob homomorfismos contínuos mas ZFC não decide se a compacidade enumerável é ou não uma propriedade produtiva na classe dos grupos topológicos. De fato, em [17] aparece a seguinte questão que ainda permanece em aberto:

Questão 2.1 (W.W. Comfort). É um teorema de ZFC que existem dois grupos topológicos enumeravelmente compactos cujo produto não é enumeravelmente compacto?

E. van Douwen em [26] foi quem mostrou o primeiro exemplo relativo à questão 2.1, porém, assumindo MA. De fato, sob MA van Douwen constrói um subgrupo $G \subseteq 2^{\text {c }}$ enumeravelmente compacto sem sequências não triviais convergentes; posteriormente, em ZFC, van Douwen define dois subgrupos enumeravelmente compactos $H_{1}, H_{2} \subseteq G$ tais que $\left|H_{1} \cap H_{2}\right|=\omega$. Portanto, $\left\{(x, x): x \in H_{1} \cap H_{2}\right\}$ constitui um subgrupo fechado, discreto e infinito no produto $H_{1} \times H_{2}$, logo o grupo $H_{1} \times H_{2}$ não é enumeravelmente compacto. 
Em virtude das considerações acima, um grupo topológico $G$ infinito, abeliano, enumeravelmente compacto e sem seqüências não triviais convergentes é chamado de grupo de van Douwen.

Em 1991, Hart e van Mill [40] melhoraram o resultado de van Douwen, obtendo sob $\mathrm{MA}_{\text {enumerável }}$ um grupo enumeravelmente compacto $H$ de cardinalidade $\mathfrak{c}$ cujo quadrado $H^{2}$ não é enumeravelmente compacto. O exemplo destes autores é um subgrupo $H=C \oplus K$ do cubo de Cantor $\{0,1\}^{c}$ onde $C$ é um subgrupo enumerável e $K$ é um grupo no qual o fecho de qualquer subconjunto $A \in[K]^{\omega}$ é compacto. Em particular, $H$ contém infinitos subgrupos compactos e assim, sequências não triviais convergentes. Daí, MA permaneceu como a hipótese mais fraca para obter um grupo enumeravelmente compacto sem seqüências não triviais convergentes.

Em 1976, assumindo CH, Hajnal e Juhász [39] construíram um subgrupo $G$ de $\{0,1\}^{\mathrm{c}}$ de cardinalidade $\mathfrak{c}$, enumeravelmente compacto, hereditariamente separável e sem sequências não triviais convergentes. Este exemplo, junto com o argumento feito em ZFC do exemplo de van Douwen, também implica a existência de dois grupos enumeravelmente compactos cujo produto não é enumeravelmente compacto.

Todos os grupos mencionados até agora são subgrupos de $\{0,1\}^{\mathrm{c}}$ de maneira que constituem grupos booleanos e zero-dimensionais. Tkačenko, no compendio de problemas [17], perguntou se existe em ZFC uma topologia de grupo enumeravelmente compacta compatível sobre o grupo Abeliano livre com c geradores, obtendo posteriormente uma tal topologia sob CH [62]. A construção de Tkačenko baseia-se nas idéias de Hajnal e Juhász em [39], ou seja, $\mathrm{CH}$ é usado para construir um subgrupo denso $G \subseteq \mathbb{T}^{c}$ enumeravelmente compacto que é hereditariamente finalmente denso (HFD), isto é, para qualquer subconjunto infinito $A$ de $G$, existe $\alpha<\mathfrak{c}$ tal que o conjunto de restrições $\left\{s \uparrow_{\mathfrak{a} a}: s \in A\right\}$ é denso no segmento final $\mathbb{T}^{\mathrm{\wedge} \backslash \alpha}$, e, naturalmente, algebricamente isomorfo ao grupo livre com um conjunto gerador de cardinalidade c. Sendo HFD, $G$ não contém sequências não triviais convergentes.

Este capítulo irá tratar da existência de grupos enumeravelmente compactos sem sequên-

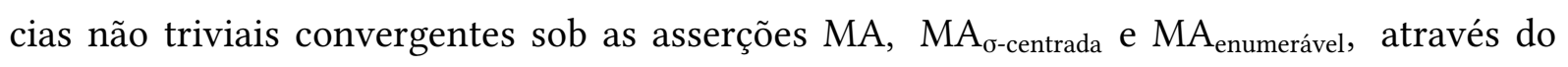
estudo das construções feitas por van Douwen, Tomita e Koszmider et. al., respetivamente. Todos os espaços considerados no decorrer do capítulo são de Hausdorff. 


\subsection{Primeira construção de E. van Douwen a partir de MA}

Como referimos anteriormente, o contraexemplo de van Douwen constitui um grupo booleano, isto é, para cada $x \in G, x+x=0_{G}$. Antes de começar com os detalhes da construção, resulta conveniente estudar alguns fatos sobre este tipo simples, mas especial de grupos.

\section{Grupos booleanos}

Se $G$ é um grupo booleano, a solução da equação $a+x=b$ é $x=a+b$. Note que $(a+b)+(b+a)=0$ implica que $a+b=b+a$, ou seja, $G$ é abeliano; daí, se $A, B$ são subgrupos de $G$, então $\langle A \cup B\rangle=A+B$.

Nos grupos booleanos, estender homomorfismos resulta ser particularmente simples. Os resultados a seguir mostram este fato.

Teorema 2.1.1. Sejam $G$ e $H$ grupos booleanos, e sejam $A$ e $B$ subgrupos de $G$. Suponha que $p: A \rightarrow H$ e $q: B \rightarrow H$ são homomorfismos. Se $p \uparrow_{A \cap B}=q \uparrow_{A \cap B}$, então existe um homomorfismo $r: A+B \rightarrow H$ que estende os homomorfismos $p$ e $q$.

Demonstração. Para cada $a \in A, b \in B$, defina $r(a+b)=p(a)+q(b)$. Afirmamos que a relação $r: A+B \rightarrow H$ é um homomorfismo de grupos. Para isto, basta ver que $r$ está bem definido, pois $p$ e $q$ são homomorfismos parciais. Suponha então que $a, c \in A, b, d \in B$ são tais que $a+b=c+d$. Observe que $a+c=b+d \in A \cap B$; daí, $p(a+c)=q(b+d)$, isto é, $p(a)+p(c)=q(b)+q(d)$. Dado que $H$ é booleano, $p(a)+q(b)=p(c)+q(d)$, ou seja $r(a+b)=r(c+d)$; portanto, $r$ está bem definido.

Corolário 2.1.2. Sejam $G$ e $H$ grupos booleanos. Se $A \subset G$ é subgrupo e $q: A \rightarrow H$ é um homomorfismo então para cada $y \in G \backslash A$ e $b \in H$ existe um homomorfismo $p:\{0, y\}+A \rightarrow H$ que estende q e satisfaz $p(y)=b$.

Demonstração. Considere o subgrupo $B=\{0, y\} \subseteq G$; defina $r: B \rightarrow H$ por $r(y)=b$, $r(0)=0_{H}$. Naturalmente, $r \in \operatorname{hom}(B, H)$ e $A \cap B=\{0\}$. Pelo teorema anterior, existe um homomorfismo $q: A+B=A+\{0, y\} \rightarrow H$ estendendo $q$ e $r$, em particular, satisfaz $p(y)=r(y)=b$.

\section{A construção indutiva}

E. van Douwen, construiu o grupo $G$ através de um processo indutivo sobre $\mathfrak{c}$, definindo em cada etapa $\omega \leq \alpha \leq \mathfrak{c}$ um subgrupo $G_{\alpha}$ de $2^{\alpha}$ e, finalmente tomando $G:=G_{\text {c }}$.

O seguinte lema, constitui o coração da prova: 
Lema 2.1.3. Assuma MA. Sejam $H$ um grupo Booleano, $I \in[H]^{\omega}$ e $\mathcal{K} \subseteq[H]^{\omega}$. Se $|H|<\mathfrak{c} e$ $|\mathcal{K}|<\mathfrak{c}$, então existe um homomorfismo $h: H \rightarrow 2$ tal que

a. $\left|I \cap h^{-1}[i]\right|=\omega$ para $i \in\{0,1\}$;

b. $K \cap h^{-1}[0] \neq \varnothing$ para cada $K \in \mathcal{K}$.

Demonstração. Seja J uma coleção infinita de subconjuntos infinitos de $I$ dois a dois disjuntos, e considere $\mathcal{L}=\mathcal{J} \cap \mathcal{K}$. É suficiente achar um homomorfismo $h: H \rightarrow 2$ tal que

$$
\text { para cada } L \in \mathcal{L} \text { e } i \in\{0,1\}, L \cap h^{-1}[i] \neq \varnothing
$$

Considere o conjunto

$$
\mathbb{P}=\{p: \text { existe um subgrupo finito } A \subseteq H \text { tal que } p \in \operatorname{hom}(A, 2)\}
$$

Se $p, q \in \mathbb{P}$, dizemos que $p \leq q$ se e somente se $q \subseteq p$. Naturalmente, se $\mathbb{G} \subseteq \mathbb{P}$ é um filtro, então $S=\bigcup_{p \in \mathbb{G}} \operatorname{dom}(p)$ é um subgrupo de $H$ e $\cup \mathbb{G} \in \operatorname{hom}(S, 2)$. De fato, dados $a, b \in S$ tome $p, q \in \mathbb{G}$ tais que $a \in \operatorname{dom}(p), b \in \operatorname{dom}(q)$; dado que $\mathbb{G}$ é filtro, existe $r \in \mathbb{G}$ tal que $r \leq p, q$. De acordo com a definição, $a, b \in \operatorname{dom}(r)$ de onde $a-b \in \operatorname{dom}(r) \subseteq S$, pois $\operatorname{dom}(r)$ é um subgrupo de $H$; portanto, $S$ é subgrupo. É obvio que $\bigcup \mathbb{G}$ é um homomorfismo.

Definamos agora alguns subconjuntos de $\mathbb{P}$, os quais constituem subconjuntos densos nessa ordem. Primeiro, para cada $x \in H$, seja $D_{x}=\{p \in \mathbb{P}: x \in \operatorname{dom}(p)\}$ e defina-se $\mathcal{D}=\left\{D_{x}: x \in H\right\}$.

Agora, para cada $L \in \mathcal{L}$ e $i \in 2$, seja

$$
E_{L, i}=\{p \in \mathbb{P}: \text { existe } y \in L \cap \operatorname{dom}(p) \text { tal que } p(y)=i\}
$$

e consideremos $\mathcal{E}=\left\{E_{L, i}: L \in \mathcal{L}, i \in 2\right\}$.

- Cada membro de $\mathcal{D}$ é denso: Seja $q \in \mathbb{P}$ e $y \in H$. Podemos assumir que $y \notin \operatorname{dom}(q)$; pelo corolário 2.1.2 existe um homomorfismo $p:\{0, y\}+\operatorname{dom}(q) \rightarrow 2$ que estende $q$, ou seja, $D_{y}$ é denso.

- Cada membro de $\mathcal{E}$ é denso: Sejam $q \in \mathbb{P}, L \in \mathcal{L}$ e $i \in 2$. Temos que achar $p \in \mathbb{P}$ satisfazendo $p \leq q$ e tal que $p(y)=i$ para algum $y \in L \cap \operatorname{dom}(p)$. Como $L$ é infinito, podemos escolher $y \in L \backslash \operatorname{dom}(q)$; pelo corolário 3.1.1. existe um homomorfismo $p:\{0, y\}+\operatorname{dom}(q) \rightarrow 2$ estendendo $q$ e tal que $p(y)=i, \log o, E_{L, i}$ é denso em $\mathbb{P}$. 
Finalmente, mostremos que $\mathbb{P}$ é uma ordem c.c.c. Seja $Q \subseteq \mathbb{P}$ não enumerável. Devemos achar $p, q \in Q$ elementos distintos e compatíveis. Para cada $A \in[H]^{<\omega}, 2^{A}$ é finito; daí, se $\{\operatorname{dom}(p): p \in Q\}$ fosse enumerável, $Q$ seria necessariamente enumerável, contradizendo a hipótese. Portanto, $|\{\operatorname{dom}(p): p \in Q\}|>\omega$. Em virtude do lema do $\Delta$-sistema (lema 1.1.46) existem uma coleção $Q^{\prime} \subseteq Q$ e $B \in[H]^{<\omega}$ satisfazendo $\left|Q^{\prime}\right|=|Q|$ e $\operatorname{dom}(p) \cap \operatorname{dom}(q)=B$ para quaisquer $p, q \in Q^{\prime}$ distintos. Como existe só um número finito de funções $f: B \rightarrow 2$, existem $p, q \in Q^{\prime}$ distintos que coincidem sobre $B$, isto é, $p \uparrow_{B}=q \uparrow_{B}$. Pelo lema 3.1., existe um homomorfismo $r: \operatorname{dom}(p)+\operatorname{dom}(q) \rightarrow 2$ estendendo $p$ e $q$; note que $r \in \mathbb{P}, \operatorname{logo} p, q \in Q^{\prime} \subseteq Q$ são compatíveis, isto é, $Q$ não é uma anticadeia.

Observe-se que $|\mathcal{D} \cup \mathcal{E}| \leq|\mathcal{D}|+|\mathcal{E}|=|H|+2|\mathcal{K}|<\mathfrak{c}$. Como $\mathbb{P}$ é c.c.c. ao estarmos assumindo MA, existe um filtro $\mathbb{G}$ que interseta a todo membro da família $\mathcal{D} \cup \mathcal{E}$. Observe que para todo $x \in H, \mathbb{G} \cap D_{x} \neq \varnothing$, logo $H=\bigcup_{p \in \mathbb{G}} \operatorname{dom}(p)$; também, para todo $L \in \mathcal{L}$ e $i \in 2, \mathbb{G} \cap E_{L, i} \neq \varnothing, \operatorname{logo}$ existe $y \in L$ satisfazendo $\bigcup \mathbb{G}(y)=i$, com o qual o homomorfismo $h:=\bigcup \mathbb{G}: H \rightarrow 2$ satisfaz $(*)$.

Com este lema, procedemos agora à construção indutiva:

Teorema 2.1.4. Sob MA, existe um grupo $G \subseteq 2^{c}$ enumeravelmente compacto e sem sequências não triviais convergentes.

Demonstração. Recursivamente, definimos uma coleção de ordinais $\left\{\sigma_{\alpha}: \alpha<\mathfrak{c}\right\}$ :

$$
\sigma_{\alpha}= \begin{cases}\omega, & \text { se } \alpha=\omega \\ \sigma_{\beta}+\left|\sigma_{\beta}\right|, & \text { se } \alpha=\beta+1 \\ \sup _{\omega \leq \lambda<\alpha} \sigma_{\lambda}, & \text { se } \alpha \text { é ordinal limite }\end{cases}
$$

Note que se $\alpha<\mathfrak{c}, \sigma_{\alpha}<\mathfrak{c}$. Com efeito, tome $\kappa<\mathfrak{c}$ regular tal que $\alpha<\kappa$; dado que $\left(\sigma_{\beta}\right)_{\beta<\alpha}$ constitui uma seqüência crescente de comprimento menor que $\kappa$, necessariamente $\sigma_{\alpha}<\kappa$ para todo $\alpha<\kappa$. Obviamente, tomamos $\sigma_{\mathrm{c}}=\mathfrak{c}$.

Dado que $\sigma_{\mathfrak{c}}=\mathfrak{c}=\bigcup_{\alpha<\mathfrak{c}} \sigma_{\alpha}$, podemos tomar uma enumeração $\left\{I_{\xi}: \omega \leq \xi<\mathfrak{c}\right\}$ do conjunto $[\mathfrak{c}]^{\omega}$ tal que para cada $\omega \leq \xi<\mathfrak{c}, I_{\xi} \subseteq \sigma_{\xi}$.

De acordo com o anterior, para cada $\omega \leq \alpha<\mathfrak{c}$, será definido o subgrupo $G_{\alpha}=\left\{x_{\alpha, \xi}: \xi<\right.$ $\left.\sigma_{a}\right\}$ de modo que as seguintes condições sejam satisfeitas:

1. se $\xi<\eta<\sigma_{\alpha}$, então $x_{\alpha, \xi} \neq x_{\alpha, \eta}$ onde $\omega \leq \alpha \leq \sigma_{\alpha}$;

2. se $\xi<\sigma_{\alpha}$, então $\alpha<\beta$ implica $x_{\alpha, \xi} \subseteq x_{\beta, \xi}$; 
3. se $\xi, \eta, \zeta<\sigma_{\alpha}$ são tais que $x_{\alpha, \xi}+x_{\alpha, \eta}=x_{\alpha, \zeta}$ então para $\beta>\alpha$ tem-se $x_{\beta, \xi}+x_{\beta, \eta}=x_{\beta, \zeta}$;

4. se $\omega \leq \xi<\alpha<\mathfrak{c}$, então existe $\lambda_{\xi}<\sigma_{\xi+1}$ tal que $x_{\alpha, \lambda \xi}$ é um ponto de acumulação da seqüência em $2^{\alpha}$ determinada por $I_{\xi}$, i.e., a seqüência $\left\{x_{\alpha, \eta}: \eta \in I_{\xi}\right\}$;

5. se $\omega \leq \xi<\mathfrak{c}$, então $\left|\left\{\eta \in I_{\xi}: x_{\xi+1, \eta}(\xi)=i\right\}\right|=\omega$ para $i=0$ e $i=1$.

Começamos a construção definindo as aproximações parciais do grupo $G$, isto é, os $G_{\alpha}$ por indução em c. Primeiro, seja $G_{\omega}$ um subgrupo infinito enumerável de $2^{\omega}$, enumerado por $\left\{x_{\omega, n}: n<\omega\right\}$ de forma que $x_{\omega, n} \neq x_{\omega, m}$ para cada $n \neq m$ em $\omega$. Observe-se que neste caso, as hipóteses de indução são trivialmente satisfeitas.

Seja $\omega<\alpha \leq$ c. Suponha que $G_{\beta}$ tem sido definido para cada $\beta<\alpha$, de forma tal que $G_{\beta}$ satisfaz as condições 1)-5). Consideramos dois casos:

i. $\alpha$ é ordinal limite. Como $\sigma_{\alpha}=\sup _{\omega<\beta<\alpha} \sigma_{\beta}$, para cada $\xi<\sigma_{\alpha}$ existe $\beta<\alpha$ de forma que $\xi<\sigma_{\beta}$; daí, a condição 2) força a definir para cada $\xi<\sigma_{\alpha}$

$$
x_{\alpha, \xi}=\bigcup\left\{x_{\beta, \xi}: \omega \leq \beta<\alpha \mathrm{e} \xi<\sigma_{\beta}\right\}
$$

Vejamos que definido dessa maneira, $G_{\alpha}:=\left\{\alpha_{\xi}: \xi \in \sigma_{\alpha}\right\}$ satisfaz as condições 1)-4).

a. Suponha que $\xi<\eta<\sigma_{\alpha}$; tome $\beta<\alpha$ de modo que $\eta<\sigma_{\beta}$. Pela hipótese de indução, $x_{\beta, \xi} \neq x_{\beta, \eta} \in 2^{\beta}$; daí, existe $t<\beta$ de modo que $x_{\beta, \xi}(t) \neq x_{\beta, \eta}(t)$, logo, pela construção, $x_{\alpha, \xi}(t)=x_{\beta, \eta}(t)=x_{\alpha, \eta}(t)$. Daí, $x_{\alpha, \xi} \neq x_{\alpha, \eta}$.

b. Se $\omega \leq \beta<\alpha$ e $\xi<\sigma_{\beta}$, pela construção é obvio que $x_{\beta, \xi} \subset x_{\alpha, \eta}$.

c. Suponha que $\gamma \leq \beta<\alpha$ e $\xi, \eta, \zeta<\sigma_{\beta}$ são tais que $x_{\beta, \xi}+x_{\beta, \eta}=x_{\beta, \zeta}$. Seja $t<\alpha$. Temos que mostrar que $x_{\alpha, \xi}(t)+x_{\alpha, \eta}(t)=x_{\alpha, \zeta}(t)$. Note que $x_{\beta, \theta}=x_{\alpha, \theta} \uparrow_{\beta}$, logo basta provar o caso em que $\alpha>t \geq \beta$. Como $\alpha$ é limite, podemos tomar $t>\theta \geq \beta$; em virtude da hipótese de indução $x_{\theta, \xi}+x_{\theta, \eta}=x_{\theta, \zeta}, \operatorname{logo}$

$$
x_{\theta, \xi}(t)+x_{\theta, \eta}(t)=x_{\theta, \zeta}(t)
$$

Pela definição dos $x_{\alpha, v}$ temos $x_{\alpha, \xi}(t)+x_{\alpha, \eta}(t)=x_{\alpha, \zeta}(t)$.

d. Vejamos agora que $x_{\alpha, \lambda \xi}$ é ponto de acumulação da seqüência $\left\{x_{\alpha, \eta}: \eta \in I_{\xi}\right\}$. Suponha que $\omega \leq \xi<\alpha$. Vamos mostrar que qualquer vizinhança de $x_{\alpha, \lambda_{\xi}} \in 2^{\alpha}$ contém infinitos termos da seqüência. Tome $L \in[\alpha]^{<\omega}$ não vazio. Temos que achar $\eta \in I_{\xi}$ de forma que 
$x_{\alpha, \eta} \neq x_{\alpha, \lambda_{\xi}}$ e $x_{\alpha, \lambda_{\xi}} \Gamma_{L} x_{\alpha, \eta}$. Note que, dado que $\alpha$ é limite, existe $\gamma$ satisfazendo $\omega \leq \gamma<\alpha$ tal que $L \subseteq \gamma$ e $\xi<\sigma_{\gamma}$.

Pela hipótese de indução, note que $x_{\gamma, \lambda_{\xi}}$ é ponto de acumulação de $\left\{x_{\gamma, \eta}: \eta \in I_{\xi}\right\} \subseteq 2^{\gamma}$.

Daí, existe $\eta \in I_{\xi}$ tal que $x_{\gamma, \eta} \neq x_{\gamma, \lambda_{\xi}} \subset x_{\alpha, \lambda_{\xi}}$ e $x_{\gamma_{\xi}} \uparrow_{L} \subset x_{\gamma, \eta}$.

Como $x_{\alpha, \lambda_{\xi}} \supset x_{\gamma, \lambda_{\xi}}$ observe então que $x_{\alpha, \eta} \neq x_{\alpha, \lambda_{\xi}}$ e $x_{\gamma, \lambda_{\xi}} \Gamma_{L}=x_{\alpha, \lambda_{\xi}} \Gamma_{L} \subset x_{\gamma, \eta} \subset x_{\alpha, \eta}$.

ii. $\alpha$ é um ordinal sucessor. Suponha que $\alpha=\gamma+1$. Pela condição 1), o conjunto $X:=$ $\left\{x_{\gamma, \xi}: \xi \in I_{\gamma}\right\}$ é infinito; assim, existe $p \in 2^{\gamma}$ tal que $p \in X^{\prime}$ posto que $2^{\gamma}$ é compacto,. Escolha $p^{\prime} \in 2^{\gamma} \backslash G_{\gamma}$ e tome $H=\left\{0, p^{\prime}\right\}+G_{\gamma}$. Note que $G_{\gamma} \cap\left(p^{\prime}+G_{\gamma}\right)=\varnothing$; dado que $G_{\gamma}=\left\{x_{\gamma, \eta}: \eta<\sigma_{\gamma}\right\}$, podemos enumerar a $H \backslash G_{\gamma}$ por meio de $\left\{x_{\gamma, \xi}: \sigma_{\gamma} \leq \xi<\sigma_{\gamma+1}\right\}$ onde $\lambda_{\gamma}$ é tal que $p=x_{\gamma, \lambda_{\gamma}}$. De acordo com a condição 4), para $\omega \leq \xi \leq \gamma, x_{\gamma, \lambda_{\xi}}$ é ponto de acumulação de $\left\{x_{\gamma, \eta}: \eta \in I_{\xi}\right\}$.

A idéia é achar uma função $h: H \rightarrow 2$ que nos permitirá definir, de maneira conveniente, para cada função em $H$ a coordenada $\gamma$-ésima. Requere-se que $h$ satisfaça:

a. $h$ é homomorfismo;

b. se $\omega \leq \xi \leq \gamma$, então $x_{\gamma, \lambda_{\xi}}$ é ponto de acumulação de $\left\{x_{\gamma, \eta}: \eta \in I_{\xi}\right.$ e $\left.h\left(x_{\gamma, \eta}\right)=h\left(x_{\gamma, \lambda_{\xi}}\right)\right\}$;

c. $\left|\left\{\eta \in I_{\gamma}: h\left(x_{\gamma, \eta}\right)=i\right\}\right|=\omega$ para $i=0$ e $i=1$.

Resulta conveniente achar um equivalente à condição b). Para isso, se $\xi \leq \gamma$, definimos

$$
K_{\xi}=\left\{\left\{x_{\gamma, \eta}: \eta \in I_{\xi}, x_{\gamma, \eta} \neq x_{\gamma, \lambda_{\xi}} \text { e } x_{\gamma, \lambda_{\xi}} \uparrow_{L} \subseteq x_{\gamma, \eta}\right\}: L \in[\gamma]^{<\omega}\right\}
$$

Seja $\mathcal{K}=\bigcup\left\{\left\{x_{\gamma, \lambda}+K: K \in K_{\xi}\right\}: \omega \leq \xi \leq \gamma\right\}$. Vejamos que b) é equivalente à condição

b'. para todo $M \in \mathcal{K}$, existe $x \in M$ tal que $h(x)=0$.

$\diamond$ b) $\Rightarrow$ b') Seja $M \in \mathcal{K}$. Então $M=x_{\gamma, \lambda_{\xi}}+K$ para algum $K \in K_{\xi}$; pela definição de $K_{\xi}$, existe $L \in[\gamma]^{<\omega}$ de modo que $K=\left\{x_{\gamma, \eta}: \eta \in I_{\xi}, x_{\gamma, \eta} \neq x_{\gamma, \lambda_{\xi}}\right.$ e $\left.x_{\gamma, \lambda_{\xi}} \uparrow_{L} \subset x_{\gamma, \eta}\right\}$. Pela condição b), $x_{\gamma, \lambda_{\xi}}$ é ponto de acumulação de $S_{\xi}=\left\{x_{\gamma, \eta}: \eta \in I_{\xi}\right.$ e $\left.h\left(x_{\gamma, \eta}\right)=h\left(x_{\gamma, \lambda_{\xi}}\right)\right\}$; considere o aberto básico de $x_{\gamma, \lambda_{\xi}}$ dado por $U_{L}=\left\{x \in 2^{\gamma}: x_{\gamma, \lambda_{\xi}} \Gamma_{L} \subseteq x\right\}$. Então, existe $\eta \in I_{\xi}$ tal que $x_{\gamma, \eta} \in S_{\xi} \cap U_{L}$, onde $\eta \neq \lambda_{\xi}$. Note que $x_{\gamma, \eta} \in K \in K_{\xi}, \operatorname{logo} x:=x_{\gamma, \lambda_{\xi}}+x_{\gamma, \eta} \in M$ e

$$
h(x)=h\left(x_{\gamma, \lambda}+x_{\gamma, \eta}\right)=h\left(x_{\gamma, \lambda \xi}\right)+h\left(x_{\gamma, \eta}\right)=h\left(x_{\gamma, \eta}\right)+h\left(x_{\gamma, \eta}\right)=0
$$

$\left.\diamond \mathrm{b}^{\prime}\right) \Rightarrow$ b) Seja $L \in[\gamma]^{\omega}$ e sejam $S_{\xi}$ e $U_{L}$ como acima. Seja $A_{L}=\left\{x_{\gamma, \eta}: \eta \in I_{\xi}\right\} \cap U_{L} \backslash\left\{x_{\gamma, \lambda_{\xi}}\right\}$. Note que $A_{L} \in K_{\xi}$. Considere agora $x_{\gamma, \lambda_{\xi}}+A_{L} \in \mathcal{K}$. Pela condição b'), existe $x \in x_{\gamma, \lambda_{\xi}}+A_{L}$ 
tal que $h(x)=0$, ou seja, existe $\eta \in I_{\xi}$ tal que $h\left(x_{\gamma, \lambda_{\xi}}+x_{\gamma, \eta}\right)=h\left(x_{\gamma, \lambda_{\xi}}\right)+h\left(x_{\gamma, \eta}\right)=0$, isto é, $h\left(x_{\gamma, \lambda_{\xi}}\right)=h\left(x_{\gamma, \eta}\right)$; note que $x_{\gamma, \eta} \in S_{\xi} \cap U_{L} \backslash\left\{x_{\gamma, \lambda_{\xi}}\right\}$, logo $x_{\gamma, \lambda_{\xi}}$ é ponto de acumulação de $S_{\xi}$.

Observemos que pela definição de $x_{\gamma, \lambda}$, para cada $L \in[\gamma]^{\omega}$ o conjunto $\left\{x_{\gamma, \eta}: \eta \in I_{\xi}, x_{\gamma, \eta} \neq\right.$ $x_{\lambda_{\xi}}$ e $\left.x_{\gamma, \lambda_{\xi}} \uparrow_{L} \subseteq x_{\gamma, \eta}\right\}$ é infinito. Daí, cada membro de $\mathcal{K}$ é infinito também.

Como $x_{\gamma, \lambda_{\xi}}$ é ponto de acumulação de $\left\{x_{\gamma, \eta}: \eta \in I_{\xi}\right\}$, podemos aplicar o lema 2.1.3 considerando o subconjunto infinito de $H$ dado por $I:=\left\{x_{\gamma, \eta}: \eta \in I_{\gamma}\right\}$ e a coleção

$$
\mathcal{K}=\bigcup\left\{\left\{x_{\gamma, \lambda_{\xi}}+K: K \in K_{\xi}\right\}: \omega \leq \xi \leq \gamma\right\}
$$

cuja cardinalidade é $|\gamma|<\mathfrak{c}$.

Daí, obtém-se um homomorfismo $h: H \rightarrow 2$ tal que:

$\diamond$ para cada $i \in 2, \mid\left\{x_{\gamma, \eta}: \eta \in I_{\gamma}\right.$ e $\left.h\left(x_{\gamma, \eta}\right)=i\right\} \mid=\omega$, e,

$\diamond$ para todo $K \in \mathcal{K}$, existe $x \in K$ tal que $h(x)=0$

isto é, $h$ satisfaz as condições a), b) e c). Definimos então, $G_{\gamma+1}=\left\{x^{\prec} h(x): x \in H\right\}$. Vejamos que $G_{\gamma+1}$ satisfaz as condições 1)-5).

Sabemos que $G_{\gamma} \subset H$, logo para $\xi<\sigma_{\gamma}$, tem-se $x_{\gamma+1, \xi}=x_{\gamma, \xi} h\left(x_{\gamma, \xi}\right)$. Se $\sigma_{\gamma} \leq \xi<\sigma_{\gamma+1}$, tome $x \in H \backslash G_{\gamma}$ tal que $x=x_{\gamma, \xi}$. Neste caso, $x_{\gamma+1, \xi}=x_{\gamma, \xi} h\left(x_{\gamma, \xi}\right)$. Pela construção, é obvio que $G_{\gamma+1}$ satisfaz 1) e 2). Agora, se $\xi, \eta, \zeta<\sigma_{\gamma}$ são tais que $x_{\gamma, \xi}+x_{\gamma, \eta}=x_{\gamma, \zeta}$ temos

$$
\begin{aligned}
\left(x_{\gamma+1, \xi}+x_{\gamma+1, \eta}\right)(\gamma) & =x_{\gamma+1, \xi}(\gamma)+x_{\gamma+1, \eta}(\gamma) \\
& =h\left(x_{\gamma, \xi}\right)+h\left(x_{\gamma, \eta}\right) \\
& =h\left(x_{\gamma, \xi}+x_{\gamma, \eta}\right) \\
& =h\left(x_{\gamma, \xi}\right) \\
& =x_{\gamma+1, \xi}(\gamma)
\end{aligned}
$$

Como $x_{\alpha, \theta} \subseteq x_{\gamma+1, \theta}$ para todo $\alpha \leq \gamma$, isto é suficiente para mostrar que $x_{\gamma+1, \eta}+x_{\gamma+1, \xi}=x_{\gamma+1, \xi}$, obtendo assim a condição 3).

Seja $\omega \leq \xi \leq \gamma$. Vamos ver que $x_{\gamma+1, \lambda_{\xi}}$ é ponto de acumulação da seqüência $\left\{x_{\gamma+1, \eta}: \eta \in I_{\xi}\right\}$. Tome $L \in[\gamma+1]^{<\omega}$ não vazio; se $\gamma \notin L$, dado que $x_{\gamma, \eta} \subseteq x_{\gamma+1, \eta}$ para todo $\eta$, pela hipótese de indução sabemos que $x_{\gamma, \lambda_{\xi}}$ é ponto de acumulação de $\left\{x_{\gamma, \eta}: \eta \in I_{\xi}\right\}$, logo o conjunto $\left\{x_{\gamma, \eta}: \eta \in I_{\xi}, x_{\gamma, \eta} \neq x_{\gamma, \lambda_{\xi}}\right.$ e $\left.x_{\gamma, \lambda_{\xi}} \Gamma_{L} \subseteq x_{\gamma, \eta}\right\}$ é infinito; como para cada $\eta$ temos $x_{\gamma+1, \eta}=x_{\gamma, \eta}^{-} h\left(x_{\gamma, \eta}\right)$, 
o conjunto

$$
\left\{x_{\gamma, \eta}: \eta \in I_{\xi}, x_{\gamma+1, \eta} \neq x_{\gamma+1, \lambda_{\xi}} \text { e } x_{\gamma+1, \lambda_{\xi}} \uparrow_{L} \subseteq x_{\gamma+1, \eta}\right\}
$$

é infinito.

Suponha agora que $\gamma \in L$; pela condição b) sabemos que $x_{\gamma, \lambda}$ é ponto de acumulação do conjunto $\left\{x_{\gamma, \eta}: \gamma \in I_{\xi}, h\left(x_{\gamma, \eta}\right)=h\left(x_{\gamma, \lambda_{\xi}}\right)\right\}$. De acordo com a definição dos $x_{\gamma+1, \eta}$, o conjunto $\left\{x_{\gamma+1, \eta}: \eta \in I_{\xi}, x_{\gamma+1, \eta} \neq x_{\gamma+1, \lambda_{\xi}}\right.$ e $\left.x_{\gamma+1, \lambda_{\xi}} \uparrow_{L} \subseteq x_{\gamma+1, \eta}\right\}$ também é infinito; portanto, $x_{\gamma+1, \lambda_{\xi}}$ é ponto de acumulação de $\left\{x_{\gamma+1, \eta}: \eta \in I_{\xi}\right\}$, ou seja, $G_{\gamma+1}$ satisfaz a condição 4).

Agora, considere o conjunto $\left\{\eta \in I_{\gamma}: x_{\gamma+1, \eta}(\gamma)=i\right\}$, onde $i \in 2$. Note que para cada $\eta \in I_{\gamma}$, $x_{\gamma+1, \eta}(\gamma)=h\left(x_{\gamma, \eta}\right)$, logo $\left|\left\{\eta \in I_{\gamma}: x_{\gamma+1, \eta}(\gamma)=i\right\}\right|=\left|\left\{\eta \in I_{\gamma}: h\left(x_{\gamma, \eta}\right)=i\right\}\right|=\omega$ pois $h$ satisfaz c). Concluímos que o grupo $G_{\gamma+1}$ verifica as condições 1)-5). Isto completa a construção indutiva.

Seja $G:=\bigcup_{\alpha<c} G_{\alpha}$. Pela construção, $G=\left\{x_{c, \xi}: \xi<\eta\right\}$ onde para cada $\eta<\mathfrak{c}$ temos $x_{c, \eta}=\bigcup_{\alpha<c} x_{\alpha, \eta}$. Resta mostrarmos que $G$ é um grupo de van Douwen.

Como $\left\{I_{\xi}: \xi<\mathfrak{c}\right\}=[\mathfrak{c}]^{\omega}$, para cada seqüência injetora $s$ em $G$, existe $\xi<\mathfrak{c}$ de maneira tal que $s=\left\{x_{c, \eta}: \eta \in I_{\xi}\right\}$. A condição 4) implica que $x_{\mathrm{c}, \lambda_{\xi}}$ é um ponto de acumulação dessa seqüência e, em conseqüência, $G$ é enumeravelmente compacto. Agora, suponhamos por absurdo que

$$
\lim _{\eta \in I_{\xi}} x_{\llcorner, \eta}=x_{c, \lambda}
$$

Seja $F \subset \mathfrak{c}$ finito, digamos $F=\left\{\alpha_{1}, \ldots, \alpha_{n}\right\}$. Se $x_{\mathrm{c}, \lambda_{\xi}}\left(\alpha_{k}\right)=i_{k}$, temos que $\left\{\eta \in I_{\xi}: x_{c, \eta}\left(\alpha_{k}\right) \neq\right.$ $\left.i_{k}, k=1, \ldots, n\right\}$ é finito. Dado que $x_{\alpha_{k+1}, \eta} \subset x_{c, \eta}$ para todo $k=1, \ldots, n$ e todo $\eta \in I_{\xi},\left\{\eta \in I_{\xi}\right.$ : $x_{\alpha_{k+1}, \eta}\left(\alpha_{k}\right) \neq i_{k}$ \} é finito contradizendo a condição 5). Em conseqüência, qualquer seqüência não trivial em $G$ diverge.

\subsection{Grupos abelianos livres e compacidade enumerável}

Fuchs [33] mostrou que os grupos abelianos livres não triviais não podem ter uma topologia de grupo compacta. Tkačenko mostrou em 1990 [62] que sob $\mathrm{CH}$ o grupo abeliano livre cujo conjunto gerador tem cardinalidade $\mathfrak{c}$ pode ser munido de uma topologia de grupo enumeravelmente compacta, o que motivou a seguinte questão:

Questão 2.2. Em ZFC, existe uma topologia de grupo enumeravelmente compacta sobre o grupo abeliano livre cuja base tem cardinalidade $\mathfrak{c}$ ? 
Nesta seção são apresentadas duas construções que dão uma resposta afirmativa consistente com ZFC, à questão anterior. A primeira delas, deve-se a A.H. Tomita [66], quem obteve uma tal topologia assumindo $\mathrm{MA}_{\sigma-\mathrm{centrada}}$. A segunda, proposta por Koszmider et al.[46] foi obtida assumindo $\mathrm{MA}_{\text {enumerável }}$.

\section{Alguns fatos sobre grupos topológicos livres}

Os grupos topológicos livres foram introduzidos por A.A. Markov em 1941, com o intuito de estender, no contexto de grupos topológicos, a bem conhecida construção de um grupo livre da teoria de grupos. É fácil dar uma definição categórica dos grupos topológicos livres como um tipo de objeto projetivo na categoria dos grupos topológicos e homomorfismos contínuos, porém, a prova de existência de tais objetos não é trivial, sendo a primeira dificuldade no estudo destes grupos.

Definição 2.2.1. Seja $X$ um subespaço de um grupo topológico $G$. Dizemos que $G$ é um grupo topológico livre sobre $X$ se o seguinte é satisfeito:

i. $X$ gera algebricamente um subgrupo denso de $G$;

ii. para cada função contínua $f: X \rightarrow H$ onde $H$ é um grupo topológico arbitrário, existe uma extensão de $f$ a um homomorfismo contínuo $\tilde{f}: G \rightarrow H$.

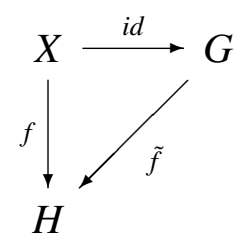

Pelo seguinte teorema, temos que um grupo topológico livre sobre um espaço $X$ é, num certo sentido, único:

Teorema 2.2.2. Sejam $G_{1}$ e $G_{2}$ grupos topológicos livres sobre um espaço de Tychonoff X. Então existe um isomorfismo topológico $\varphi: G_{1} \rightarrow G_{2}$ tal que $\varphi(x)=x$ para cada $x \in X$. Além disso, $X$ constitui um conjunto de geradores para os grupos $G_{1} e G_{2}$.

Demonstração. Pela definição 2.2.1, existem homomorfismos contínuos $\varphi_{1}: G_{1} \rightarrow G_{2}$ e $\varphi_{2}: G_{2} \rightarrow G_{1}$ cada um dos quais estende a aplicação identidade $i: X \rightarrow X$. Sejam $\psi_{1}:=$ $\varphi_{1} \circ \varphi_{2}$ e $\psi_{2}:=\varphi_{2} \circ \varphi_{1}$. Então, $\psi_{1}: G_{1} \rightarrow G_{1}$ é um homomorfismo contínuo de maneira tal que $\psi_{1} \uparrow_{X}=i d_{X}$. Dado que $X$ gera um subgrupo denso de $G_{1}$ e $G_{1}$ é de Hausdorff, $\psi_{1}$ 
constitui o automorfismo identidade de $G_{1}$. Por um argumento análogo, deduz-se que $\psi_{2}$ é o automorfismo identidade de $G_{2}, \operatorname{logo} \varphi_{1}$ e $\varphi_{2}$ são isomorfismos topológicos que mantêm fixos os pontos de $X$. Finalmente, é claro que o subgrupo denso $\langle X\rangle$ de $G_{1}$ satisfaz todas as condições da definição 2.2.1 para ser o grupo topológico livre sobre $X$. Assim, pela primeira parte do teorema temos que $G_{1}=\langle X\rangle$.

A unicidade do grupo topológico livre sobre um espaço de Tychonoff $X$ nos permite representa-lo mediante o símbolo $\mathrm{F}(X)$. Se assumimos que todos os grupos mencionados na definição 2.2.1 são Abelianos, obtemos uma definição do grupo Abeliano livre sobre $X$, que denotaremos por $\mathrm{A}(X)$.

Pelo teorema 2.2.2, temos que $X$ constitui um conjunto de geradores do grupo $\mathrm{F}(X)$; daí, cada elemento $g \in \mathrm{F}(X)$ pode ser representado na forma

$$
g=x_{1}^{\epsilon_{1}} \cdots x_{n}^{\epsilon_{n}}
$$

onde $x_{1}, \ldots, x_{n} \in X$ e $\epsilon_{1}, \ldots, \epsilon_{n} \in\{-1,1\}$. A expressão $x_{1}^{\epsilon_{1}} \cdots x_{n}^{\epsilon_{n}}$ chama-se palavra. Uma palavra $x_{1}^{\epsilon_{1}} \cdots x_{n}^{\epsilon_{n}}$ é dita reduzida se não contém um par consecutivo de símbolos da forma $x * x^{-1}$ ou $x^{-1} * x$. Daí, se uma palavra é reduzida e distinta de $\varnothing$, então é diferente da identidade do grupo $\mathrm{F}(X)$. Usando a linguagem da teoria dos grupos, dizemos que $X$ é um conjunto livre de geradores para $\mathrm{F}(X)$. Equivalentemente, desde o ponto de vista algébrico, $\mathrm{F}(X)$ é o grupo livre sobre o conjunto $X$.

No caso Abeliano, o significado da palavra "livre" é um pouco diferente. De novo, podese mostrar que se $x_{1}, \ldots, x_{n}$ são elementos distintos de $X$ e $k_{1}, \ldots, k_{n}$ são inteiros arbitrários, então a igualdade

$$
k_{1} x_{1}+k_{2} x_{2}+\cdots+k_{n} x_{n}=0_{\mathrm{A}(X)}
$$

implica que $k_{i}=0$ para cada $i=1, \ldots, n$. Em conseqüência, o grupo $\mathrm{A}(X)$ é livre de torção e, de novo, $X$ é um conjunto livre de geradores para $\mathrm{A}(X)$.

Em virtude da definição 2.2.1, a topologia do grupo $\mathrm{F}(X)$ (quando este último grupo existe) é maximal num certo sentido. A seguir, damos uma formulação matemática exata desse fato.

Fato 2.2.3 (A.A. Markov). A topologia de $\mathrm{F}(X)$ é maximal entre todas as topologias de grupo sobre $\mathrm{F}(X)$ que induzem no conjunto $X$ a topologia original.

Um teorema similar implica a existência do grupo topológico abeliano livre sobre um espaço de Tychonoff $X$. Nos anos trinta L.S. Pontryagin mostrou que todo grupo topológico de 
Hausdorff é completamente regular, motivando a questão de se todo grupo topológico Hausdorff constitui também um espaço normal. Para resolver esse problema, A.A. Markov apelou à teoria dos grupos topológicos livres com a idéia de mergulhar cada espaço de Tychonoff $X$ como subespaço fechado de um grupo topológico apropriado $G_{X}$. De fato, Markov mostrou o seguinte:

Teorema 2.2.4. Cada espaço de Tychonoff X é fechado no grupo topológico livre $\mathrm{F}(X)$ e no grupo topológico Abeliano livre $\mathrm{A}(X)$.

Depois desse resultado, Markov deu uma elegante solução ao problema da normalidade. De fato, considere-se o plano de Tychonoff $X=\left(\omega_{1}+1\right) \times\left(\omega_{1}+1\right) \backslash\left\{\left(\omega_{1}, \omega\right)\right\} ; X$ é um espaço que é completamente regular mas não normal. De acordo com o teorema precedente, o grupo topológico livre $\mathrm{F}(X)$ contém a $X$ como subconjunto fechado de modo que não pode ser um espaço normal pois, em caso contrário, $X$ seria normal.

As construções que iremos apresentar neste capítulo, utilizam homomorfismos cujo contradomínio é o grupo do circulo $\mathbb{T}$, fazendo realizações dos grupos topológicos abelianos livres como subgrupos de potências de $\mathbb{T}$. A modo de motivação, apresenta-se a seguir um fato simples que talvez ilustre num primeiro momento, uma das importâncias do grupo $\mathbb{T}$.

Lema 2.2.5. O grupo do círculo $\mathbb{T}$ contém um subgrupo algebricamente isomorfo ao grupo Abeliano livre com c geradores.

Demonstração. Consideremos o grupo aditivo $(\mathbb{R},+)$ como $\mathbb{Q}$-espaço vetorial e fixemos uma base de Hamel $X=\left\{x_{\alpha}: \alpha<\mathfrak{c}\right\}$ para $\mathbb{R}$, de maneira que $x_{0}=1$. Seja $\pi: \mathbb{R} \rightarrow \mathbb{T}$ o homomorfismo definido por $\pi(x)=e^{2 \pi i x}$ para cada $x \in \mathbb{R}$. Naturalmente $\operatorname{ker} \pi=\mathbb{Z}$. Para cada $\alpha<\mathfrak{c}$, seja $y_{\alpha}=\pi\left(x_{\alpha}\right)$. Afirma-se que o conjunto $Y=\left\{y_{\alpha}: \alpha<\mathfrak{c}\right\}$ é linearmente independente em $\mathbb{T}$, isto é, $Y$ gera o subgrupo de $\mathbb{T}$ algebricamente isomorfo ao grupo Abeliano livre de $\mathfrak{c}$ geradores. Com efeito, se $0<\alpha_{1}<\cdots<\alpha_{n}<\mathfrak{c}$ e $k_{1}, \ldots, k_{n} \in \mathbb{Z} \backslash\{0\}$, então $k_{1} x_{\alpha_{1}}+\cdots+k_{n} x_{\alpha_{n}} \notin$ $\mathbb{Z}$ e assim

$$
y_{\alpha_{1}}^{k_{1}} * \cdots * y_{\alpha_{n}}^{k_{n}}=\pi\left(k_{1} x_{\alpha_{1}}+\cdots+k_{n} x_{\alpha_{n}}\right) \neq 1
$$

Portanto, o subgrupo $\langle Y\rangle$ de $\mathbb{T}$ é isomorfo ao grupo abeliano livre abstrato de posto $\mathfrak{c}$, denotado por $A_{a}(\mathfrak{c})$.

O argumento da prova anterior também mostra que $\mathbb{T} \cong(\mathbb{Q} / \mathbb{Z}) \oplus A_{a}(\mathfrak{c})$. 


\subsubsection{Uma construção a partir de $M A_{\sigma-c e n t r a d a}$}

Neste parágrafo, consideramos uma construção feita por A.H. Tomita [66] de um subgrupo $G \subseteq \mathbb{T}^{c}$ de cardinalidade c que é livre e enumeravelmente compacto considerando a topologia de subespaço, mediante o uso de $\mathrm{MA}_{\sigma \text {-centrada }}$.

Tkačenko [62] em 1990, construiu sob CH uma topologia de grupo sobre o grupo abeliano livre com $\mathfrak{c}$ geradores. A idéia da construção foi achar um subgrupo denso e enumeravelmente compacto $G$ de $\mathbb{T}^{\mathfrak{c}}$ algebricamente isomorfo ao grupo abeliano livre de posto c; adicionalmente, esse grupo $G$ é conexo, localmente conexo, hereditariamente separável e não possui sequências convergentes além das triviais, propriedades todas do grupo de Hajnal e Juhász [39]. De fato, a construção de Tkačenko, constitui uma modificação dessa última. A escolha de $\mathbb{T}$ ao invés de $2=\{0,1\}$ na construção do grupo $G$ é forçado pelo fato de que qualquer topologia enumeravelmente compacta definida sobre um grupo abeliano livre deve ser de dimensão infinita como notou Tkačenko no trabalho citado acima.

Seguindo esse circulo de idéias, Tomita modificou o exemplo de van Douwen construído sob MA, para obter uma topologia de grupo enumeravelmente compacta e sem sequências não triviais convergentes sobre o grupo abeliano livre com c geradores, porém assumindo $\mathrm{MA}_{\sigma \text {-centrada. }}$ Vale a pena comentar a diferença entre o método que usou van Douwen e esta construção. Em cada etapa indutiva, van Douwen considera fragmentos do grupo final, isto é, faz aproximações do grupo final ao longo da indução enquanto Tomita constrói "fragmentos" de um conjunto de elementos de $\mathbb{T}^{i}$ que irão se tornar um conjunto livre de geradores para o grupo, isto é, a idéia é construir um subconjunto $X=\left\{x_{\alpha}: \alpha<\mathfrak{c}\right\} \subseteq \mathbb{T}^{\mathfrak{c}}$ de modo que o grupo $G=\langle X\rangle$ seja de van Douwen.

Daí, cada elemento desse grupo pode ser escrito como uma soma finita de elementos de $X$ e sendo cada elemento de $X$ identificado por um único ordinal em c, é possível associar para cada $x \in G$ uma função $f$ cujo domínio é um subconjunto finito de c e cuja imagem constitui um subconjunto de $\mathbb{Z} \backslash\{0\}$ de forma que

$$
x=\sum_{\xi \in \operatorname{dom}(f)} f(\xi) x_{\xi}
$$

Chamaremos a tal função a codificação de $x$; naturalmente, associamos ao elemento zero em $\mathbb{T}^{c}$, a função $\varnothing$.

De acordo com o anterior, a coleção $\mathcal{F}=\mathrm{Fn}\left([\mathrm{c}]^{<\omega}, \mathbb{Z} \backslash\{0\}\right) \backslash\{\varnothing\}$ terá um papel muito importante na construção; para simplificar um pouco a notação, representaremos por $x_{f}$ o elemento 
de $\mathbb{T}^{c}$ associado à função $f \in \mathcal{F}$.

Como foi comentado, por indução em c serão construídas aproximações dos elementos de $X$; mais explicitamente, na etapa $\alpha<\mathfrak{c}$ da construção teremos definido $\left\{x_{\beta} \uparrow_{\alpha}: \beta<\alpha\right\}$, conhecendo em cada etapa intermediária só um fragmento de $X$. Se $f \in \mathcal{F} \operatorname{e~} \operatorname{dom}(f) \subseteq \gamma$, $x_{f} \uparrow_{\gamma}:=\sum_{\mu \in \operatorname{dom}(f)} f(\mu) x_{\mu} \uparrow_{\gamma}$ vai representar o fragmento em $\mathbb{T}^{\gamma}$ do elemento em $G$ codificado por $f$. Antes de dar início à construção, convém fixar algumas notações. Seja $\left\{f_{\alpha}: \alpha<\mathfrak{c}\right\}$ uma enumeração de $\mathcal{F}$ de modo que para cada $\alpha<\mathfrak{c}, \operatorname{dom}\left(f_{\alpha}\right) \subseteq \alpha+1$. Essa enumeração vai ser usada para "codificar" qualquer elemento não nulo do grupo $G$ como combinação linear de elementos do conjunto de geradores a ser construído. Como conseqüência da anterior convenção, cada subconjunto infinito de $G$ que não contém o elemento $0 \in G$ é codificado por um subconjunto infinito de $\mathcal{F}$, pelo que vamos tomar também uma enumeração $\left\{\mathcal{F}_{\alpha}: \alpha<\mathfrak{c}\right\}$ de $[\mathcal{F}]^{\omega}$ de modo que para cada $f \in \mathcal{F}_{\alpha}, \operatorname{dom}(f) \subseteq \alpha+1$, isto é, $\bigcup_{f \in \mathcal{F}_{\alpha}} \operatorname{dom} f \subseteq \alpha+1$.

Como pretendemos que $G=\langle X\rangle$ seja um grupo abeliano livre, é preciso assegurar que todas as combinações lineares não triviais de elementos de $X$ são não nulas; daí, temos que garantir então que para cada função $f \in \mathcal{F}$ distinta de $\varnothing$, a combinação $\sum_{\mu \in \operatorname{dom}(f)} f(\mu) x_{\mu} \neq 0 \in \mathbb{T}^{c}$, condição que teremos satisfeita se existe $\beta<\mathfrak{c}$ tal que $\sum_{\mu \in \operatorname{dom}(f)} f(\mu) x_{\mu}(\beta) \neq 0 \in \mathbb{T}$. Como $|\mathcal{F}|=\mathfrak{c}$, em cada etapa da construção podemos tomar conta de uma das funções deste conjunto e garantir que o elemento do grupo codificado por ela é não nulo. Para que $X$ seja um conjunto livre de geradores resulta suficiente então que para cada $\alpha<\mathfrak{c}, x_{f_{\alpha}}(\alpha) \neq 0$. Note que na etapa $\alpha+1$, já teremos definido $x_{\mu} \uparrow_{\alpha+1}$ para cada $\mu \in \operatorname{dom}\left(f_{\alpha}\right) \subseteq \alpha$.

Finalmente, para que $G$ seja enumeravelmente compacto, devemos garantir que todo subconjunto infinito de $G$ possua um ponto de acumulação;notemos que existem c codificações possíveis para as seqüências não triviais em $G$, mas, por razões técnicas só podemos tomar conta de uma quantidade menor que $\mathfrak{c}$ seqüências em cada etapa. De fato, na etapa $\alpha+1$, "prometemos” que $x_{\alpha}$ será ponto de acumulação da seqüência $\left\{x_{f}: f \in \mathcal{F}_{\alpha}\right\}$. Em cada etapa $\gamma<\mathfrak{c}$, tomaremos conta só das seqüências codificadas por $\mathcal{F}_{\alpha}$ para cada $\alpha<\gamma$.

A seguinte observação simples, resulta ser muito útil para todas as construções subseqüentes:

Lema 2.2.6. Seja $y \in \mathbb{T}^{\beta} e\left\{y_{n}: n \in \omega\right\} \subseteq \mathbb{T}^{\beta}$, onde $\beta<\mathfrak{c}$ é um ordinal limite. Então y é um ponto de acumulação da seqüência $\left(y_{n}\right)_{n \in \omega}$ se e somente se $y \uparrow_{\alpha}$ é ponto de acumulação de $\left\{y_{n} \uparrow_{\alpha}: n \in \omega\right\}$ para cada $\alpha<\beta$.

Pelo resultado anterior, é suficiente escolher $x_{\alpha}$ como ponto de acumulação da seqüência em $\mathbb{T}^{\alpha}$ codificada por $\mathcal{F}_{\alpha}$ e mostrar por indução que se $x_{\alpha} \uparrow_{\gamma}$ é ponto de acumulação para a 
seqüência de $\mathbb{T}^{\gamma}$ codificada por $\mathcal{F}_{\alpha}$ é possível definir as $\gamma$-ésimas coordenadas dos pontos em questão de forma tal que $x_{\alpha} \uparrow_{\gamma+1}$ seja ponto de acumulação da seqüência em $\mathbb{T}^{\gamma+1}$ codificada por $\mathcal{F}_{\alpha}$. Vamos reinterpretar esta última condição em termos de subconjuntos de $\mathcal{F}_{\alpha}$. No que segue, $\|t\|$ representa a norma euclidiana de um ponto $t \in \mathbb{R}^{2}$.

Para cada $\alpha \leq \gamma, F \in[\gamma]^{<\omega}$ e $k \in \omega$, definimos

$$
E(\alpha, F, k)=\left\{f \in \mathcal{F}_{\alpha}: \text { para todo } \mu \in F,\left\|x_{f}(\mu)-x_{\alpha}(\mu)\right\|<\frac{1}{k+1}\right\}
$$

Lema 2.2.7. Seja $\alpha \leq \gamma$. Então, $x_{\alpha} \uparrow_{\gamma}$ é ponto de acumulação da seqüência em $\mathbb{T}{ }^{\gamma}$ codificada por $\mathcal{F}_{\alpha}$ se e somente se para cada $F \in[\gamma]^{<\omega}$ e $k \in \omega,|E(\alpha, F, k)|=\omega$.

Demonstração. Seja $F \in[\gamma]^{<\omega}, k \in \omega$. Considere a vizinhança aberta básica de $x_{\alpha} \uparrow_{\gamma}$ em $\mathbb{T}^{\gamma}$ dada por

$$
U_{\gamma}\left(x_{\alpha}, F, k\right)=\left\{g \in \mathbb{T}^{\gamma}: \forall \theta \in F,\left\|g(\theta)-x_{\alpha} \uparrow_{\gamma}(\theta)\right\|<\frac{1}{k+1}\right\}
$$

Notemos que $x_{\alpha} \uparrow_{\gamma}$ é ponto de acumulação da seqüência em $\mathbb{T}^{\gamma}$ codificada por $\mathcal{F}_{\alpha}$ se e somente se $U_{\gamma}\left(x_{\alpha}, F, k\right) \cap\left\{x_{f} \uparrow_{\gamma}: f \in \mathcal{F}_{\alpha}\right\}$ é infinito; posto que cada $f \in \mathcal{F}_{\alpha}$ representa um único objeto de $\mathbb{T}^{\gamma}$ temos que $\left\{f \in \mathcal{F}_{\alpha}: x_{f} \uparrow_{\gamma} \in U\left(x_{\alpha} \uparrow_{\gamma}, F, k\right)\right\}=E(\alpha, F, k)$ é infinito.

De acordo com as considerações anteriores, vamos provar o seguinte resultado:

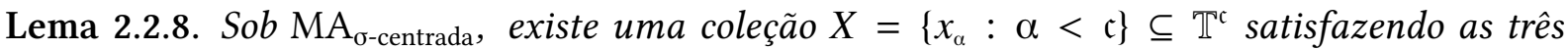
condições seguintes:

A. para cada $\alpha<\mathfrak{c}, x_{f \alpha}(\alpha) \neq 0$;

B. para cada $\alpha<\gamma<\mathfrak{c}, F \in[\gamma]^{<\omega}$ e $k \in \omega$ o conjunto $E(\alpha, F, k)$ é infinito;

C. Se $\alpha<\gamma$ e a condição B) é satisfeita para $\alpha, F \in[\gamma]^{<\omega}$ e $k \in \omega$ então o conjunto $\left\{f \in E(\alpha, F, f):\left\|x_{f}(\gamma)-x_{\alpha}(\gamma)\right\|<\frac{1}{k+1}\right\}$ é infinito.

Demonstração. A prova é por indução em c. Ao longo desta prova, é necessário mostrar muitos outros resultados que incluímos aqui para maior compreensão do leitor.

Na etapa 0 , não temos nada a fazer. Seja $\gamma<\mathfrak{c}$ e suponhamos que para cada $\beta<\gamma$ os elementos $\left\{x_{\alpha} \uparrow_{\beta}: \alpha<\beta\right\}$ satisfazem as condições $\left.\left.A\right)-C\right)$.

Observe que na etapa $\gamma$, para cada $\alpha<\gamma$, temos que definir $x_{\alpha} \uparrow_{\gamma}$.

Se $\gamma$ é limite, defina $x_{\alpha} \uparrow_{\gamma}:=\bigcup_{\alpha<\beta<\gamma} x_{\alpha} \uparrow_{\beta}$. Naturalmente, $\left\{x_{\alpha} \uparrow_{\gamma}: \alpha<\gamma\right\}$ satisfaz A) - C). 
Suponhamos agora que $\gamma=\beta+1$. Por hipótese, temos definido a coleção $\left\{x_{\alpha} \uparrow_{\beta}: \alpha<\beta\right\}$ satisfazendo A) - C). Daí, a seqüência em $\mathbb{T}^{\beta}$ codificada por $\mathcal{F}_{\beta}$ já está definida e podemos fixar um ponto de acumulação $y \in \mathbb{T}^{\beta}$ dela. Seja $x_{\beta} \uparrow_{\beta}:=y$.

Notemos que B) é satisfeita para o ordinal $\beta$; de fato, se $F \in[\gamma]^{<\omega}$ e $k \in \omega$, temos

$$
\begin{aligned}
E(\beta, F, k) & =\left\{f \in \mathcal{F}_{\beta}: \forall \xi \in F\left\|\sum_{\mu \in \operatorname{dom} f} f(\mu) x_{\mu}(\xi)-x_{\beta}(\xi)\right\|<\frac{1}{k+1}\right\} \\
& =\left\{f \in \mathcal{F}_{\beta}: \forall \xi \in F\left\|\sum_{\mu \in \operatorname{dom} f} f(\mu) x_{\mu}(\xi)-y(\xi)\right\|<\frac{1}{k+1}\right\} \\
& =\left\{f \in \mathcal{F}_{\beta}: \sum_{\mu \in \operatorname{dom} f} f(\mu) x_{\mu} \in U(y, F, k)\right\}
\end{aligned}
$$

é um conjunto infinito.

Fixemos uma base enumerável $\mathcal{B}$ de $\mathbb{T}$, assumindo, por conveniência, que $\mathbb{T} \in \mathcal{B}$. Para mostrarmos o resultado, é preciso definir uma ordem parcial $\mathbb{P}$ e conjuntos densos que serão usados para achar uma função $\phi$ que vai permitir estender os elementos que já temos construído em $\mathbb{T} \gamma$. Fixemos uma base enumerável $\mathcal{B}$ para $\mathbb{T}$ e suponhamos que $\mathbb{T} \in \mathcal{B}$.

Seja

$$
\mathbb{P}_{\gamma}=\operatorname{Fn}(\gamma, \mathcal{B})
$$

Dados $p, q \in \mathbb{P}_{\gamma}$, definimos a relação de ordem $\leq$ por $p \leq q$ se e somente $\operatorname{se} \operatorname{dom}(q) \subseteq \operatorname{dom}(p)$ e para cada $\mu \in \operatorname{dom}(q), p(\mu)=q(\mu)$ ou $\overline{p(\mu)} \subseteq q(\mu)$.

Notemos que em cada etapa indutiva, é definida uma ordem parcial distinta. Em consideração dessa observação, para simplificar a notação tomemos $\mathbb{P}:=\mathbb{P}_{\gamma}$.

Lema 2.2.9. A ordem parcial $\mathbb{P}_{\gamma}$ é $\sigma$-centrada.

Demonstração. Consideremos o espaço $\mathcal{B}^{\gamma}$, onde $\mathcal{B}$ é munido da topologia discreta; notemos que este espaço constitui precisamente o conjunto de todas as funções cujo domínio é $\gamma$ e cuja imagem é um subconjunto de $\mathcal{B}$. A base canônica de $\mathcal{B}^{\gamma}$ está constituída por conjuntos da forma

$$
V_{q}=\left\{f \in \mathcal{B}^{\gamma}: q \subseteq f\right\}
$$

onde $\operatorname{dom} q \in[\gamma]^{<\omega}$, e im $q \subseteq \mathcal{B}$, isto é, $q \in \mathbb{P}$.

Como $\mathcal{B}$ é enumerável e $\gamma<\mathfrak{c}$, temos que $\mathcal{B}^{\gamma}$ constitui um espaço separável, logo existe uma coleção enumerável $S=\left\{s_{n}: n \in \omega\right\} \subseteq \mathcal{B}^{\gamma}$ densa nesse espaço. Para cada $n \in \omega$, seja

$$
\mathbb{P}_{n}=\left\{p \in \mathbb{P}: p \subseteq s_{n}\right\}
$$


Note-se que $\mathbb{P}=\bigcup_{n \in \omega} \mathbb{P}_{n}$; de fato, para cada $q \in \mathbb{P}$, existe $m \in \omega$ tal que $s_{m} \in V_{q}$, isto é, $q \subseteq s_{m}$, $\operatorname{logo} q \in \mathbb{P}_{n}$. Vejamos agora que para cada $n \in \omega, \mathbb{P}_{n}$ é centrado. Se $B \in\left[\mathbb{P}_{n}\right]^{<\omega}$, tomemos $F=\bigcup_{p \in B} \operatorname{dom} p \subseteq \gamma$; seja $q:=s_{n} \uparrow_{F} \in \mathcal{B}^{\gamma}$. Notemos que $F=\operatorname{dom} q$ é finito e além disso $q \subseteq s_{n}$, isto é, $q \in \mathbb{P}_{n}$; para cada $p \in B, p \subseteq s_{n}$, logo sempre que $\alpha \in \operatorname{dom} p, p(\alpha)=s_{n}(\alpha)=q(\alpha)$; posto que dom $p \subseteq F$ temos $q \leq p$ para todo $p \in B$, isto é, $q$ é minorante de $B$ em $\mathbb{P}_{n}$.

O seguintes dois lemas, mostram quais são os conjuntos densos em $\mathbb{P}$ que fazem possível construir $\phi$; obviamente, nas hipóteses deles estamos assumindo sempre que $\gamma=\beta+1<\mathfrak{c}$.

Lema 2.2.10. Seja $\mathbb{P}$ como acima. Então

1. para cada $\zeta<\gamma$, o conjunto $D_{\zeta}=\{p \in \mathbb{P}: \zeta \in \operatorname{dom}(p)\}$ é denso em $\mathbb{P}$;

2. o conjunto $\mathcal{O}=\left\{p \in \mathbb{P}: 0 \notin \sum_{\mu \in \operatorname{dom} f_{\beta}} f_{\beta}(\mu) p(\mu)\right\}$ é denso e aberto em $\mathbb{P}$.

Demonstração. Tomemos um elemento $q \in \mathbb{P}$.

1. Se $\zeta \notin \operatorname{dom} q$, definimos $p:=q \cup\{(\zeta, \mathbb{T})\}$. Naturalmente, $p \in D_{\zeta}$ e $p \leq q$.

2. Primeiro, vamos construir $\tilde{q} \leq q$ satisfazendo $\operatorname{dom} f_{\beta} \subseteq \operatorname{dom} \tilde{q}$; seja $\mu_{1}$ o menor ordinal tal que $\mu_{1} \in \operatorname{dom} f_{\beta} \backslash \operatorname{dom} q$. Por 1$), D_{\mu_{1}}$ é denso em $\mathbb{P}$, logo existe $q_{1} \leq q$ tal que $\mu_{1} \in$ $\operatorname{dom} q_{1}$; dado que $\operatorname{dom} f_{\beta}$ é finito, após aplicar o processo anterior um número finito de vezes, obtemos finalmente uma extensão $\tilde{q} \in \mathbb{P} \operatorname{de} q$ satisfazendo $\operatorname{dom} f_{\beta} \subseteq \operatorname{dom} \tilde{q}$.

Suponhamos que $0 \in \sum_{\mu \in \operatorname{dom} f_{\beta}} f_{\beta}(\mu) \tilde{q}(\mu)$ e dom $f_{\beta}=\left\{\mu_{1}, \ldots, \mu_{n}\right\}$. Dado que $U=\sum_{i=1}^{n} f_{\beta}\left(\mu_{i}\right) \tilde{q}\left(\mu_{i}\right) \subseteq$ $\mathbb{T}$ é aberto, podemos tomar $z \in U \backslash\{0\}$ e uma vizinhança aberta $V$ de $z$ satisfazendo $\bar{V} \subseteq U \neq\{0\}$. Como a aplicação $g: \mathbb{T}^{n} \rightarrow \mathbb{T}$ determinada pelos coeficientes $\left\{f\left(\mu_{i}\right)\right.$ : $\left.\mu_{i} \in \operatorname{dom} f_{\beta}\right\} \subseteq \mathbb{Z} \backslash\{0\}$ é contínua, pela regularidade de $\mathbb{T}$ obtemos abertos $V_{1}, \ldots, V_{n} \in \mathcal{B}$ de modo que $\overline{V_{i}} \subseteq \tilde{q}\left(\mu_{i}\right)$ para cada $i=1, \ldots, n$ e $z \in \sum_{i=1}^{n} f_{\beta}\left(\mu_{i}\right) V_{i} \subseteq V$. Para cada $\mu \in$ $\operatorname{dom} \tilde{q} \backslash \operatorname{dom} f_{\beta}$, seja $p(\mu)=\tilde{q}(\mu)$ e, para cada $i=1, \ldots, n$, definimos $p\left(\mu_{i}\right)=V_{i}$. É claro que $p \leq \tilde{q}$ e, como $\sum_{i=1}^{n} f_{\beta}\left(\mu_{i}\right) p\left(\mu_{i}\right) \subseteq V \subseteq U \backslash\{0\}, p \in \mathcal{O}$. Se $p \in \mathcal{O}$, e $r \leq p$, naturalmente $\sum_{\mu \in \operatorname{dom} f_{\beta}} f_{\beta}(\mu) r(\mu) \subseteq \sum_{\mu \in \operatorname{dom} f_{\beta}} f_{\beta}(\mu) p(\mu)$, de onde $0 \notin \sum_{\mu \in \operatorname{dom} f_{\beta}} f_{\beta}(\mu) r(\mu)$, isto é, $r \in \mathcal{O}$; portanto, $\mathcal{O}$ é aberto em $\mathbb{P}$.

Por hipótese de indução, para cada $\alpha<\gamma, F \in[\beta]^{<\omega}$ e $k \in \omega$, o conjunto $E(\alpha, F, k)$ é infinito, logo podemos tomar uma partição dele em subconjuntos infinitos $\{E(\alpha, F, k, n): n \in$ $\omega\}$.

Seja $\mathscr{C}=\left\{E(\alpha, F, k, n): \alpha<\gamma, F \in[\beta]^{<\omega}, k, n \in \omega\right\}$. Note que $|\mathscr{C}| \leq \gamma$. Considerando esta coleção, obtemos convenientemente mais subconjuntos densos em $\mathbb{P}$. 
Lema 2.2.11. Para cada $E \in \mathscr{C}$ e $U \in \mathcal{B}$, o conjunto

$$
\mathbb{D}_{E, U}=\left\{p \in \mathbb{P}: \text { existe } f \in E \text { tal que } \sum_{\mu \in \operatorname{dom} f} f(\mu) p(\mu) \subseteq U\right\}
$$

é denso em $\mathbb{P}$.

Demonstração. O argumento a seguir é similar ao de Tkačenko em [62]. Fixemos $E \in \mathscr{C}$, $U \in \mathcal{B}$ e $q \in \mathbb{P}$. Temos que considerar dois casos:

- Caso 1: Existe um subconjunto infinito $E_{1} \subseteq E$ de modo que $\operatorname{dom} f \neq \operatorname{dom} g$ sempre que $f \neq g \in E_{1}$. Tome $f \in E_{1}$ e $\mu<\gamma$ tal que $\mu \in \operatorname{dom} f \backslash \operatorname{dom} q$; seja $D:=\operatorname{dom} q \cup \operatorname{dom} f$. Para cada $\xi \in \operatorname{dom} q$, escolha $a_{\xi} \in q(\xi)$ e para cada $\xi \in D \backslash(\operatorname{dom} q \cup\{\mu\})$, tome $a_{\xi}=0$. Escolha $a_{\mu} \in \mathbb{T}$ de modo que $\sum_{\xi \in \operatorname{dom} f} f(\xi) a_{\xi} \in U$. Pela continuidade da soma e lembrando que $\mathbb{T}$ é regular, para cada $\xi \in D$ existe $U_{\xi} \in \mathcal{B}$ de modo que $a_{\xi} \in U_{\xi}$ e $\left(U_{\xi}\right) \subseteq q(\xi)$ satisfazem

$$
\sum_{\xi \in \operatorname{dom} f} f(\xi) U_{\xi} \subseteq U
$$

Defina $p=\left\{\left(\xi, U_{\xi}\right): \xi \in D\right\}$. Naturalmente, $p \leq q$ e $p \in \mathbb{D}_{E, U}$.

- Caso 2: Existem um subconjunto infinito $E_{2} \subseteq E$ e $D \in[\mathfrak{c}]^{<\omega}$ tal que $D=\operatorname{dom} g$ para toda $g \in E_{2}$. Se $D \nsubseteq \operatorname{dom} q$, podemos estender $q$ tomando $\tilde{q}(\mu)=\mathbb{T}$ nos pontos $\mu \in$ $D \backslash \operatorname{dom} q$, logo podemos assumir sem perda de generalidade que $D \subseteq \operatorname{dom} q$. Como todas as funções no conjunto infinito $E_{2}$ são distintas e tem por domínio finito comum a $D$, existe $\mu \in D$ de modo que o conjunto $\left\{f(\mu): f \in E_{2}\right\} \subset \mathbb{Z}$ não é limitado. Dai, podemos tomar $g \in E_{2}$ tal que $g(\mu) q(\mu)=\mathbb{T}$.

Para cada $\xi \in D \backslash\{\mu\}$, fixe $a_{\xi} \in q(\xi)$. Pela continuidade da soma, podemos escolher $a_{\mu} \in q(\mu)$ tal que $\sum_{\xi \in D \backslash\{\mu\}} g(\xi) a_{\xi}+g(\mu) a_{\mu} \in U$. De novo, pela continuidade da soma, para cada $\xi \in D \backslash\{\mu\}$ podemos tomar $U_{\xi} \in \mathcal{B}$, tal que $a_{\xi} \in U_{\xi}$ e $\overline{U_{\xi}} \subseteq q(\xi)$, tomando também para $\mu$ um aberto $U_{\mu} \in \mathcal{B}$ de modo que $a_{\mu} \in U_{\mu}$ e $\overline{U_{\mu}} \subseteq q(\mu)$ são tais que $\sum_{\xi \in D} g(\xi) U_{\xi} \subseteq U$.

Defina $p=\left\{\left(\xi, U_{\xi}\right): \xi \in D\right\} \cup\{(\kappa, q(\kappa)): \kappa \in \operatorname{dom} q \backslash D\}$. Da maneira em que foi construída a função $p$, é claro que $p \leq q$ e $g \in E_{2} \subseteq E$ satisfaz

$$
\sum_{\xi \in D} g(\xi) p(\xi)=\sum_{\xi \in D} g(\xi) U_{\xi} \subseteq U
$$

isto é, $p \in \mathbb{D}_{E, U}$. 
Por último, temos o resultado que envolve $\mathrm{MA}_{\sigma-c e n t r a d a}$ neste passo da construção indutiva:

Lema 2.2.12. Assuma $\mathrm{MA}_{\sigma-\mathrm{centrada}}$. Considerando as notações anteriores, se $\gamma=\beta+1<\mathfrak{c}$ existe uma função $\phi: \gamma \rightarrow \mathbb{T}$ satisfazendo:

i. $\sum_{\mu \in \operatorname{dom}\left(f_{\beta}\right)} f_{\beta}(\mu) \phi(\mu) \neq 0$;

ii. para cada $\alpha \leq \beta, F \in[\beta]^{<\omega}$ e $k \in \omega$ o conjunto

$$
\left\{f \in E(\alpha, F, k):\left\|\sum_{\mu \in \operatorname{dom}(f)} f(\mu) \phi(\mu)-\phi(\alpha)\right\|<\frac{1}{k+1}\right\}
$$

é infinito.

Demonstração. Notemos que em virtude dos lemas 2.2.10 e 2.2.11, as coleções $\mathscr{D}=\left\{D_{\zeta}: \zeta<\right.$ $\gamma\} \cup\{\mathcal{O}\}$ e $\left\{\mathbb{D}_{E, U}: E \in \mathscr{C}, U \in \mathcal{B}\right\}$, estão constituídas por elementos densos na ordem $\mathbb{P}$; dado que a cardinalidade de cada uma delas não ultrapassa $|\gamma|<\mathfrak{c}$ e a ordem $\mathbb{P}$ é $\sigma$-centrada, ao estarmos assumindo $\mathrm{MA}_{\sigma \text {-centrada }}$, existe um filtro $\mathbb{G} \subseteq \mathbb{P}$ que interseta cada conjunto dessas coleções.

Seja $\Phi: \gamma \rightarrow \wp(\mathcal{B})$ a aplicação definida para cada $\xi<\gamma$ por

$$
\Phi(\xi)=\{p(\xi): \xi \in \operatorname{dom} p, p \in \mathbb{G}\}
$$

Se $\xi<\gamma$, afirma-se que $\bigcap \Phi(\xi) \neq \varnothing$; em primeiro lugar, notemos que $\Phi(\xi) \neq \varnothing$, pois $\mathbb{G}$ interseta a $D_{\xi}$. Como $\Phi(\xi) \subseteq \mathcal{B}$ é enumerável, podemos escrever $\Phi(\xi)=\left\{p_{i}(\xi): i \in \omega\right\}$. Como $\mathbb{G}$ é um filtro e $p_{1}, p_{2} \in \mathbb{G}$, existe $r \in \mathbb{G}$ tal que $r \leq p_{i}$ onde $i=1$, 2; daí, $r(\xi) \subseteq p_{1}(\xi) \cap p_{2}(\xi)$ (ou, mais ainda, $\overline{r(\xi)} \subseteq p_{1}(\xi) \cap p_{2}(\xi)$ ). Indutivamente, dado $k \in \omega$, podemos achar $s \in \mathbb{G}$ tal que $s(\xi) \subseteq p_{1}(\xi) \cap p_{2}(\xi) \cap \cdots \cap p_{k}(\xi)$. Como $\mathbb{T}$ é regular, para cada $k \in \omega$ é possível achar um subconjunto fechado não vazio $F_{k} \subseteq \mathbb{T}$ de modo que $F_{k} \subseteq p_{1}(\xi) \cap p_{2}(\xi) \cap \cdots \cap p_{k}(\xi)$. A compacidade de $\mathbb{T}$, e o fato de ser um espaço de Hausdorff, implica que $\bigcap \Phi(\xi) \neq \varnothing$, logo podemos definir uma função $\phi_{\mathbb{G}}: \gamma \rightarrow \mathbb{T}$ escolhendo para cada $\xi<\gamma, \phi_{\mathbb{G}}(\xi) \in \bigcap \Phi(\xi)$.

Notemos que existe $q \in \mathbb{G} \cap \mathcal{O}$; daí, se $z \in \bigcap \Phi(\beta), z \in q(\beta)$, de modo que $\sum_{\mu \in \operatorname{dom} f_{\beta}} f_{\beta}(\mu) z \neq 0$; em particular, $\sum_{\mu \in \operatorname{dom} f_{\beta}} f_{\beta}(\mu) \phi_{\mathbb{G}}(\mu) \neq 0$, isto é, $\phi_{\mathbb{G}}$ satisfaz a condição i).

Sejam $\alpha \leq \beta, F \in[\beta]^{<\omega}$ e $k \in \omega$. Consideremos a vizinhança aberta de $\phi_{\mathbb{G}}(\alpha)$ em $\mathbb{T}$, $U^{\prime}=\left\{z \in \mathbb{T}:\left\|z-\phi_{\mathbb{G}}(\alpha)\right\|<\frac{1}{k+1}\right\}$ e seja $U \in \mathcal{B}$ tal que $U \subseteq U^{\prime}$. Para cada $n \in \omega$, seja $E_{n}=E(\alpha, F, k, n)$; dado que $\mathbb{D}_{E_{n}, U}$ é denso em $\mathbb{P}$, existe $p_{n} \in \mathbb{G} \cap \mathbb{D}_{E_{n}, U}$, i.e. existe $f_{n} \in E_{n}$ tal que $\sum_{\mu \in \operatorname{dom} f_{n}} f_{n}(\mu) p_{n}(\mu) \subseteq U$. Notemos que para cada $\mu \in \operatorname{dom} f_{n}, p_{n}(\mu) \in \Phi(\mu), \operatorname{logo} \phi_{G} \in p_{n}(\mu)$ de 
modo que $\sum_{\mu \in \operatorname{dom} f_{n}} f_{n}(\mu) \phi_{G}(\mu) \in U \subseteq U^{\prime}$, isto é $\left\|\sum_{\mu \in \operatorname{dom} f_{n}} f_{n}(\mu) \phi_{\mathbb{G}}(\mu)-\phi_{\mathbb{G}}(\alpha)\right\|<\frac{1}{k+1}$. Dado que $\left\{E_{n}: n \in \omega\right\}$ constitui uma partição de $E(\alpha, F, k)$, a coleção de funções $\left\{f_{n}: n \in \omega\right\} \subseteq\{f \in$ $\left.E(\alpha, F, k):\left\|\sum_{\mu \in \operatorname{dom}(f)} f(\mu) \phi_{\mathbb{G}}(\mu)-\phi_{\mathbb{G}}(\alpha)\right\|<\frac{1}{k+1}\right\}$ é infinita, ou seja, $\phi_{\mathbb{G}}$ satisfaz a condição ii).

Tomando $\phi:=\phi_{\mathbb{G}}$ conclui-se a prova.

Voltamos à etapa $\gamma=\beta+1$ da nossa construção. Recapitulando, do passo anterior da indução, a coleção $\left\{x_{\alpha} \uparrow_{\beta}: \alpha<\beta\right\}$ já tinha sido definida satisfazendo A) - C) e definimos $x_{\beta} \uparrow_{\beta}:=$ $y$ onde $y$ é um ponto de acumulação em $\mathbb{T}^{\beta}$ da seqüência ali codificada por $\mathcal{F}_{\beta}$. Em seguida, foi provado que a condição B) vale para $\beta$.

Como assumimos $\mathrm{MA}_{\sigma-\text { centrada }}$, existe $\phi: \gamma \rightarrow \mathbb{T}$ satisfazendo as condições i) e ii) do lema 2.2.12. Para cada $\alpha<\gamma$, tomemos $x_{\alpha} \uparrow_{\gamma}:=x_{\alpha} \uparrow_{\beta}^{-} \phi(\alpha)$. É suficiente ver que as condições A) e C) são satisfeitas no caso $\alpha=\beta$ :

A. Visto que $\phi$ satisfaz a condição 1) do lema 2.2.7, $\sum_{\mu \in \operatorname{dom} f_{\beta}} f_{\beta}(\mu) \phi(\mu)=\sum_{\mu \in \operatorname{dom} f_{\beta}} f_{\beta}(\mu) x_{\mu} \Upsilon_{\gamma}(\beta)=$ $x_{f_{\beta}}(\beta) \neq 0$.

C. Sejam $F \in[\gamma]^{<\omega}, k \in \omega$. Sabemos que a condição B) é satisfeita por $\beta$; pelo item b) do lema 2.2 .7 o conjunto

$$
\begin{aligned}
\left\{f \in E(\beta, F, k):\left\|\sum_{\mu \in \operatorname{dom} f} f(\mu) \phi(\mu)-\phi(\beta)\right\|\right. & \left.<\frac{1}{k+1}\right\} \\
= & \left\{f \in E(\beta, F, k):\left\|\sum_{\mu \in \operatorname{dom} f} f(\mu) x_{\mu}(\beta)-x_{\beta}(\beta)\right\|<\frac{1}{k+1}\right\} \\
= & \left\{f \in E(\beta, F, k):\left\|x_{f}(\beta)-x_{\beta}(\beta)\right\|<\frac{1}{k+1}\right\}
\end{aligned}
$$

é infinito, logo a condição C) é satisfeita para $\beta$.

Por indução, temos construído então uma coleção $\left\{x_{\alpha}: \alpha<\mathfrak{c}\right\}$ satisfazendo as condições A) - C).

O seguinte lema, mostra uma sutileza da construção desse conjunto de geradores que vai nos permitir concluir que o grupo $G=\langle X\rangle$ não possui seqüências não triviais convergentes.

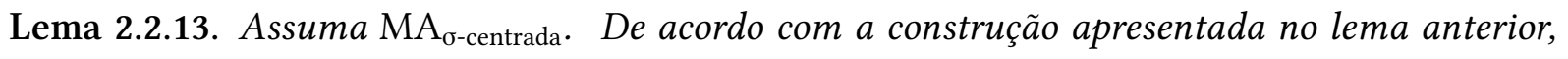
para cada $\gamma \in[\omega, \mathfrak{c})$, o conjunto $\left\{x_{f}(\gamma): f \in \mathcal{F} \gamma\right\}$ é denso em $\mathbb{T}$.

Demonstração. Sejam $\xi \in[\gamma+1, \mathfrak{c}), V \in \mathcal{B}$ e $F \in[\xi]^{<\omega}$, de modo que para cada $\alpha \in F, \gamma \leq \alpha$. Seja $E=(\gamma, F, 1)$. De acordo com a $\xi+1$-ésima etapa da construção, temos que $\mathbb{D}_{E, V}$ é denso 
em $\mathbb{P}_{\xi+1}$, logo existe $p \in \mathbb{G} \cap \mathbb{D}_{E, V}$ onde $\mathbb{G}$ representa o filtro genérico dado por $M A_{\sigma \text {-centrada }}$ nessa etapa. Em conseqüência, existe $f \in E \subseteq \mathcal{F}_{\gamma}$ tal que $\sum_{\mu \in \operatorname{dom} f} f(\mu) p(\mu) \subseteq V$.

De acordo com a construção, temos também que $\bigcup_{f \in F_{\gamma}} \operatorname{dom} f \subseteq \gamma$. Além disso, para cada $\mu \in \operatorname{dom} f, \phi_{\mathbb{G}}(\mu) \in p(\mu), \log o$

$$
\sum_{\mu \in \operatorname{dom} f} f(\mu) \phi_{\mathrm{G}}(\mu)=\sum_{\mu \in \operatorname{dom} f} f(\mu) x_{\mu}(\gamma)=x_{f}(\gamma) \in V
$$

Dado que $\mathcal{B}$ é base de $\mathbb{T}$ e $f \in \mathcal{F}_{\gamma}$, obtemos o resultado.

Teorema 2.2.14. Assumindo $\mathrm{MA}_{\sigma-c e n t r a d a}$, existe $G \subseteq \mathbb{T}^{c}$ tal que $G$ é um grupo abeliano livre e de van Douwen.

Demonstração. Pelo lema 2.2.8 temos que o grupo $G$ gerado pela coleção $X=\left\{x_{\alpha}: \alpha<\mathfrak{c}\right\}$ é um grupo abeliano livre e enumeravelmente compacto. Resta mostrar que neste grupo as únicas seqüências convergentes são as triviais.

Seja $\left\{y_{n}: n \in \omega\right\} \subseteq G$ uma seqüência injetora cujos elementos são todos não nulos. Seja $\alpha \in[\omega, \mathfrak{c})$ tal que a seqüência anterior está codificada pela coleção $\mathcal{F}_{\alpha}$, isto é, $\mathcal{F}_{\alpha}=\left\{f_{n}: n \in \omega\right\}$ onde para cada $n \in \omega, y_{n}=x_{f_{n}}$. Pelo lema 2.2.13, o conjunto $\left\{x_{f}(\alpha): f \in \mathcal{F}_{\alpha}\right\}$ é denso em $\mathbb{T}$; observemos que para cada $n \in \omega$,

$$
y_{n}(\alpha)=x_{f_{n}}(\alpha)
$$

Sendo o conjunto $\left\{y_{n}(\alpha): n \in \omega\right\}$ denso em $\mathbb{T}$, a seqüência $\left\{y_{n}: n \in \omega\right\}$ diverge.

\subsubsection{Uma construção a partir de $M A_{\text {enumerável }}$}

Neste parágrafo, exibiremos a construção proposta por P. Koszmider et. al. [46] de uma topologia de grupo definida sobre $A(\mathfrak{c})$ munido da qual resulta ser um grupo de van Douwen, assumindo a asserção $M A_{\text {enumerável }}$.

Como nos exemplos precedentes e mais alguns da literatura, este exemplo é construído por indução em $\mathfrak{c}$, definindo em cada etapa uma função conveniente para obter as novas coordenadas dos elementos que irão estar no grupo. Contudo, uma diferença substancial aparece nela. No exemplo de van Douwen, notamos que o domínio da função $h$ cresce através da indução em c. Na construção de Tomita sob $\mathrm{MA}_{\sigma-c e n t r a d a}$, observamos que na etapa $\alpha$ aparece um novo elemento $x_{\alpha} \in \mathbb{T}^{c}$ que conhecemos parcialmente e cujo domínio vai se acrescentando nas etapas seguintes, de modo similar do que na construção de van Douwen, sem fazer modificações aos pontos já definidos dele. 
Para poder trabalhar com $\mathrm{MA}_{\text {enumerável }}$ e produzir um grupo topológico enumeravelmente compacto cujo quadrado não satisfaz esta propriedade, Hart e van Mill [40] fizeram uso de um grupo $\omega$-limitado que toma conta dos pontos de acumulação de um grupo enumerável prefixado; naturalmente, o uso desse grupo $\omega$-limitado faz com que no grupo exista um grande número de seqüências não triviais convergentes. Para superar esse impasse, uma idéia surge da utilização de submodelos elementares: em cada etapa do processo indutivo se toma conta de uma quantidade enumerável de pontos, mas, ao longo da construção, esses pontos irão variar.

Como na seção precedente, se construirá uma família livre $X=\left\{x_{\alpha}: \alpha<\mathfrak{c}\right\} \subseteq \mathbb{T}^{\mathfrak{c}}$, de modo que o grupo $G:=\langle X\rangle$, considerado como subespaço de $\mathbb{T}^{c}$, seja de van Douwen.

Lembramos, da seção precedente, que cada $x \in G$ não nulo é codificado por uma função em $\operatorname{Fn}(\mathfrak{c}, \mathbb{Z} \backslash\{0\})$, de modo tal que

$$
x=\sum_{\mu \in \operatorname{dom} f} f(\mu) x_{\mu}
$$

Com isso, é possível associar a cada seqüência de elementos em $G$, digamos $\left\{z_{n}: n \in \omega\right\}$, uma função $h: \omega \rightarrow \operatorname{Fn}(\mathfrak{c}, \mathbb{Z} \backslash\{0\})$ de modo que para cada $n \in \omega, h(n)$ é a codificação de $z_{n}$. De acordo com isto, fixemos uma enumeração $\left\{h_{\xi}: \omega \leq \xi<\mathfrak{c}\right\}$ das funções injetoras que pertencem a $(\operatorname{Fn}(\mathfrak{c}, \mathbb{Z} \backslash\{0\}))^{\omega}$ de modo que para cada $\xi \in[\omega, \mathfrak{c}), \bigcup_{n \in \omega} \operatorname{dom} h_{\xi}(n) \subseteq \xi$.

Notemos que os $h_{\xi}^{\prime} s$ codificam todas as seqüências injetoras no grupo gerado por $X$ que não contém 0 . Tomemos também uma enumeração $\left\{j_{\xi}: \xi \in[\omega, \mathfrak{c})\right\}$ de $\operatorname{Fn}\left([\mathfrak{c}]^{<\omega}, \mathbb{Z} \backslash\{0\}\right)$, satisfazendo dom $j_{\xi} \subseteq \xi$. De maneira similar, é de notar que $X$ é um conjunto livre de geradores se para cada $\xi \in[\omega, \mathfrak{c}), \sum_{\mu \in \operatorname{dom}} j_{j_{\xi}}(\mu) x_{\mu}:=x_{j_{\xi}} \neq 0 \in \mathbb{T}^{c}$. Naturalmente, esta condição vale sempre que $x_{j_{\xi}}(\xi) \neq 0$.

Suponhamos que $G$ é um grupo topológico infinito $T_{2}$, enumeravelmente compacto e sem seqüências não triviais convergentes. Pela proposição 4.1.4, dado que $G$ é em particular pseudocompacto e homogêneo, $|G| \geq \mathfrak{c}$. Note que qualquer subconjunto fechado em $G$, também deve ser de cardinalidade $\mathfrak{c}$. Como conseqüência disso, em principio podemos associar pontos de acumulação distintos a seqüências distintas. A idéia é garantir que $x_{\xi}$ será um ponto de acumulação da seqüência associada a $h_{\xi}$. Se mostrarmos que $X$ é livre, em particular obteríamos que esse conjunto está fielmente indexado mediante $\left\{x_{\xi}: \xi<\mathfrak{c}\right.$, e assim, de acordo com a cardinalidade do grupo, cada seqüência não trivial em $G$ teria exatamente c pontos de acumulação. Portanto, em cada etapa indutiva precisamos testemunhar que o elemento do grupo correspondente a $j_{\xi}, x_{j_{\xi}}$ é não nulo e, também, manter os pontos de acumulação que previamente temos associado. Notemos que de acordo com a codificação dos elementos de 
$G$ feita pelas funções $j_{\alpha}$, é possível separar os pontos de acumulação em dois tipos: temos um conjunto enumerável deles que depende de dom $j_{\xi}$ e os restantes que não dependem desse domínio.

Naturalmente, em cada etapa da indução o primeiro tipo de pontos irá crescendo, pelo qual resulta adequado definir indutivamente uma coleção de ordinais que guarde informação acerca dos domínios das funções das que os pontos de acumulação dependem, ou, em outras palavras, dos elementos do conjunto $X$ dos quais são combinações lineares. Com a seguinte definição, pretende-se que os pontos de acumulação cuja codificação depende do conjunto $\operatorname{dom} j_{\xi}$, estejam contidos em $S(\xi)$ :

$$
\begin{aligned}
& S(n)=\omega, \quad \text { se } n \in \omega \\
& S(\xi)=\{\xi\} \cup \bigcup\left\{S(\mu): \mu \in \bigcup_{n \in \omega} \operatorname{dom} h_{\xi}(n)\right\}, \quad \text { se } \xi \in[\omega, \mathfrak{c})
\end{aligned}
$$

Como é natural, se $F \subseteq \mathfrak{c}$, tomamos $S(F) \stackrel{\text { def }}{=} \bigcup_{\mu \in F} S(\mu)$.

Lema 2.2.15. Para cada $\xi \in[\omega, \mathfrak{c}), S(\xi)$ é enumerável; além disso, se $\mu \in S(\xi)$, então $\bigcup_{n \in \omega} \operatorname{dom} h_{\mu}(n) \subseteq S(\xi)$.

Demonstração. Por indução em c. Para cada $m \in \omega, S(m)=\omega$ que é enumerável. Como $h_{k}$ não está definido para $k<\omega$, a segunda asserção é satisfeita por vacuidade.

Suponha agora que $\xi \in[\omega, \mathfrak{c})$ e que para cada $\mu<\xi, S(\mu)$ é enumerável. Como $\operatorname{dom} h_{\xi}(n)$ é finito para cada $n \in \omega, \bigcup_{n \in \omega} h_{\xi}(n)$ é enumerável, $\{\xi\} \cup \bigcup\left\{S(\mu): \mu \in \bigcup_{n \in \omega} \operatorname{dom} h_{\xi}(n)\right\}=S(\xi)$ é também enumerável.

Seja $\mu \in S(\xi)$. De acordo com a definição, temos que $\mu=\xi$ ou $\mu \in S(v)$ para algum $v \in \bigcup_{n \in \omega} \operatorname{dom} h_{\S}(n)$. No primeiro caso, de acordo com a enumeração das seqüências, temos que $\bigcup_{n \in \omega} \operatorname{dom} h_{\xi}(n)=\bigcup_{n \in \omega} \operatorname{dom} h_{\mu}(n) \subseteq \xi$. No segundo caso, $v<\xi$ posto que $v \in \bigcup_{n \in \omega} \operatorname{dom} h_{\xi}(n)$; como $\mu \in S(v)$, por hipótese de indução $\bigcup_{n \in \omega} \operatorname{dom} h_{\mu}(n) \subseteq S(v) \subseteq S(\xi)$.

Voltando à construção indutiva, na etapa $\omega$ definimos para cada $n \in \omega, x_{n} \uparrow_{\omega} \in \mathbb{T}^{\omega}$ arbitrariamente. Suponha que na etapa $\alpha<\beta$ as seguintes condições indutivas são satisfeitas:

1. para cada $\xi<\alpha<\beta, x_{\xi} \uparrow_{\alpha}$ tem sido definido;

2. para cada $\xi \in[\omega, \alpha), \sum_{\mu \in \operatorname{dom}} j_{\xi \xi}(\mu) x_{\mu}(\xi) \neq 0$;

3. para cada $\xi \in[\omega, \alpha), x_{\xi} \uparrow_{\alpha}$ é um ponto de acumulação da seqüência associada a $h_{\S}$ em $\mathbb{T}^{\alpha}$. 
Vamos mostrar que para $\beta$ valem também essas condições; se $\beta$ for um ordinal limite, basta definir $x_{\xi} \uparrow_{\beta}=\bigcup_{\xi<\alpha<\beta} x_{\xi} \uparrow_{\alpha}$. Claramente, por se tratar de uma extensão, as condições 1) e 2) anteriores são válidas para $\beta$. Para ver que 3) também é satisfeita, seja $\xi \in[\omega, \beta)$. Por hipótese de indução, para cada $\alpha<\beta$ tal que $\xi<\alpha, x_{\xi} \uparrow_{\alpha}$ é ponto de acumulação da seqüência em $\mathbb{T}^{\alpha}$ associada a $h_{\xi}$; em virtude do lema $2.2, \quad x_{\xi} \uparrow_{\beta}$ é ponto de acumulação da seqüência associada a $h_{\S}$ em $\mathbb{T}^{\beta}$, isto é, $\beta$ satisfaz 3).

Agora suponha que $\beta=\alpha+1$ para algum $\alpha \leq \omega$. Como $\mathbb{T}^{\alpha}$ é compacto, podemos escolher um ponto de acumulação $y \in \mathbb{T}^{\alpha}$ da seqüência em $\mathbb{T}^{\alpha}$ associada a $h_{\alpha}$ :

$$
\left\{\sum_{\mu \in \operatorname{dom} h_{\alpha}(n)} h_{\alpha}(n)(\mu) x_{\mu} \uparrow_{\alpha}: n \in \omega\right\}
$$

Como na construção da seção anterior, tomamos $x_{\alpha} \uparrow_{\alpha} \stackrel{\text { def }}{=} y$. Também, de maneira similar mas

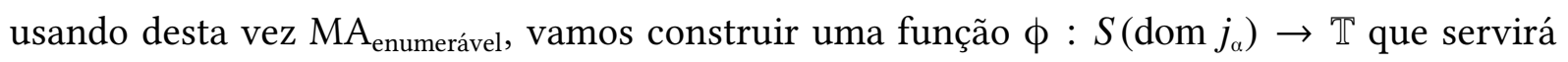
para definir $x_{\mu}(\alpha)$ para cada $\mu \in S\left(\operatorname{dom} j_{\alpha}\right)$.

Como antes, $\mathcal{B}$ representa uma base enumerável de $\mathbb{T}$ contendo, por conveniência ao próprio espaço $\mathbb{T}$ e $\|\cdot\|$ representa a norma euclidiana em $\mathbb{R}^{2}$ restrita ao subespaço $\mathbb{T}$.

Seja

$$
\mathbb{P} \stackrel{\text { def }}{=}\left\{f \in \operatorname{Fn}\left(S\left(\operatorname{dom} j_{\alpha}\right), \mathcal{B}\right): \operatorname{dom} j_{\alpha} \subseteq \operatorname{dom} f \text { e } 0 \notin \overline{\sum_{\mu \in \operatorname{dom} j_{\alpha}} j_{\alpha}(\mu) f(\mu)}\right\}
$$

Dados $f, g \in \mathbb{P}$, dizemos que $f \leq g$ se e somente se $\operatorname{dom} g \subseteq \operatorname{dom} f$ e para cada $\mu \in \operatorname{dom} g$, $f(\mu)=g(\mu)$ ou $\bar{f}(\mu) \subseteq g(\mu)$. Notemos que $S\left(\operatorname{dom} j_{\alpha}\right)=\bigcup_{\mu \in \operatorname{dom} f_{\alpha}} S(\mu)$ é um conjunto enumerável, dado que dom $j_{\alpha}$ é finito; portanto, a ordem $\mathbb{P}$ é enumerável.

Vamos definir certas coleções que permitem construir convenientemente conjuntos densos em $\mathbb{P}$.

Para cada $F \in[\alpha]^{<\omega}, n \in \omega$ e $\mu \in S\left(\operatorname{dom} j_{\alpha}\right)$, seja

$$
U(\mu, F, n)=\left\{f \in \mathbb{T}^{\alpha}: \forall \theta \in F\left\|f(\theta)-x_{\mu} \uparrow_{\alpha}(\theta)\right\|<\frac{1}{n+1}\right\}
$$

Codificaremos os pontos da seqüência associada a $h_{\mu}$ em $\mathbb{T}^{\alpha}$ que estão no aberto básico $U(\mu, F, n)$ mediante a seguinte coleção:

$$
E(\mu, F, n)=\left\{m \in \omega: \forall \theta \in F\left\|x_{h_{\mu}(m)}(\theta)-x_{\mu}(\theta)\right\|<\frac{1}{n+1}\right\}
$$

lembrando que temos de garantir que $x_{\mu}$ seja um ponto de acumulação da seqüência associada a $h_{\mu}$. 
Lema 2.2.16. Consideremos um conjunto de índices I e uma função $h: \omega \rightarrow \operatorname{Fn}(I, \mathbb{Z} \backslash\{0\})$ tal que para cada $n \in \omega, h(n) \neq \varnothing$. Se $U \subseteq \mathbb{T}$ é aberto, $A \in[\omega]^{\omega}$ e $f: I \rightarrow \mathcal{B}$ é uma função tal que o conjunto $\{i \in I: f(i) \neq \mathbb{T}\}$ é finito, então existem uma função $g: I \rightarrow \mathcal{B}$ e $m \in A$ satisfazendo

1. para cada $i \in I, \overline{g(i)} \subseteq \overline{f(i)}$;

2. o conjunto $\{i \in I: g(i) \neq \mathbb{T}\}$ é finito $e$,

3. $\sum_{i \in \operatorname{dom} h(m)} h(m)(i) g(i) \subseteq U$.

Lema 2.2.17. Para cada $F \in[\alpha]^{<\omega}, n, k \in \omega e \mu \in S$ (dom $j_{\alpha}$ ), o conjunto

$$
\begin{aligned}
& \mathbb{D}_{\mu, F, n, k}=\{f \in \mathbb{P}: \exists m \in E(\mu, F, n) \backslash k \text { tal que } \\
& \left.\qquad \operatorname{dom} h_{\mu}(m) \subseteq \operatorname{dom} f, \sum_{v \in \operatorname{dom} h_{\mu}(m)} h_{\mu}(m)(v) f(v) \subseteq f(\mu) \text { e } \operatorname{diam}(f(\mu))<\frac{1}{n+1}\right\}
\end{aligned}
$$

é denso em $\mathbb{P}$.

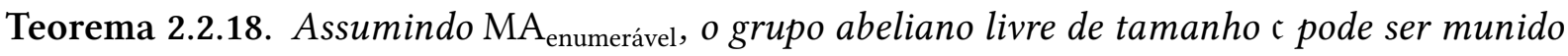
de uma topologia de grupo que faz dele um grupo de van Douwen.

Demonstração. Seja $\mathscr{E}=\left\{\mathbb{D}_{\mu, F, n, k}: \mu \in S\left(\operatorname{dom} j_{\alpha}\right), F \in[\alpha]^{<\omega}, k, n \in \omega\right\}$. Pelo lema anterior, cada um dos elementos de $\mathscr{E}$ é denso na ordem $\mathbb{P}$; dado que $|\mathscr{E}| \leq|\alpha|<\mathfrak{c}$, e $\mathbb{P}$ é enumerável, ao estarmos assumindo $M A_{\text {enumerável }}$ obtemos um filtro $\mathbb{G}$ em $\mathbb{P}$ genérico para esta coleção.

A partir do filtro $\mathbb{G}$, definimos a aplicação $\Phi: S\left(\operatorname{dom} j_{\alpha}\right) \rightarrow \wp(\mathcal{B})$ tomando para cada $\mu \in S\left(\operatorname{dom} j_{\alpha}\right)$

$$
\Phi(\mu)=\{f(\mu): \mu \in \operatorname{dom} f, f \in \mathbb{G}\}
$$

Em virtude da definição dos elementos da coleção $\mathscr{E}, \Phi(\mu) \neq \varnothing$ para cada $\mu \in S\left(\operatorname{dom} j_{\alpha}\right)$, logo $\bigcap_{V \in \Phi(\mu)} \bar{V} \neq \varnothing$. De fato, dados $f, g \in \mathbb{G}$, tais que $\mu \in \operatorname{dom} f \cap \operatorname{dom} g$ existe $r \in \mathbb{G}$ tal que $r \leq f, g$. Daí, $r(\mu) \subseteq f(\mu) \cap g(\mu)$ ou $\overline{r(\mu)} \subseteq f(\mu) \cap g(\mu)$; em qualquer caso, $f(\mu) \cap g(\mu) \neq \varnothing$ o que implica $\overline{f(\mu) \cap g(\mu)} \subseteq \overline{f(\mu)} \cap \overline{g(\mu)} \neq \varnothing$. Percebendo que $\Phi(\mu)$ é enumerável, pois $\mathbb{G}$ também é, e que os elementos de $\Phi(\mu)$ são subconjuntos do espaço $T_{2}$-compacto $\mathbb{T}$, obtemos $\bigcap_{V \in \Phi(\mu)} \bar{V}=\bigcap_{f(\mu) \in \Phi(\mu)} \overline{f(\mu)} \neq \varnothing$.

Em conseqüência, para cada $\mu \in S\left(\operatorname{dom} j_{\alpha}\right)$ podemos escolher $\phi_{\mathbb{G}}(\mu) \in \bigcap_{V \in \Phi(\mu)} \bar{V}$, obtendo desta maneira uma aplicação $\phi_{\mathbb{G}}: S\left(\operatorname{dom} j_{\alpha}\right) \rightarrow \mathbb{T}$.

Definimos para cada $\mu \in S\left(\operatorname{dom} j_{\alpha}\right), x_{\mu}(\alpha)=\phi_{\mathbb{G}}(\mu)$. Notemos que a condição 2$)$ é satisfeita para todo $\xi<\alpha$, logo resta provar que vale também para $\xi=\alpha$; de fato, para cada $f \in \mathbb{G}$, 
$\mu \in \operatorname{dom} f$ temos

$$
\sum_{\mu \in \operatorname{dom} j_{\alpha}} j_{\alpha}(\mu) x_{\mu}(\alpha)=\sum_{\mu \in \operatorname{dom} j_{\alpha}} j_{\alpha}(\mu) \phi_{G}(\mu) \in \sum_{\mu \in \operatorname{dom} j_{\alpha}} j_{\alpha}(\mu) \overline{f(\mu)} \subseteq \overline{\sum_{\mu \in \operatorname{dom} j_{\alpha}} j_{\alpha}(\mu) f(\mu)}
$$

Em virtude da definição de $\mathbb{P}, 0 \notin \overline{\sum_{\mu \in \operatorname{dom}} j_{j_{\alpha}}(\mu) f(\mu)}$; portanto, $\sum_{\mu \in \operatorname{dom} j_{\alpha}} j_{\alpha}(\mu) \phi_{G}(\mu) \neq 0$, ou seja, a condição 2) é satisfeita por $\beta$.

Resta verificar agora, que para cada $\xi \in[\omega, \alpha]$ as condições 1) e 3) são satisfeitas. Notemos que para $\xi \in S\left(\operatorname{dom} j_{\alpha}\right), \xi<\alpha$ já definimos $x_{\xi} \uparrow_{\beta}$, logo a condição 1) é satisfeita para estes pontos.

Indutivamente, iremos definir $x_{\xi} \uparrow_{\beta}$ para cada $\xi \in \beta \backslash S\left(\operatorname{dom} j_{\alpha}\right)$, mediante uma extensão da função $\phi_{G}$. Com efeito, suponhamos que para $\xi \in \beta \backslash S\left(\operatorname{dom} j_{\alpha}\right)$ os pontos do conjunto $S\left(\operatorname{dom} j_{\alpha}\right) \cup \xi$ satisfazem as condições 1$)$ e 3 ), que temos definido $\phi_{\mathbb{G}} \Gamma_{\xi}$ mas ainda $\phi_{\mathbb{G}}(\xi)$ não foi definido. Por hipótese, $x_{\xi} \uparrow_{\alpha}$ é um ponto de acumulação da seqüência em $\mathbb{T}^{\alpha}$ associada a $h_{\S}$ e $\phi(\mu)$ já foi definida para cada $\mu \in \bigcup_{n \in \omega} \operatorname{dom} h_{\xi}(n) \subseteq \xi$. Consideremos a seqüência em $\mathbb{T}$ dada por

$$
\left\{\sum_{\mu \in \operatorname{dom} h_{\xi}(n)} h_{\xi}(n)(\mu) \phi_{\mathbb{G}}(\mu): n \in \omega\right\}
$$

Como $\mathbb{T}$ é compacto, existe um ponto de acumulação $z \in \mathbb{T}$ da seqüência acima; daí, o ponto $x_{\xi} \Gamma_{\alpha}^{r} z$ é um ponto de acumulação da seqüência em $\mathbb{T}^{\beta}$ dada por

$$
\left\{\sum_{\mu \in \operatorname{dom} h_{\xi}(n)} h_{\xi}(n)(\mu) x_{\mu} \uparrow_{\alpha}^{\frown} \sum_{\mu \in \operatorname{dom} h_{\xi}(n)} h_{\xi}(n)(\mu) \phi_{G}(\mu): n \in \omega\right\}
$$

Em conseqüência, ao definir $\phi_{\mathbb{G}}(\alpha)=z$ e tomar $x_{\xi}(\alpha):=z$, temos que as hipóteses 1) e 3) são satisfeitas por $\xi$ na etapa $\beta=\alpha+1$ da indução.

Ao final do processo indutivo, para cada $\mu<\mathfrak{c}$ define-se

$$
x_{\mu} \stackrel{\text { def }}{=} \bigcup_{v \in(\mu, c)} x_{\mu} \uparrow_{v}
$$

Se $X=\left\{x_{\mu}: \mu<\mathfrak{c}\right\}$ e $G:=\langle X\rangle$, de acordo com as considerações feitas ao inicio, qualquer combinação linear não nula de elementos de $X$ está codificada por uma função $j_{\xi} \in$ $\operatorname{Fn}\left([\mathfrak{c}]^{<\omega}, \mathbb{Z} \backslash\{0\}\right)$ para algum ordinal $\xi \in[\omega, \mathfrak{c})$; como a condição 3) é satisfeita, $\sum_{\mu \in \operatorname{dom}} j_{\xi \xi}(\mu) x_{\mu}(\xi) \neq$ $0 \in \mathbb{T}$; logo, $\sum_{\mu \in \operatorname{dom}} j_{j_{\xi}}(\mu) x_{\mu}=x_{j_{\xi}} \neq 0 \in \mathbb{T}^{c}$, isto é, $G$ é uma realização do grupo abeliano livre de cardinalidade $\mathfrak{c}$ no grupo $\mathbb{T}^{\mathrm{c}}$.

Seja $\left\{z_{n}: n \in \omega\right\}$ uma seqüência em $G$; sem perda de generalidade, podemos supor que a 
seqüência é injetora e não contém entre os seus termos ao elemento $0 \in \mathbb{T}^{c}$; de acordo com a construção, existe $\zeta \in[\omega, \mathfrak{c})$ tal que para cada $n \in \omega, z_{n}=x_{h_{\zeta}(n)}$; em virtude da condição 3), , $x_{\zeta} \uparrow_{\alpha}$ é ponto de acumulação da seqüência $\left\{x_{h_{\zeta}(n)} \uparrow_{\alpha}: n \in \omega\right\}$ para cada $\alpha<\mathfrak{c}$, logo $x_{\zeta}$ é ponto de acumulação de $\left\{z_{n}: n \in \omega\right\}$. Portanto, $G$ é enumeravelmente compacto.

\subsubsection{Uma topologia de grupo e.c. sobre $\mathrm{A}\left(2^{\mathrm{c}}\right)$ via extensões genéricas}

Partindo do mesmo circulo de idéias da construção anterior, Koszmider, Tomita e Watson [46] mostraram, via forcing, que é possível construir uma topologia de grupo enumeravelmente compacta e sem sequências não triviais convergentes sobre o grupo abeliano livre com $2^{c}$ geradores. A seguir, detalharemos essa construção; convém dizer que a ordem utilizada neste exemplo tem certa similaridade com a anterior motivo pelo qual manteremos a notação ali introduzida.

\section{A ordem parcial}

Seja $\kappa$ um cardinal regular não enumerável satisfazendo $\kappa^{\omega}=\kappa$. Para codificar às seqüências injetoras do grupo que será construído, fixemos uma enumeração $\left\{h_{\xi}: \omega \leq \xi<\kappa\right\}$ de todas as funções $h \in \operatorname{Fn}(\kappa, \mathbb{Z} \backslash\{0\})^{\omega}$ onde $\operatorname{dom} h \neq \varnothing$ para cada $n \in \omega$. Adicionalmente, suponhamos que $\bigcup_{n \in \omega} h_{\xi} \subseteq \xi$ para cada $\xi \in[\omega, \kappa)$.

Seja

$$
S(\xi)= \begin{cases}\omega, & \text { se } \xi \in \omega \\ \{\xi\} \cup \bigcup\left\{S(\mu): \mu \in \bigcup_{n \in \omega} \operatorname{dom} h_{\xi}(n)\right\}, & \text { se } \xi \in[\omega, \kappa)\end{cases}
$$

Definição 2.2.19. Seja $\mathbb{P}$ a noção de forcing formada pelas condições $p=\left(\alpha_{p}, D_{p}, f_{p}\right)$ onde:

1. $\alpha_{p} \in \omega_{1}$

2. $D_{p} \in[\kappa]^{\omega}$ e $D_{p} \supset \bigcup_{\xi \in D_{p}} S(\xi)$

3. $f_{p}: D_{p} \rightarrow \mathbb{T}^{\alpha_{p}}$;

4. para cada $\xi \in D_{p} \backslash \omega, f_{p}(\xi)$ é um ponto de acumulação da seqüência

$$
\left\{\sum_{\mu \in \operatorname{dom} h_{\xi}(n)} h_{\xi}(n)(\mu) f_{p}(\mu): n \in \omega\right\}
$$

5. o conjunto $\left\{f_{p}(\xi): \xi \in D_{p}\right\}$ é um conjunto livre, isto é, para cada função $j \in \operatorname{Fn}\left(D_{p}, \mathbb{Z} \backslash\{0\}\right)$, temos que $\sum_{\xi \in \operatorname{dom} j} j(\xi) f_{p}(\xi) \neq 0 \in \mathbb{T}^{\alpha_{p}} ;$ 
$\mathbb{P}$ será ordenada pela relação $\leq$, onde $p \leq q$ se e somente se $\alpha_{q} \leq \alpha_{p}, D_{q} \subseteq D_{p}$ e para cada $\xi \in D_{q}, f_{p}(\xi) \uparrow_{\alpha_{q}}=f_{q}(\xi)$.

Para provar que a noção de forcing é "boa" o suficiente para nossa construção temos o seguinte resultado:

Lema 2.2.20. Sejam $D \in[\kappa]^{\omega}, \beta<\omega_{1}$ e $f: D \rightarrow \mathbb{T}^{\beta}$ tais que $f(\xi)$ é o limite da seqüência $\left\{\sum_{\mu \in \operatorname{dom} h_{\xi}(n)} h_{\xi}(n)(\mu) f(\mu): n \in A_{\xi}\right\}$ onde para cada $\xi \in D \backslash \omega, A_{\xi} \in[\omega]^{\omega}$. Se $j \in \operatorname{Fn}(D, \mathbb{Z} \backslash\{0\}) e$ $F=\operatorname{dom} j$, então existe uma função $\phi: D \rightarrow \mathbb{T}$ tal que $\sum_{\mu \in F} j(\mu) \phi(\mu) \neq 0 \in \mathbb{T}$ e para cada $\xi \in D \backslash \omega, \phi(\xi)$ é o limite de uma subseqüência de $\left\{\sum_{\mu \in \operatorname{dom} h_{\xi}(n)} h_{\xi}(n)(\mu) \phi(\mu): n \in A_{\xi}\right\}$.

Demonstração. Observemos que $w\left(\mathbb{T}^{\beta}\right)=\boldsymbol{\aleph}_{0}$, posto que $\mathbb{T}$ é de peso enumerável e $\beta<\omega_{1}$; assim, fixemos uma base enumerável $\mathcal{B}^{*}$ do espaço $\mathbb{T}^{\beta}$. Seja

$$
\mathbb{M} \stackrel{\text { def }}{=}\left\{g \in \operatorname{Fn}\left(D, \mathcal{B}^{*}\right): F \subseteq \operatorname{dom} g \text { e } 0 \notin \overline{\sum_{\mu \in F} j(\mu) g(\mu)}\right\}
$$

Em $\mathbb{M}$, dizemos que $g \leq h$ se e somente se $\operatorname{dom} h \subseteq \operatorname{dom} g$ e para cada $\mu \in \operatorname{dom} h, g(\mu)=h(\mu)$ ou $\overline{g(\mu)} \subseteq h(\mu)$. Naturalmente, ( $\mathbb{M}, \leq)$ constitui uma ordem parcial enumerável.

Como no parágrafo anterior, definiremos certas coleções para construir conjuntos densos na ordem $\mathbb{M}$. Para cada $L \in[\beta]^{<\omega}, n \in \omega$ e $\xi \in D$, seja

$$
E(\xi, L, n)=\left\{m \in \omega: \forall \theta \in L \underset{\mu \in \operatorname{dom} h_{\xi}(m)}{ } h_{\xi}(m)(\mu) f(\mu)(\theta)-f(\xi)(\theta) \|<\frac{1}{n+1}\right\}
$$

Nessa situação, seja

$$
\begin{aligned}
& \mathbb{D}_{\xi, L, n, k}=\{g \in \mathbb{M}: \exists m \in E(\xi, L, n) \backslash k \text { tal que } \\
& \left.\qquad \operatorname{dom} h_{\xi}(m) \subseteq \operatorname{dom} g, \sum_{v \in \operatorname{dom} h_{\xi}(m)} h_{\S}(m)(v) g(v) \subseteq g(\xi) \text { e } \operatorname{diam}(g(\xi))<\frac{1}{n+1}\right\}
\end{aligned}
$$

Aplicando um argumento similar ao do lema 2.2.11, temos que o conjunto anterior é denso em $\mathbb{M}$. Observe a coleção $\mathscr{D}=\left\{\mathbb{D}_{\xi, L, n, k}: L \in[\beta]^{<\omega}, n, k \in \omega, \xi \in D\right\}$ tem cardinalidade $\boldsymbol{\aleph}_{0}$. Como MA( $\omega)$ é verdadeiro em ZFC, e a coleção $\mathscr{D}$ é enumerável, obtemos um filtro $\mathscr{D}$-genérico $\mathbb{G} \subseteq \mathbb{M}$. A partir desse filtro, definimos a aplicação $\Psi: D \rightarrow \wp\left(\mathcal{B}^{*}\right)$ tomando para cada $\xi \in D$,

$$
\Psi(\xi)=\{q(\xi): \xi \in \operatorname{dom} q, q \in \mathbb{G}\}
$$

Posteriormente, definimos $\phi: D \rightarrow \mathbb{T}$ de maneira que para cada $\xi \in D, \phi(\xi) \in \bigcap_{V \in \Psi(\xi)} \bar{V}$. De acordo com a definição da família $\mathcal{D}$, naturalmente $\phi$ satisfaz as condições do lema. 
O seguinte lema mostra que $\mathbb{P}$ é realmente uma noção de forcing que satisfaz propriedades "adequadas".

Lema 2.2.21. $\mathbb{P}$ é uma ordem parcial e satisfaz as seguintes propriedades:
a. P é enumeravelmente fechada;
b. assumindo $C H, \mathbb{P} e ́ \omega_{2}-c c$;
c. para cada $\xi<\kappa$, o conjunto $\mathcal{D}_{\xi}=\left\{p \in \mathbb{P}: \xi \in D_{p}\right\}$ é aberto e denso em $\mathbb{P}$;
d. para cada $\alpha<\omega_{1}$, o conjunto $\mathcal{H}_{\alpha}=\left\{p \in \mathbb{P}: \alpha_{p} \geq \alpha\right\}$ é aberto e denso em $\mathbb{P}$.

Demonstração. a. Seja $\left\{p_{n}: n \in \omega\right\}$ uma seqüência decrescente em $\mathbb{P}$, onde, para cada $n \in \omega$ temos $p_{n}=\left(\alpha_{n}, D_{n}, f_{n}\right)$. Definimos

$$
q=(\alpha, D, F)
$$

onde $\alpha:=\bigcup_{n \in \omega} \alpha_{n}, D:=\bigcup_{n \in \omega} D_{n}$ e $F:=\bigcup_{n \in \omega} f_{n}: D \rightarrow \mathbb{T}^{\alpha}$.

Afirmamos que $q \leq p_{n}$ para todo $n \in \omega$; note-se que $\alpha_{n}<\omega_{1}$, para cada $n \in \omega$; daí, já que $\omega_{1}$ é regular assumindo ZFC obtemos $\alpha=\bigcup_{n \in \omega} \alpha_{n} \in \omega_{1}$.

Dado que cada $D_{n} \subseteq \kappa$ é enumerável, a coleção $D=\bigcup_{n \in \omega} D_{n} \subseteq \kappa$ também é enumerável.

Finalmente $F$ constitui uma função, posto que a seqüência $\left\{f_{n}: n \in \omega\right\}$ é decrescente; vejamos que $F$ satisfaz as condições próprias da ordem $\mathbb{P}$. Observemos que

$$
\begin{aligned}
D=\bigcup_{n \in \omega} D_{n} & \supseteq \bigcup_{n \in \omega} \bigcup_{\xi \in D_{n}} S(\xi) \\
& =\bigcup_{\xi \in \bigcup D_{n}} S(\xi)=\bigcup_{\xi \in D} S(\xi)
\end{aligned}
$$

logo $F$ satisfaz a condição 1). Agora, seja $\xi \in D \backslash \omega$. Tomando $n_{0}=\min \left\{n \in \omega: \xi \in D_{n}\right\}$ temos que para cada $n \geq n_{0}, f_{n}(\xi)$ é ponto de acumulação da seqüência

$$
\left\{\sum_{\mu \in \operatorname{dom} h_{\xi}(k)} h_{\xi}(k)(\mu) f_{n}(\mu): k \in \omega\right\}=\left\{\sum_{\mu \in \operatorname{dom} h_{\xi}(k)} h_{\xi}(k)(\mu) F \uparrow_{\alpha_{n}}(\mu): k \in \omega\right\} \subseteq \mathbb{T}^{\alpha_{n}}
$$

De acordo com a proposição 2.2.6, $\xi$ constitui um ponto de acumulação da seqüência

$$
\left\{\sum_{\mu \in \operatorname{dom} h_{\xi}(k)} h_{\xi}(k)(\mu) F(\mu): k \in \omega\right\} \subseteq \mathbb{T}^{\alpha}
$$


Agora, seja $j \in \operatorname{Fn}(D, \mathbb{Z} \backslash\{0\}) \backslash\{\varnothing\}$. Como dom $j$ é finito, existe $n_{0}=\min \{n \in \omega: \operatorname{dom} j \subseteq$ $\left.D_{n}\right\}$. Visto que $\left\{f_{n}(\xi): \xi \in D_{n}\right\}$ é livre para cada $n \geq n_{0}$, temos

$$
\sum_{\xi \in \operatorname{dom} j} j(\xi) f_{n}(\xi)=\sum_{\xi \in \operatorname{dom} j} j(\xi) F \uparrow_{\alpha_{n}}(\xi) \neq 0
$$

Assim, $\sum_{\xi \in \operatorname{dom} j} j(\xi) F(\xi) \neq 0$ ou seja, a família $\{F(\xi): \xi \in D\}$ é livre.

Em virtude dos argumentos anteriores, $q \in \mathbb{P}$ e $q \leq p_{n}$ para cada $n \in \omega$, ou seja, $\mathbb{P}$ é enumeravelmente fechada.

b. Suponhamos que $\left\{p_{\eta}: \eta<\omega_{2}\right\}$ é uma anticadeia em $\mathbb{P}$, onde $p_{\eta}=\left(\alpha_{\eta}, D_{\eta}, f_{\eta}\right)$ para cada $\eta<\omega_{2}$. Seja $\mathcal{A}=\left\{D_{\eta}: \eta<\omega_{2}\right\}$; observemos que para cada $\eta$, temos $\left|D_{\eta}\right|=\omega<\omega_{1}$ e sob $\mathrm{CH}$, se $\alpha<\omega_{2},\left|\alpha^{<\omega_{1}}\right| \leq \omega_{1}<\omega_{2}$. Pelo lema do $\Delta$-sistema, existem $I \in\left[\omega_{2}\right]^{\omega_{2}}$ e $R \in[\kappa]^{\leq \omega}$ tais que para cada par de elementos distintos $\eta, \theta \in I, D_{\eta} \cap D_{\theta}=R$. Sendo $\left\{p_{\eta}: \eta \in \mathcal{B}\right\}$ uma anticadeia, temos que $R \neq \varnothing$. De outra parte, observando que para cada $\eta \in I, \alpha_{\eta}<\omega_{1}=\mathfrak{c}$, passando tal vez a um subconjunto de $I$ cardinalidade $\omega_{2}$, podemos assumir sem perda de generalidade que existe $\alpha<\mathfrak{c}$ tal que $\alpha_{\eta}=\alpha$ para todo $\eta \in I$. Sejam $\eta, \theta \in I$ distintos; como $p_{\eta}$ e $p_{\theta}$ são incompatíveis e $\alpha_{\eta}=\alpha_{\theta}$, existe $r \in R$ de maneira que $f_{\eta}(r) \neq f_{\theta}(r) \in \mathbb{T}^{\alpha}$. Portanto, a aplicação

$$
\begin{aligned}
\psi: \quad I & \rightarrow\left(\mathbb{T}^{\alpha}\right)^{R} \\
\eta & \mapsto f_{\eta} \uparrow_{R}
\end{aligned}
$$

é injetora, e, assim $|I| \leq\left|\left(\mathbb{T}^{\alpha}\right)^{R}\right| \leq \mathfrak{c}=\omega_{1}<\omega_{2}$, uma contradição. Em conseqüência, as anticadeias em $\mathbb{P}$ são de cardinalidade menor do que $\omega_{2}$, isto é, $\mathbb{P}$ é $\omega_{2}$-cc.

c. Fixemos $\xi<\kappa$. Seja $p \in \mathbb{P}$ tal que $\xi \notin D_{p}$, pois no caso contrário não temos nada que provar. Podemos achar um subconjunto $D \in[\kappa]^{\omega}$ tal que $D_{p} \cup\{\xi\} \subseteq D$ e $\bigcup_{\mu \in D} S(\mu) \subseteq D$, posto que $S(\xi)$ é enumerável. Vamos definir uma função $f: D \rightarrow \mathbb{T}^{\alpha_{p}}$ por indução em $D$. Naturalmente, tomamos $f \uparrow_{D_{p}} \stackrel{\text { def }}{=} f_{p}$. Seja agora $\eta \in D \backslash D_{p}$ é o menor elemento de $D$ para o qual $f(\eta)$ ainda não foi definido. Dado que $\bigcup_{n \in \omega} \operatorname{dom} h_{\eta}(n) \subseteq \eta$, temos já definido a seqüência

$$
\left\{\sum_{\theta \in \operatorname{dom} h_{\eta}(n)} h_{\eta}(n)(\theta) f(\theta): n \in \omega\right\}
$$

de maneira que podemos tomar um ponto de acumulação $f(\eta) \in \mathbb{T}^{\alpha_{p}}$ dela. Aplicando este argumento indutivamente, definimos $f$ sobre o conjunto $D$. De acordo com o anterior, temos que $D$ e $f$ satisfazem as condições 1) e 2) da noção de forcing $\mathbb{P}$. Seja $\left\{j_{n}: n \in \omega\right\}$ uma 
enumeração de $\operatorname{Fn}(D, \mathbb{Z} \backslash\{0\})$. Como $\alpha_{p}$ é enumerável temos que $\mathbb{T}^{\alpha_{p}}$ é seqüencialmente compacto, logo para cada $\xi \in D \backslash \omega$, existe $A_{\xi, 0} \in[\omega]^{\omega}$ tal que $f(\xi)$ é o limite da seqüência

$$
\left\{\sum_{\theta \in \operatorname{dom} h_{\xi}(n)} h_{\xi}(n)(\theta) f(\theta): n \in A_{\xi, 0}\right\}
$$

Aplicando o lema 2.2.20 no caso $j=j_{1}$, existe $\phi_{1}: D \rightarrow \mathbb{T}$ tal que para cada $\xi \in D \backslash \omega, \phi_{1}(\xi)$ é o limite da seqüência

$$
\left\{\sum_{\theta \in \operatorname{dom} h_{\xi}(n)} h_{\S}(n)(\theta) \phi_{1}(\theta): n \in A_{\xi, 1}\right\}
$$

sendo $A_{\xi, 1} \subseteq A_{\xi, 0}$ e $\sum_{\theta \in F_{1}} j(\theta) \phi_{1}(\theta) \neq 0$, onde $F_{1}=\operatorname{dom} j_{1}$. Ao aplicar esse lema indutivamente, onde na etapa $n+1$-ésima consideramos para cada $\xi \in D \backslash \omega$ a seqüência convergente a $f(\xi)$ dada por $\left\{\sum_{\theta \in \operatorname{dom} h_{\xi}(n)} h_{\xi}(n)(\theta) f(\theta): n \in A_{\xi, n}\right\}$ e a função $j_{n+1}$, obtemos para cada $\xi \in D \backslash \omega$ uma seqüência $\subseteq$-decrescente $\left\{A_{\xi, n}: n \in \omega\right\}$ tal que $\sum_{\theta \in F_{n}} j_{n}(\theta) \phi_{n}(\theta) \neq 0$ e

$$
\phi_{n}(\xi)=\lim _{k \in A_{\xi, n}} \sum_{\theta \in \operatorname{dom} h_{\xi}(k)} h_{\xi}(k)(\theta) \phi_{n}(\theta)
$$

Seja $\alpha_{q} \stackrel{\text { def }}{=} \alpha_{p}+\omega ;$ definimos $f_{q}: D \rightarrow \mathbb{T}^{\alpha_{q}}$ tomando para cada $\xi \in D$

$$
f_{q}(\xi)(\mu)= \begin{cases}f(\xi)(\mu), & \text { se } \mu \in \alpha \\ \phi_{n}(\xi), & \text { se } \mu=\alpha+n, n \in \omega \text { e } \xi \in D \backslash \omega\end{cases}
$$

Se tomarmos $q=\left(\alpha_{q}, D, f_{q}\right)$, pela construção obtemos que $q \in \mathcal{D}_{\xi} \subseteq \mathbb{P}$, e $q \leq p$.

Se $p \in D_{\xi}$ e $q \leq p$, dado que $D_{p} \subseteq D_{q}$ obtemos $\xi \in D_{q}$, isto é, $q \in D_{\xi}$. Portanto, $D_{\xi}$ é aberto em $\mathbb{P}$.

d. Fixemos $\alpha<\omega_{1}$ e seja $p \in \mathbb{P}$ tal que $\alpha_{p}<\alpha$. Definimos $f: D_{p} \rightarrow \mathbb{T}^{\alpha}$ tomando para cada $\xi \in D_{p}$,

$$
f(\xi)(\mu)= \begin{cases}f_{p}(\xi)(\mu), & \text { se } \mu \in \alpha_{p} \\ 0, & \text { se } \mu \in\left[\alpha_{p}, \alpha\right)\end{cases}
$$

Se $q \stackrel{\text { def }}{=}\left(\alpha, D_{p}, f\right)$, temos que $q \in \mathcal{H}_{\alpha} \subseteq \mathbb{P}$ e $q \leq p$.

Suponhamos agora que $p \in \mathcal{H}_{\alpha}$ e $q \leq p$. Como $\alpha \leq \alpha_{p} \leq \alpha_{q}$ temos $q \in \mathcal{H}_{\alpha}$, logo $\mathcal{H}_{\alpha}$ é aberto em $\mathbb{P}$.

De acordo com as propriedades da noção de forcing $\mathbb{P}$ obtém-se o principal teorema desta seção: 
Teorema 2.2.22. Sejam $\kappa$ um cardinal regular $e \mathbb{V} u m$ modelo de $\mathrm{GCH}+\kappa>\omega_{1}$. Se $\mathbb{G}$ é um filtro $\mathbb{P}$-genérico, então em $\mathbb{V}[\mathbb{G}]$ o grupo abeliano livre $H$ de cardinalidade $\kappa=\left(2^{\mathrm{c}}\right)^{\mathbb{V}[\mathbb{G}]}$ pode ser munido de uma topologia de grupo que faz dele um grupo de van Douwen.

Demonstração. Como a noção de forcing $\mathbb{P}$ é enumeravelmente fechada e $\omega_{2}$-c.c., temos que preserva cofinalidades e assim cardinalidades, logo na extensão $\mathbb{V}[\mathbb{G}]$, temos que $\omega_{1}<\kappa=$ $2^{\omega_{1}}$, sendo que em $\mathbb{V}[\mathbb{G}]$ os conjuntos enumeráveis são exatamente os conjuntos enumeráveis no modelo base $\mathbb{V}$.

Para cada $\alpha<2^{\text {c }}$ definimos

$$
x_{\alpha}=\bigcup\left\{f_{p}(\alpha): p \in \mathbb{G} \text { e } \alpha \in D_{p}\right\}
$$

Visto que as famílias $\left\{\mathcal{D}_{\xi}: \xi<\kappa\right\}$ e $\left\{\mathcal{H}_{\mu}: \mu<\mathfrak{c}\right\}$ estão formadas por subconjuntos densos em $\mathbb{P}$, para cada $\alpha \in \kappa=2^{c}$, $x_{\alpha}$ é uma função cujo domínio é $2^{\mathfrak{c}}$ e cuja imagem é um subconjunto de $\mathbb{T}^{\mathrm{c}}$.

Afirmamos que o conjunto $X=\left\{x_{\alpha}: \alpha<2^{c}\right\}$ é livre. Para provar isto, seja $j \in$ $\operatorname{Fn}\left(2^{c}, \mathbb{Z} \backslash\{0\}\right)$ uma função distinta de vazio; como cada elemento de $\mathbb{P}$ guarda na segunda coordenada um subconjunto enumerável de $2^{c}$ (que constitui o domínio da função a ele associada) e $\mathbb{G}$ é $\mathbb{P}$-genérico, podemos tomar $p \in \mathbb{G}$ de maneira que dom $j \subseteq D_{p}$. Pela condição 3) da ordem temos que

$$
\sum_{\xi \in \operatorname{dom} j} j(\xi) f_{p}(\xi)=\sum_{\xi \in \operatorname{dom} j} j(\xi) x_{\xi} \uparrow_{\alpha_{p}} \neq 0 \in \mathbb{T}^{2^{\alpha_{p}}}
$$

logo existe $\beta<\alpha_{p}$ satisfazendo $\sum_{\xi \in \operatorname{dom} j} j(\xi) x_{\xi}(\beta) \neq 0 \in \mathbb{T}$. Assim, $X$ constitui um conjunto livre, dado que $\operatorname{Fn}\left(2^{\mathfrak{c}}, \mathbb{Z} \backslash\{0\}\right) \backslash\{\varnothing\}$ codifica todas as combinações lineares não triviais de elementos desse conjunto.

Para mostrarmos que o grupo $\langle X\rangle$ é enumeravelmente compacto, consideremos uma sequência $\left\{y_{n}: n \in \omega\right\}$ em $G$; basta supor que dita seqüência é injetora e que não contém o elemento $0 \in G$. Daí, existe uma função injetora $h: \omega \rightarrow \operatorname{Fn}\left(2^{c}, \mathbb{Z} \backslash\{0\}\right)$ tal que para cada $n \in \omega$,

$$
y_{n}=\sum_{\mu \in \operatorname{dom} h(n)} h(n)(\mu) x_{\mu}=x_{h(n)}
$$

Como em $\mathbb{V}[\mathbb{G}]$ não temos subconjuntos enumeráveis além dos que tínhamos no modelo $\mathbb{V}$, $h$ está em $\mathbb{V}$, existe $\beta \in\left[\omega, 2^{c}\right)$ tal que $h_{\beta}=h$. Como $\mathcal{D}_{\beta}$ é denso em $\mathbb{P}$, existe $p \in \mathbb{G}$ tal que $\beta \in D_{p}$. Adicionalmente, dado $\alpha<\mathfrak{c}$, levando em consideração que $\mathcal{H}_{\alpha}$ é denso, podemos 
assumir também que $\alpha_{p}>\alpha$. Em virtude da definição de $\mathbb{P}, f_{p}(\beta) \uparrow_{\alpha}=x_{\beta} \uparrow_{\alpha}$ é ponto de acumulação da seqüência

$$
\begin{aligned}
\left\{\sum_{\mu \in \operatorname{dom} h_{\beta}(n)} h_{\beta}(n)(\mu) f_{p}(\mu) \uparrow_{\alpha}: n \in \omega\right\} & =\left\{\sum_{\mu \in \operatorname{dom} h(n)} h(n)(\mu) x_{\mu} \uparrow_{\alpha}: n \in \omega\right\} \\
& =\left\{x_{h(n)} \uparrow_{\alpha}: n \in \omega\right\} \\
& =\left\{y_{n} \uparrow_{\alpha}: n \in \omega\right\}
\end{aligned}
$$

Como isto ocorre para cada $\alpha<\mathfrak{c}$, e em $\mathbb{V}[\mathbb{G}]$ o ordinal $\omega_{1}=\mathfrak{c}$ é limite, em virtude da proposição 2.2.6, $x_{\beta}$ é ponto de acumulação da seqüência $\left\{y_{n}: n \in \omega\right\} \subseteq\left(\mathbb{T}^{c}\right)^{2^{c}}$. Portanto, $G$ é enumeravelmente compacto. 



\section{CAPÍTULO 3}

\section{Topologias de grupo e ultrafiltros seletivos}

Neste capítulo vamos estudar construções de grupos de van Douwen feitas usando ultrafiltros seletivos. Por conveniência, dedicamos uma seção deste capítulo ao estudo da $p$ compacidade, dada a dependência das construções referidas nesta seção com este conceito.

De maneira similar às outras construções, usando esta técnica, os grupos são construídos definindo coordenadas por meio de homomorfismos num grupo compacto, porém, em cada etapa ditos homomorfismos são construídos de maneira independente.

\subsection{Espaços $p$-compactos}

\section{$\beta \omega, P$-pontos e ultrafiltros seletivos}

Definição 3.1.1. Um conjunto $\mathfrak{F} \subseteq \wp(\omega)$ é dito um filtro sobre $\omega$ se

1. $\omega \in \mathfrak{F}$ e $\varnothing \notin \mathfrak{F}$

2. se $X \in \mathfrak{F}$ e $Y \in \mathfrak{F}$, então $X \cap Y \in \mathfrak{F}$

3. se $X \in \mathfrak{F}$ e $X \subseteq Y$, então $Y \in \mathfrak{F}$

Uma base para um filtro $\mathfrak{F}$ sobre $\omega$ é uma família não vazia $\mathfrak{B} \subseteq \wp(\omega)$ tal que

1. $\varnothing \notin \mathfrak{B}$

2. se $A, B \in \mathfrak{B}$ então existe $C \in \mathfrak{B}$ tal que $C \subseteq A \cap B$

Nesse caso, o conjunto $\langle\mathfrak{B}\rangle=\{X \subseteq \omega: \exists A \in \mathfrak{B}$ tal que $A \subseteq X\}$ é um filtro sobre $\omega$ e denomina-se filtro gerado por $\mathfrak{B}$. 
Um filtro $\mathfrak{F}$ sobre $\omega$ é principal se possui uma base formada por um único elemento; caso contrário, o filtro $\mathfrak{F}$ é denominado livre.

Definição 3.1.2. Um filtro $u$ sobre $\omega$ é um ultrafiltro se é maximal com respeito à inclusão. De maneira alternativa, $u$ é um ultrafiltro se para cada $X \subseteq \omega, X \in u$ ou $\omega \backslash X \in u$.

A compactificação de Čech-Stone de $\omega$, denotada por $\beta \omega$, é o único espaço compacto (a menos de homeomorfismo) contendo o espaço discreto $\omega$ como subespaço denso tal que para cada espaço compacto $K$ qualquer função $f$ de $\omega$ em $K$, admite uma extensão contínua $\beta f: \beta \omega \rightarrow K$.

Identificaremos $\beta \omega$ com o espaço que tem por suporte o conjunto dos ultrafiltros sobre $\omega$, cuja topologia é gerada pela base $\mathscr{B}=\{\widehat{A}: A \subseteq \omega\}$, onde $\widehat{A} \stackrel{\text { def }}{=}\{p \in \beta \omega: A \in p\}$. Neste espaço, $\omega$ é homeomorfo ao subespaço formado pelos ultrafiltros principais sobre $\omega$, enquanto que os pontos do resíduo $\omega^{*}=\beta \omega \backslash \omega$ correspondem aos ultrafiltros livres sobre $\omega$.

$\beta \omega$ constitui um dos espaços mais interessantes em topologia geral. Várias das propriedades topológicas dele são ainda desconhecidas, e não sabemos muito sobre os conceitos topológicos que podemos definir mediante os seus pontos, entre os quais se encontra a $p$ compacidade.

\section{A ordem de Rudin-Keisler $\leq_{R K}$}

Seja $f \in \omega^{\omega} \backslash\{\varnothing\}$ e $p, q \in \beta \omega$. É fácil ver que $\mathcal{B}=\{f[U]: U \in p\}$ satisfaz a propriedade de interseção finita pelo que constitui uma base de filtro; de fato, o objeto gerado por $\mathcal{B}$ é um ultrafiltro que corresponde justamente a $\beta f(p)$. Naturalmente, temos que

$$
\beta f(p)=q \leftrightarrow(\forall U \in p)(f[U] \in q) \leftrightarrow(\forall V \in q)\left(f^{-1}[V] \in p\right)
$$

Baseando-nos na seguinte pré-ordem, podemos definir vários tipos de elementos de $\omega$.

Definição 3.1.3. Dados $p, q \in \omega^{*}$, dizemos que $p \leq_{\mathrm{RK}} q$ se existe uma função $f: \omega \rightarrow \omega$ tal que $\beta f(q)=p$. : A pré-ordem $\leq_{\mathrm{RK}}$ definida sobre $\omega^{*}$ chama-se de ordem de Rudin-Keisler.

Como é natural, definindo $p \approx_{R K} q \leftrightarrow\left(p \leq_{\mathrm{RK}} q\right.$ e $\left.q \leq_{\mathrm{RK}} p\right)$, obtemos uma relação de equivalência sobre $\beta \omega$, logo, podemos dizer que dois ultrafiltros $p$ e $q$ são Rudin-Keisler equivalentes se e somente se existe uma permutação $\sigma$ de $\omega$ tal que $\beta \sigma(p)=q$.

Definição 3.1.4. Se $p \in \omega^{*}$, dizemos que $p$ é seletivo se for minimal na ordem de Rudin-Keisler. 
A continuação, listamos uma série de equivalências que fornecem diversas caracterizações dos ultrafiltros seletivos. Remetemos o leitor interessado na prova do seguinte teorema ao trabalho de Comfort e Negrepontis[19].

Teorema 3.1.5. Seja $p \in \omega^{*}$. As seguintes afirmações são equivalentes:

i. $p$ é seletivo;

ii. para cada $f: \omega \rightarrow \omega$ existe $A \in p$ tal que $f \uparrow_{A}$ é constante ou injetora;

iii. se $\left\{P_{n}: n \in \omega\right\}$ é uma partição de $\omega$, então existe $m \in \omega$ tal que $P_{m} \in p$ ou existe $B \in p$ tal que $\left|B \cap P_{n}\right|=1$ para cada $n \in \omega$.

iv. $p$ é fracamente Ramsey, isto é, para cada coloração $g:[\omega]^{2} \rightarrow\{0,1\}$, existe $C \in p$ tal que $C$ é g-homogêneo ${ }^{1}$;

v. para cada partição $P_{0} \cup P_{1}=[\omega]^{2}$, existe $D \in p$ e $j \in\{0,1\}$ tal que $[D]^{2} \subseteq P_{j}$.

Definição 3.1.6. Um ultrafiltro $p \in \omega^{*}$ é chamado de $P$-ponto se para qualquer partição $\left\{A_{n}\right.$ : $n \in \omega\}$ de $\omega$, existe $n \in \omega$ tal que $A_{n} \in p$ ou existe $A \in p$ tal que para todo $n \in \omega,\left|A \cap A_{n}\right|<\omega$.

Uma descrição combinatória equivalente à anterior definição é: $p$ é um $P$-ponto se e somente se para qualquer família $\left\{U_{n}: n \in \omega\right\} \subseteq p$, existe $U \in p$ tal que $\left|U_{n} \backslash U\right|<\omega$ para cada $n \in \omega$.

Pode-se provar que a coleção dos $P$-pontos em $\omega^{*}$ é um conjunto dirigido considerando a ordem de Rudin-Keisler. Observamos também que o item iii) do teorema anterior mostra que todo ultrafiltro seletivo é em particular um $P$-ponto; naturalmente, a recíproca dessa afirmação não é verdadeira.

A existência de ultrafiltros seletivos é independente da axiomática ZFC. De uma parte, assumindo $\mathrm{CH}$ ou MA, foi demonstrado que existem $2^{\mathrm{c}}$ ultrafiltros seletivos, entanto Shelah [57], via forcing, construiu um modelo sem $P$-pontos e em particular, sem ultrafiltros seletivos. Também, tem-se resultados de Kunen a este respeito, pois mostrou que no modelo real aleatório não existem ultrafiltros seletivos.

\section{O conceito de $p$-compacidade}

Definição 3.1.7. Se $p \in \omega^{*}$ e $f: \omega \rightarrow X$ é uma seqüência sobre um espaço de Tychonoff X, dizemos que o ponto $x \in X$ é um $p$-limite da seqüência $f$ se

\footnotetext{
${ }^{1}$ Um conjunto $C$ diz-se $g$-homogêneo se existe $j \in\{0,1\}$ tal que $g(\{a, b\})=j$ para cada par $\{a, b\} \in[C]^{2}$.
} 


$$
\beta f(p)=x
$$

onde $\beta f: \beta \omega \rightarrow \beta X$ representa a extensão de Čech-Stone de $f$. Nesse caso, escrevemos

$$
x=p-\lim _{n \in \omega} f_{n}
$$

Produto das definições, é fácil ver que $x=p-\lim _{n \in \omega} f_{n}$ se e somente se $\{n \in \omega: n \in$ $\left.f^{-1}[U]\right\} \in p$ para cada vizinhança aberta $U \subseteq X$ de $x$.

Como os elementos de um ultrafiltro não podem ser disjuntos, ao estarmos considerando espaços de Tychonoff uma seqüência tem no máximo um $p$-limite. Consequentemente, o $p$-limite de uma seqüência é único sempre que existir.

Nota 3.1.8. O fato de escolher $p$ como um ultrafiltro livre faz com que a definição não seja trivial. Com efeito, se $p$ for principal, digamos $p=\{S \subseteq \omega: k \in S\}$ para algum $k \in \omega$, observamos que qualquer seqüência $f: \omega \rightarrow X$ teria como $p$-limite ao ponto $f_{k}$.

Nenhum ultrafiltro livre $p \in \beta \omega$ contém conjuntos finitos; assim, se $x$ é $p$-limite $x$ de uma seqüência $f: \omega \rightarrow X$, para cada vizinhança aberta $U$ de $x$, temos que $f^{-1}[U]=\{n \in \omega$ : $f(n) \in U\} \in[\omega]^{\omega}$ isto é, $x$ é um ponto de acumulação da seqüência $f$. A afirmação recíproca também é verdadeira:

Proposição 3.1.9. Um ponto $x$ constitui um ponto de acumulação da seqüência $f: \omega \rightarrow X$ se e somente se existe $p \in \omega^{*}$ tal que $x=p-\lim _{n \in \omega} f_{n}$.

Demonstração. Vide [70].

Como é natural, as funções contínuas preservam os $p$-limites:

Proposição 3.1.10. Sejam $X$ e $Y$ espaços topológicos Hausdorff e $f: X \rightarrow Y$ uma função contínua. Se $\left\{x_{n}: n \in \omega\right\}$ é uma seqüência em $X$ tal que $p$ - $\lim _{n \in \omega} x_{n}$ existe, então $f\left(p-\lim _{n \in \omega} x_{n}\right)=$ $p-\lim _{n \in \omega} f\left(x_{n}\right)$.

Demonstração. Seja $z=p$ - $\lim _{n \in \omega} x_{n}$. Seja $W$ um aberto em $Y$ contendo o ponto $f(z)$, pela continuidade de $f, f^{-1}[W]$ é aberto em $X$ contendo o ponto $z$. Em conseqüência, $\{n \in \omega$ : $\left.x_{n} \in f^{-1}[W]\right\}=\left\{n \in \omega: f\left(x_{n}\right) \in W\right\} \in p$, ou seja $f(z)=p-\lim _{n \in \omega} f\left(x_{n}\right)$.

Usando a definição de $p$-limite, Bernstein introduziu a seguinte propriedade:

Definição 3.1.11. Um espaço $X$ é $p$-compacto se e somente se toda seqüência em $X$ possui -limite. 
A p-compacidade é uma propriedade topológica universal no sentido categórico, isto é, constitui uma propriedade hereditária sob subconjuntos fechados do espaço, a satisfazem todos os espaços compactos e é produtiva.

Teorema 3.1.12. Seja $p \in \omega^{*}$. Todo produto de espaços $p$-compactos é p-compacto.

Demonstração. Seja $\left\{X_{\alpha}: \alpha \in I\right\}$ uma família de espaços $p$-compactos e $X=\prod_{\alpha \in I} X_{\alpha}$. Suponha que $f: \omega \rightarrow X$ é uma seqüência em $X$. Para cada $\alpha \in I$ temos que

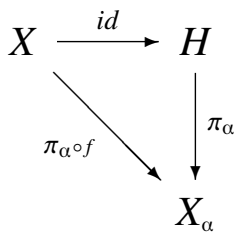

isto é, $\pi_{\alpha} \circ f: \omega \rightarrow X_{\alpha}$ é uma seqüência em $X_{\alpha}$, logo pela hipótese, existe $X_{\alpha} \ni x_{\alpha}=p$ - $\lim _{n \in \omega} \pi_{\alpha} \circ$ $f(n)$. Vamos mostrar que $x=\left(x_{\alpha}\right)_{\alpha \in I}=p-\lim _{n \in \omega} f(n)$. Seja $B=\prod_{\alpha \in I} U_{\alpha}$ um aberto básico em $X$ contendo o ponto $x$. Então, se $F=\left\{\alpha \in I: U_{\alpha} \neq X_{\alpha}\right\} \in[I]^{<\omega}$, para cada $j \in F$ temos que $\left\{n \in \omega: \pi_{j}\left(f_{n}\right) \in U_{j}\right\} \in p, \operatorname{logo}$

$$
\left\{n \in \omega: f_{n} \in B\right\} \supseteq \bigcap_{j \in F}\left\{n \in \omega: \pi_{j}\left(f_{n}\right) \in U_{j}\right\} \in p
$$

\section{$3.2 \quad p$-compacidade e ultrafiltros seletivos}

Como foi comentado no capítulo 2, nem a compacidade enumerável nem a pseudocompacidade são propriedades preservadas sob produtos de espaços topológicos gerais. Nesta direção, em [55], Saks estudou a produtividade da propriedade "enumeravelmente compacto para toda potência”, mostrando que essa propriedade é consistentemente não produtiva na classe dos espaços topológicos. Saks, então, perguntou se um exemplo desse fato pode ser obtido em ZFC sem assumir axiomas adicionais; Garcia-Ferreira mostrou que, de fato, a produtividade dessa propriedade é independente de ZFC. No entanto, dado que o exemplo obtido por Saks não é um espaço homogêneo, Garcia-Ferreira propôs a questão desta vez limitandose à classe dos grupos topológicos e assumindo adicionalmente MA. Uma maneira natural de estudar a propriedade em questão é feita por meio da ordem de Comfort, que usa o conceito de $p$-compacidade introduzido por Bernstein.

Definição 3.2.1. Dados $p, q \in \omega^{*}$ dizemos que $p \leq_{C} q\left(p \leq_{\mathrm{CG}} q\right)$ se todo espaço (grupo) $p$-compacto é $q$-compacto. Esta pré-ordem definida sobre $\omega^{*}$ chama-se de ordem de Comfort (ordem de grupo de Comfort) sobre $\omega^{*}$. 
A relação $\leq_{R K} \subseteq \leq_{C}$ é estabelecida no trabalho [35] mas as ordens $\leq_{R K}$ e $\leq_{C}$ não coincidem sobre $\omega / \approx_{\mathrm{RK}}$. De fato, Garcia Ferreira [34] mostrou em ZFC que para cada $p \in \omega^{*}$ existe $q \in \omega^{*}$ tal que $p \approx_{\mathrm{C}} q, p \leq_{\mathrm{RK}} q$ mas $p \not_{\mathrm{RK}} q$. No entanto, as ordens $\leq_{\mathrm{RK}} \mathrm{e} \leq_{\mathrm{C}}$ estão relacionadas de maneira especial, como mostram as seguintes afirmações devidas a Garcia Ferreira [34]:

- $p \leq_{\mathrm{C}} q$ se e somente se existe $r \in \omega^{*}$ tal que $r \approx_{\mathrm{C}} q$ e $p \leq_{\mathrm{RK}} r$.

- O espaço $\omega^{*}$ é um conjunto dirigido sob $\leq_{\mathrm{RK}}$ se e somente se é dirigido sob $\leq_{\mathrm{C}}$ (Blass e Shelah [13] mostraram que a primeira implicação é consistente com ZFC).

- Se $p$ é um P-ponto fraco, então as condições $p \leq_{\mathrm{RK}} q, p \leq_{\mathrm{C}} q$ e $p \leq_{\mathrm{CG}} q$ são equivalentes.

Em 1979, Saks mostrou que dois ultrafiltros seletivos incomparáveis são incompatíveis na ordem de Comfort. De outra parte, Garcia-Ferreira mostrou que no modelo de Shelah apresentado em [13], a ordem de Comfort e a ordem de grupo de Comfort são conjuntos dirigidos, isto é, todos os elementos dessas ordens são compatíveis. Daí, aparece a seguinte

Questão 3.1. MA implica que $\omega^{*}$ não é um conjunto dirigido considerando a ordem $\leq_{\mathrm{CG}}$ ?

Uma resposta afirmativa à questão anterior é equivalente a dizer que sob MA existem um grupo $p$-compacto $G$ e um grupo $q$-compacto $H$ tais que $G \times H$ não é $r$-compacto para todo $r \in \omega^{*}$. Neste ponto, cabe citar o célebre resultado de Ginsburg e Saks [36] acerca de potências de espaços enumeravelmente compactos:

Teorema 3.2.2. Seja X um espaço topológico. As seguintes afirmações são equivalentes:

a. X é p-compacto para algum $p \in \omega^{*}$;

b. $X^{\kappa}$ é enumeravelmente compacto para todo cardinal $\kappa$;

c. $X^{2^{c}}$ é enumeravelmente compacto.

De acordo com o teorema 3.2.2, uma resposta afirmativa à questão 3.1 é equivalente a mostrar sob MA a existência de dois grupos cujas potências são todas enumeravelmente compactas mas cujo produto não é enumeravelmente compacto.

Na verdade, a pergunta 3.1 escolhendo MA devido a que todos os exemplos que mostraram a não produtividade da compacidade enumerável na classe dos grupos topológicos exibidos até então usaram alguma forma desse axioma. 


\subsubsection{Ultraprodutos e $p$-compacidade}

Neste parágrafo lembraremos alguns fatos sobre ultraprodutos a partir de ultrafiltros seletivos, provando alguns resultados técnicos que serão usados para a obtermos grupos $p$ compactos.

Os ultraprodutos foram definidos por Łoś em 1955. A idéia é obter um grupo $p$-compacto $G$ de tamanho c por meio de um ultrafiltro seletivo $p \in \omega^{*}$, de modo que exista uma correspondência entre as seqüências de $G$ e os elementos do ultraproduto $\left([c]^{<\omega}\right)^{\omega} / p$. Vamos considerar dito ultraproduto como um espaço vetorial sobre o corpo $2=\{0,1\}$, cuja operação de "soma" estará determinada pela diferença simétrica $A \triangle B=A \backslash B \cup B \backslash A$.

Se $F \in[c]]^{<\omega}, \vec{F}$ representa a função em $\left([c]^{<\omega}\right)^{\omega} / p$ tal que para cada $n \in \omega, \vec{F}(n)=F$. Se $\alpha$ é um ordinal, simplificando a notação temos $\vec{\alpha}:=\{\overrightarrow{\alpha\}}$.

A seguir, definiremos o ultraproduto com que vamos trabalhar:

Definição 3.2.3. Seja $p \in \omega^{*}$. Dadas duas funções $f, g \in\left([\mathfrak{c}]^{<\omega}\right)^{\omega}$, dizemos que $f$ e $g$ são $p$-equivalentes se $\{n \in \omega: f(n)=g(n)\} \in p$. Nesse caso, escrevemos $f \approx_{p} g$.

$\approx_{p}$ define uma relação de equivalência sobre $\left([c]^{<\omega}\right)^{\omega}$. Com efeito

$\diamond \approx_{p}$ é reflexiva: Temos que $\{n \in \omega: f(n)=f(n)\}=\omega \in p$;

$\diamond \approx_{p}$ é simétrica: Se $f \approx_{p} g,\{n \in \omega: f(n)=g(n)\}=\{n \in \omega: g(n)=f(n)\} \in p, \log g \approx_{p} f$;

$\diamond \approx_{p}$ é transitiva: Se $f \approx_{p} g$ e $g \approx_{p} h$, então $\{n \in \omega: f(n)=g(n)\} \in p$ e $\{n \in \omega: g(n)=$ $h(n)\} \in p$. Como $p$ é um filtro, decorre que $p \ni\{n \in \omega: f(n)=h(n)\} \supset\{n \in \omega: f(n)=$ $g(n)\} \cap\{n \in \omega: g(n)=h(n)\}, \log f \approx_{p} h$.

Para cada $f \in\left([\mathrm{c}]^{<\omega}\right)^{\omega}$, representaremos por $[f]_{p}$ a classe de $p$-equivalência de $f$, isto é, o conjunto de todos os elementos $p$-equivalentes a $f$. O conjunto $\left([c]^{<\omega}\right)^{\omega} / p$ representa o conjunto de todas as classes de $p$-equivalência. Iremos nos referir a este conjunto como o ultraproduto de $[\mathrm{c}]^{<\omega}$ associado ao ultrafiltro $p \in \omega *$.

Definição 3.2.4. Seja $p \in \omega$. Para cada $f, g \in\left([c]^{<\omega}\right)^{\omega}$, definimos

$$
[f]_{p} \Delta[g]_{p} \stackrel{\text { def }}{=}[f \triangle g]_{p}
$$

onde, para cada $n \in \omega,(f \triangle g)(n) \stackrel{\text { def }}{=} f(n) \triangle g(n)$. 
Afirma-se que $\Delta$ constitui uma operação bem definida em $\left([c]^{<\omega}\right)^{\omega} / p$. Com efeito, sejam $f, g, h \in\left([\mathfrak{c}]^{<\omega}\right)^{\omega}$ e suponha-se que $[f]_{p}=[h]_{p}$. Deve-se mostrar que $[f \Delta g]_{p}=[h \triangle g]_{p}$. Se $k \in[f \triangle g]_{p}$, então existe $A \in p$ tal que $k(n)=f(n) \triangle g(n)$ para todo $n \in A$. Seja $B=\{n \in \omega$ : $f(n)=h(n)\} \in p$. Note que se $n \in A \cap B, k(n)=h(n) \triangle g(n), \operatorname{logo} A \cap B \subseteq\{n \in \omega: k(n)=$ $h(n) \triangle g(n)\}$. Dado que $p$ é um filtro, $\{n \in \omega: k(n)=h(n) \triangle g(n)\} \in p$, ou seja, $k \in[h \triangle g]_{p}$. Daí, $[f \triangle g]_{p}=[h \triangle g]_{p}$, pois duas classes de equivalência são disjuntas ou iguais. Do anterior decorre que $\Delta$ é uma operação bem definida sobre o ultraproduto $\left([c]^{<\omega}\right)^{\omega} / p$.

Como é natural, dado que a diferença simétrica de conjuntos é comutativa e associativa, temos que $\Delta$ é uma operação comutativa e associativa definida sobre $\left([c]^{<\omega}\right)^{\omega} / p$. Agora, seja $\vec{\varnothing} \in\left([\mathfrak{c}]^{<\omega}\right)^{\omega}$ a função constante definida como $\vec{\varnothing}(n)=\varnothing$ para todo $n \in \omega$. Então, para cada $f \in\left([\mathrm{c}]^{<\omega}\right)^{\omega}$ e $n \in \omega$ temos

$$
f \Delta \vec{\varnothing}(n)=f(n) \Delta \vec{\varnothing}(n)=f(n) \Delta \varnothing=f(n)
$$

Daí, $\{n \in \omega: f \Delta \vec{\varnothing}(n)=f(n)\}=\omega \in p$, de onde $[f \Delta \vec{\varnothing}]=[f]=[\vec{\varnothing} \Delta f]$, logo $[\vec{\varnothing}]_{p}$ é o elemento neutro da operação $\Delta$. Também se $f \in\left([\mathfrak{c}]^{<\omega}\right)^{\omega}$, para cada $n \in \omega$ temos que $f(n) \Delta f(n)=\varnothing, \operatorname{logo}[f]_{p} \Delta[f]_{p}=[f \triangle f]_{p}=[\vec{\varnothing}]_{p}$.

Do anterior, deduzimos que $\left(\left([\mathrm{c}]^{<\omega}\right)^{\omega} / p, \Delta\right)$ tem estrutura de grupo abeliano onde todos os elementos são de ordem 2 , constituindo deste modo um $\mathbb{Z}_{2}$-módulo. Como $\mathbb{Z}_{2}$ é de fato um corpo, $\left(\left([\mathfrak{c}]^{<\omega}\right)^{\omega} / p, \Delta\right)$ é um $\mathbb{Z}_{2}$-espaço vetorial, onde para cada $k \in \mathbb{Z}_{2}, k[f]_{p}=\underbrace{[f]_{p} \Delta \ldots \Delta[f]_{p}}_{k \text {-vezes módulo } 2}$.

Para este espaço vetorial, temos as seguintes propriedades:

Proposição 3.2.5. Se $\mathcal{A}=\left\{\left[g_{\alpha}\right]: \alpha \in I\right\}$ é uma família de classes de p-equivalência de $\left([\mathrm{c}]^{<\omega}\right)^{\omega} / p$, então

i. $\mathcal{A}$ é linearmente independente se e somente se para todo $F \in[I]^{<\omega}$ existe $A \in p$ tal que $\triangle\left\{g_{\alpha}(n): \alpha \in F\right\} \neq \varnothing$ para cada $n \in A ;$

ii. A gera o espaço vetorial $\left([\mathrm{c}]^{<\omega}\right)^{\omega} / p$ se e somente se para cada $g \in\left([\mathrm{c}]^{<\omega}\right)^{\omega}$ existem $A \in p e$ $F \in[I]^{<\omega}$ tais que para cada $n \in A, g(n)=\triangle\left(\left\{g_{\alpha}(n): \alpha \in F\right\}\right)$.

Demonstração. $\quad$ i. Dado que $\left([c]^{<\omega}\right)^{\omega} / p$ é booleano, uma combinação linear definida nele é, simplesmente, uma soma finita de elementos em $\left([\mathfrak{c}]^{<\omega}\right)^{\omega} / p$. Daí, $\left\{\left[g_{\alpha}\right]: \alpha \in I\right\}$ é linearmente independente se e somente se para qualquer $F \in[I]^{<\omega}, \Delta_{\alpha \in F}\left[g_{\alpha}\right] \neq[\vec{\varnothing}]$, ou seja, existe $A \in p$ tal que para cada $n \in A, \triangle_{\alpha \in F} g_{\alpha}(n) \neq \varnothing$. 
ii. A família $\left\{\left[g_{\alpha}\right]: \alpha \in I\right\}$ gera $\left([c]^{<\omega}\right)^{\omega}$ se e somente se para cada $g \in\left([c]^{<\omega}\right)^{\omega}$, existe $F \in[I]^{<\omega} \backslash\{\varnothing\}$ tal que $[g]=\triangle_{\alpha \in F}\left[g_{\alpha}\right]$, o que é satisfeito se e somente se $A:=\{n \in \omega:$ $\left.g(n)=\triangle_{\alpha \in F} g_{\alpha}(n)\right\} \in p$.

A seguinte proposição, faz a conexão entre a $p$-compacidade e os ultraprodutos sobre $p$ :

Proposição 3.2.6. Sejam $K$ um grupo topológico e $\phi:[c]^{<\omega} \rightarrow K$ um homomorfismo de grupo, $e \mathscr{A}=\left\{\left[g_{\alpha}\right]: \alpha \in \mathfrak{c}\right\} \subseteq\left([\mathfrak{c}]^{<\omega}\right)^{\omega} / p$, onde $p \in \omega^{*}$. Se $\mathscr{A}$ gera o espaço vetorial $\left([\mathfrak{c}]^{<\omega}\right)^{\omega} / p$ e para cada $\alpha<\mathfrak{c}$ a seqüência $\left\{\phi\left(g_{\alpha}\right)(n): n \in \omega\right\}$ tem p-limite em $\phi\left([\mathfrak{c}]^{<\omega}\right)$, então o espaço $\phi\left([\mathfrak{c}]^{<\omega}\right)$ é p-compacto.

Demonstração. Seja $\left\{y_{n}: n \in \omega\right\}$ uma seqüência no grupo $\phi\left([c]^{<\omega}\right)$. Para cada $n \in \omega$, escolhemos $g_{n} \in\left([\mathfrak{c}]^{<\omega}\right)^{\omega}$ de modo que $\phi\left(g_{n}\right)=y_{n}$; deste modo, estamos estabelecendo uma função $g \in\left([\mathfrak{c}]^{<\omega}\right)^{\omega}$ onde $g(n):=g_{n}$ para cada $n \in \omega$.

Pela proposição 3.2.5, dado que a família $\left\{\left[g_{\alpha}\right]: \alpha \in \mathfrak{c}\right\}$ gera $\left([\mathfrak{c}]^{<\omega}\right)^{\omega} / p$, existem $F \in[\mathfrak{c}]^{<\omega}$ e $A \in p$ tais que $g(n)=\triangle_{\xi \in F} g_{\xi}(n)$ para cada $n \in A$. Considere para cada $\xi \in F$, a seqüência em $\phi\left([\mathfrak{c}]^{<\omega}\right)$ dada por $\left\{\phi\left(g_{\xi}(n)\right): n \in \omega\right\}$. Por hipótese, existe $Z_{\xi} \in \phi\left([\mathfrak{c}]^{<\omega}\right)$ tal que $Z_{\xi}=$ $p-\lim _{n \in \omega} \phi\left(g_{\xi}(n)\right)$. Queremos provar que $\sum_{\xi \in F} Z_{\xi}$ é o $p$-limite da seqüência $\left\{y_{n}: n \in \omega\right\}$. Seja $U$ uma vizinhança de $\sum_{\xi \in F} Z_{\xi}$; dado que $K \supset \phi\left([\mathfrak{c}]^{<\omega}\right)$ é um grupo topológico, para cada $\xi \in F$ existe uma vizinhança $U_{\xi}$ de $z_{\xi}$ tal que $\sum_{\xi \in F} U_{\xi} \subseteq U$. Daí,

$$
\begin{aligned}
\left\{n \in A: y_{n} \in U\right\} & =\left\{n \in A: \phi\left(\bigwedge_{\xi \in F} g_{\xi}(n)\right) \in U\right\} \\
& =A \cap\left\{n \in \omega: \triangle_{\xi \in F} \phi\left(g_{\xi}(n)\right) \in U\right\} \\
& \supseteq A \cap \bigcap_{\xi \in F}\left\{n \in \omega: \phi\left(g_{\xi}(n)\right) \in U_{\xi}\right\}
\end{aligned}
$$

Para cada $\xi \in F, p-\lim _{n \in \omega} \phi\left(g_{\xi}(n)\right)=Z_{\xi}, \log 0\left\{n \in \omega: \phi\left(g_{\xi}(n)\right) \in U_{\xi}\right\} \in p$. Daí, $\bigcap_{\xi \in F}\{n \in \omega:$ $\left.\phi\left(g_{\xi}(n)\right) \in U_{\xi}\right\} \in p$. Visto que $A \in p$, obtemos o resultado.

Teorema 3.2.7. Sejam $K$ um grupo topológico, $\phi:[\mathfrak{c}]^{<\omega} \rightarrow K$ um homomorfismo de grupos $e$ $\left\{g_{\alpha}: \alpha<\mathfrak{c}\right\} \subseteq\left([\mathfrak{c}]^{<\omega}\right)^{\omega}$. Se $\left\{\left[g_{\alpha}\right]: \alpha<\mathfrak{c}\right\} \cup\{[\vec{\alpha}]: \alpha<\mathfrak{c}\}$ gera $\left([\mathfrak{c}]^{<\omega}\right)^{\omega} / p$ e para cada $\alpha<\mathfrak{c}$, $\left\{\phi\left(g_{\alpha}(n)\right): n \in \omega\right\}$ tem $p$-limite em $\phi\left([\mathfrak{c}]^{<\omega}\right)$, então $\phi\left([c]^{<\omega}\right)$ é p-compacto.

Demonstração. Notemos que para cada $\alpha<\mathfrak{c}$, a seqüência $\{\phi(\{\alpha\}): n \in \omega\}$ associada a $[\vec{\alpha}]$ é constante, de modo que possui esse ponto como $p$-limite. Pela proposição 3.2.6, decorre o resultado. 
Para estudar as construções deste capítulo, é preciso recorrer a alguns resultados técnicos envolvendo ultrafiltros seletivos.

Lema 3.2.8. Sejam $p$ um ultrafiltro seletivo $e\left\{a_{k}: k \in \omega\right\} \in p$ uma seqüência em $\omega$ estritamente crescente, tal que para cada $k \in \omega, k<a_{k}$. Então existe $I \subseteq \omega$ tal que

i. $\left\{a_{k}: k \in I\right\} \in p$;

ii. $\left\{\left[k, a_{k}\right]: k \in I\right\}$ é uma coleção de intervalos de $\omega$ que são dois a dois disjuntos.

Demonstração. Provaremos o teorema definindo uma partição $\left\{P_{0}, P_{1}\right\}$ de $[\omega]^{2}$ convenientemente para depois usar a caracterização do ultrafiltro $p$ dada nesses termos. Para cada $\{b, c\} \in[\omega]^{2}$, dizemos que $\{b, c\} \in P_{0}$ se e somente se existem $i, k \in \omega$ onde $i<k$ satisfazendo que $\{b, c\}=\left\{a_{i}, a_{k}\right\}$ e $a_{i}<k$.

Como $p$ é seletivo, pelo item v) do teorema 3.1.5, existe $B \in p$ tal que $[B]^{2} \subseteq P_{0}$ ou $[B]^{2} \subseteq P_{1}$.

Afirma-se que $[B]^{2} \nsubseteq P_{1}$. De fato, seja $\left\{t_{m}: n \in \omega\right\}$ uma seqüência de $\omega$, crescente de modo que $B=\left\{a_{t_{m}}: m \in \omega\right\}$. Suponha por absurdo que $[B]^{2} \subseteq P_{1}$; então, para cada $m>0$, $\left\{a_{t_{0}}, a_{t_{m}}\right\} \in P_{1}$. Como $P_{1}=P_{0}^{\mathrm{c}}$, segue que $a_{t_{0}} \geq t_{m}$ para cada $m>0$, absurdo pois $B$ é infinito. Portanto, $[B]^{2} \subseteq P_{0}$.

Seja $I \subseteq \omega$ tal que $B=\left\{a_{k}: k \in I\right\} \in p$. Notemos que se $i<k \in I,\left\{a_{i}, a_{k}\right\} \in[B]^{2} \subseteq P_{0}$ de modo que $i<a_{i}<k<a_{k}$ implicando assim que $\left[i, a_{i}\right] \cap\left[k, a_{k}\right]=\varnothing$.

Lema 3.2.9. Sejam $p_{0}$ e $p_{1}$ ultrafiltros seletivos incomparáveis. Se para cada $j=0,1,\left\{a_{k}^{j}: k \in\right.$ $\omega\} \in p_{j}$ representa uma seqüência crescente tal que $k<a_{k}^{j}$ para todo $k \in \omega$, então existem $I_{0}, I_{1} \subseteq \omega$ tais que

i. para cada $j \in 2,\left\{a_{k}^{j}: k \in I_{j}\right\} \in p_{j}$;

ii. $\left\{\left[k, a_{k}^{j}\right]: k \in I_{j}, j \in 2\right\}$ é uma família de intervalos de $\omega$ dois a dois disjuntos.

Demonstração. Aplicando o lema 3.2.8 aos ultrafiltros $p_{0}$ e $p_{1}$ obtemos $L_{0}, L_{1} \subseteq \omega$ satisfazendo para cada $j \in 2$ :

1. $B_{j} \stackrel{\text { def }}{=}\left\{a_{k}^{j}: k \in L_{j}\right\} \in p_{j} \mathrm{e}$

2. a coleção $\left\{\left[k, a_{k}^{j}\right]: k \in L_{j}\right\}$ está formada por intervalos de $\omega$ que são dois a dois disjuntos. Como $p_{0}$ e $p_{1}$ são ultrafiltros distintos, sem perda de generalidade podemos supor adicionalmente 
3. $B_{0} \cap B_{1}=\varnothing$.

A idéia é achar refinamentos $I_{0}$ e $I_{1}$ das coleções $L_{0}$ e $L_{1}$ respectivamente, de forma tal que as condições i) e ii) sejam satisfeitas por eles. Observemos que pela propriedade B), para obter ii) é suficiente que $I_{0}$ e $I_{1}$ satisfaçam

4. $\left[i, a_{i}^{0}\right] \cap\left[k, a_{k}^{1}\right]=\varnothing$ para cada $(i, k) \in I_{0} \times I_{1}$.

Suponhamos que $B_{0}=\left\{a_{t_{m}}^{0}: m \in \omega\right\}$ onde $L_{0}=\left\{t_{m}: m \in \omega\right\}$ está ordenado de maneira crescente. Consideremos a partição de $\omega$ dada por $\left\{\left[a_{t_{2 m}}^{0}, a_{t_{2 m+1}}^{0}\right]: m \in \omega\right\} \cup\left\{\left[0, a_{t_{0}}^{0}\right] \backslash B_{0}\right\}$. Como $p_{1}$ é seletivo, temos que existe $C_{1} \in p_{1}$ tal que para cada $m \in \omega,\left|\left[a_{t_{2 m}}^{0}, a_{t_{2 m+1}}^{0}\right] \cap C_{1}\right| \leq 1$. Como $C_{1} \cap B_{1} \in p_{1}$ podemos assumir sem perda de generalidade que $C_{1} \subseteq B_{1}$.

Seja $T=\bigcup\left\{\left[i, a_{i}^{0}\right]: a_{i}^{0} \in B_{0}\right.$, e $\left.\left[i, a_{i}^{0}\right] \cap B_{1}=\varnothing\right\}$. Definimos $f_{0}: \omega \rightarrow \omega:$

- $f_{0} \uparrow_{\omega, T}$ de modo arbitrário;

- se $n \in T$, existe um único $t \in \omega$ tal que $n \in\left[t, a_{t}^{0}\right]$ e um único $m \in \omega$ tal que $\{m\}=$ $\left[t, a_{t}^{0}\right] \cap C_{1}$. Tomemos neste caso, $f_{0}(n)=m$.

Como $p_{0}$ e $p_{1}$ são incomparáveis, necessariamente $f_{0}\left(p_{0}\right) \neq p_{1}$. Daí, existe $D_{1} \in P_{1}$ tal que $f_{0}^{-1}\left[D_{1}\right] \notin P_{0}$.

Seguindo um argumento similar ao feito antes, podemos supor também que $D_{1} \subseteq C_{1}$.

Seja $C_{0} \stackrel{\text { def }}{=} B_{0} \cap f_{0}^{-1}\left[D_{1}\right] \in p_{0}$. Afirmamos que se $a_{i}^{0} \in C_{0}$ então $\left[i, a_{i}^{0}\right] \cap D_{1}=\varnothing$; com efeito, se $a_{t}^{1} \in\left[i, a_{i}^{0}\right] \cap D_{1}$, então $\left\{a_{t}^{1}\right\}=\left[i, a_{i}^{0}\right] \cap C_{1}$, logo, de acordo com a definição de $f$, $f_{0}\left(a_{i}^{0}\right)=a_{t}^{1} \in D_{1}$, ou seja $a_{i}^{0} \in f_{0}^{-1}\left[D_{1}\right]$ o que contradiz a definição de $C_{0}$.

Suponhamos que $D_{1}=\left\{a_{i}^{1}: i \in I\right\}$ onde $I=\left\{r_{m}\right\}$ é uma seqüência crescente de elementos de $\omega$ sendo também assim a enumeração de $D_{1}$ feita por $I$. Considere a partição de $\omega$ dada pela família $\left\{\left[a_{r_{2 n}}^{1}, a_{r_{2 n+1}}^{1}\right]: n \in \omega\right\} \cup\left\{\left[0, a_{r_{0}}^{1}\right] \backslash D_{1}\right\}$. Como $p_{0}$ é seletivo, existe $D_{0} \in p$ tal que $D_{0} \subseteq C_{0}$ e para cada $n \in \omega,\left|\left[a_{r 2 n}^{1}, a_{r_{2 n+1}}^{1}\right] \cap D_{0}\right| \leq 1$.

Seja $S=\bigcup\left\{\left[i, a_{i}^{1}\right]: a_{i}^{1} \in C_{1},\left[i, a_{i}^{1}\right] \cap C_{0} \neq \varnothing\right\} \subseteq \omega$. Para cada $n \in S$, existe um único $t \in \omega$ tal que $n \in\left[t, a_{t}^{1}\right]$ e um único $m \in \omega$ tal que $\{m\}=\left[t, a_{t}^{1}\right] \cap D_{0}$. De acordo com isto, seja $f_{1}(n)=m$. Escolhendo de maneira arbitrária os valores $f_{1}(l)$ para cada $l \in \omega \backslash S$, obtemos uma função $f_{1}: \omega \rightarrow \omega$.

Dado que $p_{0}$ e $p_{1}$ são $\leq_{\mathrm{RK}}$-incomparáveis, $f_{1}\left(p_{1}\right) \neq p_{0}$; assim, existe $E_{0} \in p_{0}$ tal que $E_{0} \subseteq D_{0}$ e $f_{1}^{-1} \notin p_{1}$. Seja $E_{1}=D_{1} \backslash f_{1}^{-1}\left[E_{0}\right] \in p_{1}$. Mediante argumentos similares aos anteriores, temos que se $a_{i}^{1} \in E_{1}$, então $\left[i, a_{i}^{1}\right] \cap E_{0}=\varnothing$. Observemos que $E_{0} \subseteq D_{0} \subseteq C_{0}$; portanto, se $a_{i}^{0} \in E_{0}$ então $\left[i, a_{i}^{0}\right] \cap E_{1}=\varnothing$. 
Para cada $j \in\{0,1\}$, seja $I_{j} \subseteq \omega$ tal que $E_{j}=\left\{a_{k}^{j}: k \in I_{j}\right\}$. Notemos que para cada $j \in\{0,1\}, E_{j} \in p_{j}$, logo os conjuntos de índices $I_{0}$ e $I_{1}$ satisfazem a condição i) do lema.

Agora, suponhamos, a procura de uma contradição, que existem $n \in I_{0}$ e $m \in I_{1}$ tais que $\left[n, a_{n}^{0}\right] \cap\left[m, a_{m}^{1}\right] \neq \varnothing$. Pela maneira em que foi feita a construção das coleções envolvidas, temos que dados $r \in I_{0}, s \in I_{1},\left[r, a_{r}^{0}\right] \cap\left[s, a_{s}^{1}\right] \subseteq\left(\omega \backslash I_{0}\right) \cap\left(\omega \backslash I_{1}\right)$, o que implica $r \neq s$, ou seja $I_{0} \cap I_{1}=\varnothing$. De acordo com isto, pela hipótese temos que necessariamente $a_{n}^{0} \in\left[m, a_{m}^{1}\right]$ ou $a_{m}^{1} \in\left[n a_{n}^{0}\right]$ o que contradiz a escolha dos conjuntos $E_{0}$ e $E_{1}$. Isto conclui a prova.

Lema 3.2.10. Seja $\left\{p_{j}: j \in \omega\right\}$ uma família de ultrafiltros seletivos $\leq_{\mathrm{RK}}$-incomparáveis. Se para cada $j \in \omega,\left\{a_{k}^{j}: k \in \omega\right\} \in p_{j}$ representa uma seqüência crescente tal que para cada $k \in \omega$, $k<a_{k}^{j}$, então existe uma família $\left\{I_{j}: j \in \omega\right\} \subseteq[\omega]^{\omega}$ satisfazendo as seguintes condições:

i. para cada $j \in \omega,\left\{a_{k}^{j}: k \in I_{j}\right\} \in p_{j}$;

ii. $\left\{\left[k, a_{k}^{j}\right]: j \in \omega, k \in I_{j}\right\}$ é uma família de intervalos em $\omega$ dois a dois disjuntos.

Demonstração. Por indução, para cada $j \in \omega$, vamos construir uma família de subconjuntos de $\omega,\left\{I_{j, t}: t \in \omega\right\}$ tal que:

A-1. para cada $t \in \omega, I_{j, t+1} \subseteq I_{j, t}$;

A-2. a família $\left\{\left[k, a_{k}^{j}\right]: j \leq t\right.$ e $\left.k \in I_{j, t}\right\}$ está formada por intervalos de $\omega$ dois a dois disjuntos;

A-3. $\left\{a_{k}^{j}: k \in I_{j, t}\right\} \in p_{j}$.

Pelo lema 3.2.8, existe $I_{0,0} \subseteq \omega$ tal que $\left\{a_{k}^{0}: k \in I_{0,0}\right\} \in p_{0}$ e $\left\{\left[k, a_{k}^{0}\right]: k \in I_{0,0}\right\}$ é uma família de intervalos de $\omega$ dois a dois disjuntos. é claro que as condições a)-c) são satisfeita para $j, t \leq 0$.

Suponhamos agora que para cada $j, t \leq s$, temos definido os conjuntos $I_{j, t}$ satisfazendo as propriedades A-1)-A-3). Seja $I_{s+1,0}=\omega$. Observando que $I_{0, s}$ já esta definido e que $p_{s+1}$ e $p_{0}$ são $\leq_{\mathrm{RK}}$-incomparáveis, ao aplicar o lema 3.2.9 para os ultrafiltros e seqüências correspondentes, obtemos $I_{s+1,1} \subseteq I_{s+1,0}$ e $I_{0, s+1} \subseteq I_{0, s}$ tais que a família de intervalos em $\omega,\left\{\left[k, a_{k}^{0}\right]: k \in I_{0, s+1}\right\} \cup$ $\left\{\left[k, a_{k}^{s+1}\right]: k \in I_{s+1,1}\right\}$ é dois a dois disjunta e $\left\{a_{k}^{0}: k \in I_{0, s+1}\right\} \in p_{0},\left\{a_{k}^{s+1}: k \in I_{s+1,1}\right\} \in p_{s+1}$.

Aplicando novamente o lema 3.2.9 mas desta vez as seqüências indexadas pelos conjuntos $I_{s+1,1}$ e $I_{1, s} \subseteq \omega$ que constituem elementos dos ultrafiltros $p_{s+1}$ e $p_{1}$ respetivamente, obtemos $I_{s+1,2} \subseteq I_{s+1,1}$ e $I_{1, s+1} \subseteq I_{1, s}$ tais que a coleção $\left\{\left[k, a_{k}^{1}\right]: k \in I_{1, s+1}\right\} \cup\left\{\left[k, a_{k}^{s+1}\right]: k \in I_{s+1,2}\right\}$ está formada por intervalos em $\omega$ dois a dois disjuntos e $\left\{a_{k}^{1}: k \in I_{1, s+1}\right\} \in p_{1},\left\{a_{k}^{s+1}: k \in I_{s+1,2}\right\} \in p_{s+1}$. Depois de aplicar o lema 3.2.9 $s$ vezes, obtemos para cada $t \leq s$ coleções de índices $I_{t, s+1}, I_{s+1, t+1}$ satisfazendo: 
B-1. para cada $t \leq s, I_{t, s+1} \subseteq I_{s+1, t+1}$ e $I_{s+1, t+1} \subseteq I_{s+1, t}$;

B-2. a coleção $\left\{\left[k, a_{k}^{t}\right]: k \in I_{t, s+1}\right\} \cup\left\{\left[k, a_{k}^{s+1}\right]: k \in I_{s+1, t+1}\right\}$ está formada por intervalos de $\omega$ dois a dois disjuntos;

B-3. para cada $t \leq s,\left\{a_{k}^{t}: k \in I_{t, s+1}\right\} \in p_{t}$ e $\left\{a_{k}^{s+1}: k \in I_{s+1, t+1}\right\} \in p_{s+1}$.

De acordo com a construção, temos que as condições A-1) e A-3) são satisfeitas para cada par $j, t \leq s+1$; pela hipótese de indução só falta mostrar que a condição A-2) é satisfeita tomando $t=s+1$. Suponhamos por absurdo que $\left\{\left[k, a_{k}^{j}\right]: j \leq s+1\right.$ e $\left.k \in I_{j, s+1}\right\}$ não satisfaz A-2). Então, existem $r, \check{r} \leq s+1, n \in I_{r, s+1}, m \in I_{\check{r}, s+1}$, tais que

$$
\left[n, a_{n}^{r}\right] \cap\left[m, a_{m}^{\check{r}}\right] \neq \varnothing
$$

Pela propriedade B-2), temos que $\check{r} \neq s+1$ ou $r+1 \neq s+1$, logo $\breve{r} \leq s$ ou $r<s$. Se ocorre $\check{r} \leq s$, pela hipótese indutiva a coleção $\left\{\left[k, a_{k}^{j}\right]: j \leq s, k \in I_{j, s}\right\}$ é dois a dois disjunta. Note que $I_{\check{r}, s+1} \subseteq I_{\check{r}, s}$ e $I_{r, s+1} \subseteq I_{r, s} \operatorname{logo}(*)$ implica que $r=s+1$; mas, por B-2), os membros de $\left\{\left[k, a_{k}^{\check{r}}\right]: k \in I_{\check{r}, s+1}\right\} \cup\left\{\left[k, a_{k}^{s+1}\right]: k \in I_{s+1, s+1}\right\}$ são dois a dois disjuntos, o que contradiz (*).

Se $r<s$, pela hipótese de indução deve-se satisfazer que $\check{r}=s+1$; de novo, pela propriedade B-2), os membros de $\left\{\left[k, a_{k}^{r}\right]: k \in I_{r, s+1}\right\} \cup\left\{\left[k, a_{k}^{s+1}\right]: k \in I_{s+1, s+1}\right\}$ são intervalos dois a dois disjuntos, contradizendo (*). Em conseqüência, temos que as condições A-1)-A-3) são satisfeitas para todos os pares $j, t \leq s+1$.

Por indução, para cada $j, t \in \omega$ os conjuntos de índices $I_{j, t} \subseteq \omega$ podem ser construídos satisfazendo A-1)-A-3).

Para cada $j \in \omega$, consideremos a coleção $\left\{\left\{a_{k}^{j}: k \in I_{j, t}\right\}: t \in \omega\right\} \subseteq p_{j}$. Como $p_{j}$ é um ultrafiltro seletivo, em particular constitui um $P$-ponto, de modo que existe $\widehat{U}_{j} \in p_{j}$ tal que para cada $t \in \omega$ o conjunto $\widehat{U}_{j} \backslash\left\{a_{k}^{j}: k \in I_{j, t}\right\}$ é finito. Note-se que se definirmos $U_{j}=\widehat{U}_{j} \cap\left\{a_{k}^{j}: k \in I_{j, j}\right\}, U_{j} \in p_{j}$ e para cada $t \in \omega, U_{j} \backslash\left\{a_{k}^{j}: k \in I_{j, t}\right\}$ é finito. Em conseqüência, se $U_{j}=\left\{a_{k}^{j}: k \in K_{j}\right\}$, então $K_{j} \subseteq I_{j, j}$ e para cada $t \in \omega, K_{j} \backslash I_{j, t}$ é finito. Em conseqüência, $\bigcup_{l \leq j}\left(K_{l} \backslash I_{l, j}\right)$ é finito logo é possível achar $M_{j} \in \omega$ de modo que $\bigcup_{l \leq j}\left(K_{l} \backslash I_{l, j}\right) \subseteq M_{j}$, satisfazendo-se então, para cada $l \leq j, K_{l} \backslash I_{l, j} \subseteq M_{j}$, ou seja, $K_{l} \subseteq I_{l, j} \cup M_{j}$.

Seja $I_{j} \stackrel{\text { def }}{=} K_{j} \backslash \max \left\{a_{k}^{l}+1: l<j, k<M_{j}\right\}$. Dado que $K_{j} \backslash I_{j}$ é finito, $\left\{a_{k}^{j}: k \in K_{j} \backslash I_{j}\right\} \notin p_{j}$. Como $p_{j} \in \omega^{*}$, então $\omega \backslash\left\{a_{k}^{j}: k \in K_{j} \backslash I_{j}\right\} \in p_{j}$, isto é

$$
\begin{aligned}
\left\{a_{k}^{j}: k \in I_{j}\right\} & =\left\{a_{k}^{j}: k \in K_{j}\right\} \cap\left\{a_{k}^{j}: k \in K_{j}\right\}^{\mathrm{c}} \\
& =U_{j} \cap\left\{a_{k}^{j}: k \in K_{j}\right\}^{\mathrm{c}} \in p_{j}
\end{aligned}
$$


isto é, $I_{j}$ satisfaz i).

A seguir, vamos mostrar que a família $\left\{I_{j}: j \in \omega\right\}$ satisfaz a condição ii). Para cada $i \in 2$, sejam $j, \breve{\jmath}, k, \breve{k} \in \omega$ tais que $k \in I_{j}, \breve{k} \in I_{\breve{j}}$. Se $j=\breve{\jmath}$, dado que $K_{j} \subseteq I_{j, j}$, temos que $k, \breve{k} \in I_{j} \subseteq I_{j, j}$. Pela propriedade B-2), temos que $\left[k, a_{k}^{j}\right] \cap\left[\breve{k}, a_{\breve{k}}^{j}\right]=\varnothing$ sempre que $k \neq \breve{k}$, $\operatorname{logo}$ neste caso a condição ii) é satisfeita. Suponhamos agora que $j<\check{j}$. De acordo com a

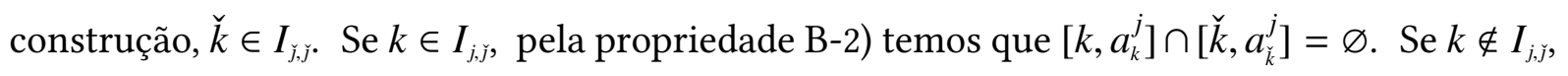
dado que $I_{j} \subseteq K_{j} \subseteq I_{j, j} \cup M_{\tilde{j}}$, temos $k \in M_{j}$; de acordo com a construção, para cada $n \in I_{\tilde{j}}$, $n>a_{k}^{j}$, em particular, $k>a_{k}^{j}$. Daí, $\left[k, a_{k}^{j}\right] \cap\left[\check{k}, a_{\breve{k}}^{\breve{j}}\right]=\varnothing$, obtendo-se o resultado.

\subsection{2 $\omega^{*}$ não é um conjunto dirigido na ordem $\leq_{\mathrm{CG}}$}

Nesta seção, vamos detalhar a solução proposta por A. Tomita e S. Watson em [69] à questão 3.1. Primeiro, é preciso construir certos homomorfismos que vão permitir "mergulhar"o conjunto $[\mathrm{c}]^{<\omega}$ em $2^{\mathrm{c}}$.

Lema 3.2.11. Sejam $p_{0}$ e $p_{1} \in \omega^{*}$ ultrafiltros seletivos $\leq_{\mathrm{RK}}$-incomparáveis. Sejam $F \in[\mathrm{c}]^{<\omega}$, $E \in[c]^{\omega}$ tais que $F \subseteq E$ e consideremos também a coleção de funções $\left\{g_{\xi}: \xi \in E\right\} \subseteq\left([E]^{<\omega}\right)^{\omega}$. Se $S_{0}, S_{1} \subseteq E$ são tais que para cada $j \in\{0,1\}$ o conjunto $\left\{\left[g_{\xi}\right]_{p_{j}}: \xi \in S_{j}\right\} \cup\left\{[\vec{\mu}]_{p_{j}}: \mu \in E\right\}$ é linearmente independente no ultraproduto $\left([\mathrm{c}]^{<\omega}\right)^{\omega} / p_{j}$, então existem uma função $r \in 2^{\omega}$, uma seqüência crescente $\left\{b_{i}: i \in \omega\right\} \subseteq \omega$ e uma família $\left\{E_{i}: i \in \omega\right\} \subseteq[E]^{<\omega}$ satisfazendo
a. $F \subseteq E_{0}$;
b. $E=\bigcup_{i \in \omega} E_{i}$;
c. para cada $i \in \omega, E_{i} \cup \bigcup_{\xi \in E_{i}} g_{\xi}\left(b_{i}\right) \subseteq E_{i+1}$;
d. para cada $i \in \omega$, o conjunto $\left\{g_{\xi}\left(b_{i}\right): \xi \in E_{i} \cap S_{r(i)}\right\} \cup\left\{\{\mu\}: \mu \in E_{i}\right\}$ é linearmente independente;
e. para cada $j \in\{0,1\},\left\{b_{k}: k \in r^{-1}[j]\right\} \in p_{j}$.

Demonstração. Antes de nada, iremos definir uma coleção $\left\{F_{n}: n \in \omega\right\} \subseteq[E]^{<\omega}$ a partir da qual é construída a família $\left\{E_{i}: i \in \omega\right\}$. Indutivamente, temos:

1. $F_{0} \stackrel{\text { def }}{=} F$;

2. para cada $n \geq 1$, supondo que $F_{n} \subseteq E$ já foi construído, tomamos $F_{n+1} \in[E]^{<\omega}$ satisfazendo $F_{n} \cup \bigcup\left\{g_{\beta}(m): m \leq n, \beta \in F_{n}\right\} \subseteq F_{n+1}$;

3. $E=\bigcup_{n \in \omega} F_{n}$. 
Para cada $j \in\{0,1\}$ e $n \in \omega$, seja

$$
A_{n}^{j} \stackrel{\text { def }}{=}\left\{k \in \omega:\left\{g_{\xi}(k): \xi \in F_{n} \cap S_{j}\right\} \cup\left\{\{\mu\}: \mu \in F_{n}\right\} \text { é linearmente independente }\right\}
$$

Por hipótese, para cada $j \in\{0,1\},\left\{\left[g_{\xi}\right]_{p_{j}}: \xi \in S_{j}\right\} \cup\left\{[\vec{\mu}]_{p_{j}}: \mu \in E\right\}$ é um conjunto linearmente independente logo

$$
W_{j}=\left\{k \in \omega:\left\{g_{\xi}(k): \xi \in S_{j}\right\} \cup\{\mu: \mu \in E\} \text { é linearmente independente }\right\} \in p_{j}
$$

Notemos que para cada $n \in \omega$, satisfaz-se $W_{j} \subseteq A_{n}^{j}$; daí, para cada $n \in \omega, A_{n}^{j} \in p_{j}$. Se considerarmos a coleção $\left\{A_{n}^{j}: n \in \omega\right\} \subseteq p_{j}$, dado que $p_{j}$ é seletivo e assim constitui um $P$-ponto em $\omega^{*}$, conclui-se que existe $A_{j} \in p_{j}$ tal que para cada $n \in \omega, A_{j} \subseteq^{*} A_{n}^{j}$. Daí, pode ser construída uma função crescente $h_{j}: \omega \rightarrow \omega$ satisfazendo para cada $n \in \omega A_{j} \backslash A_{n}^{j} \subseteq h_{j}(n)$.

Consideremos a partição de $\omega$ dada por $\left\{h_{j}(1)\right\} \cup\left\{\left[h_{j}(n)+1, h_{j}(n+1)\right]: n \in \omega\right\}$. Como $p_{j}$ é seletivo, tem-se que existe $B_{j} \in p_{j}$ tal que $B_{j} \cap h_{j}(1)=\varnothing, B_{j} \subseteq A_{j}$ e para cada $n \in \omega$, $\left|\left[h_{j}(n)+1, h_{j}(n+1)\right] \cap B_{j}\right| \leq 1$.

Suponhamos que $B_{j}=\left\{a_{n}^{j}: n \in \omega\right\}$ onde a enumeração é escolhida de maneira crescente. De acordo com a definição de $B_{j}$, temos $a_{k}^{j}>h_{j}(n)$ para cada $n, k \in \omega$ e particularmente temos que $a_{n}^{j}>h_{j}(n)$. Dado que $h_{j}$ é crescente, $n \leq h_{j}(n)<a_{n}^{j}$ e $a_{n}^{j} \in A_{n}^{j}$ para cada $n \in \omega$. Em virtude do anterior, temos que as seqüências $B_{0}=\left\{a_{n}^{0}: n \in \omega\right\}$ e $B_{1}=\left\{a_{n}^{1}: n \in \omega\right\}$ satisfazem as condições do lema 3.2.9. Aplicando este lema, obtemos coleções $I_{0}, I_{1} \subseteq \omega$ satisfazendo

- para cada $j \in\{0,1\},\left\{a_{k}^{j}: k \in I_{j}\right\} \in p_{j}$;

- o conjunto $\left\{\left[k, a_{k}^{j}\right]: j \in 2, k \in I_{j}\right\}$ está formado por intervalos de $\omega$ dois a dois disjuntos.

A última condição das enunciadas acima, implica que $I_{0} \cap I_{1}=\varnothing$, logo podemos tomar uma enumeração crescente de $I_{0} \cup I_{1} \stackrel{\text { def }}{=}\left\{i_{m}: m \in \omega\right\}$ e definir uma função $r: \omega \rightarrow 2$ de modo que $r(m)=j$ se e somente se $i_{m} \in I_{j}$. Baseados nisto, podemos definir para cada $m \in \omega$

$$
b_{m} \stackrel{\text { def }}{=} a_{i_{m}}^{r(m)}, \quad E_{m} \stackrel{\text { def }}{=} F_{i_{m}}
$$

Verifiquemos agora que as coleções $\left\{b_{i}: i \in \omega\right\},\left\{E_{i}: i \in \omega\right\}$ e a função $r \in 2^{\omega}$ que temos definido, satisfazem, de fato, a tese do teorema.

Inicialmente, tomamos $F_{0}=F$. Da construção, $E_{0}=F_{i_{0}} \supseteq F_{0}$, de modo que $\left\{E_{n}: n \in \omega\right\}$ satisfaz a condição a). 
Como $E=\bigcup_{n \in \omega} F_{n},\left\{F_{n}: n \in \omega\right\}$ é uma coleção $\subseteq$-crescente e $I_{0} \cup I_{1}$ é infinito, temos também que $E=\bigcup_{m \in \omega} F_{i_{m}}=\bigcup_{m \in \omega} E_{m}$, de modo que b) é satisfeita.

Notemos que $\left\{b_{m}: m \in \omega\right\}=\left\{a_{i_{m}}^{r(m)}: m \in \omega\right\}$; dai, se $m<n$ sabemos que $i_{m}<i_{n}$; dado que $\left[i_{m}, a_{i_{m}}^{r(m)}\right] \cap\left[i_{n}, a_{i_{n}}^{r(n)}\right]=\varnothing$, necessariamente $a_{i_{m}}^{r(m)}<a_{i_{n}}^{r(n)}$, ou seja $b_{m}<b_{n}$ logo a seqüência formada por estes termos é crescente. De acordo com a definição de $r$, para cada $j \in 2$,

$$
\left\{b_{k}: k \in r^{-1}[j]\right\}=\left\{a_{i_{k}}^{r(k)}: k \in r^{-1}[j]\right\}=\left\{a_{i_{k}}^{j}: i_{k} \in I_{j}\right\} \in p_{j}
$$

obtendo-se a condição e).

Para cada $m \in \omega, b_{m}=a_{i_{m}}^{r(m)} \in A_{i_{m}}^{r(m)}$. Portanto, para cada $n \in \omega$ o conjunto

$$
\left\{g_{\xi}\left(b_{m}\right): \xi \in F_{i_{m}} \cap S_{r(m)}\right\} \cup\left\{\{\mu\}: \mu \in F_{i_{m}}\right\}=\left\{g_{\xi}\left(b_{m}\right): \xi \in E_{m} \cap S_{r(m)}\right\} \cup\left\{\{\mu\}: \mu \in E_{m}\right\}
$$

é linearmente independente, isto é, obtém-se a condição d).

Da construção, temos que para cada $n \in \omega, a_{i_{m}}^{r(m)} \notin\left[i_{m+1}, a_{i_{m+1}}^{r(m)}\right], \operatorname{logo} b_{m}=a_{i_{m}}^{r(m)} \leq i_{m+1}-1$; note-se também que $E_{m}=F_{i_{m}} \subseteq F_{i_{m+1^{-1}}}$. Em conseqüência, para cada $m \in \omega$,

$$
E_{m} \cup\left\{g_{\xi}\left(b_{m}\right): \xi \in E_{m}\right\} \subseteq F_{i_{m+1}-1} \cup \bigcup\left\{g_{\xi}(k): k \leq i_{m+1}-1, \xi \in F_{i_{m+1^{-1}}}\right\} \subseteq F_{i_{m+1}}=E_{m+1}
$$

ou seja, a condição c) está também satisfeita, completando-se a prova.

Lema 3.2.12. Sejam $p_{0}$ e $p_{1}$ ultrafiltros seletivos $\leq_{\mathrm{RK}}$-incomparáveis. Se $F^{0}, F^{1} \subseteq[\mathfrak{c}]^{<\omega}$ são conjuntos distintos, $\left\{g_{\xi}: \xi<\mathfrak{c}\right\} \subseteq\left([\mathfrak{c}]^{<\omega}\right)^{\omega}$ é uma família de funções tais que $\bigcup_{n \in \omega} g_{\xi}(n) \subseteq \xi$, e para cada $i \in 2$ a coleção $\left\{\left[g_{\xi}\right]_{p_{i}}: \xi \in S_{i}\right\} \cup\left\{[\vec{\alpha}]_{p_{i}}: \alpha<\mathfrak{c}\right\}$ é linearmente independente no ultraproduto $\left([\mathrm{c}]^{<\omega}\right)^{\omega} / p_{i}$, então existem homomorfismos $\left.\phi^{0}, \phi^{1} \in \operatorname{hom}([c]]^{<\omega}, 2\right)$ satisfazendo:

i. Para cada $j \in 2$,

a. $\phi^{j}\left(F^{j}\right)=1$ sempre que $F^{j} \neq \varnothing$, e,

b. para cada $\xi \in S_{j},\left\{n \in \omega: \phi^{j}\left(g_{\xi}(n)\right)=\phi^{j}(\{\xi\})\right\} \in p_{j}$;

ii. o conjunto $\left\{n \in \omega:\left(\phi^{0}(\{n\}), \phi^{1}(\{n\})\right)=\left(\phi^{0}\left(F^{0}\right), \phi^{1}\left(F^{1}\right)\right)\right\}$ é finito.

Demonstração. Seja $F \stackrel{\text { def }}{=} F^{0} \cup F^{1}$; podemos achar um subconjunto $E \in[\mathfrak{c}]^{\omega}$ de modo que $F \cup \omega \subseteq E$ e para cada $\xi \in E$ e $n \in \omega, g_{\xi}(n) \subseteq E$. Se consideramos os conjuntos $F, E,\left\{g_{\xi}\right.$ : $\xi \in E\}$ e $\left\{S_{j} \cap E: j \in 2\right\}$, mediante o lema precedente obtemos coleções $\left\{b_{i}: i \in \omega\right\} \subseteq \omega$, $\left\{E_{i}: i \in \omega\right\} \subseteq \wp(E)$ e uma função $r \in 2^{\omega}$ satisfazendo

1. $F \subseteq E_{0}$; 
2. $E=\bigcup_{i \in \omega} E_{i}$;

3. para cada $i \in \omega, E_{i} \cup \bigcup_{\xi \in E_{i}} g_{\xi}\left(b_{i}\right) \subseteq E_{i+1}$;

4. para cada $i \in \omega,\left\{g_{\xi}\left(b_{i}\right): \xi \in E_{i} \cap S_{r(i)}\right\} \cup\left\{\{\mu\}: \mu \in E_{i}\right\}$ é linearmente independente;

5. para cada $j \in 2,\left\{b_{i}: i \in r^{-1}[j]\right\} \in p_{j}$.

Para cada $j \in 2$, em primeiro lugar vamos definir $\phi^{j}$ sobre $[E]^{<\omega}$ por indução em $\omega$. Para cada $j \in 2$, tomemos $\phi^{j}\left(F^{j}\right)=1$ se $F^{j} \neq \varnothing$ e estendamos $\phi^{j}$ a um homomorfismo definido sobre $\left[E_{0}\right]^{<\omega}$; em concordância com o anterior, note-se que o item i) já estaria satisfeito pelos homomorfismos $\phi^{0}$ e $\phi^{1}$. O passo seguinte da indução é agora definir para cada $n \in \omega$, o homomorfismo $\phi^{j}$ convenientemente sobre o subgrupo $\left[E_{n}\right]^{<\omega}$ de modo que as seguintes duas condições sejam satisfeitas

A-i) para cada $m \leq n$ e $\xi \in S_{r(m)} \cap E_{m}, \phi^{r(m)}\left(g_{\xi}\left(b_{m}\right)\right)=\phi^{r(m)}(\{\xi\})$ e,

A-ii) para cada $m<n$ e $k \in\left(E_{m+1} \backslash E_{m}\right) \cap \omega, \phi^{1-r(m)}(\{k\})=1-\phi^{1-r(m)}\left(F^{1-r(m)}\right)$.

As condições A.i) e A.ii) são satisfeitas trivialmente para $n=0$. Supondo que essas condições são satisfeitas por $n$, vai se demonstrar que $\phi^{j}$ pode se estender a $\left[E_{n+1}\right]^{<\omega}$, sendo $j \in 2$, de maneira que A.i) e A.ii) sejam satisfeitas por $n+1$.

Pela propriedade 4), o conjunto $\left\{g_{\xi}\left(b_{n}\right): \xi \in E_{m} \cap S_{r(n)}\right\} \cup\left\{\{\mu\}: \mu \in E_{n}\right\}$ é linearmente independente. De acordo com a propriedade 3), podemos estender $\phi^{r(n)} \uparrow_{\left[E_{n}\right]^{<\omega}}$ a $\left[E_{n+1}\right]^{\omega}$ de modo que para cada $\xi \in E_{n} \cap S_{r(n)}$, $\phi^{r(n)}\left(g_{\xi}\left(b_{n}\right)\right)=\phi^{r(n)}(\{\xi\})$. Então, tem-se provado que A.i) é satisfeita por $n+1$. Usando a linearidade, o homomorfismo $\phi^{1-r(n)} \uparrow_{\left[E_{n}\right]^{\omega}}$ pode ser estendido ao conjunto $\left[E_{n+1}\right]^{<\omega}$ de modo que $\phi^{1-r(n)}(\{\mu\})=1$ para cada $\mu \in E_{n+1} \backslash E_{n}$. Em particular, a condição A.ii) é satisfeita para cada $k \in\left(E_{n+1} \backslash E_{n}\right) \cap \omega$. Pela propriedade 2), ao final do processo indutivo temos que $\phi^{j}$ está definido sobre $[E]^{<\omega}$, logo, se $k \in \omega \backslash E_{0}$, então existe $n \in \omega$ tal que $k \in E_{n+1} \backslash E_{n}$; de acordo com a condição A-ii), temos que $\left(\phi^{0}(\{k\}), \phi^{1}(\{k\})\right) \neq$ $\left(\phi^{0}\left(F^{0}\right), \phi^{1}\left(F^{1}\right)\right)$, logo $\left\{k \in \omega:\left(\phi^{0}(\{k\}), \phi^{1}(\{k\})\right)=\left(\phi^{0}\left(F^{0}\right), \phi^{1}\left(F^{1}\right)\right)\right\} \subseteq E_{0} ;$ portanto, os homomorfismos $\phi^{0}$ e $\phi^{1}$ satisfazem a condição iii).

Note-se que até agora os homomorfismos $\phi^{0}$ e $\phi^{1}$ estão definidos só no conjunto $[E]^{<\omega}$, porém, qualquer homomorfismo estendendo-os vai satisfazer a condição iii), posto que $\omega \subseteq E$. Também, posto que $\phi^{0}$ e $\phi^{1}$ satisfazem i) na primeira etapa da definição, qualquer extensão deles satisfará essa propriedade.

Vejamos que para cada $j \in 2, \phi^{j}$ satisfaz a condição ii) para cada $\xi \in S_{\xi} \cap E$. Com efeito, se $\xi \in S_{\xi} \cap E$, de acordo com a definição de $E$, existe $n \in \omega$ tal que $\xi \in E_{n+1} \subseteq E_{n}$, 
mas daí por A-i), para cada $m \in r^{-1}[\{j\}] \backslash n, \phi^{j}\left(g_{\xi}\left(b_{m}\right)\right)=\phi^{j}(\{\xi\})$. Como pela condição 2), $\left\{b_{i}: i \in r^{-1}[\{j\}]\right\} \in p_{j}$, naturalmente $\left\{b_{i}: i \in r^{-1}[\{j\}] \backslash n\right\} \in p_{j}$, de modo que para cada $\xi \in S_{j} \cap E$, os homomorfismos parcialmente definidos $\phi^{0} \uparrow_{[E]^{<\omega}}$ e $\phi^{1} \uparrow_{[E]^{<\omega}}$ satisfazem ii).

Pelos comentários prévios, a construção dos homomorfismos ficará conclusa se para cada $j \in 2$, estendemos $\phi^{j} \uparrow_{[E]^{<\omega}}$ a [c] $]^{<\omega}$ de modo que a condição ii) seja satisfeita para cada $\xi \in$ $S_{j} \backslash E$.

Fixemos $j \in 2$. Seja $\gamma<\mathfrak{c}$ o menor ordinal $\xi$ para o qual $\phi^{j}(\{\xi\})$ ainda não foi definido; visto que $\bigcup_{n \in \omega} g_{\gamma}(n) \subseteq \gamma$, de acordo com a definição de $\phi$ sobre $E$, o homomorfismo $\phi^{j} \uparrow_{[E \cup \gamma]^{<\omega}}$ já está definido; portanto, é possível determinar a seqüência $\left\{\phi^{j}\left(g_{\gamma}(k)\right): k \in \omega\right\}$. Dado que $\{\gamma\} \cup\{\{\xi\}: \xi<\gamma\}$ é linearmente independente, podemos estender o homomorfismo $\phi^{j}$ ao conjunto $[E \cup \gamma+1]$ tomando

$$
\phi^{j}(\{\gamma\}) \stackrel{\text { def }}{=} p_{j}-\lim _{k \in \omega} \phi^{j}\left(g_{\gamma}(k)\right)
$$

Em conseqüência, $\left\{n \in \omega: \phi^{j}\left(g_{\gamma}(n)\right)=\phi^{j}(\{\gamma\})\right\} \in p_{j}$, logo $\phi^{j}$ satisfaz a condição ii) para $\xi=\gamma$, e, assim, para cada $\xi \in S_{j} \cap(\gamma+1)$.

Continuando o processo indutivo anterior em $\mathfrak{c} E$, definimos as funções $\phi^{0}$ e $\phi^{1}$ sobre $[\mathfrak{c}]^{<\omega}$ satisfazendo a condição ii).

Teorema 3.2.13. Sejam $p_{0}$ e $p_{1}$ ultrafiltros seletivos $\leq_{\mathrm{RK}}$-incomparáveis. Então, para cada $i \in 2$, existe um grupo topológico $G_{i} p_{i}$-compacto de modo que $G_{0} \times G_{1}$ não é enumeravelmente compacto.

Demonstração. Seja $\left\{g_{\xi}: \xi \in \mathfrak{c}\right\}$ uma enumeração de $\left([\mathfrak{c}]^{<\omega}\right)^{\omega}$ tal que para cada $\xi \in \mathfrak{c}$, $\bigcup_{n \in \omega} g_{\xi}(n) \subseteq \xi$.

Para cada $i \in 2$, seja $S_{i} \subseteq$ c tal que o conjunto

$$
\left\{\left[g_{\xi}\right]_{p_{i}}: \xi \in S_{i}\right\} \cup\left\{[\vec{\alpha}]_{p_{i}}: \alpha<\mathfrak{c}\right\}
$$

é uma base do ultraproduto $\left([\mathrm{c}]^{<\omega}\right)^{\omega} / p_{i}$.

Iniciamos por fixar a coleção $\mathscr{C}=[\mathfrak{c}]^{<\omega} \times[\mathfrak{c}]^{<\omega}$ que indexará a família de homomorfismos a partir dos quais os grupos serão construídos.

Aplicando o lema 3.2.12, no caso, considerando a seqüência $\left\{g_{\xi}: \xi<\mathfrak{c}\right\}$, os conjuntos $S_{0}$ e $S_{1}$ e cada par $\left(F_{0}, F_{1}\right) \in \mathscr{C}$, obtemos $\phi_{\left(F_{0}, F_{1}\right)}^{0}, \phi_{\left(F_{0}, F_{1}\right)}^{1} \in \operatorname{hom}\left([\mathrm{c}]^{<\omega}, 2\right)$ satisfazendo as seguintes propriedades

$\mathrm{P}$-i) se $i \in 2, \phi_{\left(F_{0}, F_{1}\right)}^{i}\left(F_{i}\right)=1$ sempre que $F_{i} \neq \varnothing$, 
P-ii) se $\xi \in S_{i},\left\{n \in \omega: \phi_{\left(F_{0}, F_{1}\right)}^{i}\left(g_{\xi}(n)\right)=\phi_{\left(F_{0}, F_{1}\right)}^{i}(\{\xi\})\right\} \in p_{i}$, e, finalmente,

P-iii) o conjunto $\left\{n \in \omega:\left(\phi_{\left(F_{0}, F_{1}\right)}^{0}(\{n\}), \phi_{\left(F_{0}, F_{1}\right)}^{1}(\{n\})\right)=\left(\phi_{\left(F_{0}, F_{1}\right)}^{0}\left(F_{0}\right), \phi_{\left(F_{0}, F_{1}\right)}^{1}\left(F_{1}\right)\right)\right\}$ é finito.

Para cada $i \in 2$, seja $H_{i} \stackrel{\text { def }}{=} \Delta \phi_{T \in \mathscr{C}} \phi_{T}^{i}:[\mathfrak{c}]^{<\omega} \rightarrow 2^{\mathscr{C}}$ a aplicação diagonal definida para cada $A \in[\mathfrak{c}]^{<\omega}$ por

$$
H_{i}(A)=\left\{\phi_{T}^{i}(A)\right\}_{T \in \mathscr{C}}
$$

ou seja, se $T \in \mathscr{C}$, satisfaz-se que $\pi_{T} \circ H_{i}=\phi_{T}^{i}$ onde $\pi_{T}: 2^{\mathscr{C}} \rightarrow 2$ representa a projeção sobre a coordenada $T$-ésima do produto $2^{\mathscr{C}}$. Como é natural, ao ser uma aplicação diagonal de homomorfismos, $H_{i}$ constitui um homomorfismo. Além disso, $H_{j}$ é injetor; de fato, para cada $F \in[c]]^{<\omega} \neq\{\varnothing\}$, o conjunto $(F, F) \in \mathscr{C}$. De acordo com a propriedade P-iii), temos que $\phi_{(F, F)}^{i}(F) \neq 0, \operatorname{logo} H_{j}(F) \neq 0 \in 2^{c}$.

Seja $G_{i} \stackrel{\text { def }}{=} \mathrm{im} H_{i} \subseteq 2^{\mathscr{C}}$ e consideremos sobre este grupo a topologia de subespaço. Afirmase que $G_{i}$ é $p_{i}$-compacto. Com efeito, se $\xi \in S_{i}$ e $M \in \mathscr{C}$, pela propriedade P-ii) temos

$$
\phi_{M}^{i}(\{\xi\})=p_{i}-\lim _{n \in \omega} \phi_{M}^{i}\left(g_{\xi}(n)\right)
$$

Considerando a seqüência em $2^{\mathscr{C}}$ dada por $\left\{H_{j}\left(g_{\xi}(n)\right): n \in \omega\right\}$, temos que

$$
\begin{aligned}
p_{i}-\lim _{n \in \omega} H_{j}\left(g_{\xi}(n)\right) & =p_{i}-\lim _{n \in \omega}\left\{\phi_{M}^{i}\left(g_{\xi}(n)\right)\right\}_{M \in \mathscr{C}} \\
& =\left\{\phi_{M}^{i}(\{\xi\})\right\}_{M \in \mathscr{C}} \\
& =H_{i}(\{\xi\})
\end{aligned}
$$

pois os $p$-limites são preservados sob funções contínuas. Como $\left\{\left[g_{\xi}\right]_{p_{i}}: \xi \in S_{i}\right\} \cup\left\{[\vec{\alpha}]_{p_{i}}: \alpha<\mathfrak{c}\right\}$ é base de $\left([c]^{<\omega}\right)^{\omega} / p_{i}$, ao considerar o homomorfismo $H_{i}$ estamos nas condições do lema3.2.7, do qual deduzimos que $H_{i}\left([\mathrm{c}]^{<\omega}\right)=G_{i}$ é $p_{i}$-compacto, isto ocorrendo para cada $i \in 2$.

Para finalizar, resta mostrar que $G_{0} \times G_{1}$ não é enumeravelmente compacto. Consideremos a seqüência $\left\{H_{0} \times H_{1}(\{n\}): n \in \omega\right\} \subseteq G_{0} \times G_{1}$; a idéia é provar que dita seqüência não tem pontos de acumulação; Com efeito, para cada par $\left(a_{0}, a_{1}\right) \in G_{0} \times G_{1}$ existe $\tilde{F}=\left(F_{0}, F_{1}\right) \in \mathscr{C}$ de modo que se $i \in 2, a_{i}=H_{i}\left(F_{i}\right)$; Notemos que o conjunto

$$
\left\{n \in \omega:\left(\pi_{\tilde{F}}\left(a_{0}\right), \pi_{\tilde{F}}\left(a_{1}\right)\right)=\pi_{\tilde{F}} \circ H_{0} \times \pi_{\tilde{F}} \circ H_{1}(\{n\})\right\}=\left\{n \in \omega:\left(\phi_{\tilde{F}}^{0}\left(F_{0}\right), \phi_{\tilde{F}}^{1}\left(F_{1}\right)\right)=\phi_{\tilde{F}}^{0} \times \phi_{\tilde{F}}^{1}(\{n\})\right\}
$$

é finito de acordo com a propriedade P-iii), $\operatorname{logo}\left(a_{1}, a_{2}\right)$ não pode ser ponto de acumulação da seqüência. Portanto, $G_{0} \times G_{1}$ não é enumeravelmente compacto. 
Corolário 3.2.14. Se existe uma família de $2^{\mathrm{C}}$ ultrafiltros seletivos $\leq_{\mathrm{RK}}$-incomparáveis, então existe uma anticadeia de comprimento $2^{\mathrm{c}}$ na $\operatorname{ordem}\left(\omega^{*}, \leq_{\mathrm{CG}}\right)$.

Demonstração. Suponhamos que $\mathscr{P}=\left\{p_{\alpha}: \alpha<2^{\mathfrak{c}}\right\}$ é uma família de ultrafiltros seletivos $\leq_{\mathrm{RK}}$-incomparáveis. Suponhamos por absurdo, que existem $p_{\alpha}, p_{\beta} \in \mathscr{P}, \alpha \neq \beta$ elementos $\leq_{\mathrm{CG}}$-compatíveis. Como $p_{\alpha}, p_{\beta}$ são $\leq_{\mathrm{RK}}$-incomparáveis, pelo teorema 3.2.13, para cada $j \in$ $\{\alpha, \beta\}$, existe um grupo topológico $G_{j}$ que é $p_{j}$-compacto, de modo que o produto $G_{\alpha} \times G_{\beta}$ não é enumeravelmente compacto. Como existe $r \in \omega^{*}$ tal que $r \leq_{C G} p_{\alpha}, p_{\beta}$, temos que $G_{\alpha}$ e $G_{\beta}$ são $r$-compactos; dado que a $r$-compacidade é produtiva, $G_{\alpha} \times G_{\beta}$ é $r$-compacto, e assim enumeravelmente compacto, uma contradição. Desta maneira, conclui-se que dois membros distintos em $\mathscr{P}$ são $\leq_{\mathrm{CG}}$-incompatíveis, ou seja, $\mathscr{P}$ é uma anticadeia na ordem $\leq_{\mathrm{CG}}$.

Corolário 3.2.15. Assumindo MA a ordem $\left(\omega^{*}, \leq_{\mathrm{CG}}\right)$ possui uma anticadeia de comprimento $2^{\mathrm{c}}$.

Demonstração. Em [12], A. Blass mostrou que MA implica a existência de $2^{\mathrm{c}}$ ultrafiltros seletivos $\leq_{\mathrm{RK}}$-incomparáveis. Pelo corolário 3.2.14, obtém-se o resultado.

Salienta-se que a construção dos homomorfismos que permitiram definir os grupos, depende das propriedades dos ultrafiltros seletivos. Aparece então uma questão natural, a saber: os ultrafiltros seletivos são necessários para obter a construção aqui apresentada?

Em relação a essa questão, o seguinte problema proposto por Tomita e Watson em [69] permanece ainda em aberto:

Questão 3.2. Se p e $q \in \omega^{*}$ são $P$-pontos $\leq_{\mathrm{RK}}$-incompatíveis, existe um grupo $p$-compacto e um grupo q-compacto cujo produto não é enumeravelmente compacto?

\subsection{Grupos de van Douwen livres e ultrafiltros seletivos}

Até agora, as topologias de grupo enumeravelmente compactas definidas sobre grupos abelianos livres usam alguma forma de MA. Uma questão natural relativa a uma solução da questão 2.2 proposta por Tkačenko, é se MA pode ser omitido nestas construções ou se definitivamente constitui uma asserção necessária.

Nesta seção, a intenção é provar que a existência de c ultrafiltros seletivos sobre $\omega^{*}$, além de produzir contraexemplos relativos à produtividade de certas propriedades em grupos topológicos como os exibidos na seção anterior, também implica a existência de uma topologia de grupo enumeravelmente compacta sobre o grupo abeliano livre de cardinalidade $\mathfrak{c}$. De acordo com os resultados de consistência que iremos explorar no que se refere a ultrafiltros seletivos, 
a construção a ser apresentada implica que MA não é uma asserção necessária para mostrar a existência de tais grupos.

\subsubsection{Codificando as seqüências em um grupo abeliano livre}

Começamos por estabelecer alguma notação. Como antes, dados um conjunto de ordinais $I$ e uma função $h \in \mathbb{Z}^{I}$, o suporte de $h$ é o conjunto supp $h=\{\alpha \in I: h(\alpha) \neq 0\}$. Seja $\bigoplus_{\alpha \in I} \mathbb{Z}=\mathbb{Z}^{(I)}=\left\{h \in \mathbb{Z}^{I}: \operatorname{supp}(h) \in[I]^{<\omega}\right\}$.

Dado $\xi \in \kappa$, definimos

$$
e_{\xi}(\mu)= \begin{cases}1, & \text { se } \mu=\xi \\ 0, & \text { se } \mu \in \kappa \backslash\{\xi\}\end{cases}
$$

Sejam $F$ e $I$ conjuntos de ordinais e $\kappa$ um cardinal. Dada uma família $\left\{z_{\xi}: \xi \in F\right\} \subseteq \mathbb{Z}^{\kappa}$ e $h \in \mathbb{Z}^{(I)}$ tal que supp $h \subseteq F$, define-se

$$
z_{h}=\sum_{\xi \in \operatorname{supp} h} h(\xi) z_{\xi}
$$

Uma família $\left\{z_{\xi}: \xi \in F\right\}$ é independente se $z_{h} \neq 0$ sempre que $h \in \mathbb{Z}^{(F)}$ e $h \neq 0$.

Se $\kappa \in \mathbb{O N}$, dada $g \in \mathbb{Z}^{(\kappa)}$ definimos

$$
\begin{aligned}
& |g|=\max \{|g(\mu)|: \mu \in \operatorname{supp} g\} \\
& \|g\|_{1}=\sum_{\mu \in \operatorname{supp} g}|g(\mu)|
\end{aligned}
$$

Pode-se verificar que tanto $|\cdot|$ quanto $\|\cdot\|_{1}$ constituem normas em $\mathbb{Z}^{(\kappa)}$.

Definição 3.3.1. Seja $\kappa \in \mathbb{O N}$. Dada uma seqüência $f \in\left(\mathbb{Z}^{(\kappa)}\right)^{\omega}$ dizemos que:

I. $f$ é do tipo I se para cada $n \in \omega,|f(n)|>n$.

II. $f$ é do tipo II se para cada $n \in \omega, \operatorname{supp} f(n) \backslash \bigcup\{\operatorname{supp} f(m): m<n\} \neq \varnothing$.

As notações $\mathscr{F}_{\text {I }}$ e $\mathscr{F}_{\text {II }}$ representam as coleções de seqüências do tipo I e do tipo II respectivamente. Denotamos por $\mathscr{F}$ o conjunto de seqüências em $\mathbb{Z}^{(\kappa)}$ do tipo I ou do tipo II, isto é, $\mathscr{F}=\mathscr{F}_{\mathrm{I}} \cup \mathscr{F}_{\text {II }}$.

Lema 3.3.2. Para cada seqüência $g \in\left(\mathbb{Z}^{(\kappa)}\right)^{\omega}$, existe $j \in \omega^{\omega}$ estritamente crescente tal que $g \circ j e ́$ constante ou $g \circ j \in \mathscr{F}$. 
Demonstração. De acordo com o conjunto $\{|g(n)|: n \in \omega\}$, temos dois casos principais a serem analisados:

i. Suponhamos que $\{|g(n)|: n \in \omega\}$ não é limitado. Então, para cada $k \in \omega$, existe $n_{k} \in \omega$ tal que $\left|g\left(n_{k}\right)\right|>k$. Note-se que a seqüência $\left\{n_{k}: k \in \omega\right\}$ pode-se tomar crescente. Tomemos $j \in \omega^{\omega}$ definindo $j(k)=n_{k}$. De acordo com a construção, claramente a seqüência $g \circ j$ pertence a $\mathscr{F}_{1}$.

ii. Suponhamos que $\{|g(n)|: n \in \omega\}$ é limitado. Aqui, surgem mais duas possibilidades:

i-a. Suponhamos que $F=\bigcup_{n \in \omega} \operatorname{supp} g(n)$ é finito. Por hipótese, existe $M \in \omega$ tal que $|g(n)| \leq M$, logo, para cada $n \in \omega$ temos $g(n) \in([-M, M] \cap \mathbb{Z})^{F} \times\{0\}^{(\ltimes \backslash F)}$. Como $F$ e $[-M, M] \cap \mathbb{Z}$ são finitos, existe $J \in[\omega]^{\omega}$ tal que para cada $m, n \in J, g(m)=g(n)$. Se enumerarmos a $J$ de maneira crescente, digamos, $J=\left\{n_{k}: k \in \omega\right\}$, definimos $j \in \omega^{\omega}$ tomando $j(k)=n_{k}$ para cada $k \in \omega$. Neste caso, temos que $g \circ j$ é constante.

i-b. Suponhamos que $F=\bigcup_{n \in \omega} \operatorname{supp} g(n)$ é infinito. Escolhemos $n_{0} \in \omega$ arbitrariamente. Com fins de fazer um processo indutivo, suponhamos que $n_{k}$ foi definido para cada $k \leq s$ de modo que supp $g\left(n_{k}\right) \backslash \bigcup_{l<k} \operatorname{supp} g\left(n_{l}\right) \neq \varnothing$. Como $F$ é infinito, e para cada $l$, supp $g_{l}$ é finito, podemos tomar $n_{s+1}=\min \left\{n>n_{s}: \operatorname{supp} g(n) \nsubseteq \bigcup_{l<k} \operatorname{supp} g\left(n_{l}\right)\right\}$. Por indução, temos então construído uma seqüência estritamente crescente $j: k \mapsto n_{k}$ de maneira que para cada $k \in \omega, \operatorname{supp} g\left(n_{k}\right) \backslash \bigcup_{l<k} \operatorname{supp} g\left(n_{l}\right) \neq \varnothing$. De acordo com a construção, é claro que $g \circ j$ pertence a $\mathscr{F}_{\text {II }}$.

\subsubsection{A construção dos homomorfismos}

Seja $\kappa \geq \mathfrak{c}$ um cardinal satisfazendo $\kappa^{\omega}=\kappa$. De maneira similar às construções já estudadas, a idéia é achar uma realização do grupo abeliano livre $\mathrm{A}(\kappa)$ como subgrupo de $\mathbb{T}^{\kappa}$ que junto com a topologia de subespaço, constituirá um grupo abeliano livre enumeravelmente compacto. Para obter uma imersão de $\mathrm{A}(\kappa)$ em $\mathbb{T}^{\kappa}$, recorremos aos dois resultados seguintes. Lema 3.3.3. Se para cada $g \in \mathbb{Z}^{(\mathrm{\kappa})} \backslash\{0\}$ existe $\phi_{g} \in \operatorname{hom}\left(\mathbb{Z}^{(\kappa)}, \mathbb{T}\right)$ tal que $\phi_{g}(g) \neq 0 \in \mathbb{T}$, então a aplicação diagonal $\Phi=\Delta\left\{\phi_{g}: g \in \mathbb{Z}^{(\kappa)} \backslash\{0\}\right\}: \mathbb{Z}^{(\kappa)} \rightarrow \mathbb{T}^{Z^{(\kappa)} \backslash\{0\}}$ é um monomorfismo algébrico.

Demonstração. Dado que para cada $g \in \mathbb{Z}^{(\kappa)}, \phi_{g} \in \operatorname{hom}\left(\mathbb{Z}^{(\kappa)}, \mathbb{T}\right), \Phi: \mathbb{Z}^{(\kappa)} \rightarrow \mathbb{T}^{Z^{(\kappa)} \backslash\{0\}}$ é um homomorfismo. Resta mostrar que o núcleo de $\Phi$ é trivial.

Para cada $f \in \mathbb{Z}^{(\kappa)}$ temos

$$
\Phi(f)=\left\{\phi_{g}(f): g \in \mathbb{Z}^{(\kappa)} \backslash\{0\}\right\}
$$


Portanto, se $f \in \mathbb{Z}^{(\kappa)}, f \neq 0 \in \mathbb{Z}^{\kappa}$, de acordo com a hipótese $\pi_{f}(\Phi(f))=\pi_{f} \circ \Phi(f)=\phi_{f}(f) \neq$ $0 \in \mathbb{T}$, isto é $\Phi(f) \neq 0$.

Lema 3.3.4. Sejam $X=\left\{x_{\xi}: \xi<\kappa\right\} \subseteq \mathbb{T}^{\kappa}, G=\langle X\rangle$ e consideremos $\mathscr{F}$ como na definição 3.3.1. Se existe uma coleção de funções $\left\{y_{f}: f \in \mathscr{F}\right\} \subseteq G$, que está fielmente indexada, tal que para cada $y \in \mathscr{F}, y_{f}$ é um ponto de acumulação de $\left\{x_{f(n)}: n \in \omega\right\}$, então $G$ é um grupo van Douwen.

Demonstração. Seja $\left\{a_{n}: n \in \omega\right\}$ uma seqüência injetora. Como $X$ gera o grupo $G$, para cada $n \in \omega$ existe uma função $g(n) \in \mathbb{Z}^{(\kappa)}$ tal que $a_{n}=x_{g(n)}$. A idéia, é mostrar que existem pelo menos dois pontos de acumulação distintos para essa seqüência; com essa finalidade, definimos para cada $i \in 2$, a subseqüência $g_{i}: \omega \rightarrow \mathbb{Z}^{(\kappa)}$ onde para cada $k \in \omega, g_{i}(k)=a_{2 k+i}=$ $x_{g(2 k+i)}$.

Pelo lema 3.3.2, para cada $i \in 2$ existe $j_{i} \in \omega^{\omega}$ de modo que $g_{i} \circ j_{i}$ é constante ou $g_{i} \circ j_{i} \in \mathscr{F}$. Se $g_{i} \circ j_{i}$ for constante, temos que a subseqüência $\left\{x_{g_{i}{ }^{\circ} j_{i}(k)}: k \in \omega\right\} \subseteq\left\{a_{2 n+i}: n \in \omega\right\}$ é constante, uma contradição. Em conseqüência, $g_{i} \circ j_{i} \in \mathscr{F}$. De acordo com a hipótese, temos que $y_{g_{i} j_{i}} \in G$ é um ponto de acumulação de $\left\{x_{g_{i} j_{i}(k)}: k \in \omega\right\}$ e, portanto, ponto de acumulação da seqüência $\left\{x_{g(n)}: n \in \omega\right\}=\left\{a_{n}: n \in \omega\right\}$, do qual deduzimos que $G$ é enumeravelmente compacto.

Notando que as funções $g_{0} \circ j_{0}$ e $g_{1} \circ j_{1} \in \mathscr{F}$ são distintas posto que a seqüência $\left\{a_{n}: n \in \omega\right\}$ é injetora, temos que $y_{g_{0} \circ j_{0}} \neq y_{g_{1} \circ j_{1}}$ são dois pontos de acumulação distintos dela. Daí, as únicas seqüências convergentes em $G$ são as triviais, isto é, $G$ é um grupo de van Douwen.

O seguinte resultado pode-se considerar como uma generalização do lema 3.2.11:

Lema 3.3.5. Suponhamos que $\left\{f_{\xi}: 0<\xi<\kappa\right\}$ constitui uma enumeração de $\mathscr{F}$ de modo tal que para cada $0<\xi<\kappa$, $\bigcup_{n \in \omega} \operatorname{supp} f_{\xi}(n) \subseteq \xi$. Seja $E \in[\kappa]^{\omega}$ tal que para cada $\xi \in E$, $\bigcup_{n \in \omega} \operatorname{supp} f_{\xi}(n) \subseteq \xi$ e tomemos $J \in \mathbb{Z}^{(\kappa)} \backslash\{0\}$ onde supp $J \subseteq E$. Se $\left\{p_{\xi}: \xi \in E\right\}$ é uma coleção de ultrafiltros seletivos $\leq_{\mathrm{RK}}$-incomparáveis, então existem uma família $\left\{E_{n}: n \in \omega\right\} \subseteq[E]^{<\omega}$, uma seqüência crescente $\left\{b_{k}: k \in \omega\right\} \subseteq \omega$, uma seqüência de reais positivos $\left\{r_{k}: k \in \omega\right\}$ e uma função $i: \omega \rightarrow$ E satisfazendo:

i. $\operatorname{supp} J \subseteq E_{0}$;

ii. $\bigcup_{n \in \omega} E_{n}=E$;

iii. $E_{k} \cup \bigcup_{m \leq k} \operatorname{supp} f_{i(m)}\left(b_{m}\right) \subseteq E_{k+1}$;

iv. para cada $k \in \omega, i(k) \in E_{k}$; 
v. para cada $\xi \in E,\left\{b_{k}: k \in i^{-1}[\xi]\right\} \in p_{\xi}$;

vi. $s e k \in \omega e f_{i(k)} \in \mathscr{F}_{\mathrm{I}},\left|f_{i(k)}\left(b_{k}\right)\right| r_{k}>2$;

vii. se $k \in \omega$ e $f_{i(k)} \in \mathscr{F}_{\mathrm{II}}, \bigcup_{n \in \omega} \operatorname{supp} f_{i(k)}(n) \backslash E_{k} \neq \varnothing$;

viii. $r_{0}=\frac{1}{4\|J\|_{1}}$, e para cada $k \in \omega, r_{k+1}=\frac{r_{k}}{2\left\|f_{i(k)}\left(b_{k}\right)\right\|_{1}}$.

Demonstração. Como no lema 3.2.11, primeiro iremos definir, indutivamente, uma seqüência $\left\{F_{n}: n \in \omega\right\} \subseteq[E]^{<\omega}$ a partir da qual definiremos posteriormente a família $\left\{E_{n}: n \in \omega\right\}$.

Seja $F_{0}:=\operatorname{supp} J$; podemos tomar $F_{1}=\bigcup_{\xi \in \text { supp } J} \operatorname{supp} f_{\xi}(0) \cup F_{0}$, e, para cada $n>1$, assumindo que temos construído $F_{n} \in[E]^{<\omega}$, é possível escolher $F_{n+1} \in[E]^{<\omega}$ satisfazendo

$$
\bigcup_{\substack{\xi \in F_{n} \\ m \leq n}} \operatorname{supp} f_{\xi}(m) \cup F_{n} \subseteq F_{n+1}
$$

Note-se que $\bigcup_{n \in \omega} F_{n} \subseteq E$. Se $\bigcup_{n \in \omega} F_{n} \neq E$, podemos redefinir $F_{0}$ para obter a igualdade.

Para cada $n \in \omega$, seja

$$
\left\||| F_{n}\right\|\|=\| J\left\|_{1}+\sum_{\substack{\xi \in F_{n} \\ m \leq n}}\right\| f_{\xi}(m) \|_{1}
$$

Seja $\xi \in E$. Para cada $n \in \omega$ definimos o conjunto

$$
A_{n}^{\xi}= \begin{cases}\left\{k \in \omega:\left|f_{\xi}(k)\right| \geq 2^{n+3}|| F_{n} \mid \|^{n+1}\right\}, & \text { se } f_{\xi} \in \mathscr{F}_{\mathrm{I}} \\ \left\{k \in \omega: \operatorname{supp} f_{\xi}(k) \backslash F_{n} \neq \varnothing\right\}, & \text { se } f_{\xi} \in \mathscr{F}_{\text {II }}\end{cases}
$$

Afirmamos que para cada $\xi \in E$ e $n \in \omega$, o conjunto $A_{n}^{\xi}$ é cofinito. Com efeito, se $f_{\xi} \in \mathscr{F}_{\text {I }}$, para cada $k \in \omega$ temos que $\left|f_{\xi}(k)\right|>k$; dai, se $M_{n}:=\left.2^{n+3}\left\|F_{n}\right\|\right|^{n+1} \in \omega$, para cada $k \geq M_{n}$, $\left|f_{\xi}(k)\right|>k \geq M_{n}$, ou seja, $\omega \backslash A_{n}^{\xi} \subseteq M_{n}$. Agora, tomemos $f_{\xi} \in \mathscr{F}_{\text {II }}$ e suponhamos por absurdo que existe $n \in \omega$ tal que $\omega \backslash A_{n}^{\xi}$ não é finito. Daí, para cada $k \in \omega$ podemos achar $m_{k} \geq k$ tal que $\operatorname{supp} f_{\xi}\left(m_{k}\right) \backslash F_{n}=\varnothing, \operatorname{logo} \bigcup_{k \in \omega} \operatorname{supp} f_{\xi}\left(m_{k}\right) \subseteq F n$, de modo que essa união é um conjunto finito. No entanto, para cada $k \in \omega, \operatorname{supp} f_{\xi}\left(m_{k}\right) \in \mathscr{F}_{\text {II }}$, de onde existe $s_{k} \in \operatorname{supp} f_{\xi}\left(m_{k}\right) \backslash \bigcup_{l<k} \operatorname{supp} f_{\xi}\left(m_{l}\right)$. Pela definição, o conjunto $\left\{s_{k}: k \in \omega\right\}$ é infinito, mas

$$
\left\{s_{k}: k \in \omega\right\} \subseteq \bigcup_{k \in \omega} \operatorname{supp} f_{\xi}\left(m_{k}\right) \subseteq F_{n},
$$

obtendo-se uma contradição. Daí, também neste caso, $\omega \backslash A_{n}^{\xi}$ é finito.

De acordo com o anterior, dado que para cada $\xi \in E$ temos $p_{\xi} \in \omega^{*}, A_{n}^{\xi} \in p_{\xi}$ para cada $n \in \omega$. 
Seja $\xi \in E$ fixo e consideremos a família $\left\{A_{n}^{\xi}: n \in \omega\right\} \subseteq p_{\xi}$. A partir dessa família será definido um elemento do ultrafiltro $p_{\xi}$.

Recursivamente, tomemos

$$
\left\{\begin{array}{l}
c_{0}^{\xi}=\min \left\{a \in A_{0}^{\xi}: a>0\right\} \\
c_{n+1}^{\xi}=\min \left\{a \in A_{n+1}^{\xi} \backslash A_{n}^{\xi}: a>\max \left\{n+1, c_{n}^{\xi}\right\}\right\}
\end{array}\right.
$$

Tomemos $C_{0}=\left[0, c_{0}^{\xi}\right]$, e, para cada $n \in \omega$, seja $C_{n+1}=\left[c_{n}^{\xi}+1, c_{n+1}^{\xi}\right]$. De acordo com a definição da seqüência $\left\{c_{n}^{\xi}: n \in \omega\right\}$, observemos que $\left\{C_{n}: n \in \omega\right\}$ constitui uma partição de $\omega$. Como $p_{\xi}$ é seletivo, para cada $n \in \omega$, existe $a_{n}^{\xi} \in C_{n}$ de modo que $\left\{a_{n}^{\xi}: n \in \omega\right\} \in p_{\xi}$. Para cada $n \in \omega$, observemos que $a_{n}^{\xi} \geq n$ e $a_{n}^{\xi} \in A_{n}^{\xi}$.

Considerando a seqüência $\left\{a_{n}^{\xi}: n \in \omega\right\} \in p_{\xi}$, dado que $|E|=\omega$, de acordo com o lema 3.2.10 existe uma família $\left\{I_{\xi}: \xi \in E\right\}$ de subconjuntos de $\omega$ tal que

a. para cada $\xi \in E,\left\{a_{k}^{\xi}: k \in I_{\xi}\right\} \in p_{\xi}$;

b. $\left\{\left[k, a_{k}^{\xi}\right]: \xi \in E, k \in I_{\xi}\right\}$ é uma família de intervalos de $\omega$ dois a dois disjuntos.

Para cada $\xi \in E$, seja $N_{\xi}=\min \left\{n \in \omega: \xi \in F_{n}\right\}$. Notando que qualquer subconjunto de $I_{\xi}$ satisfaz a condição ii) e cada $I_{\xi}$ é infinito, sem perda de generalidade pode-se assumir que para cada $k \in I_{\xi}, k>N_{\xi}$.

Sejam $\xi, \eta \in E$. Pela propriedade b), para quaisquer $k \in I_{\xi}, l \in I_{\eta}, \quad\left[k, a_{k}^{\xi}\right] \cap\left[l, a_{l}^{\eta}\right]=\varnothing$, o que implica que $I_{\xi} \cap I_{\eta}=\varnothing$. Daí, a família $\left\{I_{\xi}: \xi \in E\right\}$ está formada por conjuntos dois a dois disjuntos. Como $\bigcup_{\xi \in E} I_{\xi}$ é enumerável, podemos tomar uma enumeração crescente $\left\{n_{k}: k \in \omega\right\}$ desse conjunto; de acordo com isto, para cada $k \in \omega$ existe um único $i(k) \in E$ tal que $n_{k} \in I_{i(k)}$, com o qual temos definimos uma função $i: \omega \rightarrow E$.

Para cada $k \in \omega$, sejam

$$
b_{k}:=a_{n_{k}}^{i(k)}, \quad E_{k}:=F_{n_{k}}
$$

Como tem-se definido a seqüência $\left\{b_{k}: k \in \omega\right\}$ e a função $i: \omega \rightarrow E$, procedemos a definir a seqüência de reais positivos $\left\{r_{k}: k \in \omega\right\}$ recursivamente:

$$
\begin{aligned}
r_{0}: & =\frac{1}{4\|J\|_{1}} \\
r_{k+1} & =\frac{r_{k}}{2\left\|f_{i(k)}\left(b_{k}\right)\right\|_{1}}
\end{aligned}
$$

A seguir, mostramos que a família $\left\{E_{k}: k \in \omega\right\}$, as seqüências $\left\{b_{k}: k \in \omega\right\} \subseteq \omega \mathrm{e}$ $\left\{r_{k}: k \in \omega\right\} \subseteq \mathbb{R}$ e a função $i: \omega \rightarrow E$ satisfazem as condições i)-vii): 
i. Temos que supp $J \subseteq F_{0} \subseteq F_{n_{0}}=E_{0}$.

ii. Como $\left\{n_{k}: k \in \omega\right\}$ é crescente, temos que para cada $k \in \omega, k \leq n_{k}$; daí, pela construção da família $\left\{F_{n}: n \in \omega\right\}, F_{k} \subseteq F_{n_{k}}$ e assim

$$
E=\bigcup_{n \in \omega} F_{k} \subseteq \bigcup_{k \in \omega} F_{n_{k}}=\bigcup_{n \in \omega} E_{k}
$$

iii. Para cada $k \in \omega, n_{k}<n_{k+1}$, logo $F_{n_{k}} \subseteq F_{n_{k+1}}$ isto é, $E_{k} \subseteq E_{k+1}$. Mostraremos que para cada $m \leq k, \operatorname{supp} f_{i(m)}\left(b_{m}\right) \subseteq E_{k+1}$. Pela construção da família $\left\{F_{n}: n \in \omega\right\}$, note-se que

$$
\begin{aligned}
E_{k+1}=F_{n_{k+1}} & \supseteq \bigcup\left\{\operatorname{supp} f_{\xi}(m): m \leq n_{k+1}, \xi \in F_{n_{k+1}}\right\} \\
& \supseteq \bigcup\left\{\operatorname{supp} f_{\xi}(m): m \leq n_{k+1}-1, \xi \in F_{n_{k+1}-1}\right\}
\end{aligned}
$$

Se supomos que a condição iv) é satisfeita, temos que para cada $m \leq k, i(m) \in E_{m} \subseteq E_{k}=$ $F_{n_{k}} \subseteq F_{n_{k+1}-1}$; pela condição b), temos que $n_{m}<n_{k+1}$ implica $\left[n_{m}, a_{n_{m}}^{i(m)}\right] \cap\left[n_{k+1}, a_{n_{k+1}}^{i(k+1)}\right]=\varnothing$, isto é, $b_{m}=a_{n_{m}}^{i(m)} \leq n_{k+1}-1$. Dado que $i(m) \in F_{n_{k+1}-1}$ e $b_{m} \leq n_{k+1}-1$, o conjunto $\operatorname{supp} f_{i(m)}\left(b_{m}\right)$ está contido em $(*)$; portanto, $\operatorname{supp} f_{i(m)}\left(b_{m}\right) \subseteq E_{k+1}$.

iv. Seja $k \in \omega$. Por definição, $b_{k}=a_{n_{k}}^{i(k)}$ e $n_{k} \in I_{i(k)}$ de onde $n_{k}>N_{i(k)}$. Note-se então que $i(k) \in F_{N_{i(k)}} \subseteq F_{n_{k}}=E_{k}$.

v. De acordo com a propriedade a), para cada $\xi \in E$,

$$
\begin{aligned}
\left\{b_{k}: k \in i^{-1}[\{\xi\}]\right\} & =\left\{a_{n_{k}}^{i(k)}: k \in i^{-1}[\{\xi\}]\right\} \\
& =\left\{a_{n_{k}}^{i(k)}: n_{k} \in I_{\xi}\right\} \in p_{\xi}
\end{aligned}
$$

vi. Seja $k \in \omega$ e $f_{i(k)} \in \mathscr{F}_{1}$. Para cada $m<k$, temos que $\left[m, a_{n_{m}}^{i(m)}\right] \cap\left[k, a_{n_{k}}^{i(k)}\right]=\varnothing$, logo $b_{m}=a_{n_{m}}^{i(m)}<k \leq n_{k}$. 
Da definição da seqüência $\left\{r_{k}: k \in \omega\right\}$, temos que

$$
\begin{aligned}
r_{0} & =\frac{1}{4\|J\|_{1}} \\
r_{1} & =\frac{r_{0}}{2\left\|f_{i(0)}\left(b_{0}\right)\right\|_{1}}=\frac{1}{4\|J\|_{1}}\left(\frac{1}{2\left\|f_{i(0)}\left(b_{0}\right)\right\|_{1}}\right) \\
r_{2} & =\frac{r_{1}}{2\left\|f_{i(1)}\left(b_{1}\right)\right\|_{1}} \\
& =\frac{1}{4\|J\|_{1}}\left(\frac{1}{2\left\|f_{i(0)}\left(b_{0}\right)\right\|_{1}}\right)\left(\frac{1}{2\left\|f_{i(1)}\left(b_{1}\right)\right\|_{1}}\right) \\
& =\frac{1}{4\|J\|_{1}}\left(\frac{1}{2^{2}\left\|f_{i(0)}\left(b_{0}\right)\right\|_{1}\left\|f_{i(1)}\left(b_{1}\right)\right\|_{1}}\right)
\end{aligned}
$$

Indutivamente, pode-se provar que para cada $k \geq 1$,

$$
r_{k}=\frac{1}{4\|J\|_{1}}\left(\frac{1}{2^{k}} \prod_{m<k} \frac{1}{\left\|f_{i(m)}\left(b_{m}\right)\right\|_{1}}\right)
$$

Lembre que $b_{k}=a_{n_{k}}^{i(k)} \in A_{n_{k}}^{i(k)}$, logo por definição $\left|f_{i(k)}\left(b_{k}\right)\right| \geq 2^{n_{k}+3}||\left|F_{n_{k}} \|\right|^{n_{k+1}}$. Daí,

$$
\begin{aligned}
\left|f_{i(k)}\left(b_{k}\right)\right| r_{k} & \geq \frac{2^{n_{k}+3}\left\|\mid F_{n_{k}}\right\|^{n_{k}+1}}{4\|J\|_{1} 2^{k}}\left(\prod_{m<k} \frac{1}{\left\|f_{i(m)}\left(b_{m}\right)\right\|_{1}}\right) \\
& =\frac{2^{n_{k}+1}}{2^{k}} \frac{\left\|F_{n_{k}}\right\| \|^{n_{k}-k+1}}{\|J\|_{1}}\left(\prod_{m<k} \frac{\left\|F_{n_{k}}\right\|}{\left\|f_{i(m)}\left(b_{m}\right)\right\|_{1}}\right) \\
& >2 \frac{\left\|F_{n_{k}}\right\| \|}{\|J\|_{1}} \prod_{m<k} \frac{\left\|F_{n_{k}}\right\|}{\left\|f_{i(m)}\left(b_{m}\right)\right\|_{1}} \quad(* *)
\end{aligned}
$$

Dado que

$$
\left\||| F_{n_{k}} \mid\right\|=\|J\|_{1}+\sum_{\substack{\xi \in F_{n_{k}} \\ m \leq n_{k}}}\left\|f_{\xi}(m)\right\|_{1}
$$

tem-se $\left\|F_{n_{k}} \mid\right\| \geq\|J\|_{1}$, ou seja $\frac{\left\|F_{n_{k}}\right\|}{\|J\|_{1}} \geq 1$; ora bem, a condição iv) implica que para cada $m<k, i(m) \in E_{m}=F_{n_{m}} \subseteq F_{n_{k}}$, logo $\left\|F_{n_{k}}\right\| \geq\left\|f_{i(m)}\left(b_{m}\right)\right\|_{1}$, isto é, $\frac{\left\|F_{n_{n}}\right\|}{\left\|f_{i(m)}\left(b_{m}\right)\right\|_{1}} \geq 1$. Consequentemente, em $(* *)$ tem-se $\left|f_{i(k)}\left(b_{k}\right)\right| r_{k}>2$ para cada $k \leq 1$.

vii. Sejam $k \in \omega$ e $f_{i(k)} \in \mathscr{F}_{\text {II }}$. Como $b_{k}=a_{n_{k}}^{i(k)} \in A_{n_{k}}^{i(k)}$, de acordo com a definição, temos que $\operatorname{supp} f_{i(k)}\left(b_{k}\right) \backslash F_{n_{k}} \neq \varnothing$, isto é, $\operatorname{supp} f_{i(k)}\left(b_{k}\right) \backslash E_{k} \neq \varnothing$.

Definição 3.3.6. Consideremos o grupo do círculo $\mathbb{T}$. Note que de acordo com o exemplo 1.3.15, podemos identificar $\mathbb{T}$ com o grupo quociente $\mathbb{R} / \mathbb{Z}$. Para cada $z=r+\mathbb{Z} \in \mathbb{T}$, definimos

$$
\|z\|_{\mathbb{T}}=\|r+\mathbb{Z}\|_{\mathbb{T}}=d(r, \mathbb{Z})=\min \{|r+m|: m \in \mathbb{Z}\}
$$


onde $d$ representa a distância usual em $\mathbb{R}$. Seja $\delta: \mathbb{T} \times \mathbb{T} \rightarrow \mathbb{R}$ a métrica gerada pela norma $\|\cdot\|_{\mathbb{T}} ; \operatorname{assim} \operatorname{dados} r+\mathbb{Z}, s+\mathbb{Z} \in \mathbb{T}$,

$$
\delta(r+\mathbb{Z}, s+\mathbb{Z})=\|r-s+\mathbb{Z}\|_{\mathbb{T}}=\min \{|r-s+m|: m \in \mathbb{Z}\}
$$

É fácil ver que a topologia induzida por $\delta$ sobre $\mathbb{T}$, coincide com a topologia usual desse espaço.

Dado $A \subseteq \mathbb{T}, \delta(A)$ representa o diâmetro de $A$ na métrica $\delta$, isto é

$$
\delta(A)=\sup \{\delta(a, b): a, b \in A\}
$$

Seja $\mathcal{B}$ a coleção de todos os arcos abertos em $\mathbb{T}$, supondo convenientemente que $\mathbb{T} \in \mathcal{B}$. Se $E \in[\kappa]^{\omega}$, dados uma função $\psi: E \rightarrow \mathcal{B}$ e um conjunto $H \in \mathbb{Z}^{(E)}$, definimos

$$
\psi(H)=\sum_{\mu \in \operatorname{supp} H} H(\mu) \psi(\mu)
$$

Lema 3.3.7. Sejam $E \in[\kappa]^{\omega}, A \in[E]^{<\omega}$ e $r \in \mathbb{R}, 0<r<\frac{1}{2}$. Suponhamos que $\psi: E \rightarrow \mathcal{B} e ́$ uma função tal que:

- $A=\{\xi \in E: \psi(\xi) \neq \mathbb{T}\}$

- para cada $\xi \in A, \delta(\psi(\xi))=r$

Sejam $\mu \in A$ e $H \in \mathbb{Z}^{(E)}$ tais que $\mu \notin \operatorname{supp} H$; suponha-se, adicionalmente, que alguma das seguintes duas condições é satisfeita

a) se supp $H \subseteq A$, então $|H| r>2$, ou

b) $\operatorname{supp} H \backslash A \neq \varnothing$,

Então, existe $\psi^{*}: E \rightarrow \mathcal{B}$ satisfazendo

i) para cada $\xi \in E, \overline{\psi^{*}(\xi)} \subseteq \psi(\xi)$;

ii) $s e \xi \in A \cup \operatorname{supp} H, \delta\left(\psi^{*}(\xi)\right)=\frac{r}{2\|H\|_{1}}$. Em outro caso, $\psi^{*}(\xi)=\mathbb{T}$;

iii) $\psi^{*}(\mu) \cap\left(\sum_{\xi \in \operatorname{supp} H} H(\xi) \psi(\xi)\right) \neq \varnothing$. 
Demonstração. Notemos que $E=E \backslash(\operatorname{supp} H \cup A) \cup(\operatorname{supp} H \cup A)$. Vamos definir $\psi^{*}$ em cada uma dessas partes disjuntas.

Para cada $\xi \in A \backslash \operatorname{supp} H, \psi(\xi) \neq \mathbb{T}$. Por hipótese, notemos que $\delta(\psi(\xi))=r$, logo é possível achar $\psi^{*}(\xi) \in \mathcal{B}$ de modo que $\delta\left(\psi^{*}(\xi)\right)=\frac{r}{2\|H\|_{1}}$ e $\overline{\psi^{*}(\xi)} \subseteq \psi(\xi)$.

Agora, se $\xi \in E \backslash(\operatorname{supp}(H) \cup A)$, para satisfazer ii), convenientemente tomamos $\psi^{*}(\xi)=\mathbb{T}$.

Por último, vamos centrar a nossa atenção aos elementos de supp $H$. Se $H$ é como no caso a), isto é, supp $H \subseteq A$ e $|H| r>2$, de acordo com a definição da norma, existe $\alpha \in \operatorname{supp} H$ tal que $|H(\alpha)| r>2$, ou seja, $|H(\alpha)| \frac{1}{2}>\frac{1}{r}>1$. Como $\delta(\psi(\alpha))=r$, notemos então que $H(\alpha) \psi(\alpha)=\mathbb{T}$. De outra parte, se $H$ for como no caso b), podemos escolher $\alpha \in \operatorname{supp} H \backslash A$. Por hipótese, temos que $\psi(\alpha)=\mathbb{T}, \operatorname{logo} H(\alpha) \psi(\alpha)=H(\alpha) \mathbb{T}=\mathbb{T}$.

Em qualquer dos casos anteriores, para cada $\xi \in \operatorname{supp} H \backslash\{\alpha\}$, fixemos $y_{\xi} \in \psi(\xi)$. Como $\mu \in A \backslash \operatorname{supp} H$, repare-se que $\psi^{*}(\mu)$ já está definido, e, além disso, temos que $H(\alpha) \psi(\alpha)=\mathbb{T}$. Daí, tomando um elemento arbitrário $k \in \psi^{*}(\mu)$, podemos escolher $y_{\alpha} \in \psi(\alpha)$ de modo que $H(\alpha) y_{\alpha}=\underset{\xi \in \operatorname{supp} H \backslash\{\alpha\}}{k-\sum_{\xi} H(\xi)} y_{\xi}$, isto é

$$
H(\alpha) y_{\alpha}+\sum_{\xi \in \operatorname{supp} H \backslash\{\alpha\}} H(\xi) y_{\xi} \in \psi^{*}(\mu)
$$

No caso a), para cada $\xi \in \operatorname{supp} H$ notemos que $\delta(\psi(\xi))=r$. Como $y_{\xi} \in \psi(\xi)$, podemos achar $\psi^{*}(\xi) \in \mathcal{B}$ de modo que $\delta\left(\psi^{*}(\xi)\right)=\frac{r}{2\|H\|_{1}}$ e $\overline{\psi^{*}(\xi)} \subseteq \psi(\xi)$. No caso b), se $\xi \in \operatorname{supp} H \cap A$, é possível escolher $\psi^{*}(\xi)$ como acima; agora, se $\xi \in \operatorname{supp} H \backslash A$, visto que $\psi(\xi)=\mathbb{T}$, escolhemos de maneira conveniente $\psi^{*}(\xi)$ contendo a $y_{\xi}$, de modo que a condição ii) seja satisfeita.

Por $(\star)$, temos $\sum_{\xi \in \text { supp } H} H(\xi) y_{\xi} \in \psi^{*}(\mu)$ e, em virtude da construção, se satisfaz

$$
\sum_{\xi \in \operatorname{supp} H} H(\xi) y_{\xi} \in \sum_{\xi \in \operatorname{supp} H \backslash\{\alpha\}} H(\xi) \psi_{\xi}
$$

Em conseqüência, $\psi^{*}(\mu) \cap \psi(H) \neq \varnothing$.

Lema 3.3.8. Sejam $\left\{f_{\xi}: 0<\xi<\kappa\right\}, J \in \mathbb{Z}^{(\kappa)} \backslash\{0\}$ e $E \in[\kappa]^{<\omega}$ como na hipótese do lema 3.3.5. Se $\left\{p_{\xi}: \xi \in E\right\}$ é uma família de ultrafiltros seletivos $\leq_{\mathrm{RK}}$-incomparáveis, então existe um homomorfismo $\phi: \mathbb{Z}^{(E)} \rightarrow \mathbb{T}$ satisfazendo:

1. $\phi(J) \neq 0 \in \mathbb{T}$;

2. para cada $\xi \in E, p_{\xi}-\lim _{n \in \omega} \phi\left(f_{\xi}(n)\right)=\phi\left(\chi_{\xi}\right)$.

Demonstração. Como estamos assumindo as hipóteses do lema 3.3.5, podemos supor que existem uma família $\left\{E_{n}: n \in \omega\right\} \subseteq[E]^{<\omega}$, seqüências $\left\{b_{k}: k \in \omega\right\} \subseteq \omega,\left\{r_{k}: k \in \omega\right\} \subseteq \mathbb{R}^{>0}$ 
e uma função $i: \omega \rightarrow E$ satisfazendo as condições i)-viii) do referido lema. Como antes, $\mathcal{B}$ representa a coleção de todos os arcos abertos de $\mathbb{T}$, incluindo o próprio $\mathbb{T}$. Definiremos por indução, funções $\psi_{n}: E \rightarrow \mathcal{B}$ que permitem construir o homomorfismo $\phi$.

Na etapa 0) da indução, para cada $\xi \in E_{0}$ escolha $y_{\xi} \in \mathbb{R}$ tal que $\sum_{\xi \in \text { supp } J} J(\xi) y_{\xi}=\frac{1}{2}$. Seja

$$
\psi_{0}(\xi)=\pi\left[\left(y_{\xi}-\frac{r_{0}}{2}, y_{\xi}+\frac{r_{0}}{2}\right)\right]
$$

Note-se que

$$
\begin{aligned}
\delta\left(\psi_{0}(J)\right)=\delta\left(\sum_{\xi \in \operatorname{supp} J} J(\xi) \Psi_{0}(\xi)\right) & =\delta\left(\sum_{\xi \in \operatorname{supp} J} J(\xi) \pi\left[\left(y_{\xi}-\frac{r_{0}}{2}, y_{\xi}+\frac{r_{0}}{2}\right)\right]\right) \\
& \leq \sum_{\xi \in \operatorname{supp} J} \delta\left(J(\xi) \pi\left[\left(y_{\xi}-\frac{r_{0}}{2}, y_{\xi}+\frac{r_{0}}{2}\right)\right]\right) \\
& \leq \sum_{\xi \in \operatorname{supp} J}|J(\xi)| r_{0}=\|J\|_{1} r_{0}=\frac{\|J\|_{1}}{4\|J\|_{1}}=\frac{1}{4}
\end{aligned}
$$

Para cada $\xi \in \operatorname{supp} J$, claramente $J(\xi) y_{\xi}+\mathbb{T} \in \psi_{0}(\xi)$. Daí,

$$
\sum_{\xi \in \operatorname{supp}}\left(J(\xi)+y_{\xi}+\mathbb{T}\right)=\frac{1}{2}+\mathbb{T} \in \psi_{0}(J)
$$

observemos que $\delta\left(\psi_{0}(J)\right) \leq \frac{1}{4}$, logo $0 \notin \overline{\psi_{0}(J)}$.

Suponhamos que para cada $k \leq m$, temos definido $\psi_{k}: E \rightarrow \mathcal{B}$ satisfazendo

A-i) para cada $\xi \in E$ e $k<m, \overline{\psi_{k+1}(\xi)} \subseteq \psi_{k}(\xi)$;

A-ii) para cada $k \leq m$, se $\xi \in E_{k}, \delta\left(\psi_{k}(\xi)\right)=r_{k}$ e, se $\xi \in E \backslash E_{k}, \psi_{k}(\xi)=\mathbb{T}$;

A-iii) para cada $k<m, \psi_{k+1}(i(k)) \cap \sum_{\psi_{k+1}}\left(f_{i(k)}\left(b_{k}\right)\right) \neq \varnothing$.

Como para cada $k \in \omega$ temos supp $f_{i(k)}(k) \subseteq i(m)$ segue $i(m) \notin \operatorname{supp} f_{i(k)}\left(b_{m}\right)$. Daí, tomando $A=E_{m}, \psi=\psi_{m}, H=f_{i(m)}\left(b_{m}\right) \mu=i(m)$ e $r=r_{m}$, pelo teorema 3.3.7, existe $\psi^{*}: E \rightarrow \mathcal{B}$ satisfazendo

B-i) para cada $\xi \in E, \overline{\psi^{*}(\xi)} \subseteq \psi_{m}(\xi)$;

B-ii) para cada $\xi \in E_{m} \cup \operatorname{supp} f_{i(m)}\left(b_{m}\right), \delta\left(\psi^{*}(\xi)\right)=\frac{r_{m}}{2\left\|f_{i(m)}\left(b_{m}\right)\right\|_{1}}=r_{m+1}$ e, em outro caso, $\psi^{*}(\xi)=$ $\mathbb{T}$;

B-iii) $\psi^{*}(i(m)) \cap \psi^{*}\left(f_{i(m)}\left(b_{m}\right)\right) \neq \varnothing$. 
Se $\xi \in E_{m} \cup \operatorname{supp} f_{i(m)}\left(b_{m}\right) \cup\left(E \backslash E_{m+1}\right)$, tome $\psi_{m+1}(\xi)=\psi^{*}(\xi)$. Se $\xi \in E_{m+1} \backslash\left(E_{m} \cup \operatorname{supp} f_{i(m)}\left(b_{m}\right)\right)$, escolhemos $\psi_{m+1}(\xi) \in \mathcal{B}$ convenientemente para que $\overline{\psi_{m+1}(\xi)} \subseteq \psi_{m}(\xi)$ e $\delta\left(\psi_{m+1}(\xi)\right)=r_{m+1}$. Claramente, esta escolha implica que $\psi_{m+1}$ satisfaz as hipóteses indutivas A-i)-A-iii).

Fixemos $\xi \in E$. Pelas condições B-i) e B-ii), temos que $\left\{\psi_{k}(\xi): k \in \omega\right\}$ é uma seqüência decrescente de conjuntos fechados no compacto $\mathbb{T}$, logo $\bigcap_{k \in \omega} \overline{\psi_{k}(\xi)}=\bigcap_{k \in \omega} \psi_{k}(\xi) \neq \varnothing$.

Note-se que $\lim _{k \in \omega} \delta\left(\psi_{k}(\xi)\right)=\lim _{k \in \omega} r_{k}=0$; de fato, dado $0<\varepsilon<r_{0}$, seja $m \in \omega$ tal que $\frac{1}{b_{m}}<\varepsilon$ e $f_{i(m)} \in \mathscr{F}_{\mathrm{I}}$. Como $\left|f_{i(m)}\left(b_{m}\right)\right|>b_{m}$, tem-se $\frac{1}{\left\|f_{i(m)}\left(b_{m}\right)\right\|_{1}}<\varepsilon$. Observemos que $r_{k} \leq r_{0}=\frac{1}{4\|J\|_{1}}$ implica que $\frac{r_{k}}{2 \| f_{i(m)\left(b_{m}\right) \|_{1}}}=r_{k+1}<\varepsilon$. Portanto, existe $w_{\xi} \in \mathbb{T}$ tal que $\bigcap_{k \in \omega} \psi_{k}(\xi)=\left\{w_{\xi}\right\}$.

Ocorrendo isto para cada $\xi \in E$, define-se $\phi\left(\chi_{\xi}\right)=w_{\xi}$. Como $\mathbb{Z}^{(E)}=\left\langle\chi_{\xi}: \xi \in E\right\rangle$, podemos estender $\phi$ a um homomorfismo $\phi: \mathbb{Z}^{(E)} \rightarrow \mathbb{T}$. A continuação, provaremos que $\phi$ satisfaz as condições 1) e 2).

Note-se que

$$
\phi(J)=\sum_{\xi \in \text { supp } J} J(\xi) \omega_{\xi} \in \sum_{\xi \in \text { supp } J} J(\xi) \psi_{0}(\xi)=\psi_{0}(J)
$$

Visto que $0 \notin \overline{\psi_{0}(J)}$, tem-se $\phi(J) \neq 0$.

Agora, consideremos a seqüência $\left\{\phi\left(f_{\xi}(n)\right): n \in \omega\right\} \subseteq \mathbb{T}$ e seja $k \in i^{-1}(\{\xi\})$. Notemos que $\operatorname{supp} f_{\xi}\left(b_{k}\right) \subseteq E_{k+1}$. Pela definição de $\phi$, para cada $\eta \in \operatorname{supp} f_{\xi}\left(b_{k}\right), w_{\eta} \in \psi_{k+1}(\eta)$. Daí, como

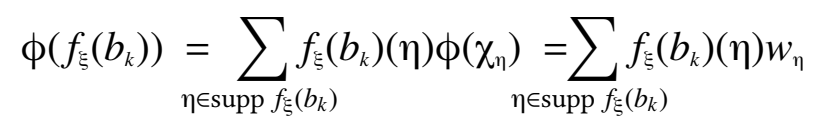

temos que $\phi\left(f_{\xi}\left(b_{k}\right)\right) \in \psi_{k+1}\left(f_{\xi}\left(b_{k}\right)\right)=\sum_{\eta \in \operatorname{supp}} f_{\xi_{\xi}\left(b_{k}\right)} f_{\xi}\left(b_{k}\right)(\eta) \psi_{k+1}(\eta)$

De outra parte, $w_{\xi}=\phi\left(\chi_{\xi}\right) \in \psi_{k+1}(\xi)$. Pela condição iii), podemos tomar também $z_{\xi}^{k} \in \psi_{k+1}(\xi) \cap \psi_{k+1}\left(f_{\xi}\left(b_{k}\right)\right)$; este elemento proporciona uma estimativa da distância entre $\phi\left(\chi_{\xi}\right)$ e $\phi\left(f_{\xi}\left(b_{k}\right)\right)$. De fato, observamos

$$
\begin{aligned}
\delta\left(\phi\left(\chi_{\xi}\right), \phi\left(f_{\xi}\left(b_{k}\right)\right)\right) & \leq \delta\left(\phi\left(\chi_{\xi}\right), z_{\xi}^{k}\right)+\delta\left(z_{\xi}^{k}, \phi\left(f_{\xi}\left(b_{k}\right)\right)\right) \\
& \leq \delta\left(\psi_{k+1}(\xi)\right)+\delta\left(\psi_{k+1}\left(f_{\xi}\left(b_{k}\right)\right)\right) \\
& \leq r_{k+1}+\sum_{\eta \in \operatorname{supp} f_{\xi}\left(b_{k}\right)}\left|f_{\xi}\left(b_{k}\right)(\eta)\right| \delta\left(\psi_{k+1}(\xi)\right) \\
& \leq r_{k+1}+\sum_{\eta \in \operatorname{supp} f_{\xi}\left(b_{k}\right)}\left|f_{\xi}\left(b_{k}\right)(\eta)\right| r_{k+1} \\
& =r_{k+1}+r_{k+1}\left(\left\|f_{\xi}\left(b_{k}\right)\right\|_{1}\right) \\
& =r_{k+1}\left(1+\left\|f_{\xi}\left(b_{k}\right)\right\|_{1}\right) \\
& =\frac{r_{k}}{2\left\|f_{\xi}\left(b_{k}\right)\right\|_{1}}\left(1+\left\|f_{\xi}\left(b_{k}\right)\right\|_{1}\right) \leq r_{k}
\end{aligned}
$$


Como $\lim _{n \rightarrow \infty} r_{n}=0$, a subseqüência $\left\{r_{k}: k \in i^{-1}[\{\xi\}]\right\}$ também tem como limite o ponto 0 o que pela sua vez implica a convergência ao ponto $\phi\left(\chi_{\xi}\right)$ da seqüência $\left\{\phi\left(f_{\xi}\left(b_{k}\right)\right): k \in\right.$ $\left.i^{-1}[\{\xi\}]\right\}$. Portanto, dado um aberto $U \subseteq \mathbb{T}$ contendo o ponto $\phi\left(\chi_{\xi}\right)$, existe $k_{0} \in i^{-1}[\{\xi\}]$ tal que $\left\{\phi\left(f_{\xi}\left(b_{k}\right)\right): k \geq k_{0}, k \in i^{-1}[\{\xi\}]\right\} \subseteq U$, isto é

$$
\left\{b_{k}: k \in i^{-1}[\{\xi\}], \phi\left(f_{\xi}\left(b_{k}\right)\right) \in U\right\}=\left\{b_{k}: k \in i^{-1}[\{\xi\}], k \geq k_{0}\right\} \in p_{\xi}
$$

ou seja, $p_{\xi}-\lim _{k \in \omega} \phi\left(f_{\xi}\left(b_{k}\right)\right)=\phi\left(\chi_{\xi}\right)$, obtendo-se o resultado.

Lema 3.3.9. Seja $\left\{f_{\xi}: 0<\xi<\kappa\right\}$ uma enumeração de $\mathscr{F}$ tal que para cada $0<\xi<\kappa$, $\bigcup \operatorname{supp} f_{\xi}(n) \subseteq \xi$. Se $J \in \mathbb{Z}^{(\kappa)} \backslash\{0\}$, então existe $E \in[\kappa]^{\omega}$ tal que para cada $\xi \in E \backslash\{0\}$, $\bigcup_{n \in \omega}^{n \in \omega} \operatorname{supp} f_{\xi}(n) \subseteq E$.

Demonstração. Definimos por indução $E^{0}=\{0\}$. Para cada $0<\xi<\kappa$, seja

$$
E^{\xi}=\{\xi\} \cup \bigcup\left\{E^{\mu}: \mu \in \bigcup_{n \in \omega} \operatorname{supp} f_{\xi}(n)\right\}
$$

Notemos que para cada $0<\xi<\kappa$, o conjunto $\bigcup_{n \in \omega} \operatorname{supp} f_{\xi}(n) \subseteq \xi$ é enumerável, logo, dado que supp $J$ é finito, definindo $E=\bigcup_{\xi \in \text { supp } J} E^{\xi} \subseteq \kappa$ temos que $E \in[\kappa]^{\omega}$. Sejam $\xi \in E \backslash\{0\}$ e $n \in \omega$; pela definição, existe $\eta \in \operatorname{supp} J$ tal que $\xi \in E^{\eta}$; de acordo com a definição de $E^{\eta}$, temos que $\xi=\eta$ ou existe $\alpha \in \bigcup_{n \in \omega} \operatorname{supp} f_{\xi}(n) \subseteq \xi$ tal que $\xi \in E^{\alpha}$. No primeiro caso, dado que para cada $0<\mu<\kappa$ tem-se $\mu \in E^{\mu}$, obtemos $\bigcup_{n \in \omega} \operatorname{supp} f_{\xi}(n) \subseteq E^{\xi} \subseteq E$. No segundo caso, observe que $\xi \in E^{\alpha_{1}}$ para algum $\alpha_{1} \in \bigcup_{n \in \omega} \operatorname{supp} f_{\eta}(n) \subseteq \eta$. Se $\xi=\alpha_{1}$, temos o resultado. Se não, existe $\alpha_{2} \in \bigcup_{n \in \omega} \operatorname{supp} f_{\alpha_{1}}(n) \subseteq \alpha_{1}$ de modo que $\xi \in E^{\alpha_{2}}$. Continuando desta maneira, achamos ordinais $\eta>\alpha_{1}>\alpha_{2}>\cdots>\alpha_{k}>\cdots$ tais que para cada $i, \alpha_{i+1} \in \bigcup_{n \in \omega} \operatorname{supp} f_{\alpha_{i}}(n)$ e $\xi \in E^{\alpha_{i}}$. Note que essa seqüência não pode ser infinita, $\operatorname{logo} \alpha_{k_{0}}=\xi$ para algum $k_{0} \in \omega$; daí, $E^{\xi} \subseteq E^{\eta} \subseteq E$ e $\bigcup_{n \in \omega} \operatorname{supp} f_{\xi}(n) \subseteq E^{\xi}$.

Lema 3.3.10. Seja $\left\{f_{\xi}: 0<\xi<\kappa\right\}$ uma enumeração de $\mathscr{F}$ de modo tal que para cada $0<\xi<\kappa$, $\bigcup_{n \in \omega} \operatorname{supp} f_{\xi_{1}}(n) \subseteq \xi$. Se existe uma família $\left\{p_{\xi}: \xi<\kappa\right\}$ de ultrafiltros seletivos $\leq_{\mathrm{RK}}$-incomparáveis, então para cada $J \in \mathbb{Z}^{(k)} \backslash\{0\}$, existe um homomorfismo $\phi_{J}: \mathbb{Z}^{(\kappa)} \rightarrow \mathbb{T}$ satisfazendo

i. $\phi_{J}(J) \neq 0 \in \mathbb{T}$;

ii. para cada $0<\xi<\kappa, p_{\xi}-\lim _{n \in \omega} \phi_{J}\left(f_{\xi}(n)\right)=\phi_{J}\left(\chi_{\xi}\right)$.

Demonstração. Seja $J \in \mathbb{Z}^{(\kappa)} \backslash\{0\}$. De acordo com o lema 3.3.9, considerando a enumeração dada de $\mathscr{F}$, existe um subconjunto enumerável $E \in[\kappa]^{\omega}$ de maneira que para cada $\xi \in$ 
$E \backslash\{0\}, \bigcup_{n \in \omega} \operatorname{supp} f_{\xi}(n) \subseteq E$. Aplicando o lema 3.3.5, considerando o conjunto $E$ obtido, e posteriormente aplicando o lema 3.3.8, obtemos um homomorfismo $\phi: \mathbb{Z}^{(E)} \rightarrow \mathbb{T}$ satisfazendo

a. $\phi(J) \neq 0 \in \mathbb{T}$;

b. para cada $\xi \in E \backslash\{0\}, p_{\xi}-\lim _{n \in \omega} \phi\left(f_{\xi}(n)\right)=\phi\left(\chi_{\xi}\right)$.

Para cada $\xi \in \kappa$, existe em $\mathbb{Z}^{(\kappa)}$ uma cópia isomorfa do grupo $\mathbb{Z}^{(E \cup \xi)}$; abusando um pouco da notação, assumiremos que de fato, $\mathbb{Z}^{(E \cup \xi)}$ é subgrupo de $\mathbb{Z}^{(\kappa)}$ o que nos permite construir o isomorfismo $\phi_{J}$ como extensão do homomorfismo $\phi$ ao grupo $\mathbb{Z}^{(\kappa)}$.

Em primeiro lugar, para cada $H \in \mathbb{Z}^{(E)}$, definimos $\phi_{J}(H):=\phi(H)$. Pela propriedade b), $\phi_{J}\left(\chi_{\xi}\right)=\phi\left(\chi_{\xi}\right)=p_{\xi}-\lim _{n \in \omega} \phi\left(f_{\xi}(n)\right)$ para cada $\xi \in E \backslash\{0\}$.

Seja $\eta \in \kappa \backslash E$ o menor ordinal para o qual não temos definido $\phi_{J}\left(\chi_{\eta}\right)$. Se $\eta=0$, estende$\operatorname{mos} \phi_{J}$ a $\mathbb{Z}^{(E \cup\{0\})}$ arbitrariamente. Se $\eta>0$, observe que como $\bigcup_{n \in \omega} \operatorname{supp} f_{\eta}(n) \subseteq \eta$, para cada $n \in \omega$ temos $f_{\eta}(n) \in \mathbb{Z}^{(E \cup \eta)} \operatorname{logo}$, o elemento $\phi_{J}\left(f_{\eta}(n)\right)$ já está definido. Como $\mathbb{T}$ é compacto, é, em particular, $p_{\xi}$-compacto, logo se consideramos a seqüência $\left\{\phi_{J}\left(f_{\eta}(n)\right): n \in \omega\right\} \subseteq \mathbb{T}$, podemos definir

$$
\phi_{J}\left(\chi_{\eta}\right)=p_{\xi}-\lim _{n \in \omega} \phi_{J}\left(f_{\eta}(n)\right)
$$

Naturalmente, $\phi_{J}(J)=\phi(J) \neq 0$ e, de acordo com a propriedade b) e a definição anterior, $\phi_{J}$ satisfaz também ii), o que completa a prova.

Teorema 3.3.11. Seja $\kappa$ um cardinal satisfazendo $\kappa^{\omega}=\kappa \geq \mathfrak{c}$. Se existe uma família $\left\{p_{\xi}: \xi<\kappa\right\}$ de ultrafiltros seletivos $\leq_{\mathrm{RK}}$-incomparáveis, então existe uma topologia de grupo $\tau$ sobre $G=\mathrm{A}(\kappa)$ de modo que $(G, \tau)$ é um grupo van Douwen.

Demonstração. Preservando a notação exposta até aqui, seja $\left\{f_{\xi}: 0<\xi<\kappa\right\}$ uma enumeração de $\mathscr{F}$ de maneira que para cada $\xi<\kappa, \bigcup_{n \in \omega} \operatorname{supp} f_{\xi}(n) \subseteq \xi$.

Pelo lema 3.3.10, para cada $J \in \mathbb{Z}^{(\kappa)} \backslash\{0\}$, existe um homomorfismo $\phi_{J}: \mathbb{Z}^{(\kappa)} \rightarrow \mathbb{T}$ satisfazendo as condições i) e ii) referidas nesse lema. Seja $\Phi=\Delta\left\{\phi_{J}: J \in \mathbb{Z}^{(\kappa)} \backslash\{0\}\right\}$, isto é, para cada $f \in \mathbb{Z}^{(\kappa)}$ define-se

$$
\Phi(f)=\left\{\phi_{J}(f): J \in \mathbb{Z}^{(k)} \backslash\{0\}\right\}
$$

Como $\phi_{J}(J) \neq 0$ para cada $J \in \mathbb{Z}^{(\kappa)} \backslash\{0\}$, pelo lema 3.3 .3 obtemos que $\Phi: \mathbb{Z}^{(\kappa)} \rightarrow \mathbb{T}^{\mathbb{Z}^{(\kappa)} \backslash\{0\}}$ é um monomorfismo, logo $\Phi\left[\mathbb{Z}^{(\kappa)}\right]$ é isomorfo a $\mathbb{Z}^{(\kappa)}$. Para cada $\xi \in \kappa$, seja $x_{\xi}:=\Phi\left(\chi_{\xi}\right)$. Note-se que o conjunto $\left\{x_{\xi}: \xi \in \kappa\right\}$ constitui uma base do grupo abeliano livre $\Phi\left(\mathbb{Z}^{(\kappa)}\right)$. Seguindo esta 
notação, temos que para cada $\xi \in \kappa$ e $n \in \omega$,

$$
\begin{aligned}
\phi_{J}\left(f_{\xi}(n)\right) & =\phi_{J}\left(\sum_{\mu \in \operatorname{supp} f_{\xi}(n)} f_{\xi}(n)(\mu) \chi_{\mu}\right) \\
& =\sum_{\mu \in \operatorname{supp} f_{\xi}(n)} f_{\xi}(n)(\mu) \phi_{J}\left(\chi_{\mu}\right) \\
& =\sum_{\mu \in \operatorname{supp} f_{\xi}(n)} f_{\xi}(n)(\mu) x_{\mu} \\
& =x_{f_{\xi}(n)}
\end{aligned}
$$

Daí, a condição ii) que dá o resultado do lema 3.3.10 pode-se exprimir como

$$
p_{\xi}-\lim _{n \in \omega} x_{f_{\xi}(n)}=x_{\xi}
$$

De acordo com a construção, dados $\xi, \eta \in \kappa$ distintos temos que $x_{\xi} \neq x_{\eta}$. Observemos que para cada $0<\xi<\kappa, x_{\S}$ é, em particular, ponto de acumulação da seqüência $\left\{x_{f_{\xi}(n)}: n \in \omega\right\} \subseteq \mathbb{T}$. De acordo com o lema 3.3.4, o grupo abeliano livre $G=\left\langle x_{\xi}: 0<\xi<\kappa\right\rangle=\Phi\left[\mathbb{Z}^{(\kappa)}\right]$ é um grupo de van Douwen.

\subsubsection{Existência de ultrafiltros seletivos e MA: Alguns resultados de con- sistência}

Lembramos que o Axioma de Martin é a asserção de que para cada ordem parcial $\mathbb{P}$ satisfazendo c.c.c. se $\mathcal{D}$ é uma coleção de subconjuntos densos em $\mathbb{P}$ tal que $|\mathcal{D}|<2^{\aleph_{0}}$, existe um filtro $\mathbb{G} \subseteq \mathbb{P}$ intersetando cada elemento de $\mathcal{D}$.

Assim, podemos dizer que MA falha num modelo $\mathcal{M}$, se $\mathcal{M} \not \neq \mathrm{CH}$ e existem uma ordem parcial $\mathbb{P} \in \mathcal{M}$ satisfazendo a condição de cadeia enumerável e uma família $\mathcal{D}$ de subconjuntos $\mathbb{P}$-densos, onde $|\mathcal{D}|=\aleph_{1}$, de maneira tal que nenhum filtro em $\mathbb{P}$ é $\mathcal{D}$-genérico sobre $\mathbb{P}$.

Se MA não é satisfeito, uma maneira de medir o grau dessa falha é pelo número de ordens parciais satisfazendo a c.c.c. para as quais não existe nenhum filtro $\mathcal{D}$-genérico para cada coleção $\mathcal{D} \in[\mathbb{P}]^{<2^{\aleph_{0}}}$ formada por subconjuntos densos de $\mathbb{P}$. Em conseqüência, resulta natural a seguinte definição devida a J. E. Baumgartner:

Definição 3.3.12. O Axioma de Martin falha totalmente no sentido de Baumgartner num modelo $\mathcal{M}$ se $\mathcal{M} \not \mathrm{CH}$ e para qualquer ordem parcial atômica $\mathbb{P} \in \mathcal{M}$ existe uma família $\mathcal{D}$ de cardinalidade $\boldsymbol{\aleph}_{1}$ de conjuntos $\mathbb{P}$-densos, de maneira tal que nenhum filtro em $\mathbb{P}$ é $\mathcal{D}$-genérico.

Baumgartner, em [6] mostrou que é relativamente consistente com ZFC a falha total do 
Axioma de Martin:

Teorema 3.3.13. Sejam $\mathcal{M}$ é um modelo de ZFC+ $\left(2^{\aleph_{0}}=\boldsymbol{\aleph}_{1}\right)$ e $\kappa$ um cardinal em $\mathcal{M}$ satisfazendo $\kappa=\kappa^{\omega} \geq \omega_{2}$. Se $\mathbb{S}_{\omega}^{\kappa}$ representa a noção de forcing "side-by-side" de Sacks de comprimento $\kappa e$ $\mathbb{G}$ é um filtro $\mathbb{S}_{\omega}^{\kappa}$-genérico, então MA falha totalmente no sentido de Baumgartner na extensão $\mathcal{M}[\mathbb{G}]$.

De acordo com o exemplo 1.1.36, sob as condições do teorema anterior, temos que a noção de forcing $\mathbb{S}_{\omega}^{\kappa}$ preserva todos os cardinais e além disso, $(\mathfrak{c}=\kappa)^{\mathcal{M}[\mathbb{G}]}$.

No que se refere a ultrafiltros seletivos, em [7] encontra-se o seguinte resultado:

Teorema 3.3.14. Seja $M$ um modelo de ZFC+( $\left.2^{\aleph_{0}}=\boldsymbol{\aleph}_{1}\right)+\left(2^{\aleph_{1}}=\boldsymbol{\aleph}_{2}\right)$. Então, se $\mathbb{S}_{\mathrm{\omega}_{2}}$ representa a iteração de suporte enumerável do forcing de Sacks de comprimento $\omega_{2}$ e $\mathbb{G}$ é um filtro $\mathbb{S}_{\omega_{2}}$ genérico, então em $M[\mathbb{G}]$ cada ultrafiltro seletivo em $M[\mathbb{G}]$ é $\boldsymbol{\aleph}_{1}$-gerado e $2^{\aleph_{0}}=2^{\aleph_{1}}=\boldsymbol{\aleph}_{2}$. Assim, na extensão $M[\mathbb{G}]$ existem exatamente $2^{\aleph_{0}}$ ultrafiltros seletivos.

De acordo com o anterior, a existência de ultrafiltros seletivos é compatível com a noção de forcing $\mathbb{S}_{\omega_{2}}$ porém, usando dita ordem parcial, o tamanho do contínuo não pode ser maior do que $\mathfrak{\aleph}_{2}$. Por sua vez, considerando a noção de forcing $\mathbb{S}_{\omega}^{\kappa}$ o contínuo pode ser arbitrariamente grande e, de acordo com o seguinte resultado de Laver [49], no modelo extensão também existem ultrafiltros seletivos:

Teorema 3.3.15. Nas condições do teorema 3.3.13, considerando a noção de forcing side-by-side de Sacks $\mathbb{S}_{\omega}^{\kappa}$, os ultrafiltros seletivos no modelo $M$ formam uma base para os ultrafiltros seletivos na extensão $M[\mathbb{G}]$.

Como conseqüência dos teoremas 3.3.13 e 3.3.15, temos que é consistente a falha total de MA no sentido de Baumgartner com a existência de ultrafiltros seletivos.

Agora, no que concerne a existência de topologias enumeravelmente compactas sobre os grupos abelianos livres, podemos estabelecer os seguintes resultados:

Teorema 3.3.16. É consistente a falha total de MA no sentido de Baumgartner com a existência de uma topologia de grupo enumeravelmente compacta definida sobre o grupo abeliano livre $\mathrm{A}(\mathfrak{c})$.

Demonstração. Seja $\mathcal{M}$ um modelo de $\mathrm{GCH}$, e seja $\left(\kappa=\omega_{2}\right)^{\mathcal{M}}$. De acordo com Blass [12] em $\mathcal{M}$, existem $\kappa=2^{c}$ ultrafiltros seletivos. Pelo teorema 3.3.13, considerando a noção de forcing $\mathbb{S}_{\omega}^{\kappa}$, na extensão $\mathcal{M}[\mathbb{G}]$ temos que MA falha totalmente no sentido de Baumgartner e existe uma família de $(\kappa=\mathfrak{c})^{\mathcal{M}\left[{ }^{\mathcal{G}]}\right.}$ ultrafiltros seletivos $\leq_{\mathrm{RK}}$-incomparáveis. De acordo com 
o teorema 3.3.11, em $\mathcal{M}[\mathbb{G}]$, existe uma topologia de grupo enumeravelmente compacta no grupo abeliano livre gerado por $\kappa=\mathfrak{c}$ elementos.

Teorema 3.3.17. É consistente a existência de uma topologia de grupo enumeravelmente compacta sobre $\mathrm{A}\left(2^{\mathrm{c}}\right)$ com a falha total de MA no sentido de Baumgartner.

Demonstração. Seja $\mathcal{M}$ um modelo de $\mathrm{GCH}$ e sejam $\kappa$ e $\lambda$ cardinais regulares não enumeráveis tais que $\kappa<\lambda$. Mediante o uso da noção de forcing de Easton (1.1.33), podemos forçar $2^{\omega_{1}}=\lambda$, obtendo um modelo $\mathcal{N}$ no qual $\mathrm{CH}$ é satisfeito, existem $2^{\omega_{1}}$ ultrafiltros seletivos, $2^{\omega_{1}}=2^{\kappa}=\lambda$, $\kappa^{\omega}=\kappa$ e $\kappa<\lambda$ são cardinais não enumeráveis. Forçando sobre $\mathcal{N}$ com a noção de forcing side-by-side de Sacks $\mathbb{S}_{\omega}^{\kappa}$, em vista dos teoremas 3.3.13 e 3.3.15, temos que MA falha totalmente no sentido de Baumgartner, $\kappa=\mathfrak{c}$ e existem $\lambda$ ultrafiltros seletivos no modelo extensão $\mathcal{N}[\mathbb{G}]$. Ainda mais, como $\left(\left|\mathbb{S}_{\omega}^{\kappa}\right|=\kappa\right)^{\mathcal{N}[G]}$ e $\mathbb{S}_{\omega}^{\kappa}$ é $\omega_{2}$-c.c., existem no máximo $\left(\kappa^{\omega_{1}}=\lambda\right)^{\mathcal{N}[G]} \mathbb{S}_{\omega}^{\mathcal{K}}$-bons nomes. Assim, $\left(2^{\mathfrak{C}}=2^{\mathcal{K}}=\lambda\right)^{\mathcal{N}[G]}$. Em virtude do teorema 3.3.11, existe uma topologia de grupo enumeravelmente compacta sobre $A\left(2^{\mathfrak{c}}\right)$ no modelo $\mathcal{N}[\mathbb{G}]$. 


\section{CAPÍTULO 4}

\section{Invariantes cardinais em grupos enumeravelmente compactos}

A maior parte das questões envolvendo propriedades próximas à compacidade, tem surgido com o intuito de fazer mais gerais os resultados que de fato são obtidos quando se trata de espaços compactos, como tem-se observado ao longo do texto. O estudo de invariantes cardinais, é um assunto que interessa particularmente, dada a grande quantidade de resultados a este respeito envolvendo espaços compactos. Um dos resultados envolvendo o peso e a cardinalidade de um espaço compacto, foi estabelecido no teorema 1.2.19: para qualquer espaço compacto temos que $w(X) \leq|X|$. Motivado nesse resultado, Hodel em [42] formulou a seguinte questão:

Questão 4.1. Seja $\kappa>\omega$ um cardinal. Existe um espaço regular e enumeravelmente compacto $X$ tal que $|X|=\kappa$ e $w(X)>\kappa ?$

Após de formular essa questão, Hodel refere que Comfort mostrou que a resposta à questão é afirmativa para cardinais $\kappa$ satisfazendo $\kappa=\kappa^{\omega}$. O trabalho de van Douwen [27], mostra que a resposta à pergunta de Hodel é negativa para certos outros $\kappa$, porém que podem existir grupos pseudocompactos de cardinalidade $\kappa$ tal que $\kappa^{\omega} \neq \kappa$. De fato, em [27] é estudada a questão mais geral:

Questão 4.2. Para quais cardinais $\kappa$ e $\lambda$ existe um espaço pseudocompacto (pseudocompacto $e$ homogêneo) X tal que $|X|=\kappa e w(X)=\lambda$ ?

Neste capítulo, iremos estudar as relações que devem satisfazer o peso e a cardinalidade de um grupo pseudocompacto e enumeravelmente compacto de acordo com os trabalhos de E. van Douwen [27] e A. H. Tomita [67]. 


\subsection{Peso e cardinalidade de grupos pseudocompactos}

Como é natural, as relações entre as funções cardinais são ainda mais fortes considerando grupos topológicos do que espaços topológicos compactos sem envolver uma estrutura algébrica compatível. De fato, mencionamos anteriormente o seguinte resultado estabelecido de maneira independente por Ivanovskiǐ (1958) e Kuz'minov (1959):

Teorema 4.1.1. Todo grupo compacto $G$ é imagem contínua do espaço $2^{w(G)}$.

Produto do teorema 4.1.1, das relações entre os invariantes cardinais em espaços compactos e do teorema de Hewitt-Marczewski-Pondiczery, o resultado a seguir proporciona de maneira eficaz uma caraterização do peso e da cardinalidade de um grupo compacto infinito:

Teorema 4.1.2. Seja G um grupo topológico infinito compacto. Então:

a) $\chi(G)=w(G)$

b) $|G|=2^{w(G)}$;

c) $d(G)=\ln w(G)$ onde $\ln (\kappa)=\min \left\{\lambda: \lambda\right.$ é cardinal e $\left.2^{\lambda} \geq \kappa\right\}$.

Demonstração. a) Como $G$ é um espaço homogêneo e assumimos que não é discreto, temos que $\chi(p, G)=\chi(G)=\kappa$ para todo $p \in G$. Naturalmente, $\chi(G) \leq w(G)$. Agora, seja $\mathscr{H}$ uma base local na identidade $e_{G}$ de cardinalidade $\kappa$. Como $G$ é compacto, para cada $U \in \mathscr{H}$ existe um subconjunto finito $S_{U} \subseteq G$ tal que $S_{U} U=G$. Para cada $U \in \mathscr{H}$, seja $\mathscr{B}_{U}=\left\{x U: x \in S_{U}\right\}$. Note-se que a família $\mathscr{B}=\bigcup\left\{\mathscr{B}_{U}: U \in \mathscr{H}\right\}$ constitui uma base para $G$ e satisfaz $|\mathscr{B}| \leq \kappa$. Daí, $w(G) \leq \kappa=\chi(G)$.

b) Como vimos no item a), para cada ponto $p \in G$, temos $\chi(p, G)=\chi(G)=w(G)$. Assim, o teorema de Čech-Pospíšil (1.2.20) implica que $|G| \geq 2^{w(G)}$. Como todo espaço $T_{1}$ satisfaz $|X| \leq 2^{w(G)}$, concluímos que $|G|=2^{w(G)}$.

c) Pelo teorema 4.1.1, $G$ é diádico, logo para algum cardinal $\kappa$ existe uma função contínua e sobrejetora $f:\{0,1\}^{\kappa} \rightarrow G$. Pode-se assumir que $\kappa=w(G)$. Pelo teorema 1.2.17, temos que $d\left(\{0,1\}^{\kappa}\right)=\ln \kappa, \operatorname{logo} d(G) \leq d\left(2^{w(G)}\right)=\ln w(G)$. Em virtude da proposição 1.2.16, $w(G) \leq 2^{d(G)} ;$ portanto, $\ln w(G) \leq d(G)$. 
Note-se que, pelo lema de Zermelo-König, em particular $w(G)<|G|$ para qualquer grupo compacto infinito. No entanto, se $H=\left\{x \in \mathbb{T}^{\mathrm{c}}:|\operatorname{supp} x| \leq \omega\right\} \subseteq \mathbb{T}^{\mathrm{c}}$ e $\tau$ a topologia como subespaço de $\mathbb{T}^{\mathrm{c}}$ definida em $H$ não é difícil ver que $(H, \tau)$ é enumeravelmente compacto e $|H|=w(H)=\mathfrak{c}$. De fato, pode-se mostrar que ainda, a cardinalidade de um grupo pseudocompacto pode ser menor do que o peso do grupo.

Antes de prosseguir, convém lembrar uma caracterização mais dos espaços pseudocompactos.

Proposição 4.1.3. Seja X um espaço de Tychonoff. As seguintes afirmações são equivalentes:
i. X é pseudocompacto;
ii. toda família localmente finita de subconjuntos abertos não vazios de X é finita;

iii. $X$ é $G_{\delta}$-denso em cada uma das suas compactificações;

iv. $X$ é $G_{\delta}$-denso em $\beta X$.

Demonstração. i) $\Rightarrow$ ii): Seja $X$ pseudocompacto e suponhamos por absurdo que existe uma família infinita $\mathcal{U}=\left\{U_{n}: n \in \omega\right\}$ de abertos não vazios de $X$ localmente finita. Para cada $n \in \omega$, seja $x_{n} \in U_{n}$ e tomemos $f_{n}: X \rightarrow \mathbb{R}^{\geq 0}$ contínua tal que $f_{n}\left(x_{n}\right)=n$ e $f_{n}(x)=0$ para todo $x \in X \neq U_{n}$. Definimos $f=\sum_{n<\omega} f_{n}$. Como $\mathcal{U}$ é localmente finita, para cada $x \in X$ existe uma vizinhança aberta $V$ de $x$ que interseta um número finito de elementos de $\mathcal{U}$; portanto, em $V$, $f$ coincide com a soma de um número finito de funções contínuas, sendo também contínua no aberto $V$. Sendo esta a situação para qualquer elemento $x$ do espaço, segue que $f$ é contínua em $X$, mas $f$ não é limitada, uma contradição.

i) $\Rightarrow$ iii): Seja $B(X)$ uma compactificação de $X$. Suponhamos por absurdo que existe uma coleção de abertos não vazios em $B(X),\left\{U_{n}: n \in \omega\right\}$, tal que $p \in \bigcup_{n \in \omega} U_{n} \subseteq B(X) \backslash X$. Notemos que isto é equivalente a dizer que $X$ não interseta todos os conjuntos $G_{\delta} \operatorname{em~} B(X)$. Tomemos, para cada $n \in \omega$, uma função contínua $f_{n}: B(X) \rightarrow[0,1]$ tal que $f_{n}(p)=0$ e $f_{n}(x)=1$ para cada $x \in B(X) \backslash U_{n}$. Seja $f=\sum_{n \in \omega} \frac{f_{n}}{2^{n}}$. Consideremos $f=f \uparrow_{X}$; como $f$ é contínua em $B(X)$ e $f(p)=0$, segue que $\frac{1}{g}$ é contínua mas não é limitada em $X$, uma contradição.

ii) $\Rightarrow$ i): Suponhamos que existe uma função contínua e não limitada $f: X \rightarrow \mathbb{R}$. Seja $x_{0} \in X$ e, recursivamente, para cada $n \in \omega$, escolhemos $x_{n+1} \in X$ tal que $f\left(x_{n+1}\right)>f\left(x_{n}\right)+1$. Como $f$ é contínua, para cada $n \in \omega$ o conjunto $U_{n}=f^{-1}\left[\left(f\left(x_{n}\right)-1, f\left(x_{n}\right)+1\right)\right]$ é aberto no espaço $X$. De acordo com a nossa escolha, temos que $\left\{U_{n}: n \in \omega\right\}$ é uma família infinita de abertos não vazios em $X$ localmente finita, uma contradição. 
iii) $\Rightarrow$ iv): $\quad$ É obvio.

iv) $\Rightarrow$ i): Suponhamos por absurdo que existe uma função $f: X \rightarrow \mathbb{R}$ contínua e não limitada, de modo que $f(x) \leq 1$ para todo $x \in X$. Seja $\beta g$ a extensão de Čech-Stone da função contínua $g=\frac{1}{f}$. Como inf $g=0,(\beta g)^{-1}[\{0\}] \neq \varnothing ;$ observe-se também que $(\beta g)^{-1}[\{0\}]$ é fechado e portanto, $G_{\delta}$ em $\beta X$ mas, $(\beta g)^{-1}[\{0\}] \cap X=\varnothing$ o que contradiz iv).

A primeira das restrições é estabelecida no seguinte resultado simples:

Proposição 4.1.4. Se X um espaço topológico pseudocompacto então

a. Se X não tem pontos isolados, então $|X| \geq \mathfrak{c}$;

b. Se X é infinito e homogêneo, então $X$ não tem pontos isolados.

Demonstração. a. A prova segue um argumento similar ao do teorema de Čech-Pospíšil, mediante a construção de uma árvore de Cantor. De maneira indutiva, para cada $n \in \omega$ construiremos uma coleção $\left\{V_{f}: f \in 2^{n}\right\}$ de subconjuntos abertos não vazios de $X$ tais que

i. Se $f \in 2^{n}$ e $m<n$, então $V_{f} \subseteq V_{f \vdash m}$;

ii. se $f, g \in 2^{n}$ e $f \neq g$, então $\overline{V_{f}} \cap \overline{V_{g}}=\varnothing$.

Se $n=0,2^{0}=\{\varnothing\}$ e tomamos $V_{\varnothing}=X$. Seja $n \in \omega$. Suponhamos que, para cada $m \leq n$, temos definido a coleção $\left\{V_{f}: f \in 2^{m}\right\}$ satisfazendo as condições i) e ii). Se $g \in 2^{n}$, definimos $f_{0}, f_{1} \in 2^{n+1}$ como $f_{0}=g^{-} 0$ e $f_{1}=g^{\frown} 1$. Como $V_{g} \neq \varnothing$ e $X$ é um espaço de Tychonoff sem pontos isolados, existem abertos não vazios $V_{f_{0}}$ e $V_{f_{1}}$ tais que $\overline{V_{f_{0}}} \cup \overline{V_{f_{1}}} \subseteq V_{g}$ e $\overline{V_{f_{0}}} \cap \overline{V_{f_{1}}}=\varnothing$. Assim, $V_{f_{0}}$ e $V_{f_{1}}$ satisfazem as condições i)-ii).

Por indução, para cada $n \in \omega$ obtemos uma coleção $\left\{V_{g}: g \in 2^{n}\right\}$ de subconjuntos abertos em $X$ satisfazendo as condições i) e ii). Para cada $f \in 2^{\omega}$, seja

$$
W_{f}=\bigcap_{n<\omega} \overline{V_{f \eta_{n}}}
$$

Pela condição i), para cada $f \in 2^{\omega},\left\{V_{f \uparrow n}: n \in \omega\right\}$ é uma cadeia decrescente de subconjuntos abertos não vazios em $X$. Como $X$ é pseudocompacto e de Tychonoff, pelo item ii) da proposição 1.2.9 temos que existe $x_{f} \in W_{f}=\bigcap_{n<\omega} \overline{V_{f{ }_{n}}}$.

Pela condição ii), se $f, h \in 2^{\omega}$ são funções distintas, então $\overline{V_{f}} \cap \overline{V_{h}}=\varnothing$, de modo que $x_{f} \neq x_{h}$. Como $\left\{x_{f}: f \in 2^{\omega}\right\} \subseteq X,|X| \geq \mathfrak{c}$. 
b. Se $X$ tivesse um ponto isolado, $X$ seria um espaço discreto pois ele é homogêneo. Então, como $\bigcup_{x \in X}\{x\}$ é uma cobertura aberta de $X$, pela proposição 1.2.9 item iv) existe $F \in[X]^{<\omega}$ tal que $X=\bigcup_{x \in F} \overline{\{x\}}=F$, ou seja $X$ é finito, uma contradição. Daí, $X$ não possui pontos isolados.

De acordo com os resultados da proposição 1.2.16, temos que para um espaço topológico $X$, deve-se satisfazer $w(X) \leq 2^{|X|}$ e $|X| \leq 2^{w(X)}$. Se tratando de um espaço pseudocompacto, é obtida uma condição adicional envolvendo a cofinalidade do cardinal do espaço.

Teorema 4.1.5. Seja $X$ um espaço tal que $\operatorname{cf}(|X|)=\omega$. As seguintes afirmações são válidas:

i. Se X é pseudocompacto, então $w(X) \leq 2^{<|X|}$;

ii. se $X$ é pseudocompacto e homogêneo, então $d(X)<|X|$, logo $w(X) \leq 2^{\mu}$ para algum $\mu<|X|$.

Demonstração. Antes de tudo, como $\operatorname{cf}(|X|)=\omega$ podemos fixar uma coleção enumerável $\mathscr{A}$ de subconjuntos de $X$ tal que $X=\bigcup \mathscr{A}$ e $|A|<|X|$ para cada $A \in \mathscr{A}$. Também, é possível fixar um subconjunto enumerável $M$ de cardinais tal que $\sup (M)=|X|$ e $\mu<|X|$ para cada $\mu \in M$.

i. Observemos que se existir $x \in \beta X \backslash \bigcup_{A \in \mathscr{A}} \bar{A}^{\beta X}$, para cada $A \in \mathscr{A}$ podemos tomar um conjunto $U_{A}$ aberto em $\beta X$ tal que $x \in U_{A}$ e $U_{A} \cap A=\varnothing$; como $|\mathscr{A}|=\omega$ então $x \in W=\bigcap_{A \in \mathscr{A}} U_{A}$ é um $G_{\delta}$ no espaço $\beta X$ satisfazendo

$$
\begin{aligned}
W \cap X & =W \cap \bigcup_{A \in \mathscr{A}} A \\
& =\bigcup_{A \in \mathscr{A}} W \cap A \\
& \subseteq \bigcup_{A \in \mathscr{A}} U_{A} \cap A=\varnothing
\end{aligned}
$$

Em virtude da proposição 4.1.3, $X$ não é pseudocompacto, uma contradição. Portanto, $\beta X=\bigcup_{A \in \mathscr{A}} \bar{A}^{\beta X}$.

Para cada $A \in \mathscr{A}$ temos que $w\left(\bar{A}^{\beta X}\right) \leq 2^{d\left(\bar{A}^{\beta X}\right)} \leq 2^{|A|}$, logo, o espaço compacto e infinito $\beta X$ é reunião de uma quantidade enumerável de subespaços de peso no máximo $2^{<|X|}=$ $\sup \left\{2^{\kappa}: \kappa<|X|\right\}$ e, consequentemente, $w(\beta X) \leq 2^{<|X|} \omega=2^{<|X|}$. Como uma base do espaço $X$ pode ser obtida a partir de uma base de $\beta X$, segue que $w(X) \leq w(\beta X) \leq 2^{<|X|}$. 
ii. Pela proposição 4.1.3, temos que $X$ é denso em $\beta X$ constituindo desse modo um espaço de Baire; da anterior observação e da igualdade $X=\bigcup \mathscr{A}$, decorre que existe $A \in \mathscr{A}$ tal que $\operatorname{int}\left(\bar{A}^{X}\right)_{X} \neq \varnothing$. Dado que $d\left(\operatorname{int}\left(\bar{A}^{X}\right)_{X}\right) \leq|A|<|X|$ e $X$ é homogêneo, para cada $x \in X$, existe uma vizinhança aberta $x \in U_{x}$ satisfazendo $d\left(U_{x}\right)<|X|$.

Para cada $A \in \mathscr{A}$ e $\mu \in M$, definimos

$$
V_{A, \mu}=\bigcup\left\{U_{x}: x \in A, d\left(U_{x}\right)<\mu\right\}
$$

Se $\mathcal{V}=\left\{V_{A, \mu}: A \in \mathscr{A}, \mu \in M\right\}$, temos que $\mathcal{V}$ é uma cobertura aberta enumerável de $X$, pois $\mathscr{A}$ é uma cobertura de $X$ e $M$ é cofinal em $|X|$. Notemos que para cada $A \in \mathscr{A}, \mu \in M$, $d\left(V_{A, \mu}\right) \leq|A| \mu<|X|$.

De acordo com a proposição 1.2.9 item iv), existe $F \in[\mathcal{V}]^{<\omega}$ tal que $X=\bigcup_{V \in F} \bar{V}^{X}$, logo, $d\left(\bigcup_{V \in F} V\right) \leq|X|$. Como $X=\bigcup_{V \in F} \bar{V}^{X} \subseteq \bar{\bigcup}_{V \in F} V$, $\bigcup_{V \in F} V$ é denso em $X$, de modo que $d(X) \leq$ $d\left(\bigcup_{V \in F} V\right) \leq|X|$

Corolário 4.1.6. Se $X$ é pseudocompacto e $|X|$ é um cardinal fortemente limite satisfazendo $\operatorname{cf}(|X|)=\omega$, então alguns mas não todos os pontos de $X$ tem uma vizinhança de cardinalidade estritamente menor que $|X|$.

Demonstração. De acordo com a prova do item ii) do teorema anterior, como $X$ é pseudocompacto (e assim um espaço de Baire), temos que existe $\breve{A} \in \mathscr{A}$ tal que $\breve{U}:=\operatorname{int}(\overline{\breve{A}}) \neq \varnothing$; notemos então, que $\breve{U}$ é um aberto não vazio de $X$ para o qual $d(\breve{U}) \leq|\breve{U}| \leq|\breve{A}|<|X|$, logo, pela proposição 1.2.16, segue que $|\breve{U}| \leq 2^{2^{d(\breve{U})}}<|X|$ pois $|X|$ é fortemente limite.

Se isso acontecer para todos os pontos de $X$, obteríamos uma cobertura aberta enumerável de $X$, digamos, $\mathcal{V}$ tal que para cada $U \in \mathcal{V},|U|<|X|$. Pela proposição 1.2.9 item iv), existe $F \in[\mathcal{V}]^{<\omega}$ tal que $X=\bigcup_{V \in F} \bar{V}=\overline{\bigcup_{V \in F} V}$, mas daí, $d(X) \leq|X|$. Pela proposição 1.2.16, $|X| \leq 2^{2^{d(X)}}$, isto é $|X|<|X|$, uma contradição.

Corolário 4.1.7. Se X é um espaço de Baire homogêneo tal que $\operatorname{cf}(|X|)=\omega$, então $w(X) \leq 2^{\mathfrak{m}}|X|$ para algum $\mathrm{m}<|X|$.

Demonstração. Como $X$ é de Baire, de acordo com a prova do item ii) do teorema 4.1.5, temos

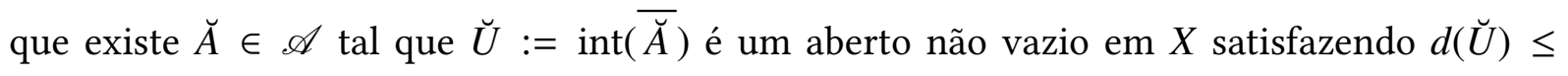
$|\breve{U}| \leq|A|=\mathfrak{m}<|X|$. Dado que $X$ é homogêneo, cada $x \in X$ possui uma vizinhança aberta $U_{x}$ satisfazendo $d\left(U_{x}\right) \leq \mathrm{m}<|X|$; assim, em virtude da proposição 1.2.16 $w\left(U_{x}\right) \leq 2^{d\left(U_{x}\right)} \leq 2^{\mathfrak{m}}$. 
Para cada $A \in \mathscr{A}$ seja $V_{A}=\bigcup_{x \in A} U_{x}$, de modo que $\mathcal{V}=\left\{V_{A}: A \in \mathscr{A}\right\}$ é uma cobertura aberta enumerável de $X$. Como $w\left(V_{A}\right) \leq|A| 2^{\mathfrak{m}}$ para cada $A \in \mathscr{A}$ temos que

$$
w(X) \leq \sum_{A \in \mathscr{A}}|A| 2^{\mathfrak{m}} \leq 2^{\mathfrak{m}} \sum_{A \in \mathscr{A}}|X|=2^{\mathfrak{m}}|X|
$$

Corolário 4.1.8. Se $X$ é pseudocompacto e $|X|$ é fortemente limite tal que $\operatorname{cf}(|X|)=\omega$, então $w(X)=|X|$ e $X$ não é homogêneo.

Demonstração. Pelo item i) do teorema 4.1.5 temos que $w(X) \leq 2^{<|X|} \leq|X|$; se $w(X)<|X|$, a proposição 1.2.16 implica $|X| \leq 2^{w(X)}<|X|$, uma contradição. Daí, $w(X)=|X|$.

Se $X$ fosse homogêneo, pelo item ii) do teorema 4.1.5, $w(X) \leq 2^{\mu}$ para algum $\mu<|X|$, mas, como $|X|$ é fortemente limite $|X|=w(X)<|X|$, absurdo.

Corolário 4.1.9. Sob $\mathrm{GCH}$, se $X$ é um espaço pseudocompacto tal que $\operatorname{cf}(|X|)=\omega$, então $w(X)=$ $|X|$.

Demonstração. Pelo item i) do teorema 4.1.5, $w(X) \leq 2^{<|X|}$. Ao assumirmos GCH, obtém-se $w(X) \leq 2^{<|X|}=|X|$; se $w(X)<|X|$, então $|X| \leq 2^{w(X)}=w(X)^{+}$, logo $|X|=w(X)^{+}$o que contradiz $\operatorname{cf}(|X|)=\omega$, logo necessariamente $w(X)=|X|$.

Conclui-se que os resultados obtidos por van Douwen estabelecem que para quaisquer $\kappa$ e $\lambda$ satisfazendo $\lambda \leq 2^{\kappa}$ e $\kappa \leq 2^{\lambda}$ existe um espaço (grupo) $X$ tal que $|X|=\kappa$ e $w(X)=$ $\lambda$. Sob ZFC, não existem restrições adicionais sobre o peso e a cardinalidade de um espaço pseudocompacto (ainda mais, enumeravelmente compacto) sem pontos isolados, ou sobre o peso e a cardinalidade de um espaço pseudocompacto homogêneo (mesmo se fosse um grupo).

Teorema 4.1.10. Assumamos que a seguinte afirmação é verdadeira:

$$
\text { Se } \mu \geq \mathfrak{c} e \operatorname{cf}(\mu) \neq \omega \text {, então } \mu^{\omega}=\mu
$$

Sejam $\kappa$ e $\lambda$ cardinais que satisfazem $\mathrm{c} \leq \kappa \leq 2^{\lambda} e \lambda \leq 2^{\kappa}$. Então,

i. Se existe $\mu<\kappa$ de maneira que $\lambda \leq 2^{\mu}$ e $\mathrm{cf}(\kappa)=\omega$, então existe um grupo pseudocompacto $X$ tal que $|X|=\kappa e w(X)=\lambda$.

ii. Se adicionalmente, $\lambda \leq 2^{<\kappa}$ e $\mathrm{cf}(\kappa)=\omega$, então existe um espaço enumeravelmente compacto $X$ sem pontos isolados tal que $w(X)=\lambda e|X|=\mu$. 
Demonstração. Antes de começar com a prova convém mencionar que a afirmação @ é equivalente à hipótese dos cardinais singulares $(\mathrm{SCH})$, uma asserção consistente com ZFC. Consideremos o grupo compacto $L=2^{\lambda}$. Observemos que se $D \subseteq L$ é denso e $x \in D$, então $\chi(x, D)=\chi(x, L)=\lambda$. Como $\chi(D) \leq w(D) \leq w(L)=\lambda$ e $\lambda=\chi(x, D) \leq \chi(D)$, segue que $w(D)=\lambda$. Dado que os espaços a serem construídos são densos em $L$, segue que cada um deles tem peso $\lambda$.

Pela hipótese, $\lambda \leq 2^{\kappa}$. Assim, de acordo com o teorema 1.2.17 segue que $d(L) \leq \kappa$. Como ainda $\kappa \leq 2^{\lambda}$, temos que existe um subconjunto denso $F \subseteq L$ de cardinalidade $\kappa$ obtendo assim um subgrupo denso $(G=\langle F\rangle)$ de cardinalidade $\kappa$. A afirmação anterior implica que $w(X) \leq 2^{|X|}$ e $|X| \leq 2^{w(X)}$ são as únicas restrições envolvendo esses invariantes cardinais ainda quando os espaços são grupos.

Para mostrarmos o item i), suponhamos adicionalmente que $\operatorname{cf}(\kappa)=\omega$. Vamos construir por indução transfinita, uma família $\subseteq$-crescente de subgrupos $\left\{G_{\alpha}: \alpha<\omega_{1}\right\}$ tais que para cada $\alpha<\omega_{1}, G \subseteq G_{\alpha},\left|G_{\alpha}\right|=\kappa$ e cada subconjunto enumerável em $G_{\alpha}$ tenha um ponto de acumulação em $G_{\alpha+1}$. Este método, conhecido na literatura como clossing-off usado por V. Saks e R. M. Stephenson [56] deve-se originalmente a Frolík [32].

Seja $G_{0}=G$ e suponhamos que para $\gamma<\omega_{1}$, a família $\left\{G_{\alpha}: \alpha<\gamma\right\}$ tem sido definida satisfazendo

a. se $\alpha<\beta<\gamma$, então $G_{\alpha} \subseteq G_{\beta}$;

b. para cada $\alpha<\gamma$ tal que $\alpha+1 \leq \gamma$, toda seqüência em $G_{\alpha}$ tem um ponto de acumulação em $G_{\alpha+1}$;

c. $\left|G_{\alpha}\right|=\kappa$;

d. se $\alpha \leq \gamma$ é um ordinal limite, então $G_{\alpha}=\bigcup_{\beta<\alpha} G_{\alpha}$.

De acordo com a hipótese indutiva, basta supor que $\gamma=\alpha+1$. Como $G_{\alpha}$ é denso em $L$ e $\left|G_{\alpha}\right|=\kappa \leq 2^{\lambda}$, para cada seqüência em $G_{\alpha}$ podemos tomar um ponto de acumulação em $L$; daí, definimos $G_{\alpha+1}=\left\langle G_{\alpha} \cup\left\{x_{F} \in L: x_{F} \in F^{\prime}, F \in\left[G_{\alpha}\right]^{\omega}\right\}\right\rangle$. Observemos que a relação $\kappa^{\omega}=\kappa=\left|G_{\alpha}\right|$ implica $\left|G_{\alpha+1}\right|=\kappa$.

Por indução, a coleção $\left\{G_{\alpha}: \alpha<\omega_{1}\right\}$ satisfaz os nossos requerimentos. Se $X=\bigcup_{\alpha<\omega_{1}} G_{\alpha}$, naturalmente $X$ é um subgrupo denso de $L$, e, como $\left|G_{\alpha}\right|=\kappa$ para cada $\alpha<\omega_{1}$ e $\omega_{1} \leq \mathfrak{c} \leq \kappa$, $|X|=\kappa$. 
Dada qualquer seqüência $\left\{x_{n}: n \in \omega\right\} \subseteq X$, existe $\alpha<\omega_{1}$ tal que $\left\{x_{n}: n \in \omega\right\} \subseteq G_{\alpha}$; de fato, tomemos $f(0)=\min \left\{\beta<\omega_{1}: x_{0} \in G_{\beta}\right\}$ e para cada $n \geq 1$ definamos

$$
f(n)=\max \left\{\min \left\{\beta<\omega_{1}: x_{n} \in G_{\beta}\right\}, f(n-1)\right\}+1
$$

Como $\operatorname{cf}\left(\omega_{1}\right) \neq \omega$ e a função $f: \omega \rightarrow \omega_{1}$ é estritamente crescente, decorre que $\alpha=\sup \{f(n)$ : $n \in \omega\}<\omega_{1}$; portanto, a seqüência $\left\{x_{n}: n \in \omega\right\} \subseteq G_{\alpha}$ e possui um ponto de acumulação em $G_{\alpha+1} \subseteq X$. Acontecendo isso para cada seqüência em $X$, obtemos que $X$ é enumeravelmente compacto.

Suponhamos agora que $\kappa^{\omega} \neq \kappa>\mathfrak{c}$, e que existe $\mu<\kappa$ tal que $\lambda \leq 2^{\mu}$. Como $\mu^{+} \geq \mathfrak{c}$ e $\operatorname{cf}\left(\mu^{+}\right) \neq \omega$, por $(\$)$ temos que $\left(\mu^{+}\right)^{\omega}=\mu^{+}$, logo, podemos supor que $\mu^{\omega}=\mu$. De acordo com os argumentos acima, dado que $\lambda \leq 2^{\mu}$ e $\mu<2^{\lambda}$, L possui um subgrupo $H$, denso e enumeravelmente compacto tal que $|H|=\mu$. Como $\mu<\kappa \leq 2^{\lambda}$, podemos tomar um subconjunto $A:=\left\{x_{\alpha}: \alpha<\kappa\right\} \subseteq L \backslash H$; daí, o subgrupo $G=\langle A \cup H\rangle \subseteq L$ possui cardinalidade $\kappa$. Se $f: G \rightarrow \mathbb{R}$ é uma função contínua, como $H$ é enumeravelmente compacto (em particular pseudocompacto), existe $r>0$ tal que $f(H) \subseteq[-r, r] \subseteq \mathbb{R}$; daí, $f(G)=f\left(\bar{H}^{G}\right) \subseteq \overline{f(H)}^{\mathbb{R}} \subseteq$ $[-r, r]$, ou seja, $G$ é pseudocompacto.

Agora, suponhamos que $\kappa^{\omega} \neq \kappa>c$ e $\lambda \leq 2^{<\kappa}$ mas não existe $\mu<\kappa$ tal que $\lambda \leq 2^{\mu}$; notese que a última condição implica $\lambda=2^{<\kappa}$. Dado que $\kappa>\mathfrak{c}$ e $\kappa^{\omega} \neq \kappa$, ao estarmos assumindo (ه) segue que $\operatorname{cf}(\kappa)=\omega$, portanto, existe uma seqüência de cardinais $\mathcal{M}=\left\{\mu_{n}<\kappa: n \in \omega\right\}$ tal que $\kappa=\sup (\mathcal{M})$. Podemos assumir, sem perda de generalidade que para cada $n \in \omega, \mu_{n} \geq \mathfrak{c}$, e que $\mu_{n}$ é um cardinal sucessor, de onde $\operatorname{cf}\left(\mu_{n}\right)=\mu_{n} \neq \omega$, obtendo assim, pela afirmação (ه), que também $\mu_{n}^{\omega}=\mu_{n}$.

Para cada $n \in \omega$, considerando o cardinal $\mu_{n}=\mu_{n}^{\omega}$, usando um argumento de clausura (ou clossing-off) como o descrito inicialmente, podemos construir um grupo $G_{n}$ enumeravelmente compacto satisfazendo $\left|G_{n}\right|=\mu_{n}$ e $w\left(G_{n}\right)=2^{\mu_{n}}$. Seja $X=\bigsqcup_{n \in \omega} G_{n}$ a união disjunta da família de grupos $\left\{G_{n}: n \in \omega\right\}$; em $X$, seja $\varsigma$ a topologia inerente à soma topológica da família $\left\{G_{n}: n \in \omega\right\}$. Pela proposição 1.2.12 $(X, \varsigma)$ é enumeravelmente compacto; agora, seja $\tau=\varsigma \cup$ $\left\{U \subseteq X:\left|\left\{n \in \omega: G_{n} \nsubseteq U\right\}\right|<\omega\right\}$. É fácil ver que $\tau$ é uma topologia para $X$. Observemos que $(X, \tau)$ também é enumeravelmente compacto e, além disso, sem pontos isolados. Obviamente, $|X|=\kappa$ e $w(X)=2^{<\kappa}=\lambda$.

Convém ilustrar mediante dois exemplos que impõem condições distintas sobre a natureza dos cardinais a serem analisados, as restrições sobre invariantes cardinais que até agora achamos. 
Exemplo 4.1.11. Suponhamos que $\kappa$ é um cardinal fortemente limite de cofinalidade enumerável. A seguinte tabela sintetiza as condições necessárias e suficientes para a existência de um espaço topológico $X$ de cardinalidade $\kappa$ e peso $\lambda$ satisfazendo certas propriedades:

\begin{tabular}{|ll|}
\hline Estrutura de $X$ & Condição necessária e suficiente sobre $\lambda$ \\
\hline \hline Espaço topológico & $\kappa \leq \lambda \leq 2^{\kappa}$ \\
Grupo topológico & $\kappa \leq \lambda \leq 2^{\kappa}$ \\
Espaço enumeravelmente com- & $\lambda=\kappa$ \\
pacto ou pseudocompacto & $\begin{array}{l}\text { Para nenhum } \lambda \text { : pelo corolário 4.1.8 não exis- } \\
\text { tem espaços pseudocompactos homogêneos } \\
\text { Grupo pseudocompacto }\end{array}$ \\
& cuja cardinalidade seja fortemente limite. \\
\hline
\end{tabular}

Exemplo 4.1.12. De acordo com a noção de forcing de Easton (vide 1.1.33), a seguinte asserção é consistente com ZFC:

$$
(\dagger) \quad \mathfrak{c}=\boldsymbol{\aleph}_{81}+(\forall n \in \omega)\left(2^{\aleph_{n+1}}=\boldsymbol{\aleph}_{\omega+n+1}\right)+2^{\boldsymbol{\aleph}_{\omega}}=\boldsymbol{\aleph}_{\omega+\omega+1}
$$

Se assumimos $(\dagger)$, obtemos as seguintes condições para a existência de um espaço topológico $X$ tal que $|X|=\boldsymbol{\aleph}_{\omega}$ e $w(X)=\lambda$ :

\begin{tabular}{|ll|}
\hline Estrutura de $X$ & Condição necessária e suficiente sobre $\lambda$ \\
\hline \hline Espaço topológico & $\boldsymbol{\aleph}_{1} \leq \lambda \leq \boldsymbol{\aleph}_{\omega+\omega+1}$ \\
Grupo topológico & $\boldsymbol{\aleph}_{1} \leq \lambda \leq \boldsymbol{\aleph}_{\omega+\omega+1}$ \\
Espaço enumeravelmente com- & $\boldsymbol{\aleph}_{1} \leq \lambda \leq \boldsymbol{\aleph}_{\omega+\omega}$ \\
pacto ou pseudocompacto & $\boldsymbol{\aleph}_{1} \leq \lambda<\boldsymbol{\aleph}_{\omega+\omega}$ \\
Grupo pseudocompacto & \\
\hline
\end{tabular}

\subsection{A questão de van Douwen}

Observemos que a afirmação ii) do teorema 4.1.5 e a proposição 4.1.4 estabelecem condições para espaços pseudocompactos, assim, resulta natural se perguntar se essas restrições podem ser melhoradas tratando-se de espaços enumeravelmente compactos. Em relação a isto, van Douwen [27] propôs a seguinte questão:

Questão 4.3. Se X é um grupo infinito (ou um espaço homogêneo) enumeravelmente compacto, tem-se $|X|^{\omega}=|X|$ ? Pelo menos, é $\operatorname{cf}(|X|) \neq \omega$ ?

Sob GCH, qualquer cardinal limite é fortemente limite, daí, pelo corolário 4.1.8, todo espaço homogêneo e pseudocompacto $X$ satisfaz $\operatorname{cf}(|X|) \neq \omega$ logo temos resposta afirmativa à questão anterior. 
A continuação, detalharemos a construção feita por Tomita, via forcing, de um grupo enumeravelmente compacto sem seqüências não triviais convergentes cuja cardinalidade tem cofinalidade enumerável dando resposta negativa à questão 4.3 e estabelecendo assim um resultado de consistência.

Dado $n \in \omega$, se supomos que existe um grupo enumeravelmente compacto de cardinalidade $\boldsymbol{\aleph}_{n}$ então, pela proposição 4.1.4, teria-se que $\boldsymbol{\aleph}_{n} \geq \mathfrak{c}$, e $\operatorname{assim}\left(\boldsymbol{\aleph}_{n}\right)^{\omega}=\boldsymbol{\aleph}_{n}, \operatorname{logo} \operatorname{cf}\left(\boldsymbol{\aleph}_{n}\right) \neq \omega$, o que mostra que nenhum contraexemplo a questão 4.3 pode ter cardinalidade $\boldsymbol{\aleph}_{n}$. Daí, o menor tamanho para um tal grupo, devia ser $\boldsymbol{\aleph}_{\omega}$, e, de fato, o contraexemplo exibido por Tomita em [67] possui dita cardinalidade.

Lema 4.2.1. Se $H$ é um subgrupo de $2^{\mathrm{c}}$ tal que para cada $\left\{h_{n}: n \in \omega\right\} \in[H]^{\omega}$ existe $\alpha<\mathfrak{c}$ tal que o conjunto de restrições $\left\{h_{n} \uparrow_{[\alpha, c)}: n \in \omega\right\}$ tem $0 \in 2^{[\alpha, c)}$ como ponto de acumulação, então se $L=\left\{x \in 2^{\mathfrak{c}}: \operatorname{supp} x\right.$ é limitado em $\left.\mathfrak{c}\right\}$ o grupo $G=\langle H \cup L\rangle=H+L$ é um grupo enumeravelmente compacto de cardinalidade $|H|+2^{<\mathrm{c}}$.

Demonstração. Se $\left\{y_{n}: n \in \omega\right\}$ é uma seqüência no grupo $G$, para cada $n \in \omega$ existem $h_{n} \in H$, $z_{n} \in L$ tais que $y_{n}=h_{n}+z_{n}$. Como $z_{n}=y_{n}-h_{n} \in L$, para cada $n \in \omega$ podemos tomar $\alpha_{n}<\mathfrak{c t a l}$ que supp $\left\{z_{n}\right\} \subseteq \alpha_{n}$. Como $\operatorname{cf}(\mathfrak{c})>\omega, \sup \left\{\alpha_{n}: n \in \omega\right\}=\alpha<\mathfrak{c}$, de modo que para cada $n \in \omega$, $\operatorname{supp}\left\{z_{n}\right\} \subseteq \alpha$, isto é, para cada $n \in \omega,\left(y_{n}-h_{n}\right) \uparrow_{[\alpha, c)}=0 \in 2^{[\alpha, c)}$. Sem perda de generalidade, passando talvez a uma subseqüência, podemos supor que ou $\left\{h_{n}: n \in \omega\right\}$ é uma seqüência com valor constante $h \in H$ ou é uma seqüência injetora.

No primeiro caso $\left\{z_{n} \uparrow_{\alpha}: n \in \omega\right\}=\left\{y_{n}-h \uparrow_{\alpha}: n \in \omega\right\} \subseteq 2^{\alpha}$ tem um ponto de acumulação $x^{\prime} \in 2^{\alpha}$, pois $2^{\alpha}$ é compacto. Em conseqüência, $x=x^{\prime}+h \uparrow_{\alpha}$ é um ponto de acumulação da seqüência $\left\{y_{n} \uparrow_{\alpha}: n \in \omega\right\}$. Como $\left\{z_{n} \uparrow_{[\alpha, c)}: n \in \omega\right\}$ é a seqüência constante com valor $0 \in 2^{[\alpha, c)}$, temos que $\left\{y_{n} \uparrow_{[\alpha, c)}: n \in \omega\right\}=\left\{h_{n} \uparrow_{[\alpha, c)}: n \in \omega\right\}$, logo possui valor constante $h \uparrow_{[\alpha, c)}$. Daí, $x \cup h \uparrow_{[\alpha, c)}=\left(x^{\prime}+h \uparrow_{\alpha}\right) \cup h \uparrow_{[\alpha, c)}=x^{\prime} \cup 0 \uparrow_{[\alpha, c)}+h \in G$ é ponto de acumulação da seqüência $\left\{y_{n}: n \in \omega\right\}$.

No segundo caso, pela hipótese, existe $\beta>\alpha$ tal que o segmento final $\left\{h_{n} \uparrow_{[\beta, c)}: n \in \omega\right\}$ tem a $0 \uparrow_{[\beta, c)}$ como ponto de acumulação. Como $2^{\beta}$ é compacto, existe um ponto de acumulação $y \in 2^{\beta}$ da seqüência $\left\{y_{n} \uparrow_{\beta}: n \in \omega\right\}$; daí, $y \cup 0 \uparrow_{[\beta, c)} \in L \subseteq G$ é ponto de acumulação da seqüência $\left\{y_{n} \uparrow_{\beta} \cup h_{n} \uparrow_{[\beta, c)}: n \in \omega\right\}=\left\{y_{n} \uparrow_{\beta} \cup y_{n} \uparrow_{[\beta, c)}\right\}=\left\{y_{n}: n \in \omega\right\}$, pois $z_{n} \uparrow_{[\beta, c)}=0 \in 2^{[\beta, c)}$ para todo $n \in \omega$.

Portanto, o grupo $G$ é enumeravelmente compacto. Claramente, $L$ codifica todos os subconjuntos de c limitados, logo $|L|=2^{<\mathfrak{c}}$ e assim $|G|=|H|+2^{<\mathfrak{c}}$.

Tomita em [67] salienta que para cada $\kappa \leq \mathfrak{c}$ é possível construir um grupo topológico de cardinalidade $\kappa$ usando $\mathrm{MA}(\kappa)$, mas que ainda MA não é suficiente para construir um 
grupo de cardinalidade $\boldsymbol{\aleph}_{\omega}$. De fato, GCH implica MA e, pelos comentários iniciais, sob GCH todo grupo pseudocompacto não pode ter cardinalidade de cofinalidade enumerável, em particular, sob GCH não existe um grupo enumeravelmente compacto de tamanho $\boldsymbol{\aleph}_{\omega}$. A seguir, detalharemos o modelo obtido por forcing no qual existe um grupo enumeravelmente compacto de cardinalidade $\boldsymbol{\aleph}_{\omega}$.

\subsubsection{Um grupo enumeravelmente compacto de cardinalidade $\aleph_{\omega}$}

Assumamos $\mathrm{CH}$ e seja $\kappa>\boldsymbol{\aleph}_{1}$ um cardinal regular. Fixemos uma enumeração $\left\{f_{\xi}: \xi<\kappa\right\}$ de todas as seqüências injetoras em $[\kappa]^{<\omega}$.

Como antes, se $F \in[\kappa]^{<\omega}$ e $\left\{z_{\mu}: \mu \in F\right\} \subseteq 2^{\kappa}$ denotamos $z_{F}=\sum_{\mu \in F} z_{\mu}$.

Começamos por definir a noção de forcing que permitirá a construção:

Definição 4.2.2. Seja $\mathbb{P}$ o conjunto formado pelas triplas $p=\left(\alpha_{p},\left\{x_{\eta}^{p}: \eta \in E_{p}\right\},\left\{A_{\delta}^{p}: \delta \in D_{p}\right\}\right)$ onde

1. $\alpha_{p} \in \omega_{1}$;

2. $D_{p} \in[\kappa]^{\omega}, E_{p}=D_{p} \cup \bigcup\left\{f_{\delta}(n): n \in \omega, \delta \in D_{p}\right\}$;

3. $x_{\eta}^{p} \in 2^{\alpha_{p}}$ para cada $\eta \in E_{p}$;

4. $A_{\delta}^{p} \in[\omega]^{\omega}$ para cada $\delta \in D_{p}$.

Em $\mathbb{P}$ dizemos que $p \leq q$ se:

a. $\alpha_{q} \leq \alpha_{p}, D_{q} \subseteq D_{p}, E_{q} \subseteq E_{p}$;

b. para todo $\eta \in E_{q}$ temos $x_{\eta}^{q} \subseteq x_{\eta}^{p}$;

c. para cada $\delta \in D_{p}, A_{\delta}^{p} \subseteq^{*} A_{\delta}^{q} \mathrm{e}$,

d. para cada $\delta \in D_{p}$, a seqüência $\left\{x_{f_{\delta}(n)} \uparrow_{\left[\alpha_{q}, \alpha_{p}\right)}: n \in A_{\delta}^{p}\right\}$ converge ao ponto $0 \uparrow_{\left[\alpha_{q}, \alpha_{p}\right)} \in 2^{\left[\alpha_{q}, \alpha_{p}\right)}$.

Lema 4.2.3. O conjunto parcialmente ordenado $(\mathbb{P}, \preceq)$ é enumeravelmente fechado e satisfaz a condição $\omega_{2}$-c.c.

Demonstração. Seja $\left\{p_{n}: n \in \omega\right\}$ uma seqüência decrescente de elementos em $\mathbb{P}$, onde, para cada $n \in \omega, p_{n}=\left(\alpha_{n},\left\{x_{\eta}^{n}: \eta \in E_{n}\right\},\left\{A_{\delta}^{n}: \delta \in D_{n}\right\}\right)$.

Tomemos 


$$
\alpha_{\omega}=\sup \left\{\alpha_{n}: n \in \omega\right\}, \quad D_{\omega}=\bigcup_{n \in \omega} D_{n}, E_{\omega}=\bigcup_{n \in \omega} E_{n}, x_{\eta}^{\omega}=\bigcup_{\substack{\eta \in E_{n} \\ n \in \omega}} x_{\eta}^{n}
$$

Para cada $\delta \in D_{\omega}$, seja $A_{\delta}^{\omega} \in[\omega]^{\omega}$ tal que para cada $n \in \omega$, se $\delta \in D_{n}$, então $A_{\delta}^{\omega} \subseteq^{*} A_{\delta}^{n}$. Por fim, seja $p=\left(\alpha_{\omega},\left\{x_{\eta}^{\omega}: \eta \in E_{\omega}\right\},\left\{A_{\delta}^{\omega}: \delta \in D_{\omega}\right\}\right)$.

Afirma-se que $p \in \mathbb{P}$. Com efeito, dado que $\operatorname{cf}\left(\omega_{1}\right) \neq \omega, \alpha_{\omega}<\omega_{1}$; agora, como para cada $n \in \omega$ os conjuntos $D_{n}$ e $E_{n}$ são enumeráveis, $D_{\omega} \in[\kappa]^{\omega}$ e $E_{\omega} \in[\kappa]^{\omega}$ pois $\kappa>\omega$. De acordo com a construção, as outras condições para pertencer à ordem $\mathbb{P}$ são satisfeitas trivialmente. É obvio que para cada $n \in \omega, p_{\omega} \leq p_{n}$, logo $\mathbb{P}$ é enumeravelmente fechada.

Seja $\left\{p_{\mu}: \mu \in \omega_{2}\right\} \subseteq \mathbb{P}$ de maneira tal que para cada $\mu, \gamma \in \omega_{2}, p_{\mu} \neq p_{\gamma}$. Como $\left|E_{\mu}\right|=\omega<\omega_{1}$ e $\left|\left\{E_{\mu}: \mu<\omega_{2}\right\}\right|=\omega_{2} \geq \omega_{1}$ pelo lema do $\Delta$-sistema, existem $I \in\left[\omega_{2}\right]^{\omega_{2}}$ e $E \in[\kappa]^{\leq \omega}$ tais que $E_{\mu} \cap E_{\beta}=E$ para todo $\{\mu, \beta\} \in[\kappa]^{2}$. Como $|I|=\omega_{2}>\omega_{1}$ e $\left\{\alpha_{p}: p \in I\right\} \subseteq \omega_{1}$ existem $J \subseteq I$ e $\alpha \in \omega_{1}$ tais que $|J|=\omega_{2}$ e para todo $\mu \in J, \alpha_{\mu}=\alpha$.

Além disso, como $|E| \leq \omega$ e $\alpha \in \omega_{1}$, existem só $\mathfrak{c}=\omega_{1}$ funções de $E$ a $2^{\alpha}$ e existem também só $\mathfrak{c}=\omega_{1}$ funções de $E$ a $[\omega]^{\omega}$; desta maneira, podemos supor que para cada par $\{\mu, \beta\} \in[J]^{2}$, e $\eta \in E, x_{\eta}^{\beta}=x_{\eta}^{\mu}$ e, para todo $\delta \in D_{\mu} \cap D_{\beta} \subseteq E, A_{\delta}^{\beta}=A_{\delta}^{\mu}$.

Sejam $\mu, \beta \in J$ distintos; se

$$
p=\left(\alpha,\left\{x_{\eta}^{\mu}: \eta \in E_{\mu}\right\} \cup\left\{x_{\eta}^{\beta}: \eta \in E_{\beta} \backslash E\right\},\left\{A_{\delta}^{\mu}: \delta \in D_{\mu}\right\} \cup\left\{A_{\delta}^{\beta}: \delta \in D_{\beta} \backslash D_{\mu}\right\}\right)
$$

então naturalmente $p \in \mathbb{P}$ e $p \leq p_{\beta}, p \leq p_{\mu}$. Em conseqüência, não existem anticadeias de tamanho $\omega_{2}$ em $\mathbb{P}$, isto é, $\mathbb{P}$ é $\omega_{2}$-c.c.

Prosseguimos definindo alguns conjuntos densos da ordem $\mathbb{P}$. Para cada $\phi \in \operatorname{Fn}(\kappa, 2)$ e $\alpha \in \omega_{1}$, seja

$$
D_{\phi, \alpha}=\left\{p \in \mathbb{P}: \operatorname{dom} \phi \subseteq D_{p} \text { e existe } \gamma \in\left[\alpha, \alpha_{p}\right) \text { tal que para todo } \xi \in \operatorname{dom} \phi, \phi(\xi)=x_{\xi}^{p}(\gamma)\right\}
$$

Lema 4.2.4. A família $\mathscr{D}=\left\{D_{\phi, \alpha}: \phi \in \mathrm{Fn}(\kappa, 2), \alpha<\omega_{1}\right\}$ está formada por subconjuntos densos na ordem $\mathbb{P}$.

Demonstração. Seja $q \in \mathbb{P}$ e fixemos $\phi \in \operatorname{Fn}(\kappa, 2), \alpha<\omega_{1}$. Tomemos $\alpha_{r}=\max \left\{\alpha, \alpha_{q}\right\}$, $D_{r}=D_{q} \cup \operatorname{dom} \phi, E_{r}=D_{r} \cup \bigcup\left\{f_{\delta}(n): \delta \in D_{r}, n \in \omega\right\}$.

Para cada $\eta \in E_{r}$, e $\delta \in D_{r}$ tomemos

$$
x_{\eta}^{r}=\left\{\begin{array}{ll}
x_{\eta}^{q} \cup 0 \uparrow_{\left[\alpha_{q}, a_{r}\right)} & \text { se } \eta \in E_{q} \\
0 & \text { se } \eta \in E_{r} \backslash E_{q}
\end{array} \quad \text { e } \quad A_{\delta}^{r}= \begin{cases}A_{q}^{r} & \text { se } \delta \in D_{q} \\
\omega & \text { se } \delta \in D_{r} \backslash D_{q}\end{cases}\right.
$$


Se tomarmos $r=\left(\alpha_{r},\left\{x_{\eta}^{r}: \eta \in E_{r}\right\},\left\{A_{\delta}^{r}: \delta \in D_{r}\right\}\right)$ naturalmente $r \in \mathbb{P}$ e $r \leq q$. A idéia agora é achar uma extensão de $r$ em $D_{\phi, \alpha}$. Seja $\alpha_{p}=\alpha_{r}+1, D_{p}=D_{r}$ e $E_{p}=E_{r}$. Definiremos uma função $\psi: E_{p} \rightarrow 2$ de maneira tal que para cada $\eta \in E_{p}, \psi(\eta)=x_{\eta}^{p}\left(\alpha_{r}\right)$.

Primeiro, fixemos uma enumeração $\left\{\beta_{n}: n \in \omega\right\}$ do conjunto $D_{p}$, de modo tal que para cada $\delta \in D_{p},\left\{n \in \omega: \beta_{n}=\delta\right\} \in[\omega]^{\omega}$.

Seja $F_{0}=$ dom $\phi$ e definimos $\psi \uparrow_{F_{0}}=\phi$. Usando um argumento indutivo vamos definir para cada $n \in \omega, \psi \uparrow_{F_{n}}$ e $k_{n} \in \omega$ satisfazendo as seguintes condições:

$$
\begin{aligned}
& \diamond F_{n} \cup f_{\beta_{n}}\left(k_{n}\right) \subseteq F_{n+1}, \\
& \diamond k_{n} \in A_{\beta_{n}}^{r}, k_{n}<k_{n+1}, \mathrm{e}, \\
& \diamond \sum\left\{\psi(\mu): \mu \in f_{\beta_{n}}\left(k_{n}\right)\right\}=0
\end{aligned}
$$

Suponhamos que para cada $m \leq n, F_{m}, \psi \uparrow_{F_{m}}$ e $k_{m-1}$ tem sido definidos satisfazendo as condições anteriores. Seja $k_{n} \geq k_{n-1}$ tal que $k_{n} \in A_{\beta_{n}}^{r}$ e $f_{\beta_{n}}\left(k_{n}\right) \backslash F_{n} \neq \varnothing$.

Tomemos $F_{n+1}=F_{n} \cup f_{\beta_{n}}\left(k_{n}\right)$; por se tratar de uma função com valores ao grupo booleano 2, a função $\psi$ pode ser definida sobre o conjunto $F_{n+1} \backslash F_{n}$ de maneira que $\sum\{\psi(\mu): \mu \in$ $\left.f_{\beta_{n}}\left(k_{n}\right)\right\}=0$.

No final da indução, teremos definido $\psi$ sobre o conjunto $F=\bigcup_{n \in \omega} F_{n}$. Para cada $\mu \in E_{p} \backslash F$, tomemos $\psi(\mu)=0$. Dados $\eta \in E_{p}$ e $\delta \in D_{p}$, sejam

$$
x_{\eta}^{p}:=x_{\eta}^{r} \cup\left\{\left(\alpha_{r}, \psi(\eta)\right)\right\} \text { e } A_{\delta}^{p}:=\left\{k_{n}: \beta_{n}=\delta\right\}
$$

É claro que $p=\left(\alpha_{p},\left\{x_{\eta}^{p}: \eta \in E_{p}\right\},\left\{A_{\delta}^{p}: \delta \in D_{p}\right\}\right) \in \mathbb{P}$; mostremos que $p \in D_{\phi, \alpha}$ e $p \leq q$.

Como $\alpha_{p}=\alpha_{r}+1, D_{p}=D_{r}$ e $E_{r}$, as três primeiras condições que descrevem a relação $p \leq r$ sobre $\mathbb{P}$, estão satisfeitas. Do mesmo modo, para cada $\eta \in E_{r}=E_{p}$ temos que $x_{\eta}^{p} \uparrow_{a_{r}}=x_{\eta}^{r}$ e para cada $\delta \in D_{p}=D_{r}$, de acordo com a construção indutiva, se satisfaz $A_{\delta}^{p} \subseteq A_{\delta}^{r}$.

Dado $\delta \in D_{p}$, se consideramos a seqüência $\left\{x_{f_{\delta}(n)}^{p} \uparrow_{\left[\alpha_{r}, a_{r+1}\right)}: n \in A_{\delta}^{p}\right\}$, temos, para cada $n \in A_{\delta}^{p}:$

$$
\begin{aligned}
x_{f_{\delta}(n)}^{p}\left(\alpha_{r}\right) & =x_{f_{\delta}\left(k_{n}\right)}^{p}\left(\alpha_{r}\right) \\
& =x_{f_{\beta_{n}}\left(k_{n}\right)}^{p}\left(\alpha_{r}\right) \\
& =\sum_{\mu \in f_{\beta}\left(k_{n}\right)} x_{\mu}\left(\alpha_{r}\right) \\
& =\sum_{\mu \in f_{\beta}\left(k_{n}\right)} \psi(\mu)=0
\end{aligned}
$$

Portanto, $p \leq r \leq q$. Visto que $\operatorname{dom} \phi \subseteq D_{r}, \alpha_{p}>\alpha_{r} \geq \alpha$ e para cada $\xi \in \operatorname{dom} \phi=F_{0}$ temos $x_{\xi}^{p}\left(\alpha_{r}\right)=\psi(\xi)=\phi(\xi)$, concluímos que $p \in D_{\phi, \alpha} ;$ portanto, cada membro da coleção $\mathscr{D}$ é denso 
em $\mathbb{P}$.

Teorema 4.2.5. Se $\mathcal{M}$ é um modelo de $\mathrm{CH} e(\kappa>\mathfrak{c})^{\mathcal{M}}$ é um cardinal regular, então existe uma noção de forcing enumeravelmente fechada satisfazendo a condição $\omega_{2}$-c.c. tal que para cada cardinal $\lambda \in[\mathfrak{c}, \kappa]$, na extensão $\mathcal{M}[\mathbb{G}]$ existe um grupo enumeravelmente compacto $G$ cuja cardinalidade é $\lambda$.

Demonstração. Suponhamos $\mathrm{CH}$ e seja $\kappa>\mathfrak{c}$ um cardinal regular. Seja $\mathbb{P}$ como na definição 4.2.2. Pelo lema 4.2.3, $\mathbb{P}$ é uma ordem enumeravelmente fechada que satisfaz $\omega_{2}$-c.c., logo, trata-se de uma ordem que preserva cardinalidades e cofinalidades.

Seja $\mathbb{G} \in \mathcal{M}[\mathbb{G}]$ um filtro genérico para a ordem parcial $\mathbb{P}$. Para cada $\xi \in \kappa$, definimos

$$
x_{\xi}=\bigcup_{\substack{p \in \mathbb{G} \\ \xi \in D_{p}}} x_{\xi}^{p}
$$

Seja $\phi=\{(\xi, 0)\} \in \mathrm{Fn}(\kappa, 2)$. Observemos que para cada $\alpha<\omega_{1}$ existe $p \in \mathbb{G} \cap D_{\phi, \alpha}$ satisfazendo $\alpha_{p}>\alpha$ e $\xi \in D_{p}$; daí, uma vez que $\operatorname{dom} x_{\xi}^{p}=\alpha_{p}>\alpha$, obtemos $\alpha \in \operatorname{dom} x_{\xi}$ para cada $\alpha<\omega_{1}$ e assim, $\operatorname{dom} x_{\xi}=\omega_{1}$, isto é, $x_{\xi} \in 2^{\omega_{1}}$ para cada $\xi<\kappa$.

Afirmamos que o conjunto de funções $X=\left\{x_{\xi}: \xi<\kappa\right\}$ é linearmente independente. Primeiro, dado $F \in[\kappa]^{<\omega} \backslash\{\varnothing\}$, podemos definir de maneira conveniente uma função $\vartheta: F \rightarrow$ 2 tal que $\sum_{\mu \in F} \vartheta(\mu) \neq 0$. Como $\mathbb{G}$ é genérico, podemos também tomar $p \in \mathbb{G} \cap D_{\vartheta, \omega}$, mas daí, existe $\gamma \in\left[\omega, \alpha_{p}\right)$ tal que para todo $\xi \in F, \vartheta(\xi)=x_{\xi}^{p}(\gamma)$, logo

$$
\begin{aligned}
x_{F}(\gamma) & =\sum_{\xi \in F} x_{\xi}^{p}(\gamma) \\
& =\sum_{\xi \in F} \vartheta(\xi) \neq 0
\end{aligned}
$$

ou seja, $x_{F} \neq 0 \in 2^{\omega_{1}}$. Percebendo que cada conjunto finito não vazio $F \subseteq \kappa$ codifica uma combinação linear não nula -uma soma pela estrutura booleana de $2^{\kappa}$ - de elementos da família $X$, segue o resultado.

Seja $I \subseteq \kappa$ não vazio. Afirma-se que o subgrupo $H_{I}=\left\langle x_{\xi}: \xi \in I\right\rangle \subseteq 2^{\kappa}$ satisfaz as condições do lema 4.2.1; para mostrar isto, tomemos uma seqüência $\left\{y_{n}: n \in \omega\right\} \in\left[H_{I}\right]^{\omega}$. Como $H_{I}=\left\langle x_{\xi}: \xi\langle\mathfrak{c}\rangle\right.$, para cada $n \in \omega$ podemos associar um conjunto de índices $f(n) \in$ $[I]^{<\omega}$ tal que $y_{n}=\sum_{\mu \in f(n)} x_{\mu}=x_{f(n)}$, obtendo desta maneira uma função $f: \omega \rightarrow[I]^{<\omega}$. Dado que $X$ é linearmente independente e a seqüência escolhida é injetora, segue que $f$ é injetora. Observemos que em particular $f$ é uma seqüência injetora em $[\kappa]^{<\omega}$; assim, como a noção de 
forcing $\mathbb{P}$ não adiciona novos subconjuntos enumeráveis ao modelo $\mathbb{M}$, existe $\mu<\kappa$ tal que $f=f_{\mu}$, lembrando que $\left\{f_{\mu}: \mu<\kappa\right\}$ enumera todas as seqüências injetoras em $[\kappa]^{<\omega}$.

Seja $\varrho \in \operatorname{Fn}(\kappa, 2)$ tal que $\mu \in$ dom $\varrho$. Visto que para cada $\alpha<\omega_{1}$ o conjunto $D_{\varrho, \alpha}$ é denso em $\mathbb{P}$, podemos tomar $p \in \mathbb{G} \cap D_{\mathrm{o}, \mathrm{a}}$, logo, em particular, $\mu \in D_{p}$. Se $F$ é um subconjunto finito de $\left[\alpha_{p}, \omega_{1}\right)$, e $\beta=\max F$, dado que para qualquer função $\varphi \in \mathrm{Fn}(\kappa, 2)$ o conjunto $D_{\varphi, \beta}$ é denso em $\mathbb{P}$, podemos achar $q \in D_{\varphi, \beta} \cap \mathbb{G}$ tal que $q \leq p$. Como $\mu \in D_{p} \subseteq D_{q}$, de acordo com a definição da ordem, a seqüência $\left\{x_{f_{\mu}(n)}^{q} \uparrow_{\left[\alpha_{p}, \alpha_{q}\right)}: n \in A_{\mu}^{q}\right\}$ converge a $0 \in 2^{\left[\alpha_{p}, \alpha_{q}\right)}$, logo, o conjunto $\left\{n \in A_{\mu}^{q}: x_{f_{\mu}(n)}^{q} \uparrow_{F}=0 \uparrow_{F}\right\}$ é cofinito. Visto que $x_{f_{\mu}(n)}^{q} \uparrow_{F}=x_{f(n)}^{q} \uparrow_{F} x_{f(n)} \uparrow_{F}=\sum_{v \in f(n)} x_{v} \uparrow_{F}=y_{n} \uparrow_{F}$ temos

$$
\begin{aligned}
A_{\mu}^{q} & \subseteq^{*}\left\{n \in A_{\mu}^{q}: x_{f_{\mu}(n)}^{q} \uparrow_{F}=0 \uparrow_{F}\right\} \\
& =\left\{n \in A_{\mu}^{q}: y_{n} \uparrow_{F}=0 \uparrow_{F}\right\} \\
& \subseteq\left\{n \in \omega: y_{n} \uparrow_{F}=0 \uparrow_{F}\right\}
\end{aligned}
$$

Assim, $0 \in 2^{\left[\alpha_{p}, \alpha_{q}\right)}$ é ponto de acumulação de $\left\{y_{n} \uparrow_{\left[\alpha_{p}, \alpha_{q}\right)}: n \in \omega\right\}$; pelo lema 4.2 .1 obtém-se que o grupo $L+H_{I}$ é enumeravelmente compacto e de cardinalidade $\left|H_{I}\right|+2^{<c}$. Note que $H_{I}$ e $I$ possuem a mesma cardinalidade pois identificamos elementos do grupo $H_{I}$ com subconjuntos finitos de $I$; além disso, ao assumirmos $\mathrm{CH}$ no modelo $\mathbb{M}$ e ao $\mathbb{P}$ satisfazer $\omega_{2}$-c.c., segue que $2^{<\mathfrak{\imath}}=\mathfrak{c}$. Portanto, $\left|L+H_{I}\right|=|I|+\mathfrak{c}=|I|$ o que conclui a prova.

Corolário 4.2.6. É consistente $\mathfrak{c}=\boldsymbol{\aleph}_{1}<\boldsymbol{\aleph}_{\omega}$ com a existência de um grupo enumeravelmente compacto de cardinalidade $\boldsymbol{\aleph}_{\omega}$.

Demonstração. Seja $\mathcal{M}$ um modelo de GCH. Seja $\left(\kappa=\boldsymbol{\aleph}_{\omega+1}\right)^{\mathcal{M}}$. Pelo teorema 4.2.5, como $\kappa$ é regular, na extensão $\mathcal{M}[\mathbb{G}]$, para cada $\lambda \in\left[\mathfrak{c}, \boldsymbol{\aleph}_{w+1}\right]$ existe um grupo enumeravelmente compacto de cardinalidade $\lambda$. Seja $\left(\lambda=\boldsymbol{\aleph}_{\omega}\right)^{\mathcal{M}}$. Como a noção de forcing empregada é enumeravelmente fechada, preserva cardinais abaixo de $\boldsymbol{\aleph}_{2}$ e assim, $(\mathrm{CH})^{\mathcal{M}[\mathbb{G}]}$. Além disso, a noção de forcing é $\omega_{2}$-c.c., logo preserva cardinais acima de $\boldsymbol{\aleph}_{2}$ e, portanto, $\left(\lambda=\boldsymbol{\aleph}_{w}\right)^{\mathcal{M}[G]}$. Conseqüentemente, em $\mathcal{M}[\mathbb{G}] \mathrm{CH}$ vale e existe um grupo enumeravelmente compacto cuja cardinalidade tem cofinalidade enumerável.

De acordo com os resultados da seção anterior e como conseqüência do corolário anterior, neste ponto aparece um resultado de independência:

Teorema 4.2.7. A existência de grupos enumeravelmente compactos cuja cardinalidade tem cofinalidade enumerável é independente de ZFC. 
Demonstração. Por uma parte, pelo corolário 4.1.8 num modelo $\mathcal{M}$ de $\mathrm{ZFC}+\mathrm{GCH}$ não existem espaços homogêneos cuja cardinalidade tenha cofinalidade enumerável. De outro lado, pelo teorema 4.2.6 obtemos um modelo $\mathcal{N}$ de $\mathrm{ZFC}+\mathrm{CH}$ onde existe um grupo cuja cardinalidade tem cofinalidade enumerável. Em conseqüência, a asserção é independente de ZFC.

\subsubsection{Um grupo enumeravelmente compacto de peso $\aleph_{\omega}$}

Sirota [58] respondendo a uma questão proposta por Arkhangel'skiǐ, construiu um grupo pseudocompacto sem seqüências não triviais convergentes de peso $\kappa$ para qualquer cardinal infinito $\kappa$ satisfazendo $\kappa=\kappa^{\omega}$. Complementando o resultado obtido por van Douwen sob GCH acerca da cardinalidade de um grupo pseudocompacto, Malykhin e Shapiro [51] mostraram que sob GCH, os únicos pesos possíveis para grupos pseudocompactos sem seqüências não triviais convergentes satisfazem $\kappa=\kappa^{\omega}$, e num modelo de forcing, os autores mostraram a existência de um grupo pseudocompacto de peso $\boldsymbol{\aleph}_{1}<\left(\boldsymbol{\aleph}_{1}\right)^{\omega}$. Como $\omega_{1}$ tem cofinalidade não enumerável, é natural perguntar se um grupo pseudocompacto sem seqüências não triviais convergentes pode ter peso de cofinalidade enumerável, e, se a pseudocompacidade pode ser substituída pela compacidade enumerável.

Com referência a essa questão, no trabalho [67] Tomita proporcionou um resultado de independência, ao mostrar de maneira consistente a existência de um grupo abeliano de van Douwen de cardinalidade $\mathfrak{c}$ e peso $\boldsymbol{\aleph}_{\omega}$. Neste parágrafo ser åo apresentados os detalhes dessa construção.

O seguinte teorema mostra uma maneira de aumentar o peso de grupos de van Douwen de cardinalidade $\mathfrak{c}$.

Teorema 4.2.8. Se existe uma topologia de grupo enumeravelmente compacta e sem seqüências não triviais convergentes sobre um grupo abeliano livre infinito $G$, então para cada $\kappa \in\left[\mathfrak{c}, 2^{\mathfrak{c}}\right]$ existe uma topologia de grupo $\tau_{\kappa}$ enumeravelmente compacta e sem seqüências não triviais convergentes sobre o grupo $\mathrm{A}(\mathfrak{c})$, de modo tal que $w\left(\left(\mathrm{~A}(\mathfrak{c}), \tau_{\kappa}\right)\right)=\kappa$.

Demonstração. Como $G$ é, em particular, pseudocompacto pela proposição 4.1.4 temos que $|G| \geq \mathfrak{c}$. Se $|G|>\mathfrak{c}$, usando o argumento de clossing-off descrito anteriormente, obtemos um subgrupo $G^{\prime} \leq G$ enumeravelmente compacto tal que $\left|G^{\prime}\right|=\mathfrak{c}$. Adicionalmente, notemos que $G^{\prime}$ é livre, logo, podemos assumir sem perda de generalidade que $|G|=\mathfrak{c}$. Como $G$ é abeliano livre e enumeravelmente compacto, podemos identificar a $G$ com um subgrupo de $\mathbb{T}^{\lambda}$ para algum cardinal $\lambda$ mediante um isomorfismo de grupos topológicos. Note-se que $[G]^{<\omega}=\mathfrak{c}$, logo podemos projetar $G$ em $\mathbb{T}^{\theta}$ de maneira injetiva para algum $\theta \leq \mathfrak{c}$; como $G$ é de van 
Douwen, a imagem sob dita projeção constitui um grupo abeliano livre sem seqüências não triviais convergentes, assim, podemos assumir que $w(G) \leq \theta \leq \mathfrak{c}$.

Seja $\left\{y_{\xi}: \xi<\mathfrak{c}\right\}$ um conjunto independente de geradores de $G$. Seja $P=\{\kappa 2: \kappa<\mathfrak{c}\}$ o conjunto de todos os ordinais pares em $\mathfrak{c}$. Usando $P$, o conjunto de seqüências injetoras de elementos de $\mathrm{Fn}(\mathfrak{c}, \mathbb{Z} \backslash\{0\}) \backslash\{\varnothing\}$ é indexado, digamos $\left\{f_{\xi}: \xi \in P\right\}$. Adicionalmente podemos supor que para cada $\xi \in P, \bigcup_{n \in \omega} \operatorname{dom} f_{\xi}(n) \subseteq \xi$.

Seja $0<\xi<c$. Por indução, suponhamos que para cada $\mu<\xi$ temos escolhido $x_{\mu} \in G$ satisfazendo as seguintes duas condições:

a. o conjunto $\left\{x_{\beta}: \beta<\mu\right\}$ é linearmente independente, e,

b. se $\mu \in P$, então $x_{\mu}$ é um ponto de acumulação da seqüência $\left\{x_{f_{\mu}(n)}: n \in \omega\right\}$.

Afirma-se que $x_{\xi}$ pode ser escolhido de forma tal que a hipótese de indução é satisfeita. De fato, suponhamos que $H=\left\langle x_{\beta}: \beta<\xi\right\rangle$. Como $\left\{y_{\eta}: \eta<\mathfrak{c}\right\}$ é um conjunto livre de geradores de $G \supset H$, para cada $h \in H$ associa-se um único subconjunto $J_{h} \in[\mathrm{c}]^{<\omega}$ de modo tal que $h \in\left\langle y_{\beta}: \beta \in J_{h}\right\rangle$. Seja $J=\bigcup_{h \in H} J_{h}$; o elemento $x_{\S}$ é escolhido como segue:

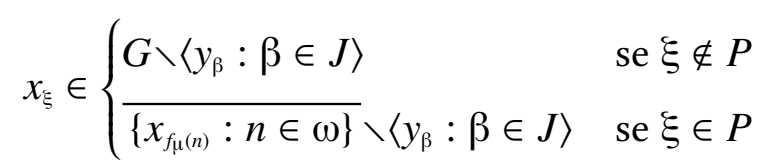

Como $\left|\overline{\left\{x_{f_{u}(n)}: n \in \omega\right\}}\right|=\mathfrak{c}$ e $\left|\left\langle y_{\beta}: \beta \in J\right\rangle\right|=|J| \leq \xi<\mathfrak{c}$, note-se que $\overline{\left\{x_{f_{u}(n)}: n \in \omega\right\}} \backslash\left\langle y_{\beta}: \beta \in\right.$ $J\rangle \neq \varnothing$. Claramente, o conjunto $\left\{x_{\beta}: \beta<\xi\right\}$ satisfaz as condições a) e b) anteriores.

No final do processo indutivo, obtemos um conjunto $X=\left\{x_{\xi}: \xi<\mathfrak{c}\right\}$ linearmente independente tal que para cada $\xi \in P, x_{\xi}$ é ponto de acumulação da seqüência no grupo gerado por $X$ associada a função $f_{\xi}$. Pela última afirmação, para cada $\xi<\mathfrak{c}$ pode-se tomar um ultrafiltro $p_{\xi} \in \omega^{*}$ de maneira tal que $x_{\xi}=p-\lim _{n \in \omega} x_{f_{\xi}(n)}$.

Fixemos $\kappa \in\left[\theta, 2^{\mathfrak{c}}\right]$ e seja $\left\{z_{\alpha}: \alpha \in \mathfrak{c} \backslash P\right\}$ um subconjunto denso em $\mathbb{T}^{\kappa}$. Dado que todo espaço compacto é, em particular, ultracompacto, para cada $\mu \in P$ podemos definir, de maneira indutiva, $z_{\mu}=p_{\mu}-\lim _{n \in \omega} z_{f_{\mu}(n)}$. Finalmente, seja $G_{\mathrm{\kappa}}=\left\langle\left(x_{\xi}, z_{\xi}\right): \xi\langle\mathfrak{c}\rangle \subseteq G \times \mathbb{T}^{\mathrm{K}}\right.$.

Observe-se que $G_{\kappa}$ é um grupo abeliano de cardinalidade c e, além disso, a coleção $\left\{\left(x_{\xi}, z_{\xi}\right): \xi<\mathfrak{c}\right\}$ é um conjunto livre de geradores para esse grupo. De fato, suponha-se que $0_{\mathbb{T}^{\theta+\kappa}}=\sum_{\mu \in F} a_{\mu}\left(x_{\mu}, z_{\mu}\right)$ onde $F \in[\mathfrak{c}]^{<\omega} \neq \varnothing$, e $a_{\mu} \in \mathbb{Z}$ para cada $\mu \in F$; de acordo com a definição da operação do grupo, $0_{\mathbb{T}^{\theta}}=\sum_{\mu \in F} a_{\mu} x_{\mu}$. Assim, $a_{\mu}=0$ para todo $\mu \in F$, posto que o conjunto $\mathcal{X}$ é independente. Resta provar que $G_{\mathrm{k}}$ é um grupo de van Douwen. 
Suponha-se que $\left\{g_{n}: n \in \omega\right\}=\left\{\left(t_{n}, w_{n}\right): n \in \omega\right\} \subseteq G_{\text {к }}$ é uma seqüência injetora; passando talvez a uma subseqüência, podemos assumir que $\left\{g_{n}: n \in \omega\right\}$ não contém termos do tipo $\left(0_{\mathbb{T}^{\theta}}, 0_{\mathbb{T}^{\kappa}}\right),\left(t, 0_{\mathbb{T}^{\kappa}}\right)$ ou $\left(0_{\mathbb{T}^{\theta}}, w\right)$ para $t \in G, w \in \mathbb{T}^{\kappa}$.

Inicialmente, consideremos o caso em que para cada $n, m \in \omega$ distintos, $t_{n} \neq t_{m}$ e $w_{n} \neq w_{m}$. Notemos que dado $n \in \omega$, o ponto $g_{n}=\left(t_{n}, w_{n}\right)$ é soma de um número finito de elementos do conjunto $\left\{\left(x_{\xi}, z_{\xi}\right): \xi<\mathfrak{c}\right\}$, logo podemos associar-lhe a esse ponto uma função $f(n) \in$ Fn $(\mathfrak{c}, \mathbb{Z} \backslash\{0\})$ de modo tal que

$$
\begin{aligned}
g_{n}=\left(t_{n}, w_{n}\right) & =\sum_{\mu \in \operatorname{dom} f(n)} f(n)(\mu)\left(x_{\mu}, z_{\mu}\right) \\
& =\sum_{\mu \in \operatorname{dom} f(n)}\left(f(n)(\mu) x_{\mu}, f(n)(\mu) z_{\mu}\right) \\
& =\left(x_{f(n)}, z_{f(n)}\right)
\end{aligned}
$$

Como $\left\{x_{\xi}: \xi<\mathfrak{c}\right\}$ é independente, segue que a correspondência $f: \omega \rightarrow \operatorname{Fn}(\mathfrak{c}, \mathbb{Z} \backslash\{0\})$ é injetora; portanto, existe $\zeta \in P$ tal que $f=f_{\xi}$. De acordo com a construção, observe-se que $x_{\zeta}=p_{\zeta}-\lim _{n \in \omega} x_{f_{\zeta}(n)}$; de outra parte, $z_{\zeta}=p_{\zeta}-\lim _{n \in \omega} z_{f_{\zeta}(n)}$. Como a $p_{\xi}$-compacidade é preservada sob funções contínuas, naturalmente temos

$$
\left(x_{\zeta}, z_{\zeta}\right)=p_{\zeta}-\lim _{n \in \omega}\left(x_{f_{\zeta}(n)}, z_{f_{\zeta}(n)}\right)
$$

de onde $\left(x_{\zeta}, z_{\zeta}\right)$ é um ponto de acumulação da seqüência $\left\{\left(x_{f_{\zeta}(n)}, z_{f_{\zeta}(n)}\right): n \in \omega\right\}=\left\{\left(t_{n}, w_{n}\right): n \in\right.$ $\omega\}$.

Uma segunda situação que pode ocorrer, é que $\left\{t_{n}: n \in \omega\right\} \subseteq G$ contenha uma subseqüência constante $\left\{t_{n_{k}}: k \in \omega\right\}$ tomando o valor $t \in\left\langle x_{\xi}: \xi<\mathfrak{c}\right\rangle$; notemos que nesse caso, necessariamente, $\left\{w_{n_{k}}: k \in \omega\right\} \subseteq \mathbb{T}^{c}$ é uma seqüência injetora. Para cada $k \in \omega$, podemos associar uma função, que possivelmente não é única, $f(k) \in \operatorname{Fn}(\mathfrak{c}, \mathbb{Z} \backslash\{0\})$ de modo que $w_{n_{k}}=\sum_{\mu \in \operatorname{dom} f(k)} f(k)(\mu) z_{\mu}$; note-se que, como antes, a função $f: \omega \rightarrow \operatorname{Fn}(\mathfrak{c}, \mathbb{Z} \backslash\{0\})$ é injetora, $\operatorname{logo}$ existe $\zeta \in P$ tal que $f=f_{\zeta}$. Como $z_{\zeta}=p_{\zeta}-\lim _{k \in \omega} z_{f_{\zeta}(k)}=p_{\zeta}-\lim _{k \in \omega} z_{f(k)}=p_{\zeta}-\lim _{k \in \omega} w_{n_{k}}$, segue que $z_{\zeta}$ é ponto de acumulação da seqüência $\left\{w_{n}: n \in \omega\right\}$. Daí, $\left(t, z_{\zeta}\right) \in G_{\mathrm{\kappa}}$ é ponto de acumulação de $\left\{\left(y_{n}, w_{n}\right): n \in \omega\right\}$.

Agora, se ocorrer que $\left\{w_{n}: n \in \omega\right\}$ contém uma subseqüência constante $\left\{w_{n_{k}}: k \in \omega\right\}$ que toma o valor $w$, necessariamente $\left\{t_{n_{k}}: k \in \omega\right\}$ é uma seqüência injetora no grupo $\left\langle x_{\xi}: \xi \in \mathfrak{c}\right\rangle$; assim, como esse conjunto é linearmente independente, existe uma -desta vez, única- função injetora $f: \omega \rightarrow \operatorname{Fn}(\mathfrak{c}, \mathbb{Z} \backslash\{0\})$ tal que para cada $k \in \omega, t_{n_{k}}=\sum_{\mu \in \operatorname{dom} f(k)} f(k)(\mu) x_{\mu}=x_{f(k)}$. De 
acordo com a enumeração escolhida previamente, existe também $\zeta \in P$ tal que $f=f_{\zeta}$, logo, $x_{\zeta}$ é ponto de acumulação de $\left\{x_{f_{\zeta}(k)}: k \in \omega\right\}=\left\{x_{n_{k}}: k \in \omega\right\}$; em conseqüência, $\left(x_{\zeta}, w\right) \in G_{\mathrm{\kappa}}$ é ponto de acumulação de $\left\{\left(t_{n}, w_{n}\right): n \in \omega\right\}$.

Do anterior, conclui-se que $G_{\mathrm{\kappa}}$ é enumeravelmente compacto. Como $G_{\mathrm{\kappa}}$ contém uma copia de um subgrupo de $G$ que, naturalmente, não admite seqüências não triviais convergentes, segue que uma seqüência convergente $\left\{g_{n}: n \in \omega\right\}$ em $G_{\mathrm{\kappa}}$ deve ser da forma $\left\{\left(t, w_{n}\right): n \in \omega\right\}$ para algum $t \in\left\langle x_{\xi}: \xi<c\right\rangle$. Suponhamos que $w=\lim _{n \in \omega} w_{n}$. Para cada $n \in \omega$, seja $f(n) \in \operatorname{Fn}(\mathfrak{c}, \mathbb{Z} \backslash\{0\})$ tal que $w_{n}=z_{f(n)}$; passando talvez a uma subseqüência, podemos assumir que $\left\{w_{n}: n \in \omega\right\}$ é injetora e não contém o elemento $0_{\mathbb{T}^{k}}, \operatorname{logo} f: \omega \rightarrow \operatorname{Fn}(\mathfrak{c}, \mathbb{Z} \backslash\{0\})$ é injetora, de modo que podemos tomar $\eta \in P$ tal que $f=f_{\eta}$ e, assim, $z_{\eta}=p_{\eta}-\lim _{n \in \omega} z_{f_{\eta}(n)}=$ $p_{\eta}-\lim _{n \in \omega} z_{f(n)}=p_{\eta}-\lim _{n \in \omega} w_{n}$, mas daí, $z_{\eta}=w$. Note-se que qualquer subseqüência própria de $\left\{w_{n}: n \in \omega\right\}$ está codificada por uma função injetora $g: \omega \rightarrow \mathrm{Fn}(\mathfrak{c}, \mathbb{Z} \backslash\{0\})$ de modo tal que $g=f_{\mu}$ para algum $\mu \in P$. Daí, $z_{\mu}$ também é ponto de acumulação de $\left\{w_{n}: n \in \omega\right\}$, $\log z_{\mu}=z_{\eta}$. Note-se que isto acontece com cada subseqüências distinta de $\left\{w_{n}: n \in \omega\right\}$, obtendo-se desse modo c pontos de acumulação distintos para ela, absurdo. Portanto, $G_{\mathrm{\kappa}}$ é um grupo enumeravelmente compacto e não possui seqüências não triviais convergentes. Naturalmente, $\left|G_{\mathrm{\kappa}}\right|=\mathfrak{c} \mathrm{e}$, como $G_{\mathrm{\kappa}} \subseteq G \times \mathbb{T}^{\kappa} \subseteq \mathbb{T}^{\theta} \times \mathbb{T}^{\mathrm{\kappa}}, w\left(G_{\kappa}\right)=\kappa$.

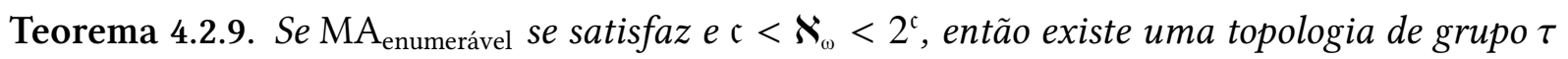
sobre $\mathrm{A}(\mathfrak{c})$ tal que $(\mathrm{A}(\mathfrak{c}), \tau)$ é um grupo de van Douwen de peso $\boldsymbol{\aleph}_{\omega}$.

Demonstração. De acordo com o teorema 1.1.33, temos que a partir de um modelo $\mathcal{M}$ de $\mathrm{ZFC}+\mathrm{GCH}$, obtemos via forcing um modelo de ZFC+(c $\left.=\boldsymbol{\aleph}_{n}\right)+\left(2^{\boldsymbol{\aleph}_{n}}=\boldsymbol{\aleph}_{\omega+1}\right)$ onde $n \in \omega$ é fixo. Note-se que na extensão $\mathcal{M}[\mathbb{G}]$, temos que $\mathfrak{c}=2^{\omega}=\boldsymbol{\aleph}_{n}<\boldsymbol{\aleph}_{\omega}<2^{\mathfrak{c}}=2^{\aleph_{n}}=\boldsymbol{\aleph}_{\omega+1}$, como é requerido. De acordo com a observação 1.1.44, ao acrescentarmos reais de Cohen, $\mathrm{MA}_{\text {enumerável }}$ é satisfeito, logo $\mathcal{M}[\mathbb{G}] \vDash M A_{\text {enumerável. }}$. De acordo com o teorema 2.2.18, em $\mathcal{M}[\mathbb{G}]$ existe uma topologia de grupo $\tau$ sobre $\mathrm{A}(\mathfrak{c})$ de maneira tal que $(\mathrm{A}(\mathfrak{c}), \tau)$ é um grupo de van Douwen. Como $\left(\mathfrak{c}<\boldsymbol{\aleph}_{\omega}<2^{\mathfrak{c}}\right)^{\mathcal{M}[\mathbb{G}]}$, pelo teorema 4.2 .8 em $\mathcal{M}[\mathbb{G}]$ existe uma topologia de grupo $\tau$ sobre $\mathrm{A}(\mathfrak{c})$ munido da qual é um grupo de van Douwen que satisfaz $w(\mathrm{~A}(\mathfrak{c}), \tau)=$ $\aleph_{\omega}$.

Teorema 4.2.10. É consistente com $\mathrm{CH}$ a existência de um grupo Abeliano livre de cardinalidade $2^{\mathfrak{c}}$ e peso $\kappa$ para cada $\kappa \in\left[\mathfrak{c}, 2^{2^{c}}\right]$ sem houver restrições sobre o tamanho de $2^{c}$. Em particular, é consistente que exista um grupo abeliano livre enumeravelmente compacto de peso $\lambda>2^{c}$ onde $\lambda$ é de cofinalidade enumerável. 
Demonstração. No modelo construído no teorema 2.2 .22 , notemos que $\mathrm{CH}$ é válido. Assim, é consistente com $\mathrm{CH}$ a existência de uma topologia enumeravelmente compacta sem sequências não triviais convergentes sobre o grupo abeliano livre $G=\mathrm{A}\left(2^{\mathrm{c}}\right)$. Nessa construção obtemos uma realização de $G$ como subespaço do grupo compacto $\left(\mathbb{T}^{c}\right)^{2^{c}}$. Seguindo um argumento análogo ao encontrado na prova do teorema 4.2.8, obtém-se o resultado. 



\section{CAPÍTULO 5}

\section{Problemas relativos a topologias de grupo enumeravelmente compactas}

\subsection{O problema de Wallace}

Nesta seção consideraremos certas questões relacionadas com semigrupos topológicos, estudando as propriedades que um semigrupo deveria satisfazer para ser um grupo topológico.

\section{Semigrupos de Wallace}

Definição 5.1.1. Um semigrupo $S$ é dito cancelativo se $z x=z y$ implica que $x=y$ e $x z=y z$ implica que $x=y$ para cada $x, y, z \in S$.

As ações à direita e a esquerda $\varrho_{a}$ e $\lambda_{a}$ definidas em um semigrupo $S$ nem sempre são bijeções, como ocorre se consideramos grupos. Mais ainda, num semigrupo, essas ações não são necessariamente injetoras ou sobrejetoras. O anterior explica porque os semigrupos topológicos nem sempre constituem espaços homogêneos, como mostra o seguinte

Exemplo 5.1.2. Consideremos no intervalo fechado $I=[0,1]$ a operação dada por $x * y=$ $\min \{x, y\}$. Naturalmente, $(I, *)$ é um semigrupo topológico que não é um espaço homogêneo; note-se também que as translações não são injetoras.

Facilmente, podemos identificar os semigrupos cancelativos com os semigrupos onde as translações são injetoras (mas não necessariamente sobrejetoras). Assim, o semigrupo considerado no exemplo anterior não é cancelativo.

Nos semigrupos topológicos cancelativos, a compacidade resulta ser uma condição suficiente para se tornarem grupos topológicos, como mostra o seguinte interessante resultado: 
Teorema 5.1.3 (K. Numakura). Todo semigrupo topológico cancelativo Hausdorff compacto $S$ é um grupo topológico.

Demonstração. Primeiro, mostraremos que para cada $a \in S, S a=S$ e $a S=S$, isto é, que cada translação definida sobre $S$ é sobrejetora. Naturalmente, é suficiente mostrar que $a S=S$. Seja $S_{0}=S, S_{1}=a S$, e, indutivamente, para cada $n \in \omega$, seja $S_{n+1}=a S_{n}=a^{n+1} S$. Como $a S \subseteq S$, de acordo com a construção indutiva é claro que para cada $n \in \omega, S_{n+1} \subseteq S_{n}$. Como $S$ é um espaço Hausdorff compacto e as translações são contínuas, os elementos da família $\left\{S_{n}: n \in \omega\right\}$ são subconjuntos fechados, e assim, compactos, de $S$.

Consideremos a seqüência $\left\{a^{n}: n \in \omega\right\} \subseteq S$. Como $S$ é compacto, existe um ponto de acumulação $x \in S$ dessa seqüência; Seja $V$ um aberto em $S$ satisfazendo $x a S \subseteq V$ (pelo menos existe o próprio $S$ ). Pela continuidade da operação e pela compacidade de $a S$, existe uma vizinhança aberta $W$ de $x$ de modo que $W a S \subseteq V$. Seja $k \in \omega$ tal que $a^{k} \in W$. Então, $a^{k} a S \subseteq V$, isto é, $S_{k+1} \subseteq V$. Dado que a família $\left\{S_{n}: n \in \omega\right\}$ é decrescente, temos que para cada $n \geq k+1, S_{n} \subseteq V$.

Seja $b \in S$. Pela continuidade das translações, temos que $x b$ é um ponto de acumulação da seqüência $\left\{a^{n} b: n \in \omega\right\}$. Naturalmente, $a^{n} b \in S_{n} \subseteq V$ para cada $n \geq k+1$, $\operatorname{logo} x b \in \bar{V}$. Daí, tem-se mostrado que $x b$ pertence ao fecho de qualquer vizinhança aberta em $S$ contendo o conjunto $x a S$. Dado que $x a S$ é compacto e $S$ é Hausdorff, $x a S$ é fechado e assim, $x b \in x a S$. Como o semigrupo é cancelativo, $b \in a S$. Sendo $b$ um elemento arbitrário de $S$, conclui-se que $a S=S$; de maneira similar, pode-se provar que $S a=S$.

Seja $a \in S$ arbitrário. Como $S a=S$, existe $e \in S$ tal que $e a=a$. Para cada elemento $y \in S$, dado que $a S=S$, existe $z \in S$ tal que $a z=y$, logo ey $=e a z=a z=y$, ou seja, $e$ é um elemento neutro à esquerda do semigrupo. Como $S$ é cancelativo, o elemento $e$ é único. De maneira similar, existe um elemento neutro à direita $e^{\prime} \in S$ que é único pois $S$ é cancelativo. Assim, $e=e e^{\prime}=e^{\prime}$, de onde $S$ é um monóide. Como $a S=S=S a$ para cada $a \in S$, existe $b \in S$ tal que $a b=e$, e, existe $b^{\prime} \in S$ tal que $b^{\prime} a=e$. Assim, $e a=a=a e=a\left(b^{\prime} a\right)=\left(a b^{\prime}\right) a$; dado que $S$ é cancelativo, $e=a b^{\prime}$, ou seja $a b^{\prime}=a b$, de onde $b^{\prime}=b$. Portanto, $S$ é um grupo. Dado que a operação em $S$ define uma aplicação contínua, $S$ é um grupo paratopológico.

Visto que $S$ é Hausdorff, pela continuidade da operação do grupo, o conjunto $M=$ $\{(a, b) \in S \times S: a b=e\}$ é fechado em $S \times S$. Seja $F \subseteq X$ fechado e tome-se $S_{F}=(S \times F) \cap M$. Observe-se que $F$ e $S \times F$ são compactos e $S_{F}$ é fechado no produto $S \times S$ pois $M$ é fechado; portanto, $S_{F}$ é compacto. Agora, $(a, b) \in S_{F}$ se e somente se $b \in F$ e $a b=e$, ou seja, $a=b^{-1}$. Portanto, $\pi_{1}\left[S_{F}\right]=F^{-1}$. Como $\pi_{1}: S \times S \rightarrow S$ é contínua e $F$ é compacto, $F^{-1}$ é também 
compacto e assim, fechado em $S$. Demonstrou-se então que a aplicação $i: S \rightarrow S$ definida por $i(x)=x^{-1}$ é contínua. Em conseqüência, $S$ é um grupo topológico.

Em 1953 durante o encontro anual da American Mathematical Society realizado em Baltimore (Maryland), depois de notar que distintos autores tinham mostrado que todo semigrupo topológico compacto cancelativo é um grupo topológico, A. D. Wallace salientou que apesar das várias publicações feitas por alguns matemáticos de que essa propriedade também era válida considerando semigrupos que fossem apenas enumeravelmente compactos, "permanecia alguma dúvida sobre a validade dessa afirmação” [71]. A seguinte questão, conhecida como o "problema de Wallace", permaneceu então sem resposta durante mais de quarenta anos:

Questão 5.1 (Problema de Wallace). É todo semigrupo topológico cancelativo e enumeravelmente compacto um grupo topológico?

Definição 5.1.4. Um contraexemplo para a questão 5.1 é dito semigrupo de Wallace.

Em Junho de 1993, durante a Conference on General Topology and its Applications realizada em Slippery Rock, ressurgiu o interesse de vários matemáticos pela questão, entre os que pode-se citar a R.W. Heath e D.L. Grant. A questão, também foi citada por Comfort em $[16,17]$.

Em 1994, Robbie e Svetlichny [53] mostraram o seguinte teorema:

Teorema 5.1.5 (Robbie e Svetlichny). (CH) Existe um semigrupo de Wallace abeliano e completamente regular.

A dependência de $\mathrm{CH}$ no teorema anterior é conseqüência do uso do grupo de Tkačenko [62] (vide 2.2.1) nesse resultado. Contudo, Robbie e Svetlichny basearam a sua construção no seguinte fato mais geral:

Lema 5.1.6. Seja $G$ um grupo topológico abeliano livre de torção, enumeravelmente compacto e sem seqüências não triviais convergentes. Se T é um subsemigrupo infinito enumerável de $G$ que não contém a identidade $e_{G}$, então, cada subconjunto $B \in[T]^{\omega}$ possui um ponto de acumulação $x \in G$ tal que para cada $n \in \omega, x^{n} \notin\left\langle T \cup T^{-1}\right\rangle$ e, além disso, $e_{G} \notin\langle T \cup\{g\}\rangle$.

Demonstração. Vide [53].

Dado que os teoremas 2.2.14 e 2.2.18 implicam a existência de um grupo abeliano livre de van Douwen, aplicando o lema 5.1.6, obtemos também semigrupos de Wallace assumindo $\mathrm{MA}_{\sigma \text {-centrada }}$ e $\mathrm{MA}_{\text {enumerável }}$ respetivamente. De maneira análoga, a construção do teorema 
3.3.11 também implica a existência de semigrupos de Wallace, logo, ainda versões mais fracas de MA não são necessárias para achar contraexemplos à questão de van Douwen.

Teorema 5.1.7. Se existem c ultrafiltros seletivos incomparáveis então existe um semigrupo de Wallace.

Demonstração. De acordo com o teorema 3.3.11 no caso $\kappa=\mathfrak{c},\left\{x_{\xi}: \xi<\mathfrak{c}\right\}$ constitui um conjunto livre de geradores do grupo A(c) (sob homomorfismo topológico). Seja $S=\left\{x_{H}\right.$ : $H \in \operatorname{Fn}(\mathfrak{c}, \mathbb{N}) \backslash\{\varnothing\}\}$; naturalmente, $S$ constitui um semigrupo topológico que não é um grupo, e adicionalmente, pelo fato de ser subsemigrupo do grupo A(c), $S$ é cancelativo.

Seja $\left\{a_{n}: n \in \omega\right\} \subseteq S$ uma seqüência injetora; de acordo com a enumeração $\left\{f_{\xi}: \xi<\mathfrak{c}\right\}$ da família $\mathcal{F}$ pelo lema 3.3.2 existe $\zeta<\mathfrak{c}$ tal que $\left\{x_{f_{\xi}(k)}: k \in \omega\right\}$ é uma subseqüência de $\left\{a_{n}: n \in \omega\right\}$. Pelo teorema 3.3.11, $x_{\zeta}=p_{\zeta}-\lim _{n \in \omega} x_{f_{\zeta}(k)}$, logo, em particular, $x_{\zeta} \in S$ é um ponto de acumulação de $\left\{a_{n}: n \in \omega\right\}$. Em conseqüência, $S$ é enumeravelmente compacto, e assim, constitui um semigrupo de Wallace.

Tomita em [65] mostrou a existência de um semigrupo de Wallace assumindo $\mathrm{MA}_{\text {enumerável }}$ mediante uma técnica diferente, sem que o semigrupo seja subsemigrupo de um grupo abeliano livre, fazendo modificações à construção de Hart e van Mill [40]. Nesse trabalho, provase o seguinte

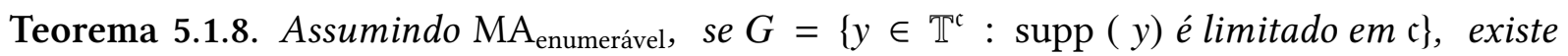
$x \in \mathbb{T}^{c}$ tal que o semigrupo $\{n x+y: n \in \omega$ e y $\in G\}$ é um semigrupo de Wallace.

\section{Alguns comentários sobre independência}

Uma asserção relativamente consistente com ZFC é o Axioma de Kunen:

KA: Existe um ultrafiltro gerado por uma base de filtro de cardinalidade $\omega_{1}$.

Este axioma, foi usado por van Douwen [26] e Hart e van Mill [40] para destacar a aparente dependência de MA das construções feitas por eles. Tomita, em [66], mostrou que sob $\mathrm{KA}+\left(\mathfrak{c}>\omega_{1}\right)$ não existe $x$ como no teorema 5.1.8. Em conexão com esse resultado, a seguinte afirmação mostra que apesar da afirmação estabelecida no teorema 5.1.7, temos também

Teorema 5.1.9. A existência de x como no teorema 5.1.8 é independente da existência de $2^{c}$ ultrafiltros seletivos $\leq_{\mathrm{RK}}$-incomparáveis. 
Demonstração. Sob MA, de acordo com o teorema 5.1.8, temos que existe $x \in \mathbb{T}^{c}$ e existem $2^{c}$ ultrafiltros seletivos. Seja $\mathcal{M}$ o modelo do teorema 3.3.17; como em $\mathcal{M}$ existem $2^{\mathrm{c}}$ ultrafiltros seletivos incomparáveis e cada um deles é gerado por $\aleph_{1}$ conjuntos, $\mathcal{M} \vDash K A+\left(\mathfrak{c}>\omega_{1}\right)$. Em virtude do resultado de Tomita em [66], em $\mathcal{M}$ não existe $x$ satisfazendo essas propriedades. Em conseqüência, é consistente que não existe tal $x$ e existem $2^{c}$ ultrafiltros seletivos.

Teorema 5.1.10. É consistente que existe um semigrupo de Wallace ainda se MA falha totalmente à Baumgartner.

Demonstração. Seja $\mathcal{M}$ um modelo como no teorema 3.3.16. De acordo com o teorema 5.1.7, nesse modelo existe um semigrupo de Wallace e MA falha totalmente à Baumgartner.

\section{Algumas limitações para a existência de semigrupos de Wallace}

Antes do aparecimento do trabalho de Robbie e Svetlichny contendo o primeiro contraexemplo à questão de Wallace, diversos autores tinham estudado as distintas propriedades que um semigrupo de Wallace não poderia ter.

Primeiro, em 1957 Ellis mostrou que um grupo semitopológico (vide [4]) localmente compacto é um grupo topológico; daí, em particular, um semigrupo de Wallace não pode ser um grupo abstrato e localmente compacto.

Mukherjea e Tserpes [52] em 1972 mostraram que qualquer semigrupo topológico cancelativo enumeravelmente compacto satisfazendo o primeiro axioma de enumerabilidade é um grupo topológico. Pfister em 1985, seguindo um trabalho de Brand, mostrou que num semigrupo topológico de Tychonoff enumeravelmente compacto que é algebricamente um grupo, a inversão é contínua, o que faz dele um grupo topológico. Como conseqüência desses resultados, um semigrupo de Wallace não pode ser 1-enumerável nem pode ser completamente regular e um grupo abstrato simultaneamente.

A respeito de espaços completamente regulares, D.L. Grant mostrou em 1993 que um semigrupo cancelativo seqüencialmente compacto constitui um grupo algébrico; em conjunção com o resultado obtido por Pfister, a asserção de Grant implica que um semigrupo com essas caraterísticas, é também um grupo topológico, ou seja, também não existem semigrupos de Wallace sendo completamente regulares e seqüencialmente compactos simultaneamente. De igual maneira, Grant mencionou que não existem semigrupos de Wallace $\omega$-limitados.

De acordo com as relações de implicação entre as propriedades relacionadas com a compacidade, parece natural perguntar se também não existem semigrupos de Wallace completa- 
mente regulares e $p$-compactos, sendo $p \in \omega^{*}$. No trabalho [65], Tomita responde de maneira afirmativa essa questão:

Teorema 5.1.11. Seja $p \in \omega^{*}$. Todo semigrupo topológico cancelativo p-compacto é um grupo topológico.

Demonstração. Vide [65] 3.1.

\subsection{Uma questão de Dikranjan e Shakhmatov}

Em [22], D. Dikranjan e D. Shakhmatov mostraram que os grupos livres não abelianos não admitem topologias de grupo enumeravelmente compactas. A este respeito, surge a seguinte questão (vide [18]):

Questão 5.2. Para quais cardinais $\kappa$ existe uma topologia de grupo enumeravelmente compacta sobre $\mathrm{A}(\kappa)$ ?

De acordo com o teorema 2.2.18, Koszmider et al. exibiram um modelo de forcing no qual $\mathrm{CH}$ é válido e $2^{\mathrm{c}}$ constitui um de tais cardinais. Como vimos, em particular é consistente que para cada cardinal infinito satisfazendo $\kappa=\kappa^{\omega} \leq 2^{\mathrm{c}}$ existe uma topologia de grupo enumeravelmente compacta sobre $A(\kappa)$. Escolhendo um modelo que adicionalmente satisfaça $\boldsymbol{\aleph}_{\omega}>2^{\mathrm{c}}$, cada cardinal $\kappa \in\left[\mathfrak{c}, 2^{\mathfrak{c}}\right]$ é regular e não enumerável, ou seja, satisfaz $\kappa^{\omega}=\kappa$, implicando isto que o grupo $\mathrm{A}(\kappa)$ pode ser munido de uma topologia de grupo enumeravelmente compacta.

Mas, qual é a situação se $2^{\mathfrak{c}}>\boldsymbol{\aleph}_{\omega}$ ? Como vimos no teorema 4.2.6, é consistente com ZFC a existência de um grupo enumeravelmente compacto cuja cofinalidade seja enumerável. No trabalho publicado em 2004 [15], Castro-Pereira e Tomita modificaram as construções feitas em [46] e [67] para obter um modelo no qual para cada $\kappa \in\left[\mathfrak{c}, 2^{\mathfrak{c}}\right]$ existe uma topologia de grupo enumeravelmente compacta sobre $A(\kappa)$ sem impor restrições ao tamanho de $2^{\mathfrak{c}}$; nesse modelo, $2^{c}$ inclusive pode ser maior que infinitos cardinais de cofinalidade enumerável.

Em [23], Dikranjan e Shakhmatov usaram também uma noção de forcing enumeravelmente fechada, para obter a partir de um modelo de $\mathrm{CH}$ uma classificação dos grupos abelianos de cardinalidade menor ou igual a $2^{c}$ que admitem uma topologia de grupo enumeravelmente compacta.

A extensão de um modelo que satisfaz $\mathrm{CH}$ obtida através de uma noção de forcing enumeravelmente fechada satisfaz também $\mathrm{CH}$, de modo que todas as topologias de grupo enumeravelmente compactas obtidas sobre o grupo $A\left(2^{c}\right)$ descritas anteriormente são construídas via 
forcing tendo como base um modelo que satisfaz $\mathrm{CH}$. Como vimos no teorema 3.3.11, assumindo a existência de $2^{\mathrm{c}}$ ultrafiltros seletivos $\leq_{\mathrm{RK}}$-incomparáveis, é possível também construir uma topologia de grupo enumeravelmente compacta sobre $A\left(2^{c}\right)$, de modo que para uma tal construção não são necessários forcing ou $\mathrm{CH}$.

$\mathrm{Na}$ construção apresentada na seção 3.3., cada ultrafiltro seletivo foi usado para uma única seqüência. A construção de uma topologia de grupo enumeravelmente compacta sobre o grupo abeliano livre de posto estritamente maior do que $2^{c}$, requer necessariamente que algum ultrafiltro testemunhe a existência de pontos de acumulação para uma grande quantidade de seqüências dado que a cardinalidade do espaço $\beta \omega$ todo é $2^{\mathfrak{c}}$. Como assinalam MadariagaGarcia e Tomita em [50], a chave para responder à questão de Dikranjan e Shakhmatov de maneira afirmativa para qualquer cardinal $\kappa$ satisfazendo $\kappa=\kappa^{\omega}$, provavelmente é a solução da questão a seguir:

Questão 5.3. É consistente com ZFC a existência de uma topologia de grupo enumeravelmente compacta sobre o grupo abeliano livre de cardinalidade $2^{2^{c}}$ ?

\subsection{Sobre topologias de grupo independentes}

\section{A noção de complementaridade de topologias}

O estudo do reticulado $\mathscr{T}(X)$ de todas as topologias definidas sobre um conjunto $X$ foi iniciado por Birkhoff [11] nos anos trinta. O fato de que $\mathscr{T}(X)$ é um reticulado complementado foi estabelecido, de maneira independente, em 1966 por A. Steiner [59] e van Rooji [54].

Bagley [5] e E. Steiner [61] estudaram o reticulado $\mathscr{T}_{1}(X)$, formado pelas topologias $T_{1}$ que podem ser definidas sobre um conjunto $X$ e os $T_{1}$-complementos.

De maneira geral, duas topologias distintas $\tau_{1}, \tau_{2} \in \mathscr{T}(X)$ são ditas complementares se $\tau_{1} \cup \tau_{2}$ gera a topologia discreta e $\tau_{1} \cap \tau_{2}$ é a topologia caótica sobre $X$. No reticulado $\mathscr{T}_{1}(X)$, temos uma definição análoga:

Definição 5.3.1. Seja $X$ um conjunto não vazio. Duas topologias não discretas $\tau_{1}, \tau_{2} \in \mathscr{T}_{1}(X)$, são ditas $T_{1}$-complementares se $\tau_{1} \cup \tau_{2}$ gera a topologia discreta e $\tau_{1} \cap \tau_{2}$ é a topologia cofinita sobre $X$.

A. Steiner e E. Steiner [60] mostraram que a topologia usual definida sobre um subconjunto denso enumerável de $\mathbb{R}$ possui um $T_{1}$-complementar. Isto implica, junto com os resultados obtidos anteriormente, que, de fato, a topologia usual sobre $\mathbb{R}$ possui um $T_{1}$-complementar. 
B. Anderson e D. Stewart [3] estenderam alguns resultados dos trabalhos citados para obter o seguinte teorema:

se um espaço $T_{1}$ tem um subespaço denso enumerável que é metrizavel, então possui um $T_{1}$-complementar.

Pouco tempo depois, B.A. Anderson [1, 2] estudou famílias de topologias mutuamente complementares e provou que todo espaço Hausdorff localmente compacto ou de Fréchet possui um $T_{1}$-complementar.

D. Dikranjan et al.[25], provaram que a situação resulta ser diferente no reticulado $\mathscr{L}(G)$ de todas as topologias de grupo $T_{2}$ definidas sobre um grupo abstrato $G$. Nesse trabalho mostra-se que nenhuma topologia $\tau \in \mathscr{L}(G)$ sobre um grupo infinito admite uma topologia $T_{1}$-complementar. Para provar tal afirmação, precisamos alguns resultados simples.

Lema 5.3.2. Se $G$ um grupo e $\tau_{1}$ e $\tau_{2} \in \mathscr{L}(G)$, então $\wp(G)=\left\langle\tau_{1} \cup \tau_{2}\right\rangle$ se e somente se existem $U \in \tau_{1}, W \in \tau_{2}$ tais que $U \cap W=\left\{e_{G}\right\}$.

Demonstração. Note que se $\tau_{1}$ e $\tau_{2}$ geram a topologia discreta, dadas duas vizinhanças de $e_{G}$, $V \in \tau_{1}, W \in \tau_{2}, V \cap W=\left\{e_{G}\right\}$. De outra parte, note que se existem $V \in \tau_{1}, W \in \tau_{2}$ tais que $V \cap W=\left\{e_{G}\right\}$ temos que para cada $x \in G,\{x\}=x\left\{e_{G}\right\}$ é aberto na topologia $\left\langle\tau_{1} \cup \tau_{2}\right\rangle$, ou seja, $\tau_{1} \cup \tau_{2}$ gera a topologia discreta.

Definição 5.3.3. Seja $G$ um grupo topológico; se $U$ é uma vizinhança da identidade $e_{G}$, dizemos que $K \subseteq G$ é uniformemente $U$-discreto à esquerda se para qualquer $\{x, y\} \in[K]^{2}$, temos que $x U \cap y U=\varnothing$.

Lema 5.3.4. Seja $G$ um grupo topológico. Se $K \subseteq G$ é uniformemente $U$-discreto à esquerda para alguma vizinhança $U$ da identidade $e_{G}$, então $K$ é fechado e discreto em $G$.

Demonstração. Seja $V$ uma vizinhança simétrica de $e_{G}$ em $G$, isto é, uma vizinhança satisfazendo $V=V^{-1}$, de modo que $V^{2} \subseteq U$. Afirmamos que para cada $x \in G,|x V \cap K| \leq 1$. Com efeito, se $y, z \in x V \cap K$, então $z \in y V^{2} \subseteq y U$; de acordo com a hipótese, $z=y$. Portanto, $|x V \cap K| \leq 1$ e assim, $K$ é fechado e discreto em $G$.

Proposição 5.3.5. Seja $G$ um grupo com identidade $e_{G}$. Se $\tau_{1}, \tau_{2} \in \mathscr{L}(G)$ são tais que $\wp(G)=$ $\left\langle\tau_{1} \cup \tau_{2}\right\rangle$ então existem $U \in \tau_{1}, V \in \tau_{2}$ tais que $U \cap W=\{e\}$, onde $U$ é uniformemente $W$-discreto à esquerda e $W$ é uniformemente $U$-discreto à esquerda. 
Demonstração. Pelo lema 5.3.2, existem $U_{0} \in \tau_{1}, W_{0} \in \tau_{2}$ tais que $U_{0} \cap W_{0}=\{e\}$. Sejam $U \in \tau_{1}$, $V \in \tau_{2}$ vizinhanças simétricas da identidade $e_{G}$ de modo que $U^{2} \subseteq U_{0}$ e $W^{2} \subseteq W_{0}$; de acordo com a construção, note-se que para cada $\{x, y\} \in[W]^{2}$, temos que $x U \cap y W=\varnothing$ e para cada $\{a, b\} \in[U]^{2}, a W \cap b W=\varnothing$.

Teorema 5.3.6. Nenhum grupo $G$ com mais de um elemento admite topologias de grupo $T_{1}$ complementares, isto é, nenhuma topologia sobre $G$ admite um $T_{1}$-complementar em $\mathscr{L}(G)$.

Demonstração. Como toda topologia $T_{1}$ definida sobre um conjunto finito é discreta, é suficiente considerar o caso em que $|G| \geq \omega$. Suponhamos que $\tau_{1}$ e $\tau_{2} \in \mathscr{L}(G)$ geram a topologia discreta; vamos mostrar que $\tau_{1} \cap \tau_{2}$ não é a topologia dos cofinitos sobre $G$.

Pela proposição 5.3.5, existem vizinhanças da identidade $e_{G}, U \in \tau_{1}, W \in \tau_{2}$ tais que $U \cap W=\{e\}$ e $W$ é uniformemente $U$-discreta à esquerda. Seja $W_{1} \in \tau_{2}$ uma vizinhança aberta de $e_{G}$ tal que $W_{1}^{2} \subseteq W$; se $F={\overline{W_{1}}}^{\tau_{2}}$, naturalmente $F$ é um subconjunto infinito fechado na topologia $\tau_{2}$ e $F \subseteq W \neq G$. De outra parte, o lema 5.3.4 implica que $W$ é um subconjunto fechado e discreto na topologia $\tau_{1}$; em conclusão, temos que $F$ é um subconjunto infinito próprio e fechado nas topologias $\tau_{1}$ e $\tau_{2}$, isto é, $\tau_{1} \cap \tau_{2}$ não é a topologia dos conjuntos cofinitos de $G$, daí, $\tau_{1}$ e $\tau_{2}$ não são $T_{1}$-complementares.

No caso dos grupos abelianos, temos o seguinte resultado:

Teorema 5.3.7. Seja $G$ um grupo abeliano. Se $\tau_{1}, \tau_{2} \in \mathscr{L}(G)$ são tais que $\wp(G)=\left\langle\tau_{1} \cup \tau_{2}\right\rangle$, então a maior topologia $\tau \in \mathscr{L}(G)$ tal que $\tau \subseteq \tau_{1} \cap \tau_{2}$ é de Hausdorff. Em conseqüência, nenhum grupo abeliano admite duas topologias de grupo $T_{1}$-complementares.

Demonstração. Sejam $\tau_{1}, \tau_{2} \in \mathscr{L}(G)$. Notemos que se $\tau=\left\langle\tau_{1} \cup \tau_{2}\right\rangle$, o subgrupo $\Delta_{G}=\{(x,-x)$ : $x \in G\}$ considerado como subespaço de $H=\left(G, \tau_{1}\right) \times\left(G, \tau_{2}\right)$ é homeomorfo a $(G, \tau)$. Notemos também que o epimorfismo $f: G \times G \rightarrow G$ definido por $(x, y) \mapsto x+y$ satisfaz $\operatorname{ker}(f)=\Delta_{G}$; daí, $G \times G / \Delta_{G} \cong G$ considerado como espaço quociente pela aplicação $f$, encontra-se munido da topologia $\tau_{1} \wedge \tau_{2}$ dado que para cada $U \in \tau_{1}, V \in \tau_{2}, f(U \times V)=U+V$.

Se $\wp(G)=\left\langle\tau_{1} \cup \tau_{2}\right\rangle$, então, $\Delta_{G}$ é um subgrupo discreto e fechado de $H$; daí, $\left(G, \tau_{1} \wedge \tau_{2}\right) \cong$ $H / \Delta_{G}$ é de Hausdorff, de onde as topologias $\tau_{1}$ e $\tau_{2}$ não podem ser complementares.

O resultado anterior torna especialmente natural a bifurcação da noção de $T_{1}$-complementaridade em transversalidade e $T_{1}$-independência quando se trata de grupos topológicos.

Definição 5.3.8. Seja $X$ um conjunto. Duas topologias não discretas $\tau_{1}$ e $\tau_{2} \in \mathscr{T}_{1}(X)$ são chamadas de transversais se $\tau_{1} \cup \tau_{2}$ gera a topologia discreta sobre $X$. Por sua vez, $\tau_{1}$ e $\tau_{2} \in \mathscr{T}_{1}(X)$ são ditas $T_{1}$-independentes se $\tau_{1} \cap \tau_{2}$ é a topologia cofinita sobre $X$. 


\section{Independência em topologias de grupo}

Existe uma diferença crucial entre a transversalidade e a $T_{1}$-independência quando se trata de grupos topológicos. De fato, a união $\tau_{1} \cup \tau_{2}$ de duas topologias de grupo sobre $G$ sempre gera uma topologia de grupo, porém a interseção $\tau_{1} \cap \tau_{2}$ não necessariamente constitui uma topologia de grupo. Os principais resultados do trabalho [25] concernem a grupos que admitem topologias de grupo transversais; inclui-se nessa classe muitos (mas nem todos) os grupos localmente compactos tais como o grupo aditivo $(\mathbb{R},+)$, o grupo multiplicativo $\left(\mathbb{C}^{*}, \times\right)$, os grupos lineares $\mathrm{GL}(n, \mathbb{R})$ e $\mathrm{GL}(n, \mathbb{C})$, etc. Nesta seção, estudaremos as construções feitas por Tkačenko e Yasčenko de exemplos de topologias de grupo $T_{1}$-independentes: sob MA, mostra-se que as topologias usuais definida sobre os grupos $\mathbb{R}$ e $\mathbb{T}$ admitem uma topologia de grupo $T_{1}$-independente, cada uma das quais é, necessariamente, uma topologia enumeravelmente compacta e sem seqüências não triviais convergentes.

O estudo de topologias de grupo $T_{1}$-independentes, envolve dois tópicos que aparentemente não tem relação nenhuma: existência de subconjuntos incondicionalmente fechados em grupos topológicos e existência de topologias de grupo enumeravelmente compactas sem seqüências não triviais convergentes.

Definição 5.3.9. Seja $G$ um grupo abstrato. $A \subseteq G$ é dito incondicionalmente fechado em $G$ se $A$ é fechado em $(G, \tau)$ para qualquer $\tau \in \mathscr{L}(G)$.

Proposição 5.3.10. Um grupo G que possui um subconjunto próprio infinito que é incondicionalmente fechado, não admite duas topologias de grupo $T_{1}$-independentes.

Demonstração. Seja $A \subsetneq G$ um subconjunto infinito e incondicionalmente fechado; suponhamos por absurdo que existem topologias $\tau_{1}, \tau_{2} \in \mathscr{L}(G)$ que são $T_{1}$-independentes. Dado que $A$ é fechado nas duas topologias, $G \backslash A \in \tau_{1}$ e $G \backslash A \in \tau_{2}$, ou seja, $G \backslash A$ é aberto em $\tau_{1} \cap \tau_{2}=\tau_{\operatorname{cof}(G)}$; portanto, $A \in[G]^{<\omega} \backslash\{\varnothing\}$, uma contradição.

Notemos que a proposição anterior brinda uma condição necessária para topologias de grupo que admitam topologias independentes. Mais uma condição necessária é que os grupos que admitem topologias $T_{1}$-independentes não são enumeráveis.

Teorema 5.3.11. Um grupo infinito enumerável não admite topologias de grupo $T_{1}$-independentes.

Demonstração. Seja $G$ um grupo infinito enumerável. Suponhamos por absurdo que existem topologias $\sigma_{1}, \sigma_{2} \in \mathscr{L}(G) T_{1}$-independentes. Por um resultado de Arkhangel'skiǐ, para cada $i=1,2$, existe $\tau_{i} \in \mathscr{L}(G)$ onde $\tau_{i} \subseteq \sigma_{i}$ e $w\left(G, \tau_{i}\right)=\omega$. Naturalmente, as topologias $\tau_{1}$ e $\tau_{2}$ 
também são $T_{1}$-independentes; além disso, dado que duas topologias de grupo não podem ser $T_{1}$-complementares, $\wp(G) \neq \tau=\left\langle\tau_{1} \cup \tau_{2}\right\rangle$. De acordo com a escolha das topologias, $w(G, \tau)=$ $\omega$; portanto, quando consideramos a topologia $\tau$, existe uma seqüência injetora $\left\{x_{n}: n \in\right.$ $\omega\} \subseteq G$ que converge ao elemento identidade $e_{G}$. Em conseqüência, $E=\left\{e_{G}\right\} \cup\left\{x_{n}: n \in \omega\right\}$ é um subconjunto fechado infinito quando consideramos as topologias $\tau_{1}$ e $\tau_{2}$. Se $G \backslash E$ for finito e distinto de vazio, teríamos um aberto finito em $\tau$, o que contradiz $\tau \neq \wp(G)$, logo $G \backslash E$ deve ser infinito mas isso também é impossível posto que $G \backslash E$ é aberto em $\tau_{1} \cap \tau_{2}=\tau_{\text {cof }}$.

A situação torna-se diferente no caso dos grupos não enumeráveis, mas, precisamos de axiomas adicionais a ZFC. De fato, vai se mostrar que sob MA, cada um dos grupos $\mathbb{R}, \mathbb{T}, \mathbb{C}$ admite uma topologia de grupo de Hausdorff independente da topologia euclidiana usualmente considerada em cada um deles. Qualquer construção de uma topologia de grupo independente sobre esses grupos resulta ser o bastante complicada a causa da seguinte observação:

Proposição 5.3.12. Seja $G$ um grupo. Se $\tau, \sigma \in \mathscr{L}(G)$ são topologias $T_{1}$-independentes e $\tau$ é seqüencial, então o espaço $(G, \sigma)$ é enumeravelmente compacto e sem seqüências não triviais convergentes.

Demonstração. Vamos mostrar que $(G, \sigma)$ é enumeravelmente compacto. Suponha por absurdo que existe $A \in[G]^{\omega}$ fechado e discreto em $(G, \sigma)$. Como $\sigma$ não é a topologia discreta, temos que $|G \backslash A| \leq \omega$, logo, pela independência assumida entre as topologias $\sigma$ e $\tau$, $A$ não pode ser fechado em $(G, \tau)$. Como $(G, \tau)$ é seqüencial, existe uma seqüência injetora $\left\{x_{a}: a \in \omega\right\} \subseteq A$ que convergindo a um ponto $a \in G \backslash A$. Daí, o conjunto $B=\left\{x_{a}: a \in \omega\right\} \cup\{a\}$ é fechado em $(G, \sigma)$ e em $(G, \tau)$, mas daí $G \backslash B \in \tau \cap \sigma$, absurdo pois $B$ é infinito. Daí, $(G, \sigma)$ é enumeravelmente compacto.

Agora, tomemos $\left\{x_{n}: n \in \omega\right\} \subseteq(G, \sigma)$ uma seqüência injetora e suponhamos por absurdo que converge a um ponto $x \in G$ nessa topologia. Seja $J \in[\omega]^{\omega}$ um subconjunto cujo complementar é infinito e consideremos a subseqüência $\left\{x_{j}: j \in J\right\}$. Como $G \backslash\left\{x_{j}: j \in J\right\}$ é infinito, pela $T_{1}$-independência entre $\sigma$ e $\tau,\left\{x_{j}: j \in J\right\} \cup\{x\}$ não pode ser fechado em $(G, \tau)$ de modo que existe $I \in[J]^{\omega}$ tal que a seqüência $\left\{x_{j}: j \in I\right\}$ converge a um ponto $x^{\prime} \in G \backslash\{x\}$ considerando a topologia $\tau$ em $G$. Daí, $F=\left\{x_{j}: j \in I\right\} \cup\left\{x, x^{\prime}\right\}$ é fechado em $(G, \tau)$ mas também é fechado em $(G, \sigma)$ pois $\left\{x_{j}: j \in I\right\}$ converge a $x$ quando consideramos essa topologia. Em conseqüência, $G \backslash F \in \tau \cap \sigma$, absurdo pois $F \neq G$ é infinito.

Um fato bem conhecido é que no grupo abstrato $\mathbb{R}$ existe uma topologia de grupo $\tau_{K}$ tal que $\left(\mathbb{R}, \tau_{K}\right)$ é um espaço Hausdorff compacto. Como todo grupo topológico infinito e compacto 
é diádico, e todo espaço diádico possui seqüências não triviais convergentes, em virtude da proposição anterior, $\tau_{K}$ não pode ser $T_{1}$-independente da topologia usual $\tau$ considerada em $\mathbb{R}$. De maneira diferente, usando MA pode-se construir uma topologia enumeravelmente compacta sobre $\mathbb{R}$ que é $T_{1}$-independente da topologia usual $\tau$. Mais ainda, esta topologia pode ser escolhida de modo tal que seja conexa e localmente conexa.

O estudo das topologias de grupo independentes tem ainda outro ingrediente. A existência de uma topologia de grupo compacta sobre um grupo abeliano abstrato implica restrições fortes sobre a estrutura algébrica dele. O estudo das restrições para a cardinalidade de um grupo que admite uma topologia de grupo pseudocompacta foi iniciada por van Douwen. A mais simples delas estabelece que a cardinalidade de um grupo pseudocompacto infinito é pelo menos c. Depois, Comfort e Robertson provaram que todo grupo abeliano de torção que é pseudocompacto é de ordem finita. Este trabalho foi continuado por Dikranjan e Shakhmatov, achando várias restrições de tipo algébrico para grupos abelianos pseudocompactos.

Em geral, a existência de uma topologia de grupos pseudocompacta definida sobre um grupo $G$, depende de axiomas adicionais a ZFC, exceto no caso $|G|=\mathfrak{c}$. De fato, de acordo com o trabalho de Dikranjan e Shakhmatov [22], um grupo abeliano $G$ de cardinalidade $\mathfrak{c}$ admite uma topologia de grupo pseudocompacta se e somente se $|G / \operatorname{tor}(G)|=\mathfrak{c}$ ou $\operatorname{or}(G)=n$ e para cada $m \mid n$ o conjunto $m G=\{m x: x \in G\}$ é finito ou tem cardinalidade c. Portanto, é natural considerar inicialmente grupos abelianos satisfazendo o segundo axioma de enumerabilidade que admitam topologias de grupo independentes.

Note-se que se $G$ é um grupo que admite uma topologia $\tau$ de grupo compatível de peso $\omega$, o espaço $(G, \tau)$ é seqüencial; assim, como em virtude do teorema 5.3.5, qualquer topologia $\sigma \in \mathscr{L}(G)$ independente de $\tau$ é enumeravelmente compacta, é natural prever que as restrições algébricas e de cardinalidade sobre $G$ são ainda mais fortes. Duas delas já foram mencionadas: todos os subconjuntos próprios incondicionalmente fechados num grupo tal são finitos e, esse grupo, se é infinito, deve ser necessariamente de cardinalidade c. Em principio, sobre MA, não existem mais restrições. De fato, podemos dar uma caracterização algébrica completa da primeira das condições:

Definição 5.3.13. Um grupo abeliano $G$ é quase livre de torção se para cada $n \in \omega$, o subgrupo $G[n]=\{x \in G: n x=0\}$ é finito.

Exemplo 5.3.14. $\quad$ i. O grupo $\mathbb{T}$ é quase livre de torção. Note que para cada $n \in \mathbb{N}, \mathbb{T}[n]=$ $\left\{x \in \mid T: x^{n}=1\right\}=\left\{e^{\frac{2 \pi i m}{n}}: 0 \leq m \leq n-1\right\}$, isto é, $\mathbb{T}[n]$ é o conjunto das raízes $n$-ésimas da unidade. 
ii. Existem grupos de torção infinitos que são quase livres de torção. Com efeito, se $p \in \mathbb{N}$ é um número primo, definimos $R=\left\{\frac{q}{p^{k}}: q \in \mathbb{Z}, k \in \mathbb{N}\right\}$; o grupo quociente $R / \mathbb{Z}:=\mathbb{Z}_{p^{\infty}}$ é chamado de grupo quase-cíclico. Notemos que $\mathbb{Z}_{p^{\infty}}$ é quase livre de torção. De fato, seja $n \in \mathbb{N} ;$ se $\operatorname{mdc}\left(q, p^{k}\right)=1$ então $\left[\frac{q}{p^{k}}\right] \in \mathbb{Z}_{p^{\infty}}[n]$ se e somente se $\left[n \frac{q}{p^{k}}\right]=[0]$ se e somente se $n \frac{q}{p^{k}} \in \mathbb{Z}$ se e somente se $p^{k} \mid n$.

iii. O subgrupo de torção infinito $\mathbb{Q} / \mathbb{Z} \cong[0,1] \cap \mathbb{Q} \subseteq \mathbb{T}$ é quase livre de torção.

Obviamente, o subgrupo de torção de um grupo abeliano é sempre enumerável.

Para mostrarmos o resultado principal, precisamos de alguns lemas. O primeiro deles, dá a idéia básica da construção apresentada nesta seção.

Lema 5.3.15. Sejam $K$ um grupo topológico compacto e $(G, \tau)$ um grupo topológico tal que $|G|=$ c. Suponhamos que existe um monomorfismo $h: G \rightarrow K^{\mathrm{c}}$ satisfazendo:

i. $h(G)$ é um subgrupo de $K^{\mathrm{c}}$ enumeravelmente compacto e sem seqüências não triviais convergentes, $e$,

ii. para cada subconjunto fechado $F$ de $(G, \tau)$ de cardinalidade $\mathfrak{c}, h(F)$ é denso em $K^{\mathfrak{c}}$.

Se o é a topologia inicial associada a $h$, isto é, $\sigma=\left\{h^{-1}[U \cap h(G)]: U\right.$ é aberto em $\left.K^{c}\right\}$, então as topologias $\tau$ e $\sigma$ são $T_{1}$-independentes.

Demonstração. Seja $V \in \tau$ não vazio. Dado que $h$ é injetora, temos que $\sigma$ é uma topologia $T_{2}$, daí, para mostrar que $\tau$ e $\sigma$ são $T_{1}$-independentes é suficiente mostrar que um subconjunto fechado nas duas topologias deve ser finito. Notemos também que como $h$ é monomorfismo, $h(G \backslash V)=h(G) \backslash h(V)$.

Se $|G \backslash V|=\mathfrak{c}$, de acordo com a hipótese $h(G \backslash V)=h(G) \backslash h(V)$ é denso em $h(G)$, logo $h(V)$ não é aberto em $h(G)$; daí, $h^{-1}[h(V)]=V \notin \sigma$.

Se $\omega \leq|G \backslash V|<\mathfrak{c}$ e $h(G \backslash V)$ for fechado em $h(G)$, de acordo com a condição i), temos que $h(G \backslash V)$ é enumeravelmente compacto; de outra parte, como a cardinalidade este conjunto é menor do que $\mathfrak{c}, h(G \backslash V)$ é disperso. Da regularidade de $K^{\mathfrak{c}}$, segue também que $h(G \backslash V)$ é regular; agora, todo espaço infinito, regular, enumeravelmente compacto e disperso possui uma seqüência não trivial convergente, o que contradiz a condição i). Conclui-se então que $h(V)=h(G) \backslash h(G \backslash V)$ não é aberto em $h(G)$, logo, $V \notin \sigma$.

Dado que $|G|=\mathfrak{c}$, se $V \in \sigma \cap \tau$ necessariamente $|G \backslash V|<\omega$, ou seja, $\tau$ e $\sigma$ são independentes. 
Para construir um monomorfismo adequado satisfazendo as condições i) e ii) do lema precedente, é preciso contarmos com alguns resultados auxiliares. O primeiro deles, que constitui o núcleo da construção principal desta seção, faz uso do grupo dual de Pontryagin $\hat{G}$ de um grupo abeliano $G$.

Definição 5.3.16. Se $G$ é um grupo topológico abeliano, então um homomorfismo contínuo $\gamma$ : $G \rightarrow \mathbb{T}$ é dito um caráter. O conjunto $\hat{G}$ de todos os caracteres definidos em $G$ é denominado grupo dual de Pontryagin.

É fácil ver que $\hat{G}$ é um subgrupo fechado do grupo produto $\mathbb{T}^{G}$, logo $\hat{G}$ é também compacto.

Lema 5.3.17. Se $S$ é um subconjunto infinito de um grupo abeliano $G$ tal que para cada $x \in G e$ $n \in \mathbb{N}$, o conjunto $S \cap(x+G[n])$ é finito, então o conjunto $H_{S}=\{h \in \hat{G}: h(S)$ é denso em $\mathbb{T}\} e ́$ um $G_{\delta}$ formado por subconjuntos abertos densos em $\hat{G}$.

Demonstração. Seja $\left\{s_{n}: n \in \omega\right\}$ um subconjunto denso enumerável de $\mathbb{T}$. Para cada $n \in \omega$, consideremos o conjunto

$$
U_{n}=\left\{h \in \hat{G}: \text { existe } x \in S \text { tal que }\left|h(x) \backslash S_{n}\right|<\frac{1}{n+1}\right\}
$$

Afirmamos que $U_{n}$ é aberto em $\hat{G}$; com efeito, se $f \in U_{n}$, então existe $x \in S$ tal que $\left|f(x)-S_{n}\right|<$ $\frac{1}{n+1}$, $\operatorname{logo}$ podemos tomar $\varepsilon>0$ de modo que $\left|f(x)-s_{n}\right|+\varepsilon<\frac{1}{n+1}$. Se consideramos a vizinhança básica de $f, W(f ; x, \varepsilon)=\{g \in \hat{G}:|f(x) \backslash g(x)|<\varepsilon\}$, temos que para todo $g \in W(f ; x, \varepsilon)$

$$
\begin{aligned}
\left|g(x)-s_{n}\right| & \leq|g(x)-f(x)|+\left|f(x)-s_{n}\right| \\
& <\left|f(x)-s_{n}\right|+\varepsilon<\frac{1}{n+1}
\end{aligned}
$$

isto é, $g \in U_{n}$. Daí, $W(f ; x, \varepsilon) \subseteq U_{n}$,ou seja, $U_{n}$ é aberto.

Vamos mostrar agora que $H_{S}=\bigcap_{n \in \omega} U_{n}$ e que cada $U_{n}$ é denso em $\hat{G}$.

Seja $h \in \bigcap_{n \in \omega} U_{n}$ e seja $V \subseteq \mathbb{T}$ um aberto não vazio. Tomemos $k \in \omega$ tal que $s_{k} \in V$ e o arco $I\left(s_{k}, \frac{1}{k+1}\right)=\left\{y \in \mathbb{T}:\left|y \backslash s_{k}\right|<\frac{1}{k+1}\right\}$ esteja totalmente contido em $V$. Dado que $h \in U_{k}$, existe $x \in S$ tal que $\left|h(x)-s_{k}\right|<\frac{1}{k+1}$; daí, $h(s) \in I\left(s_{k}, \frac{1}{k+1}\right) \subseteq V$ e assim, $h(S) \cap V \neq \varnothing$. Em conseqüência, $h(S)$ é denso em $\mathbb{T}$, isto é, $h \in H_{S}$. Portanto, $\bigcap_{n \in \omega} U_{n} \subseteq H_{S}$.

De outra parte, se $h \in H_{S}$ e $k \in \omega$ temos que $h(S) \cap I\left(s_{k}, \frac{1}{k+1}\right) \neq \varnothing$, de modo que existe $x \in S$ tal que $\left|h(x)-s_{k}\right| \leq \frac{1}{k+1}$, isto é, $h \in U_{k}$. Dado que isto ocorre para todo $k \in \omega, h \in \bigcap_{n \in \omega} U_{n}$, ou seja, $H_{S} \subseteq \bigcap_{n \in \omega} U_{n}$. 
Basta mostrar agora que cada $U_{n}$ é denso em $\hat{G}$. Sejam $f \in \hat{G}, z_{1}, \ldots, z_{r} \in G$ e $\varepsilon>0$. Consideremos o aberto básico $W_{0}=W\left(f ; z_{1}, \ldots, z_{r}, \varepsilon\right) \subseteq \hat{G}$. Vamos mostrar que $W_{0} \cap U_{n} \neq \varnothing$.

Seja $H=\left\langle z_{1}, \ldots, z_{r}\right\rangle$. Como $H$ é um grupo abeliano finitamente gerado, é soma direta de subgrupos cíclicos, logo existem $y_{1}, \ldots, y_{m} \in H$ tais que $H=\bigoplus_{i=1}^{m}\left\langle y_{i}\right\rangle$. Como o conjunto $\left\{y_{1}, \ldots, y_{n}\right\}$ é linearmente independente, temos que $m \leq r$; adicionalmente, como os elementos $z_{1}, \ldots, z_{r}$ são combinações lineares de $y_{1}, \ldots y_{m}$, pela continuidade da operação do grupo, claramente podemos achar $\delta>0$ tal que $W_{1}=W\left(f ; y_{1}, \ldots y_{m}, \delta\right) \subseteq W_{0}$, de onde resulta suficiente mostrar que $U_{n} \cap W_{1} \neq \varnothing$.

Para cada $x \in S$, tomemos $d(x)=\min \{k \in \omega: k x \in H, k \neq 0 \in G\}$. Se $k x \notin H$ para todo $k \in \omega \backslash\{0\}$, definimos $d(x)=\infty$. Consideremos os seguintes casos:

A. Existe $x \in S$ tal que $d(x)>n+1$.

A-1. Se $k=d(x)>n+1$ é finito, então $\frac{1}{k}<\frac{1}{n+1}$. Como $f(k x) \in \mathbb{T}$, dado que $\mathbb{T}$ é divisível, podemos achar $t_{0} \in \mathbb{T}$ tal que $k t_{0}=f(k x)$. Como $\left|k t_{0}-k s_{n}\right|=\left|k\left(t_{0}-s_{n}\right)\right| \leq 1$, então $\left|t_{0}-s_{n}\right| \leq \frac{1}{k}<\frac{1}{n+1}$. Definimos a aplicação $h^{\prime}: H \cup\{x\} \rightarrow \mathbb{T}$ tomando $h^{\prime} \uparrow_{H}=f \uparrow_{H} \mathrm{e}$ $h^{\prime}(x)=t_{0}$. Note-se que $k h^{\prime}(x)=k t_{0}=f(k x)=h^{\prime}(k x)$, o que permite estender $h^{\prime}$ a um homomorfismo $h: G \rightarrow \mathbb{T}$. Dado que $x \in S$ e $\left|h(x)-s_{n}\right|=\left|t_{0}-s_{n}\right|<\frac{1}{n+1}, h \in U_{n}$; também, $h \uparrow_{H}=f \uparrow_{H}, \operatorname{logo} h \in W\left(f ; y_{1}, \ldots, y_{m}, \varepsilon\right)=W_{1}$, isto é, $U_{n} \cap W_{1} \neq \varnothing$.

A-2. Se $d(x)=\infty$, para cada $i=1 \ldots, m$ definimos $h^{\prime}\left(y_{i}\right)=f\left(y_{i}\right)$ e tomemos $h^{\prime}(x)=$ $s_{n}$. Como para todo $k \in \omega \backslash\{0\} k x \notin H$, podemos estender $h^{\prime}$ para obter um homomorfismo $h: G \rightarrow \mathbb{T}$. Daí, $h \in W_{1}$ e $h \in U_{n}$, pois $\left|h(x)-s_{n}\right|=0<\frac{1}{n+1}$.

B. Para todo $x \in S, d(x) \leq n$. Como $S$ é infinito, podemos supor sem perda de generalidade que para todo $x \in S, d(x)=d \in \omega \backslash\{0\}$. Se $T \in[S]^{\omega}$, vamos mostrar que para cada $k \in \omega$, o conjunto $k T=\{k x: x \in T\}$ é infinito. Por absurdo, suponhamos que existe $T_{0} \in[T]^{\omega}$ de modo que para cada $x \in T_{0}, k x=z_{0}$. Fixando $x_{0} \in T_{0}$, temos que para cada $x \in T_{0}$, $k\left(x-x_{0}\right)=k x-k x_{0}=0$, isto é, $x-x_{0} \in G[k]$, de onde $x \in G[k]+x_{0}$, ou seja, $T_{0} \subseteq G[k]+x_{0}$; como $T_{0} \subseteq T \subseteq S$, então $T_{0} \subseteq S \cap\left(x_{0}+G[k]\right)$, contradizendo isto que $S \cap\left(x_{0}+G[k]\right)$ é infinito.

Suponhamos que para cada $i=1, \ldots, m$, or $\left(y_{i}\right)=d_{i}<\infty$ e tomemos $M=d_{1} \cdots d_{m}$. Como para cada $x \in S$ sabemos que $d x \in H$, temos que $(M d) x=M(d x)=0_{G}$, logo $S \subseteq G[M d]$ o que contradiz que $G$ é quase-livre de torção, pois $S$ é infinito; decorre então que pelo menos um dos elementos $y_{1}, \ldots, y_{m}$ possui ordem infinita. 
De acordo com o anterior, suponhamos que os elementos $y_{1}, \ldots, y_{p}$ tem ordem infinita e que para cada $k=p+1, \ldots, m$, or $\left(y_{k}\right)=d_{k}<\infty$. Se $S=\left\{x_{j}: j \in \omega\right\}$, para cada $j \in \omega$, temos que

$$
d x_{j}=\sum_{k=1}^{m} c_{j, k} y_{k} \quad \text { onde } c_{j, k} \in \mathbb{Z} \text {, e } 0 \leq c_{j, k}<d_{k} \text { se } k=p+1, \ldots, m \text {. }
$$

Notemos que o subgrupo gerado por $\left\{y_{p+1}, \ldots, y_{m}\right\}$ é finito, de modo que se $k \in\{p+1, \ldots, m\}$ os coeficientes $c_{j, k}$ de $d x_{j}$ coincidem num subconjunto infinito $J \in[\omega]^{\omega}$, de modo que se $j \in J$, para todo $p+1 \leq k<m, c_{j, k}=c_{k}$.

Para cada $j \in J$, seja $r(j)=\sum_{i=1}^{p}\left|c_{j, i}\right|$. Observe-se que como $\left\{d x_{j}: j \in J\right\}$ é infinito, o conjunto de $p$-uplas $\left\{\left(c_{j, 1}, \ldots, c_{j, p}\right): j \in J\right\} \subseteq \mathbb{Z}^{p}$ também o é; assim, dado $\delta>0$, existe $j \in J$ tal que $r(j)>\frac{1}{\delta}$. Para tal $j \in J$, podem ser escolhidos $t_{1}, \ldots, t_{p} \in \mathbb{T}$ satisfazendo:

i. para cada $i=1, \ldots, p,\left|t_{i}-f\left(y_{i}\right)\right|<\delta$, e,

ii. $\sum_{i=1}^{p} c_{j, i} t_{i}=d s_{n}-\sum_{i=p+1}^{m} c_{i} f\left(y_{i}\right)$

Definimos então

$$
h^{\prime}\left(y_{i}\right)= \begin{cases}t_{i} & \text { se } i=1, \ldots, p \\ f\left(y_{i}\right) & \text { se } i=p+1, \ldots, m\end{cases}
$$

e, por último, tomemos $h^{\prime}\left(x_{j}\right)=s_{n}$.

Pela propriedade ii), temos

$$
\begin{aligned}
h^{\prime}\left(d x_{j}\right) & =h^{\prime}\left(\sum_{i=1}^{p} c_{j, i} y_{i}+\sum_{i=p+1}^{m} c_{i} y_{i}\right) \\
& =\sum_{i=1}^{p} c_{j, i} h^{\prime}\left(y_{i}\right)+\sum_{i=p+1}^{m} c_{i} h^{\prime}\left(y_{i}\right) \\
& =\sum_{i=1}^{p} c_{j, i} t_{i}+\sum_{i=p+1}^{m} c_{i} f\left(y_{i}\right) \\
& =d s_{n}=d h^{\prime}\left(x_{j}\right)
\end{aligned}
$$

Em conseqüência, $h^{\prime}$ pode ser estendida a um homomorfismo $h: G \rightarrow \mathbb{T}$. Como $x_{j} \in S$ e $h\left(x_{j}\right)=s_{n}$, é claro que $h \in U_{n}$. Agora, para cada $i=1, \ldots, p$ a condição i) implica que $\left|h\left(y_{i}\right)-f\left(y_{i}\right)\right|=\left|t_{i}-f\left(y_{i}\right)\right|<\delta, \operatorname{logo} h \in W\left(f ; y_{1}, \ldots, y_{p}, \delta\right)=W_{1}$. Daí, $W_{1} \cap U_{n} \neq \varnothing$.

Dos casos anteriores, decorre que para cada $n \in \omega, U_{n}$ é denso. 
Notemos que se $G$ é um grupo abeliano quase livre de torção e $S \in[G]^{\omega}$, para cada $x \in G$ e $n \in \omega \backslash\{0\}$ temos $|S \cap(x+G[n])| \leq|G[n]|<\omega$, logo estamos nas condições do lema anterior; daí, usando o teorema de categoria de Baire, obtemos o seguinte resultado:

Lema 5.3.18. Seja $G$ um grupo abeliano infinito quase livre de torção. Se $\left\{S_{\alpha}: \alpha<\kappa\right\} \subseteq[G]^{\omega} e$ $H=\left\{f \in \hat{G}:\right.$ para todo $\alpha<\kappa, f\left(S_{\alpha}\right)$ é denso em $\left.\mathbb{T}\right\}$, então:

i. se $\kappa<\omega_{1}$, H é denso em $\hat{G}$;

ii. Assumindo MA, se $\kappa<\mathfrak{c}$, então $H$ é denso em $\hat{G}$.

Demonstração. Para cada $\alpha<\kappa$, seja $H_{\alpha}=\left\{h \in \hat{G}: h\left(G_{\alpha}\right)\right.$ é denso em TT . Naturalmente, $H=\bigcap_{\alpha<\kappa} H_{\alpha}$.

i. Pelo lema 5.3.17, para cada $\alpha<\kappa$ existe uma família enumerável de abertos densos em $\hat{G}$, digamos $\left\{H_{\alpha, n}: n \in \omega\right\}$ tal que $H_{\alpha}=\bigcap_{n \in \omega} H_{\alpha, n}$. Notemos então, que $\alpha<\omega_{1}$ implica que a coleção de abertos densos em $\mathbb{T},\left\{H_{\alpha, n}: n \in \omega, \alpha<\kappa\right\}$ é também enumerável, de onde

$$
H=\bigcap_{\alpha<\kappa} H_{\alpha}=\bigcap_{\substack{\alpha<\mathcal{K} \\ n \in \omega}} H_{\alpha, n}
$$

é interseção de abertos densos em $\hat{G}$. Note-se que o grupo dual de Pontryagin $\hat{G}$ de $G$ é compacto se consideramos em $G$ a topologia discreta; daí $\hat{G}$ é um espaço Čech-completo e portanto neste espaço vale o Teorema de Categoria de Baire; daí, $H$ é denso em $\hat{G}$.

ii. Suponhamos que $\kappa<\mathfrak{c}$. Como $\hat{G}$ é compacto, em virtude do teorema de IvanovskiiKuz'minov tem-se que $\hat{G}$ é diádico, mais explicitamente, existe uma função contínua e sobrejetora $\phi: 2^{w(\hat{G})} \rightarrow \hat{G}$; pela proposição 1.2 .18 , temos que $\mathrm{c}\left(2^{w(\hat{G})}\right) \leq \omega$, $\operatorname{logoc}(\hat{G}) \leq \omega$.

Pelo lema 5.3.17, para cada $\alpha<\kappa$ temos que $H_{\alpha}$ é interseção enumerável de conjuntos abertos densos de $\hat{G}$, logo $\hat{G} \backslash H_{\alpha}$ é união enumerável de conjuntos raros; como $\hat{G} \backslash H=$ $\bigcup_{\alpha<\kappa} G \backslash H_{\alpha}$ é reunião enumerável de no máximo $\kappa$ conjuntos raros dado que $\kappa<\mathfrak{c}$ e o espaço compacto $\hat{G}$ possui celularidade enumerável, de acordo com a versão topológica de MA, $\hat{G} \backslash H$ é de primeira categoria, ou seja, $H$ é denso em $\hat{G}$.

Lema 5.3.19. Seja $G$ um grupo abeliano quase livre de torção e $N \leq G$. Então, existe $K \leq G$ tal que $N \subseteq K,|K| \leq|N| \omega$ e $G / K$ é livre de torção. 
Demonstração. Observe-se que como $G$ é quase livre de torção, o subgrupo de torção $H$ := $\operatorname{tor}(G)=\bigcup_{n \in \mathbb{N}} G[n]$ é enumerável. Para cada $g \in G$, seja

$$
H(g)=\{x \in G: \text { existe } n \in \mathbb{Z} \backslash\{0\} \text { tal que } n x=g\}
$$

Fixemos $g \in G$ e tomemos $x \in H(g)$ de modo que $n_{x} x=g$. Notemos que para cada $y \in G\left[n_{x}\right]$, $n_{x}(x+y)=n_{x} x+n_{x} y=g+0=g$, isto é, $x+y \in H(g)$; daí, se $x \in H(g), x+G\left[n_{x}\right] \subseteq H(g)$. Percebendo que $n_{x} \in \mathbb{Z} \backslash\{0\}$ é único para cada $x \in H(g)$, pode-se concluir que $|H(g)| \leq|H| \cdot \omega \leq$ $\omega$. Seja $K=\bigcup_{g \in N} H(g)$. Notemos que $N \subseteq K$ e $|K| \leq|N| \cdot \omega$; além disso, se $x, y \in K$, então $m x,-n y \in N$ para alguns $m, n \in \mathbb{Z} \backslash\{0\}$, logo $m n(x-y)=m n x-m n y \in N$, isto é $x-y \in K$, ou seja, $K \leq G$. Resta mostrar que $G / K$ é livre de torção. Seja $x \in G$ e suponhamos que existe $m \in \mathbb{Z} \backslash\{0\}$ tal que $y=m x \in K$. Então, existem $g \in N$ e $n \in \mathbb{Z} \backslash\{0\}$ tais que $n y=g$, mas daí $n(m x)=(n m) x=g$, isto é, $x \in H(g) \subseteq K$.

Teorema 5.3.20. Seja $(G, \tau)$ um grupo topológico abeliano quase livre de torção tal que $|G|=\mathfrak{c}=$ $|\tau|$. Assumindo MA, existe $\sigma \in \mathscr{L}(G)$ de modo tal que:

i. $\tau$ e $\sigma$ são $T_{1}$-independentes;

ii. o grupo $(G, \sigma)$ é de van Douwen.

Demonstração. Começamos por fixar algumas notações. Para cada $v<\mathfrak{c}$, representamos por $\pi_{v}: \mathbb{T}^{c} \rightarrow \mathbb{T}$ a projeção no $v$-ésimo fator. Dado $A \subseteq \mathfrak{c}$, usaremos também a notação $p_{A}: \mathbb{T}^{c} \rightarrow \mathbb{T}^{A}$ para representar a projeção de $\mathbb{T}^{c}$ ao espaço $\mathbb{T}^{A}$. Em particular, se $\alpha<\mathfrak{c}, p_{\alpha}$ é a projeção de $\mathbb{T}^{\mathrm{c}}$ no espaço $\mathbb{T}^{\alpha}$.

A idéia da prova, é construir um homomorfismo $h: G \rightarrow \mathbb{T}^{\mathrm{c}}$ satisfazendo as hipóteses do lema 5.3.15 produzindo desta maneira uma topologia de grupo $T_{1}$-independente de $\tau$. A construção será por indução definindo em cada etapa $\alpha<\mathfrak{c}$ um homomorfismo $h_{\alpha}: G \rightarrow$ $\mathbb{T}$ para, finalmente, obter $h$ como produto diagonal da família $\left\{h_{\alpha}: \alpha<\mathfrak{c}\right\}$. Contudo, o estágio $\alpha$-ésimo da indução implica um trabalho não muito simples. De fato, nesse estágio será definida uma família compatível de homomorfismos parciais $\left\{h_{\beta, v} \in \operatorname{hom}\left(N_{v}, \mathbb{T}\right): \beta<\right.$ $\alpha, \omega \leq v \leq \alpha\}$, isto é, se $\omega \leq \mu<v, h_{\beta, v}$ estende $h_{\beta, \mu}$, o que torna possível tomar $h_{\beta}=\bigcup_{\omega \leq v \leq c} h_{\beta, v}$.

A construção requer um trabalho preliminar, envolvendo, como vimos antes, certas enumerações construídas de maneira conveniente. Primeiro, considere o subgrupo de torção de $G, \operatorname{tor}(G)=\bigcup_{n \in \omega} G[n]$. Pelo lema 5.3.19, tomando $N_{\omega}=\operatorname{tor}(G)$, temos que existe $N_{\omega+1} \leq G$ tal que $N_{\omega} \subseteq N_{\omega+1},\left|N_{\omega+1}\right|=\omega=|\omega+1|$ e $G / N_{\omega+1}$ é livre de torção. Por indução, seja $\alpha<\mathfrak{c}$ e suponhamos que para cada $\omega<v<\alpha$, existem subgrupos $N_{v}$ de $G$ tais que $\left|N_{v}\right|=|v|, G / N_{v}$ 
é livre de torção. Se $\alpha=\beta+1$ para algum ordinal $\beta$, aplicando o lema 5.3.19 ao subgrupo $N_{\beta}$, existe $N_{\beta+1}=N_{\alpha} \leq G$ tal que $N_{\beta} \subseteq N_{\alpha},\left|N_{\alpha}\right| \leq\left|N_{\beta}\right| \cdot \omega=|\beta| \cdot \omega=|\beta|=|\alpha|$ e $G / N_{\alpha}$ é livre de torção. Agora, se $\alpha$ é um ordinal limite, seja $N_{\alpha}=\bigcup_{\omega \leq \mu<\alpha} N_{\mu}$. É claro que $N_{\alpha} \leq G$, e $\left|N_{\alpha}\right| \leq \sum_{\omega \leq \mu<\alpha}|\mu| \leq|\alpha|$; resta mostrar que $G / N_{\alpha}$ é livre de torção; seja $x \in G$ e suponhamos que existe $n \in \omega \backslash\{0\}$ tal que $n x \in N_{\alpha}$; em virtude da construção existe $\mu<\alpha$ tal que $n x \in \mu$, logo, como $G / N_{\mu}$ é livre de torção, $x \in N_{\mu} \subseteq N_{\alpha}$; portanto, $G / N_{\alpha}$ é livre de torção.

Para cada $x \in G$, seja $\xi(x)=\min \left\{\xi \in \mathfrak{c} \backslash \omega: x \in N_{\xi}\right\}$. De acordo com a forma em que a seqüência de subgrupos $\left\{N_{v}: \omega \leq v<\mathfrak{c}\right\}$ foi definida, observe que $\xi(x)$ é um ordinal sucessor ou $\xi(x)=\omega$.

Uma das hipóteses que estamos a assumir, é que $|\tau|=\mathfrak{c}$; essa condição implica que podemos escolher uma enumeração $\left\{F_{\alpha}: \alpha<\mathfrak{c}\right\}$ de todos os subconjuntos fechados de $(G, \tau)$ de cardinalidade c. Para cada $\alpha<\mathfrak{c}$, seja $A_{\alpha}=\left\{\xi(x): x \in F_{\alpha}\right\}$. Se existe $\alpha<\mathfrak{c}$ tal que $\left|A_{\alpha}\right|<\mathfrak{c}$, então existe $F \in\left[F_{\alpha}\right]^{\mathfrak{c}}$ de modo que para todo $x \in F, \xi(x)=\beta+1<\mathfrak{c}$. Daí, $F \subseteq N_{\beta+1}$, $\operatorname{logo}$ $\mathfrak{c}=|F| \leq\left|N_{\beta+1}\right|=|\beta|<\mathfrak{c}$, uma contradição. Conclui-se que para todo $\alpha<\mathfrak{c},\left|F_{\alpha}\right|=\mathfrak{c}$.

Consideremos um refinamento $\left\{B_{\alpha}: \alpha<\mathfrak{c}\right\}$ da família $\left\{A_{\alpha}: \alpha<\mathfrak{c}\right\}$ de modo que as seguintes condições sejam satisfeitas para cada $\beta<\alpha<\mathfrak{c}$ :

$\diamond B_{\alpha} \in\left[A_{\alpha}\right]^{c}$

$\diamond B_{\alpha} \cap B_{\beta}=\varnothing$

$\diamond L=\bigcup_{\alpha<c} B_{\alpha}=\bigcup_{\alpha<c} A_{\alpha}$

Notemos que $\omega \in L$, pois $\xi(0)=\omega$. Seja $\omega<\alpha<\mathfrak{c}$ e suponhamos que $\alpha+1 \notin L$, de modo que $N_{\alpha}=N_{\alpha+1}$. Pela construção de $N_{\alpha+1}$, isto sugere que se $N \leq G$ é tal que $N_{\alpha} \subseteq N, G / N$ é livre de torção e $|N| \leq\left|N_{\alpha}\right| \cdot \omega=|\alpha| \cdot \omega$, então $N=N_{\alpha}$. Daí, para todo $\beta>\alpha, N_{\beta}=N_{\alpha}, \log \sigma=N_{\alpha}$ de onde $|G|=|\alpha|<\mathfrak{c}$, uma contradição. Decorre então que $L \backslash\{\omega\}=\{\alpha+1: \omega \leq \alpha<\mathfrak{c}\}$.

A seguir, vamos construir uma função sobrejetora $\varphi: L \rightarrow \mathfrak{c} \times \mathfrak{c}$ satisfazendo:

A- 1) se $\varphi(\alpha)=(\beta, \delta)$ então $\alpha \in B_{\beta}$ e $\delta \leq \alpha$

A- 2) $\varphi(\omega)=(\beta, 0)$ para algum $\beta<\mathfrak{c}$

Em primeiro lugar, para cada $\beta<\mathfrak{c}$ podemos fixar um $\leq$-isomorfismo $\psi_{\beta}: \mathfrak{c} \rightarrow B_{\beta}$, de modo que por se tratar de uma função não decrescente, para cada $\gamma<\mathfrak{c}$ satisfaz $\gamma \leq \psi_{\beta}(\gamma)$. Fazendo uso desse isomorfismo de ordem, para cada $\alpha \in B_{\beta}$ pode-se definir $\varphi(\alpha)=\left(\beta, \psi_{\beta}^{-1}(\alpha)\right)$; observe-se que $\varphi$ já satisfaz a condição A-1). De outra parte, para cada $(\beta, \gamma) \in \mathfrak{c} \times \mathfrak{c}$, podemos tomar $\xi \in B_{\beta}$ tal que $\psi_{\beta}^{-1}(\xi)=\gamma$ e assim, $\varphi(\xi)=(\beta, \gamma)$; portanto, $\varphi(L)=\mathfrak{c} \times \mathfrak{c}$. 
Como $\omega \in L$, existe $\beta<\mathfrak{c}$ tal que $\omega \in B_{\beta}$, $\operatorname{logo} \omega=\min B_{\beta}$ e assim $\omega=\psi_{\beta}(0)$; de acordo com a definição, tem-se que $\varphi(\omega)=(\beta, 0)$. Portanto, a função $\varphi$ satisfaz também a condição A-2).

Seja $\mathcal{B}$ a base enumerável de $\mathbb{T}$ que consta dos arcos abertos com extremos racionais. Seja $\mathcal{U}$ a família de todos os abertos canônicos $O \in \mathbb{T}^{\mathbb{C}}$ tais que $\pi_{\alpha}(O) \in \mathcal{B}$ para cada $\alpha<\mathfrak{c}$. Para cada $O \in \mathcal{U}$, associamos o conjunto não vazio $\operatorname{coord}(O)=\left\{\alpha<\mathfrak{c}: \pi_{\alpha}(O) \neq \mathbb{T}\right\} \in[\mathfrak{c}]^{<\omega}$. Podemos tomar uma enumeração $\left\{O_{\delta}: \delta<\mathfrak{c}\right\}$ da família $\mathcal{U}$ de maneira que $\operatorname{coord}\left(O_{\delta}\right) \subseteq \delta$ para cada $\delta<\mathfrak{c}$, onde $O_{0}=\mathbb{T}^{c}$. Note-se que para cada $\delta<\mathfrak{c}$ existem exatamente $\omega$ abertos $O \in \mathcal{U}$ satisfazendo $\operatorname{coord}(O) \subseteq \delta$.

Para poder garantir a existência de pontos de acumulação, convém também escolher uma enumeração de $[G]^{\omega}$, digamos $\left\{S_{\mu}: \omega \leq \mu<\mathfrak{c}\right\}$ satisfazendo para cada $\omega \leq \mu<\mathfrak{c}$, supp $S_{\mu}=$ $\left\{\xi(x): x \in S_{\mu}\right\} \subseteq \mu$.

Seja $\mathbb{S}=\left\{t \in \mathbb{T}^{\mathfrak{c}}:|\operatorname{supp} t|<\mathfrak{c}\right\}$ e, para cada $\omega \leq \alpha<\mathfrak{c}$ definimos $C_{\alpha}=\left\{t \in \mathbb{T}^{\mathfrak{c}}: \operatorname{supp} t \subseteq \alpha\right\}$. Pelo teorema de Martin-Solovay, temos que MA implica que $\mathfrak{c}$ é um cardinal fortemente limite, logo para cada $\omega \leq \alpha<\mathfrak{c}$, tem-se $\left|C_{\alpha}\right|=\left|\mathbb{T}^{\alpha}\right|=\left|\mathfrak{c}^{\alpha}\right|=\left|\left(2^{\alpha}\right)^{\alpha}\right|=\mathfrak{c}$. Como $\mathbb{S}=\bigcup_{\omega \leq \alpha<\mathfrak{c}} C_{\alpha}$, temos que $|\mathbb{S}| \leq \mathfrak{c}$. Notemos que para cada $\alpha<\mathfrak{c}$, a função caraterística $\chi_{\alpha}: \mathfrak{c} \rightarrow \mathbb{T}$ está em $\mathbb{S}$, logo, a cardinalidade de $\mathbb{S}$ é exatamente c. Com isto, podemos tomar uma enumeração $\mathbb{S}=\left\{b_{\alpha}: \alpha<\mathfrak{c}\right\}$ de maneira tal que para cada $f \in \mathbb{S},\left|\left\{\alpha<\mathfrak{c}: b_{\alpha}=f\right\}\right|=\mathfrak{c}$; usaremos esta enumeração para mostrar que para cada $\alpha<\mathfrak{c}, p_{\alpha}(h(G))=\mathbb{T}^{\alpha}$.

\section{Construção do homomorfismo $h$}

A continuação, vamos definir indutivamente uma família $\left\{h_{\alpha, v}: \alpha<\mathfrak{c}, \omega \leq v<\mathfrak{c}\right\}$ satisfazendo, para cada $\alpha<\mathfrak{c}, \omega \leq v<\mathfrak{c}$ :

B-1. $h_{\alpha, v} \in \operatorname{hom}\left(N_{v}, \mathbb{T}\right)$, e, para cada $\omega \leq \mu<v, h_{\alpha, v}$ estende $h_{\alpha, \mu}$;

B-2. a imagem $\Delta_{\mu \leq \gamma<\alpha} h_{\gamma, v}\left(S_{\mu}\right)$ é densa em $\mathbb{T}^{\alpha \backslash \mu}$ sempre que $\mu<\alpha$ e $\omega \leq \mu<v$;

B-3. para cada $\gamma<v$, existe um ponto $x \in N_{v+1} \backslash N_{v}$ tal que $h_{\gamma, v+1}(x)=b_{v}(x)$, e,

B-4. se $\alpha \in L, \varphi(\alpha)=(\beta, \delta)$ e $\operatorname{coord}\left(O_{\delta}\right)=\left\{\mu_{1}, \ldots, \mu_{k}\right\}$, então existe um ponto $y \in F_{\beta}$ tal que $\xi(y)=\alpha$ e $h_{\mu_{i}, \alpha}(y) \in \pi_{\mu_{i}}\left(O_{\delta}\right)$ para cada $i=1, \ldots, k$.

Como o subgrupo tor $(G)$ = é enumerável, podemos achar uma família de homomorfismos $\mathcal{H}_{\omega}=\left\{h_{k, \omega}: k \in \omega\right\} \subseteq \operatorname{hom}\left(N_{\omega}, \mathbb{T}\right)$ que separa os elementos de $N_{\omega}$, isto é, para cada $x \in G \backslash\left\{0_{G}\right\}$, existe $k^{\prime}<\omega$ tal que $h_{k^{\prime}, \omega}(x) \neq 0$. Com efeito, suponhamos que $N_{\omega} \backslash\left\{0_{G}\right\}=\left\{x_{i}: i \in \omega\right\}$, 
e, fixemos $i \in \omega$. Se $\operatorname{or}(G) x_{i}=n$, podemos escolher $y_{i} \in \mathbb{T}$ de modo que or $(G) y_{i}=n$ e definimos $\tilde{h}\left(x_{i}\right)=y_{i}$. Note-se que temos definido $\tilde{h} \in \operatorname{hom}\left(\left\langle x_{i}\right\rangle, \mathbb{T}\right)$. Como $\mathbb{T}$ é divisível, $\tilde{h}$ pode ser estendido a um homomorfismo $h_{i, \omega}: N_{\omega} \rightarrow \mathbb{T}$, e, obviamente $h_{i, \omega}\left(x_{i}\right) \neq 0$, como era pretendido.

Naturalmente, $\mathcal{H}_{\omega}$ satisfaz as condições B-1)-B-3) trivialmente. Agora, como $\varphi(\omega)=(\beta, 0)$ para certo $\beta<\mathfrak{c}$ e $O_{0}=\mathbb{T}$, temos que coord $\left(O_{0}\right)=\varnothing$, de modo que a condição B-4) é também satisfeita pela família $\mathcal{H}_{\omega}$, verificando-se deste modo o primeiro passo da nossa indução.

Seja agora $\omega<\alpha<\mathfrak{c}$ e suponhamos que para todo $\beta<\alpha$, a família $\mathcal{H}_{\beta}=\left\{h_{\gamma, v}: \gamma<\beta, \omega \leq\right.$ $v \leq \beta\}$ tem sido definida de maneira que $\mathcal{H}_{\beta}$ satisfaz as condições B-1)-B-4).

Se $\alpha$ é um ordinal limite, sabemos que $N_{\alpha}=\bigcup_{\omega \leq \beta<\alpha} N_{\beta}$, e pela condição B-1) temos que para todo $\gamma<\alpha$, existe $h_{\gamma, \alpha} \in \operatorname{hom}\left(N_{\alpha}, \mathbb{T}\right)$ tal que $h_{\gamma, \alpha} \uparrow_{N_{\beta}}=h_{\gamma, \beta}$ sempre que $\omega \leq \beta<\alpha$, em conseqüência, a família $\mathcal{H}_{\alpha}=\left\{h_{\gamma, v}: \gamma<\alpha, \omega \leq v \leq \alpha\right\}$ satisfaz a condição B-1). Como $\mathcal{H}_{\beta}$ satisfaz as condições B-1)-B-4), é imediato que $\mathcal{H}_{\alpha}$ satisfaz também as condições B-2)-B-4).

Por fim, suponhamos que $\alpha=\beta+1$ para algum $\beta<\mathfrak{c}$. Para definir a família $\mathcal{H}_{\alpha}$, é preciso estender o homomorfismo $h_{\gamma, \beta} \in \mathcal{H}_{\beta}$ sobre $N_{\alpha}$ para cada $\gamma<\beta$, obtendo dessa maneira os homomorfismos $h_{\gamma, \alpha}$. Além disso, é preciso construir o homomorfismo $h_{\beta, \alpha}$ definido sobre $N_{\alpha}$.

Começamos por construir os homomorfismos $h_{\gamma, \beta}$. Se $\varphi(\alpha)=(\gamma, \delta)$, tem-se que $\alpha \in B_{\gamma} \subseteq$ $A_{\gamma}=\left\{\xi(x): x \in F_{\gamma}\right\}$, logo existe $y \in F_{\gamma}$ tal que $\xi(y)=\alpha$ de maneira que $y \in N_{\alpha} \backslash N_{\beta}$. Se $\operatorname{coord}\left(O_{\delta}\right)=\left\{\mu_{1}, \ldots, \mu_{k}\right\}$, lembrando que $\operatorname{coord}\left(O_{\delta}\right) \subseteq \delta \leq \alpha$ temos para cada $i=1, \ldots, k$, $\mu_{i}<\alpha$. Seja $O_{\delta, i}=\pi_{\mu_{i}}\left(O_{\delta}\right) \subseteq \mathbb{T}$. De acordo com a condição B-4), para cada $\gamma<\beta$ temos que definir uma extensão $h_{\gamma, \alpha}$ de $h_{\gamma, \beta}$ satisfazendo

$$
h_{\mu_{i}, \alpha}(y) \in O_{\delta, i}=\pi_{\mu_{i}}\left(O_{\delta}\right) \quad i=1, \ldots, k
$$

Também, temos que escolher um elemento $x \in N_{\alpha} \backslash N_{\beta}$ de modo que

$$
h_{\gamma, \alpha}(x)=b_{\beta}(\gamma) \quad \text { para cada } \gamma<\beta \quad(* *)
$$

Como $G / N_{\beta}$ é livre de torção, podemos escolher qualquer $x \in N_{\alpha} \backslash N_{\beta}$ e definir uma extensão $h_{\gamma, \alpha}$ de $h_{\gamma, \beta}$ satisfazendo (**) para cada $\gamma \in \beta \backslash\left\{\mu_{1}, \ldots, \mu_{k}\right\}$. Resta mostrar que isto também é possível para cada $\gamma \in\left\{\mu_{1}, \ldots, \mu_{k}\right\}$, ou, de maneira equivalente, mostrar que as condições $(*)$ e $(* *)$ são compatíveis. Dado que $\mathbb{T} \cong \mathbb{R} / \mathbb{Z}$, é possível definir a aplicação $\ell: \mathcal{B} \rightarrow[0,1]$, que associa a cada arco aberto em $\mathbb{T}$ o seu comprimento, de modo que $\ell(\mathbb{T})=1$. 
Seja $a \in \mathbb{T}, n \in \omega \backslash\{0\}$ e $U \in \mathcal{B}, U \neq \varnothing$. Consideremos o sistema

$$
\left\{\begin{array}{l}
n z=a \\
z \in U
\end{array}\right.
$$

Como $\mathbb{T}$ é um grupo divisível, existe $b \in \mathbb{T}$ tal que $n b=a$. Se supomos que $\frac{1}{n}<\ell(U), n U=\mathbb{T}$, $\operatorname{logo} b \in U$; portanto, o sistema considerado possui solução em $U$.

Seja agora $n \in \omega \backslash\{0\}$ de modo que para cada $i=1, \ldots, k, \frac{1}{n}<\ell\left(O_{\delta, i}\right)$. Se $y \in N_{\alpha} \backslash N_{\beta}$, $y \in F_{\alpha}$ é tal que $x=n y$, de acordo $\operatorname{com}(*)$ e $(* *)$ se satisfaz, para cada $i=1, \ldots, k$,

$$
\left\{\begin{array}{l}
h_{\mu_{i}, \alpha}(n y)=n h_{\mu_{i}, \alpha}(y)=b_{\beta}\left(\mu_{i}\right) \\
h_{\mu_{i}, \alpha}(y) \in O_{\delta, i}=\pi_{\mu_{i}}\left(O_{\delta}\right)
\end{array}\right.
$$

Como $\frac{1}{n}<\ell\left(O_{\delta, i}\right)$ para cada $i=1, \ldots, k$, é possível escolher uma solução $z_{i}=h_{\mu i, a}(y) \in O_{\delta, i}$ deste sistema. Então, definimos para cada $i=1, \ldots, k, h_{\mu_{i}, \alpha} \in \operatorname{hom}\left(N_{\alpha}, \mathbb{T}\right)$ satisfazendo $h_{\mu_{i}, \alpha} \uparrow_{N_{\beta}}=h_{\mu_{i}, \beta}$ e $h_{\mu_{i}, \alpha}(y)=z_{i}$, com o qual garantimos que B-3) e B-4) são satisfeitas por $\alpha=\beta+1$. Vamos construir o homomorfismo $h_{\beta, \alpha}$. Para cada $\mu<\beta$, seja $f_{\mu, \beta}=\Delta\left\{h_{\gamma, \beta}: \mu \leq \gamma<\beta\right\}$; como foi observado em outras construções, claramente $f_{\mu, \beta} \in \operatorname{hom}\left(N_{\beta}, \mathbb{T}^{\beta \backslash \mu}\right)$.

Seja $\mathcal{U}_{\mu, \beta}$ a família de todos os abertos canônicos no espaço $\mathbb{T}^{\beta \backslash \mu}$ tal que para cada $U \in \mathcal{U}_{\mu, \beta}$, $\pi_{\alpha}(U) \in \mathcal{B}$ para todo $\alpha \in \beta \backslash \mu$. Como $\mathcal{B}$ é enumerável, note-se que $\left|\mathcal{U}_{\mu, \beta}\right| \leq|\beta|$ para cada $\mu<\beta$, de modo que a cardinalidade da família

$$
\mathcal{K}_{\beta}=\left\{S_{\mu} \cap f_{\mu, \beta}^{-1}\left[U \cap f_{\mu, \beta}\left(S_{\mu}\right)\right]: U \in \mathcal{U}_{\mu, \beta}, \omega \leq \mu<\beta\right\} \cup\left\{S_{\beta}\right\}
$$

não supera $|\beta|$.

Observamos que cada elemento de $\mathcal{K}_{\beta}$ é infinito; de fato, pela condição B-2), $f_{\mu, \beta}\left(S_{\mu}\right)$ é denso em $\mathbb{T}^{\beta \backslash \mu}$, logo $U \cap f_{\mu, \beta}\left(S_{\mu}\right) \subseteq f_{\mu, \beta}\left(S_{\mu}\right)$ é infinito, e assim, $S_{\mu} \subseteq f^{-1}\left[U \cap f_{\mu, \beta}\left(S_{\mu}\right)\right]$ também é.

Seja $\mathcal{J}=\left\{f \in \hat{N}_{\alpha}: f(K)\right.$ é denso em $\mathbb{T}$ para cada $\left.K \in \mathcal{K}_{\beta}\right\}$. Como $\left|\mathcal{K}_{\beta}\right| \leq|\beta|<\mathfrak{c}$, pelo item ii) do lema 5.3.18, podemos achar $h_{\beta, \alpha} \in \operatorname{hom}\left(N_{\alpha}, \mathbb{T}\right)$ de modo que $h_{\beta, \alpha}(K)$ é denso para cada $K \in \mathcal{K}_{\beta}$. Esta definição implica que a condição B-2) é válida no caso $\alpha=\beta+1$. De fato, a densidade de $h_{\beta, \alpha}\left(S_{\beta}\right)$ em $\mathbb{T}^{\alpha \backslash \beta}=\mathbb{T}$ provem da definição do próprio $h_{\beta, \alpha}$.

Agora, para cada $\mu<\beta$, seja $f_{\mu, \alpha}=\Delta_{\mu \leq \gamma<\alpha} h_{\gamma, \alpha}$. Temos que provar que $f_{\mu, \alpha}\left(S_{\mu}\right)$ é denso em $\mathbb{T}^{\alpha \backslash \mu}$; se $U \in U_{\mu, \alpha}$ é um aberto canônico não vazio em $\mathbb{T}^{\alpha \wedge \mu}$, podemos exprimir $U=U_{1} \times V$ onde $U_{1} \in U_{\mu, \beta}$ e $V \subseteq \mathbb{T}$ é aberto. Pela hipótese B-2), sabemos que $f_{\mu, \beta}\left(S_{\mu}\right)$ é denso em $\mathbb{T}^{\beta \backslash \mu}$, logo, se $S=S_{\mu} \cap f_{\mu, \beta}^{-1}\left[U_{1} \cap f_{\mu, \beta}\left(S_{\mu}\right)\right] \in \mathcal{K}_{\beta}$ temos que $h_{\beta, \alpha}(S)$ é denso em $\mathbb{T}$; assim, existe $x \in S$ tal que $h_{\beta, \alpha}(x) \in V$. Dado que $f_{\mu, \beta}(x) \in f_{\mu, \beta}(S) \subseteq U_{1}, f_{\mu, \alpha}(x)=\left(f_{\mu, \beta}(x), h_{\beta, \alpha}(x)\right) \in U_{1} \times V$, ou seja, 
$f_{\mu, \alpha}\left(S_{\mu}\right) \cap U \neq \varnothing$.

Pela construção, é claro que a família $\mathcal{H}_{\alpha}=\left\{h_{\gamma, v}: \gamma<\alpha, \omega \leq v \leq \alpha\right\}$ satisfaz as condições B-1)-B-4), completando-se, desta maneira, o processo indutivo.

A condição B-1), implica que para cada $\alpha<\mathfrak{c}$, o sistema de homomorfismos $\left\{h_{\alpha, \mu}: \omega \leq v<\right.$ c) é compatível, logo, como $G=\bigcup_{\omega \leq v<\mathfrak{c}} N_{v}$ existe $h_{\alpha} \in \operatorname{hom}(G, \mathbb{T})$ tal que para cada $\omega \leq v<\mathfrak{c}$, $h_{\alpha} \uparrow_{N_{v}}=h_{\alpha, v}$. Seja $h=\Delta\left\{h_{\alpha}: \alpha<\mathfrak{c}\right\} \in \operatorname{hom}\left(G, \mathbb{T}^{c}\right)$. A seguir, mostraremos que $h$ satisfaz as hipóteses do lema 5.3.15.

Primeiro, observemos que $h$ é um monomorfismo. Com efeito, seja $x \in G \neq\left\{0_{G}\right\}$ arbitrário. Se $n x=0_{G}$ para algum $n \in \omega \backslash\{0\}, x \in N_{\omega}$; como a família $\left\{h_{k, \omega}: k \in \omega\right\}$ separa pontos, existe $k<\omega$ tal que $h_{k, \omega}(x) \neq 0_{\mathbb{T}}$; daí, $h_{\omega}(x) \neq 0_{\mathbb{T}}$ e portanto $h(x) \neq 0 \in \mathbb{T}^{c}$. Se $x \in G \backslash N_{\omega}$, temos que $\{n x: n \in \mathbb{Z}\} \in[G]^{\omega} \operatorname{logo}$ podemos tomar $\mu<\mathfrak{c}$ de modo que $S_{\mu}=\{n x: n \in \omega\}$. Pela condição B-2), tomando $\alpha=\mu+1$, temos que $h_{\mu, \mu+1}\left(S_{\mu}\right)$ é denso em $\mathbb{T}$, logo para qualquer aberto $U \subseteq \mathbb{T}$ que não contém o elemento $0_{\mathbb{T}}$, existe $m \in \mathbb{Z}$ tal que $h_{\mu, \mu+1}(m x)=m h_{\mu, \mu+1}(x) \in U$, de onde $h_{\mu, \mu+1}(x) \neq 0_{\mathbb{T}}$. Como $S_{\mu} \subseteq N_{\mu}$, tem-se então que $h_{\mu}(x)=h_{\mu, \mu+1} \uparrow_{\nu_{\mu}}(x) \neq 0_{\mathbb{T}}$, logo $h(x) \neq 0 \in \mathbb{T}^{c}$.

Agora, para garantir que $h(G)$ é um grupo de van Douwen é suficiente ver que $h$ satisfaz as seguintes duas propriedades:

C-1. $h(G) \leq \mathbb{T}^{c}$ é hereditariamente finalmente denso em $\mathbb{T}^{c}$, isto é, dado $S \in[G]^{\omega}$, existe $\alpha<\mathfrak{c}$ tal que $p_{c \wedge \alpha}(h(S))$ é denso em $\mathbb{T}^{c \backslash \alpha}$;

C-2. para cada $\alpha<\mathfrak{c}, p_{\alpha}(h(G))=\mathbb{T}^{\alpha}$.

A propriedade C-1) implica que $h(G)$ não possui seqüências não triviais convergentes. Com efeito, suponhamos por absurdo que existe uma seqüência injetora $\left\{y_{n}: n \in \omega\right\} \subseteq h(G)$ de modo que $\lim _{n \in \omega} y_{n}=y \in h(G)$. Observemos que considerado como subespaço de $\mathbb{T}^{c}$, o conjunto $Y=\left\{y_{n}: n \in \omega\right\} \cup\{y\}$ é fechado, e assim, compacto. Como $S=\left\{h^{-1}\left(y_{n}\right): n \in \omega\right\} \subseteq G$ é infinito, a propriedade C-1) implica que existe $\alpha<\mathfrak{c}$ tal que $p_{\llcorner\wedge \alpha}(h(S))=p_{\llcorner\wedge \alpha}\left(\left\{y_{n}: n \in \omega\right\}\right)$ é denso em $\mathbb{T}^{\wedge \wedge \alpha}$. Note-se que pela continuidade das projeções $p_{c \wedge \alpha}(Y)$ é compacto e assim fechado em $\mathbb{T}^{c \backslash \alpha}$ contendo o denso $p_{c \wedge \alpha}\left(\left\{y_{n}: n \in \omega\right\}\right)$, de onde $p_{c \wedge \alpha}(Y)=\mathbb{T}^{c \backslash \alpha}$ uma contradição, pois $\left|p_{c \wedge \alpha}(Y)\right| \leq|Y|=\omega<\mathfrak{c}<2^{\mathfrak{c}} \leq\left|\mathbb{T}^{c \backslash \alpha}\right|$.

De outro lado, as propriedades C-1) e C-2) implicam que $h(G)$ é enumeravelmente compacto, como mostra o trabalho [39]. Adicionalmente, notemos que $h(G)$ é denso em $\mathbb{T}^{c}$. Com efeito, dado $F=\left\{\alpha_{1}, \ldots, \alpha_{k}\right\} \subseteq \mathfrak{c}$ onde $\alpha_{1}<\cdots<\alpha_{k}$, consideremos o aberto canônico $\prod_{\alpha \in \mathrm{c}} U_{\alpha} \subseteq \mathbb{T}^{\mathrm{c}}$ tal que para todo $\alpha \notin F, U_{\alpha}=\mathbb{T}$ e $U_{\alpha}$ é um aberto próprio não vazio em $\mathbb{T}$ se $\alpha \in F$. Seja $\tilde{f} \in p_{a_{k+1}}(U)$, isto é, existe $g \in U$ tal que $\tilde{f}=g \uparrow_{a_{k+1}}$. Note-se que pela proprie- 
dade C-2), $p_{a_{k+1}}(h(G))=\mathbb{T}^{\alpha_{k+1}}$, daí, existe também $h \in h(G)$ satisfazendo $\tilde{f}=\pi_{a_{k+1}}(h)$, ou seja, $\tilde{f}=h \uparrow_{a_{k+1}}$. Notemos que, para todo $\beta>\alpha_{k}$ o segmento final $U_{c \wedge \beta}$ é, de fato, $\mathbb{T}^{c \backslash \beta}$, de modo que $h \uparrow_{\imath a_{k+1}} \in U_{\imath\urcorner \beta}$. Daí, $h=h \uparrow_{\alpha_{k+1}} \cup h \uparrow_{c a_{k+1}}=g \uparrow_{a_{k+1}} \cup h \uparrow_{c a_{k+1}} \in U \cap h(G)$.

Dado que a topologia da qual queremos munir ao grupo $G$ é a gerada pelo monomorfismo $h$, o item ii) do teorema decorre das propriedades C-1) e C-2). Vejamos agora que o homomorfismo $h$ verifica, de fato, essas propriedades:

- $h(G)$ satisfaz C-1): Se $S \in[G]^{\omega}$, existe $\mu<\mathfrak{c}$ tal que $S=S_{\mu}$; para cada $\alpha \in(\mu, \mathfrak{c})$, consideremos $f_{\mu, \alpha}=\Delta\left\{h_{\gamma, \alpha}: \mu \leq \gamma \leq \alpha\right\}$. Como $p_{\alpha \backslash \mu} \circ h=f_{\mu, \alpha}$, pela condição B-2) temos que $f_{\mu, \alpha}\left(S_{\mu}\right)=p_{\alpha \curlywedge \mu}\left(h\left(S_{\mu}\right)\right)$ é denso em $\mathbb{T}^{\alpha \backslash \mu}$; dado que isto ocorre para cada $\alpha \in(\mu, \mathfrak{c})$, $p_{c \wedge \mu}\left(h\left(S_{\mu}\right)\right)$ é denso em $\mathbb{T}^{c \wedge \mu}$; portanto, $h(G)$ é HFD em $\mathbb{T}^{c}$.

- $h(G)$ satisfaz C-2): Se $\alpha<\mathfrak{c}$ e $z \in \mathbb{T}^{\alpha}$, observamos que $z$ pode ser considerada como uma função na coleção $\mathbb{S}$, de modo que existe $\beta<\mathfrak{c}$ tal que para cada $\gamma<\alpha, z(\gamma)=b_{\beta}(\gamma)$. Como $b_{\beta}$ aparece $\mathfrak{c}$ vezes nessa enumeração, podemos achar $v \geq \alpha$ tal que $b_{\beta}=b_{v}$, de onde $b_{v}(\gamma)=z(\gamma)$ para cada $\gamma<\alpha$. De acordo com a condição B-3), existe $x \in N_{v+1} \backslash N_{v}$ tal que $h_{\gamma, v+1}(x)=b_{v}(\gamma)=z(\gamma)$ para cada $\gamma<v$. Concluímos então que para cada $\gamma<\alpha$, $h(x)(\gamma)=z(\gamma)$, ou seja $p_{a}(h(x))=z$. Sendo $z \in \mathbb{T}^{\alpha}$ um ponto arbitrário, temos que $p_{\alpha}(h(G))=\mathbb{T}^{\alpha}$.

Agora, mostraremos que para todo subconjunto fechado $F \in[G]^{c}, h(F)$ é denso em $\mathbb{T}^{c}$. Visto que $\left\{F_{\beta}: \beta<\mathfrak{c}\right\}$ é uma enumeração dos conjuntos fechados em $(G, \tau)$ de cardinalidade c e $\left\{O_{\delta}: \delta<\mathfrak{c}\right\}$ enumera os abertos canônicos de $\mathbb{T}^{\mathfrak{c}}$ associados à base $\mathcal{B}$ de $\mathbb{T}$, é suficiente mostrar que para cada $\beta<\mathfrak{c}$ e $\delta<\mathfrak{c}, h\left(F_{\beta}\right) \cap O_{\delta} \neq \varnothing$. Se consideramos a $\varphi: L \rightarrow \mathfrak{c} \times \mathfrak{c}$ como antes, dado que esta função é sobrejetora, podemos tomar $\alpha \in L$ de modo tal que $\varphi(\alpha)=(\beta, \delta)$. De acordo com a construção, temos que $\delta \leq \alpha$; portanto, se coord $\left(O_{\delta}\right)=\left\{\mu_{1}, \ldots, \mu_{k}\right\}$ temos que $\mu_{i}<\alpha$ para cada $i=1, \ldots, k$; logo, segundo a condição B-4), existe $y \in F_{\beta}$ tal que $\xi(y)=\alpha$ e $h_{\mu_{i}, \alpha}(y) \in \pi_{\mu_{i}}\left(O_{\delta}\right)$ para cada $i=1, \ldots, k$. Como $y \in N_{\alpha}$ e $h_{\mu_{i}} \uparrow_{N_{\alpha}}=h_{\mu_{i}, \alpha}$ para todo $i=1, \ldots, k$, decorre que $h(y)\left(\mu_{i}\right) \in \pi_{\mu_{i}}\left(O_{\delta}\right)$; de outra parte $\pi_{\gamma}\left(O_{\delta}\right)=\mathbb{T}$ para cada $\gamma \in \mathfrak{c} \backslash \operatorname{coord}\left(O_{\delta}\right)$. Do anterior, $h(y) \in O_{\delta}$, ou seja, $h(y) \in h\left(F_{\beta}\right) \cap O_{\delta}$.

Finalmente, dado que $h$ verifica as hipóteses do lema 5.3.15 e estamos assumindo MA, a topologia $\sigma=\left\{h^{-1}(U \cap h(G)): U\right.$ é aberto em $\left.\mathbb{T}^{c}\right\}$ definida sobre $G$ é independente de $\tau$.

Corolário 5.3.21. (MA) Se $(G, \tau)$ é um grupo topológico abeliano quase livre de torção tal que $w(G)<\mathfrak{c}=|G|$ então existe $\sigma \in \mathscr{L}(G)$ tal que $\tau$ e $\sigma$ são $T_{1}$-independentes. 
Demonstração. Pela proposição 1.2.16, temos que $|\tau| \leq 2^{w(G)}$. Como $w(G)<\mathfrak{c}, 2^{w(G)}=\mathfrak{c}$ em virtude de MA, de onde $|\tau| \leq \mathfrak{c}$ e podemos aplicar o teorema 5.3.20.

Corolário 5.3.22. (MA) Cada um dos grupos $(\mathbb{R},+),(\mathbb{T},+),(\mathbb{C},+)$ e $\left(\mathbb{C}^{*}, \times\right)$ considerado com a topologia euclidiana usual respectiva, admite uma topologia de grupo $T_{1}$-independente munido da qual é um grupo de van Douwen.

Demonstração. Note-se que se $G$ é qualquer dos grupos mencionados acima e $\tau$ é a topologia euclidiana usual definida nele, temos $|G|=\mathfrak{c}=|\tau|$. Como conseqüência do teorema 5.3.20, obtemos uma topologia $\sigma \in \mathscr{L}(G)$ que é $T_{1}$-independente de $\tau$. Como a topologia $\tau$ provem de uma métrica, $\tau$ é seqüencial, logo pela proposição 5.3 .12 temos que $(G, \sigma)$ é enumeravelmente compacto e sem seqüências não triviais convergentes.

Nota 5.3.23. Vale a pena salientar que na prova acima, a única propriedade topológica que usamos do grupo $(G, \tau)$ foi que a família de todos os subconjuntos fechados nesse espaço tem cardinalidade $\mathfrak{c}$. Daí, o teorema anterior permanece válido se o grupo $(G, \tau)$ onde $|G|=\mathfrak{c}$, ao invés de satisfazer $|\tau|=\mathfrak{c}$ é, simplesmente, hereditariamente separável.

Particularmente, estamos nessa situação se o espaço $(G, \tau)$ satisfaz $n w(G) \leq \omega$; de fato, suponhamos que $S \subseteq G$ e $\mathscr{A}=\left\{A_{n}: n \in \omega\right\}$ é uma rede para $S$, isto é, qualquer aberto em $S$ é união de elementos de $\mathscr{A}$. Para cada $n \in \omega$, fixemos $a_{n} \in A_{n}$; dado $U \subseteq S$ aberto, existe $k \in \omega$ de modo que $A_{k} \subseteq U$, $\log a_{k} \in U$; portanto, o conjunto $D=\left\{a_{n}: n \in \omega\right\}$ é denso em $S$, ou seja, $G$ é hereditariamente separável.

Exemplo 5.3.24. O teorema 5.3.20 não pode ser estendido aos grupos abelianos infinitos de ordem finita. Com efeito, se $p, q \in \mathbb{Z}$ são números primos distintos, sejam $H(p)$ e $H(q)$ dois grupos abelianos infinitos de ordens $p$ e $q$ respectivamente. Seja $G=H(p) \oplus H(q)$, e, suponhamos que $\sigma, \tau \in \mathscr{L}(G)$. Seja $f: G \rightarrow G$ o homomorfismo dado por $f(x)=p x$; dado que $f$ é contínuo em qualquer topologia de grupo $T_{1}$ considerada sobre $G, f^{-1}\left(\left\{0_{G}\right\}\right)=$ $\operatorname{ker}(f)=H(p) \times\{0\}$ é fechado nos espaços $(G, \tau)$ e $(G, \sigma)$. Como $H(p)$ é infinito, e as topologias que estamos considerando são de Hausdorff, decorre que $\sigma$ e $\tau$ não podem ser independentes; dado que $\tau$ e $\sigma$ são elementos arbitrários do reticulado $\mathscr{L}(G), G$ não admite duas topologias de grupo $T_{1}$-independentes.

Os grupos abelianos sem subconjuntos não triviais incondicionalmente fechados podem ser caracterizados em termos da definição anterior; de fato, Tkačenko e Yasčenko em [64] provam que qualquer grupo abeliano satisfazendo essa condição é quase livre de torção (q.l.t.) ou é de ordem prima. De acordo com isto, obtém-se que sob MA, todo grupo topológico 
abeliano 2-enumerável de cardinalidade $\mathfrak{c}$ sem subconjuntos não triviais incondicionalmente fechados admite uma topologia de grupo $T_{1}$-independente e assim, uma topologia de van Douwen. 


\section{Referências Bibliográficas}

[1] B. Anderson, A class of topologies with $T_{1}$-complements, Fund. Math. 67 (1970), 267-277.

[2] _ Families of mutually complementary topologies, Proc. Amer. Math. Soc. 29 (1971), $362-368$.

[3] B. Anderson and D. Stewart, $T_{1}$-complements of $T_{1}$-topologies, Proc. Amer. Math. Soc. 23 (1969), 77-81.

[4] A. Arhangel'sǩ̌i and M.G. Tkačenko, Topological Groups and Related Structures, Atlantis Studies in Mathematics, vol. 1, Atlantis Press/World Scientific, Paris, 2008.

[5] R. Bagley, On the characterization of the lattice of topologies, J. London Math. Soc. 30 (1955), 247-249.

[6] J. E. Baumgartner, Sacks forcing and the total failure of Martin's Axiom, Topology Appl. 19 (1985), 211-225.

[7] J. E. Baumgartner and R. Laver, Iterated perfect-set forcing, Ann. Math. Logic 17 (1979), $271-288$.

[8] M.G. Bell, Compact c.c.c. non-separable spaces of small weight, Topology Proc. 5 (1980), $11-25$.

[9] _ On the combinatorial principle $P_{\mathfrak{c}}$, Fund. Math. 114 (1981), no. 2, 149-157.

[10] A.R. Bernstein, A new kind of compactness for topological spaces, Fund. Math. 66 (1970), 181-193.

[11] G. Birkhoff, On the combination of topologies, Fund. Math. 26 (1936), 156-166.

[12] A. Blass, The Rudin-Keisler ordering of P-points, Trans. Amer. Math. Soc. 179 (1973), 145166.

[13] A.R. Blass and S. Shelah, There may be simple $P_{\boldsymbol{\aleph}_{1}}$-points and $P_{\boldsymbol{\aleph}_{2}}$-points and the RudinKeisler ordering may be downward directed, Ann. Math. Logic 33 (1987), 213-243.

[14] G. Cantor, Ein Beitrag zur Mannigfaltigkeitslehre, Journal für die reine und angewandte Mathematik 84 (1878), 242-258. 
[15] I. Castro-Pereira and A.H. Tomita, A countably compact free Abelian group whose size has countable cofinality, Appl. Gen. Topology 5 (2004), no. 1, 97-101.

[16] W.W. Comfort, Topological Groups, Handbook of Set-Theoretic Topology (K. Kunen and J.E. Vaughan, eds.), North Holland, Amsterdam, 1984, pp. 1143-1263.

[17] _ Problems on topological groups and other homogeneus spaces, Open problems in Topology (G.M. Mill, J. van e Reed, ed.), North Holland, Amsterdam, 1990, pp. 311-347.

[18] W.W. Comfort, K.H. Hofmann, and D. Remus, Topological Groups and Semigroups, Recent Progress in General Topology (M. Hušek and J. van Mill, eds.), Elsevier Sciense Publishers B.V., Amsterdam, 1992, pp. 57-144.

[19] W.W. Comfort and S. Negrepontis, The Theory of Ultrafilters, Grudlehren der Matematischen, no. 211, Springer-Verlag, Berlin, 1974.

[20] W.W. Comfort and D. Remus, Imposing pseudocompact group topologies on Abelian groups, Fund. Math. 142 (1993), 221-240.

[21] W.W. Comfort and K.A. Ross, Pseudocompactness and uniform continuity in topological groups, Pacific J. Math. 16 (1966), no. 3, 483-496.

[22] D. Dikranjan and D. Shakhmatov, Algebraic structure of the pseudocompact groups, Mem. Amer. Math. Soc., vol. 133/633, 1998, pp. viii+83.

[23]__ Forcing hereditarily separable compact-like group topologies on Abelian groups, Topology Appl. 151 (2005), 2-54.

[24] D. Dikranjan and M.G. Tkačenko, Algebraic structure of small countably Abelian groups, Forum Math. 15 (2003), 811-837.

[25] D. Dikranjan, M.G. Tkačenko, and I. Yasčenko, On transversal group topologies, Topology Appl. 153 (2005), 786-817.

[26] E.K. van Douwen, The product of two countably compact topological groups, Trans. Amer. Math. Soc. 262 (1980), 417-427.

[27] _ The weight of a pseudocompact (homogeneus) space whose cardinality has countable cofinality, Proc. Amer. Math. Soc. 80 (1980), no. 4, 678-682.

[28] R. Engelking, Cartesian products and dyadic spaces, Fund. Math. 57 (1965), 287-304.

[29] _ General Topology, Heldermann Verlag, Berlin, 1989.

[30] I. Farah and S. Todorčevich, Some Applications of the Method of Forcing, Yenisei, Russia, 1995.

[31] V. V. Fedorčuk, A compact space having the cardinality of the continuum with no convergent sequences, Math. Proc. Camb. Phil. Soc. 81 (1977), 177-181.

[32] Z. Frolík, The topological product of two pseudocompact spaces, Czechoslovak Math. J. 85 (1960), no. 10, 319-338.

[33] L. Fuchs, Infinite Abelian Groups, vol. I, Academic Press, New York-London, 1970. 
[34] S. Garcia-Ferreira, Various orderings on the space of ultrafilters, Ph.D. thesis, Wesleyan University, 1989.

[35] _ Three ordering on $\beta(\omega) \backslash \omega$, Topology Appl. 50 (1993), 199-216.

[36] J. Ginsburg and V. Saks, Some applications of ultrafilters in topology, Pacific J. Math. 57 (1975), 403-418.

[37] M. Goldstern and H. Judah, The Incompleteness phenomenon: a new course in mathematical logic, A.K. Peters, Wellesley, Mass, 1995.

[38] A. Hajnal and I. Juhász, Remarks on the cardinality of compact spaces and their Lindelöf subespaces, Proc. Amer. Math. Soc. 59 (1976), 146-148.

[39] __ A separable normal topological group need not be Lindelöf, Gen. Topology Appl. 6 (1976), no. 2, 199-205.

[40] K.P. Hart and J. van Mill, A countably compact topological group $H$ such that $H \times H$ is not countably compact, Trans. Amer. Math. Soc. 323 (1991), 811-821.

[41] F. Hausdorff, Grundzüge einer Theorie der geordneten Mengen, Matematishe Annalen 65 (1908), 435-505.

[42] R. Hodel, On the weight of a topological space, Proc. Amer. Math. Soc. 43 (1974), 470-474.

[43] _ Cardinal functions I, Handbook of Set-Theoretic Topology, Elsevier Sciense Publishers B.V., 1984, pp. 1-61.

[44] T. Jech, Set Theory, Springer-Verlag, Berlin, 2003.

[45] I. Juhász, Cardinal functions in topology, Math. Centre Tracts, vol. 34, Math. Centrum, Amsterdam, 1971.

[46] P.B. Koszmider, A.H. Tomita, and S. Watson, Forcing countably compact groups topologies on a larger free Abelian group, Topology Proc. 25 (2000), 563-574.

[47] K. Kunen, Set theory: An introduction to Independence Proofs, Elsevier B.V., Amsterdam, 1980.

[48] K. Kunen and F. Tall, Between Martin's Axiom and Souslin's hypothesis, Fund. Math. 102 (1979), 173-181.

[49] R. Laver, Products of infinitely many perfect trees, J. London Math. Soc. 29 (1984), no. 3, 385-396.

[50] R.E. Madariaga-Garcia and A.H. Tomita, Countably compact topological group topologies on a free Abelian groups from selective ultrafilters, Topology Appl. 154 (2007), no. 7, 14701480.

[51] V.I. Malykhin and L.B. Shapiro, Pseudocompact groups without converging sequences, Mat. Zametki 37 (1985), no. 1, 59-66.

[52] A. Mukherjea and N.A. Tserpes, A note on countably compact semigroups, J. Austral. Math. Soc. 13 (1972), 180-184. 
[53] D. Robbie and S. Svetlichny, An answer to A. D. Wallace's question about countably compact cancellative semigroups, Proc. Amer. Math. Soc. 124 (1996), no. 1, 325-330.

[54] A. van Rooji, The lattice of all topologies is complemented, Canad. J. Math. 20 (1968), 805807.

[55] V. Saks, Products of countably compact spaces, Topology Proc. 4 (1979), 553-575.

[56] V. Saks and R. M. Stephenson, Products of m-compact spaces, Proc. Amer. Math. Soc. 28 (1971), 279-288.

[57] S. Shelah, Proper forcing, Lecture Notes in Mathematics, vol. 940, Springer-Verlag, BerlinNew York, 1982.

[58] S.M. Sirota, The product of topological groups and extremal disconnectedness, Math. USSR Sbornik 8 (1969), no. 2, 169-180.

[59] A. Steiner, Complementation in the lattice of $T_{1}$-topologies, Proc. Amer. Math. Soc. 17 (1966), 884-886.

[60] A. Steiner and E. Steiner, A $T_{1}$-complement for the reals, Proc. Amer. Math. Soc. 19 (1968), 177-179.

[61] E. Steiner, Topologies with $T_{1}$-complements, Fund. Math. 61 (1967), 23-28.

[62] M.G. Tkačenko, Countably compact and pseudocompact topologies on free Abelian groups, Iz. VUZ. 34 (1990), no. 5, 68-75.

[63] _ Introduction to topological groups, Topology Appl. 86 (1998), 179-231.

[64] M.G. Tkačenko and I. Yasčenko, Independent group topologies on Abelian groups, Topology Appl. 122 (2002), no. 1-2, 425-451.

[65] A.H. Tomita, The Wallace problem: a counterexample from $M A_{\text {countable }}$ and p-compactness, Canad. Math. Bull. 39 (1996), no. 4, 486-498.

[66] - The existence of initially $\omega_{1}$ compact group topologies on free Abelian groups is independent of ZFC, Comment. Math. Univ. Carolin. 39 (1998), no. 2, 401-413.

[67] _ Two countably compact topological groups: One of size $\boldsymbol{\aleph}_{\omega}$ and the other of weight $\boldsymbol{\aleph}_{\omega}$ without non-trivial convergent sequences, Proc. Amer. Math. Soc. 131 (2003), no. 8, $2617-2622$.

[68] A solution to Comfort's question on the countable compactness of powers of a topological groups, Fund. Math. 186 (2005), 1-24.

[69] A.H. Tomita and S. Watson, Ultraproducts, p-limits and antichains on the Comfort group order, Topology Appl. 143 (2004), 147-157.

[70] J.E. Vaughan, Countable compact and sequentially compact spaces, Handbook of SetTheoretic Topology (K. Kunen and J.E. Vaughan, eds.), North Holland, Amsterdam, 1984, pp. 569-602.

[71] A.D. Wallace, The structure of topological semigroups, Bull. Amer. Math. Soc. 61 (1955), 95-112. 


\section{Índice Remissivo}

$\perp, 10$

$\Vdash, 11$

$\mathbb{I}^{*}, 12$

$\leq_{\mathrm{RK}}, 62$

$\leq_{\mathrm{C}}, 65$

$\leq_{\mathrm{CG}}, 65$

$[f]_{p}, 67$

$\left([\mathrm{c}]^{<\omega}\right)^{\omega} / p, 67$

$\|\cdot\|, 81$

$\|\cdot\|_{1}, 81$

||$|\cdot|||, 84$

$\|\cdot\|_{\mathrm{T}}, 87$

๑, 104

$\mathrm{A}(X), 37$

anticadeia, 10

árvore, 13

perfeita, 13

Baumgartner J., 16

$\beta \omega, 62$

caráter, 132

cardinal

fracamente inacessível, 7

inacessível, 7

limite forte, 7

c.c.c.

espaço, 16

ordem, 12

$\mathrm{CH}, 7$

Čech-Pospiśsil

teorema de, 22

Čech-Stone

compactificação de, 62

clossing-off, 104
Cohen P., 9

forcing de, 13

real de, 13

Comfort W.W., 27, 97

ordem de, 65

conjunto

centrado, 17

co-denso, 19

definível, 9

denso

em um espaço topológico, 19

em uma ordem parcial, 10

denso em si mesmo, 19

disperso, 19

$G_{\delta}$-denso, 19

hereditariamente finalmente denso, 28

incondicionalmente fechado, 128

raro, 19

$\operatorname{def}(M), 9$

$\Delta$-sistema, 18

lema do, 18

Douwen E. van, 27, 97

Easton

forcing de, 14, 15, 96, 106

função de, 14

espaço

de Tychonoff, 19

de Baire, 19

diádico, 19

enumeravelmente compacto, 20

homogêneo, 24

normal, 19

p-compacto, 64 
pseudocompacto, 20, 99

regular, 19

extensão genérica, 11

$\vec{F}, 67$

$\mathrm{F}(X), 37$

família de funções

produto diagonal de uma, 19

separa pontos, 19

filtro, 10, 61

base de, 61

genérico, 10

livre, 62

principal, 62

$\operatorname{Fn}(A, B), 5$

forcing, 9

condições de um, 10

iteração de, 15

princípio de, 10

produto de, 14

relação de, 11

$\hat{G}, 132$

Garcia-Ferreira, 65

$\mathrm{GCH}, 7$

grupo

booleano, 29

conjunto livre de geradores de um, 37

de torção, 23

de van Douwen, 28

divisível, 23

livre de torção, 23

ordem de um, 23

quase livre de torção, 130

topológico, 24

livre, 36

quociente, 26

subgrupo de, 25

topologia de, 24

Hewitt-Marczewski-Pondiczery

teorema de, 22

incomparáveis, 10

incompatíveis, 10

Ivanovskiǐ-Kuz'minov

teorema de, 98

König

lema de, 7
К-c.c., 12

Kunen K.

axioma de, 122

$\mathbb{L}, 9$

$\mathscr{L}(G), 126$

$\lambda$-centrada, 17

$\lambda$-fechada, 13

$\lambda_{a}, 23$

Laver R., 16, 95

$M^{\mathbb{P}}, 11$

$M[\mathbb{G}], 11$

MA, 16

falha de, 94

no sentido de Baumgartner, 94

versão topológica, 17

$\mathrm{MA}(\kappa), 16$

$\mathrm{MA}_{\text {enumerável }}, 17$

$\mathrm{MA}_{\text {o-centrada, }}, 17$

modelo, 8

Numakura K.

teorema de, 120

$\mathbb{O N}, 6$

$p_{A}, 19$

$p$-equivalência, 67

p-limite, 63

$\mathbb{P}$-nome, 11

interpretação de um, 11

$P$-ponto, 63

palavra, 37

$\pi_{s}, 19$

Pontryagin L.S., 37

grupo dual de, 132

$\mathbb{R} / \mathbb{Z}$, veja $\mathbb{T}$

$\mathrm{Q}_{a}, 23$

Rudin-Keisler

ordem de, 62

S, 13

$\mathbb{S}_{\alpha}, 16$

$\mathbb{S}_{\omega}^{\kappa}, 16$

Sacks

forcing de, 13

iterado, 16, 95

side-by-side, 15, 95, 96

real de, 13, 16 
Saks V., 65

$\mathrm{SCH}, 104$

semigrupo, 23

cancelativo, 119

de Wallace, 121

topológico, 24

sentença, 8

independente de uma teoria, 8

$\sigma$-centrada, 17

$\mathbb{T}, 23-26,38$

$\mathbb{T}^{\mathrm{K}}, 25$

$\mathscr{T}(X), 125$

$\mathscr{T}_{1}(X), 125$

$\tau_{\mathbb{G}}, 11$

$\tau_{\text {Sor }}, 25$

teoria, 8

completa, 8

consistente, 8

Tkačenko M.G., 28, 35, 39

grupo de, 121

topologias

complementares, 125

$T_{1}$-complementares, 125

$T_{1}$-independentes, 127

transversais, 127

$\operatorname{tor}(G), 23$

torção, 23

ultrafiltro, 62

seletivo, 62,63

ultraproduto, 67

$\mathbb{V}, 8$

$\mathbb{V}^{\mathbb{P}}, 11$

Wallace A.D., 121

problema de, 121

semigrupo de, 121 